\title{
Albert RAUGI \\ Fonctions harmoniques sur les groupes localement compacts à base dénombrable
}

\author{
Mémoires de la S. M. F., tome 54 (1977), p. 5-118 \\ <http://www.numdam.org/item?id=MSMF_1977_54_5_0>
}

CC Mémoires de la S. M. F., 1977, tous droits réservés.

L'accès aux archives de la revue « Mémoires de la S. M. F. » (http://smf. emath.fr/Publications/Memoires/Presentation.html) implique l'accord avec les conditions générales d'utilisation (http://www.numdam.org/conditions). Toute utilisation commerciale ou impression systématique est constitutive d'une infraction pénale. Toute copie ou impression de ce fichier doit contenir la présente mention de copyright.

\section{Numdam}




\title{
FONCTIONS HARMONIQUES SUR LES GROUPES
}

\section{LOCALEMENT COMPACTS A BASE DENOMBRABLE}

\author{
par Albert RAUGI
}

\section{INTRODUCTION.}

Soient $G$ un groupe localement compact à base dénombrable (L.C.D.) et $\mu$ une mesure de probabilité sur G (mesure positive de masse 1 sur la tribu barélienne de G). Nous appelons fonction $\mu$-harmonique bornée toute fonction borélienne bornée vérifiant l'équation

$$
f(g)=\int_{G} f(g h) \mu(d h) \quad(g \in G)
$$

Celles-ci ont été étudiées de façon appronfondie par Furstenberg [8] et Azencott [1] :

En généralisant une construction probabiliste due à Furstenberg. Azencott obtient une représentation intégrale des fonctions $\mu$-harmoniques bornées. Soient $E_{\mu}$ l'espace de Banach des fonctions $\mu$-harmoniques bornées (muni de la norme de la convergence uniforme) et $H_{\mu}$ le sous-espace formé des éléments de $E_{\mu}$ uniformément continus à gauche (u.c.g.). Muni du produit

$$
f \times f^{\prime}(g)=\lim _{n} \int_{G} f(g h) f^{\prime}(g h) \mu^{n}(d h) \quad\left(f, f^{\prime} \in H_{\mu}\right),
$$

où $\mu^{n}$ désigne le produit de convolution de $n$ mesures égales à $\mu$, $H_{\mu}$ est une $C^{*}$-algèbre. Le spectre $\Pi_{\mu}$ de cette $C^{*}$-algètre est appelé l'espace de Poisson de $\mu$; c'est un espace compact sur lequel $G$ opère continûment. Lorsque $\mu$ est étalée sur $G$, (i.e. qu'il existe un entier $p$ tel que $\mu^{P}$ ne soit pas singulière par rapport à une mesure de Haar sur G), il existe ure mesure de probabilité $v$ sur $\Pi_{\mu}$ (noyau de Poisson de $\mu$ ) et une mesure quasi-invariante $\varepsilon$ sur $\Pi_{\mu}$ telles que l'application $f \longmapsto f * v: g \longmapsto \int_{\Pi_{\mu}} f(g \cdot x) v(d x),(g \in G)$, soit une isométrie de $L^{\infty}\left(\pi_{\mu}, \varepsilon\right)$ sur $E_{\mu}$ envoyant $C\left(\pi_{\mu}\right)^{\mu}$ (espace des fonctions continues sur $\Pi_{\mu}$ ) sur $H_{\mu}$. L'espace $\Pi_{\mu}$ et le noyau $\nu$ sont déterminés à isomorphisme près par cette dernière propriété.

Lorsque $\mu$ est étalée l'étude du cas des groupes L.C.D. G tels que $G / G_{0}$ soit compact ( $G_{0}$ est la composante connexe de l'unité dans $\left.G\right)$ se ramène à celle des groupes de Lie ayant un nombre fini de composantes connexes.

Nous disons ([1]) qu'un groupe L.C.D. G est de type ( $T$ ) si pour toute mesure de probabilité étalée $\mu$ sur $G$, l'espace de Poisson $\pi_{\mu}$ est homogène. Parmi ces groupes on trouve: les groupes de Lie connexes moyennables $G$ tels que les va- 
leurs propres des éléments de Ad R, image du radical $R$ de $G_{0}$ par la représentation adjointe de $G$, soient de modules égaux à 1 ([1]); les groupes de Lie connexes semi-simples de centre fini ([8]) et les groupes de Lie connexes qui sont "localement produit direct de ces groupes" ([4]). Pour les groupes de type (T) on connaît explicitement l'espace de Poisson $\pi_{\mu}$ (voir [1]).

Dans cet article nous étudions essentiellement le cas où $G$ est un groupe L.C.D. tel que $G_{0} \backslash^{G}$ soit compact et $\mu$ est étalée et possède un moment d'ordre 1 . L'hypothèse " ${ }^{\prime}{ }^{G}$ de centre fini" qui apparaissait dans [21] a pu être supprimée grâce à un résultat de Rosenberg (voir [24]). Dans cette situation, nous montrons, qu'en général, l'espace de Poisson n'est pas "un bon espace de représentation". Nous établissons une nouvelle représentation intégrale des fonctions $\mu$-harmoniques bornées à l'aide d'un espace homogène $\Lambda_{\mu}$ de $G$; dans le cas où l'espace de Poisson $\Pi_{\mu}$ est homogène, les espaces $\Lambda_{\mu}$ et $\Pi_{\mu}$ coinncident ; dans les autres cas $\pi_{\mu}$ est une compactification non métrisable de $\Lambda_{\mu}$.

Donnons une description rapide de l'espace $\Lambda_{\mu}$, en nous limitant, pour simplifier, au cas où $G$ est un groupe de Lie connexe :

Désignons par ef l'algèbre de Lie de $G$; par $R$ le radical de ef et par

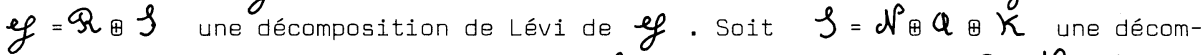
position d'Iwasawa de l'algèbre de Lévi $f$ de $y$. Posons $D=R \oplus \mathbb{P} Q$ et désignons par $D$ et $K$ les sous-groupes de Lie connexes de $G$ ayant respectivement $D$ et $K$ pour algèbres de Lie. Nous obtenons la décomposition $G=D K$, avec $D \cap K$ central et discret.

Soit $B(G)$ la frontière maximale gauche de $G([1])$; nous savons que $K$ est transitif sur $B(G)$. Nous appelons A-cocycle ([10]), K-invariant sur $B(G)$, toute application continue $\rho$ de $B(G) \times G$ dans $\mathbb{R}$ telle que

i) $\rho\left(u, g_{1} g_{2}\right)=p\left(u, g_{1}\right)+p\left(u, g_{1}, g_{2}\right) \quad\left(u \in B(G) ; g_{1}, g_{2} \in G\right)$

ii) $\rho(u, k)=0 \quad(u \in B(G) ; k \in K)$.

Soit $\mu$ le. nilradical de $\mathbb{R}$ et $\mathcal{Q}$ une sous algèbre de Cartan du centralisateur de $\mathcal{S}$ dans $\boldsymbol{R}$. Nous avons alors

$$
\mathscr{R}=\mathscr{H}+\boldsymbol{S} \text { (somme non nécessairement directe). }
$$

$S \oplus Q$ est une sous-algèbre nilpotente de $\mathcal{f}$ qui est le produit direct des sousalgèbres $Q$ et $\boldsymbol{Q}$. Désignons par $\theta$, l'ensemble des poids de la représentation adjointe de $\mathscr{P} \oplus \mathbb{Q}$ dans la complexifiée $y^{\mathbb{C}}$ de $\boldsymbol{y}$. Notons $M, P$, $A$ et $N$ les sous-groupes de Lie connexes de $G$ ayant respectivement $\mu_{b}, \mathcal{S}, Q$ et $\mathcal{N}$ pour algèbres de Lie; nous avons la décomposition $G=M N P A K$. Pour tout élément $\theta$ de $\theta$, soit $\phi_{\theta}$ le poids de la représentation adjointe de $P$ A dans $y^{\mathbb{C}}$ dont la différentielle en $e$ est $\theta$ et posons

$$
\rho_{\theta}\left(u_{0} \cdot k, g\right)=\log \left|\phi_{\theta}(x)\right| \text {, }
$$


où $U_{0}$ désigne l'unique élément de $B(G)$ stable par $D$ et où $k g$ s'écrit $m \times k$ avec $m \in M N, x \in P A$ et $k \in K$.

Cette définition est en fait sans ambiguité et fournit une famille d'A-cocycles K-invariants $\left\{\rho_{\theta}, \theta \in \theta\right\}$, étudiée au $\delta 7$.

Soit $\mu$ une mesure de probabilité étalée et possèdant un moment d'ordre 1 sur $G$. Nous savons ([8]) qu'il existe sur $B(G)$ une unique mesure de probabilité $\nu$, $\mu$-invariante (i.e. telle que $\nu * \mu=\nu$ ) ; définissons

$$
\begin{aligned}
& \theta_{-}=\left\{\theta \in \theta: \int_{G} \int_{B(G)} \rho_{\theta}(u, g) \nu(d u) \mu(d g)<0\right\} \\
& \theta_{+}=\left\{\theta \in \theta: \int_{G} \int_{B(G)} \rho_{\theta}(u, g) v(d u) \mu(d g) \geqslant 0\right\} .
\end{aligned}
$$

Pour tout élément $\theta$ de $\theta$, soit

$$
y_{\theta}=\left\{x \in \mathscr{y}^{\mathbb{C}}: \exists n \in N^{*} /(\operatorname{adH}-\theta(H) I)^{n}(X)=0, \forall H \in \mathbb{P}_{\mathbb{Q}} \mathbb{Q}\right\} ;
$$

nous avons $([25]) \quad y^{\mathbb{C}}=\underset{\theta \in \theta}{\mathbb{P}} \mathscr{f}_{\theta}$. Posons

$$
y_{-}^{\mathbb{C}}=\underset{\theta \in \theta_{-}}{\mathbb{B} y_{\theta}}, y_{+}^{\mathbb{C}}=\underset{\theta \in \theta_{+}}{\boxplus} y_{\theta}
$$

Alors $y_{-}^{\mathbb{C}}$ et $y_{+}^{\mathbb{C}}$ sont deux sous-algèbres de $\boldsymbol{y}^{\mathbb{C}}$ qui sont les complexifiées de deux sous-algèbres $y_{-}$et $y_{+}$de $y$ telles que $y=y_{-} \boxplus y_{+}$; et si on désigne par $G_{-}$et $G_{+}$les sous-groupes de Lie connexes de $G$ ayant respectivement $\mathcal{f}_{\text {- et }}$ $y_{+}$pour algèbre de Lie, $G_{-}$et $G_{+}$. sont deux sous-groupes fermés de $G$, $G_{-} \cap G_{+}=\{e\}$ et $G=G_{-} G_{+}$modulo une quelconque mesure de Haar de $G$.

On obtient alors une représentation intégrale des fonctions $\mu$-harmoniques bornées à l'aide d'un revêtement à fibre dénombrable (explicitement décrit), $\Lambda_{\mu}$, de $G / G_{+}$ (théorème $\left(8.4\right.$ )). Dans le cas où $R^{G}$ est de centre fini, ce revêtement est fini.

La sous-algèbre $y_{-}$est contenue dans $D$; posons $D_{+}=D_{\cap} \mathscr{y}_{+}$et désignons par $D_{+}$le sous-groupe de Lie connexe de $D$ ayant $D_{+}$pour algèbre de Lie. Soit $E_{+}=D_{+} K$; $E_{+}$est une sous-variété de $G$ et l'application qui au couple $(h, g)$ associe le produit $h g$ est un isomorphisme de variétés analytiques de $G_{-} \times E_{+}$ sur $G$. Le procédé utilisé pour obtenir la représentation des fonctions $\mu$-harmoniques bornées à l'aide d'un revêtement $\Lambda_{\mu}$ de $G / G_{+}$, est alors basé sur les deux faits suivants :

i) Désignons par $\left(\Omega, \mathscr{F},\left(x_{n}\right)_{n \in \mathbb{N}},\left(\mathbb{P}_{x}\right)_{x \in G}\right)$ la marche aléatoire droite de loi $\mu$ sur $G$. Nous montrons ( $\$ 9$ ) que la composante de $\aleph_{n}$ sur $G_{-}$, dans la décomposition $G=G_{-} E_{+}$, converge $\mathbb{P}_{x}$-p.s. $\forall x \in G$. De plus si on note $Z_{g}$ la limite de la composante de $\mathrm{gX}_{\mathrm{F}_{+}}$sur $G_{-}$; on montre alors que si on identifie $G_{-}$à un 
ouvert de $G / G_{+}$,

$$
z_{g}=g \cdot z_{e}, \mathbb{P}_{x}-\text { p.s. } \forall x \in G .
$$

ii) Soit $H$ un sous-groupe fermé de $G$, nous appelons fonction $\mu$-harmonique bornée sur $H^{G}$, toute fonction $f$ sur $H^{G}$ telle que fop soit $\mu$-harmonique bornée, où $p$ désigne l'application naturelle de $G$ sur $H^{G}$. Nous étendons à cette situation un résultat de [1] sur les périodes des fonctions $\mu$-harmoniques bornées (théorème ( 6.8 )). Nous montrons ( $\$ 10)$ que les fonctions $\mu$-harmoniques bornées sur $G_{-} \backslash^{G}$ sont les composées par l'application naturelle de ${ }{ }^{G}$ sur $D^{G}$ des fonctions $\mu$-harmoniques bornées sur $D^{G}$. On montre que ces fonctions prennent un nombre dénombrable de valeurs; ce nombre est fini si $R^{G}$ est de centre fini.

Ensuite, nous comparons les espaces $\Lambda_{\mu}$ et $\pi_{\mu}$ ( $\$ 11$ ); nous étudions les groupes de type $(T)$ ( $\$ 12$ ) et nous passons du cas des groupes de Lie au cas des groupes L.C.D. ( $\$ 13)$.

Ce travail fait partie d'une thèse de doctorat d'état dirigée par R. Azencott. Je tiens à remercier vivement $R$. Azencott pour son aide et ses encouragements constants, ainsi que $Y$. Guivarc'h avec qui j'ai eu des discussions fructueuses. 


\section{PRELIMINAIRES}

1.0.- $\mathscr{L}$ étant une algèbre de Lie réelle, nous notons $\mathscr{L}^{\mathbb{C}}$ sa complexifiée et $\mathscr{L}^{*}$ l'espace des formes linéaires sur $\mathscr{L}$ à valeurs complexes.

Soit $\rho$ une représentation d'une algèbre de Lie réelle $\mathscr{L}$ (resp. d'un groupe de Lie L ) dans un espace vectoriel (réel ou complexe) $V$ et $\gamma$ un élément de $L^{*}$ (resp. de l'ensemble des homomorphismes de. $G$ dans $\mathbb{C}$ ). Nous disons que $\gamma$ est un poids de $\rho$ s'il existe un sous-espace $W$ de $V$ stable par $\rho$ et un vecteur $\checkmark$ de $V-W$ tels que $\forall x \in \mathcal{L}$ (resp. $L$ ), $\rho(x)(v)=\gamma(x) \cdot v$ modulo $W$;

l'élément $\vee$ de $V-W$ est alors appelé un vecteur presque propre associé au poids $\gamma$.

D'autre part, si $\gamma \in \mathscr{L}^{*}$, nous désignons par $V_{\gamma}$ le sous-espace de $V$ formé des éléments qui pour chaque $x$ de $\mathscr{L}$ sont annulés par au moins une puissance de $\rho(x)-\gamma(x) I$ ( I désigne l'identité de $V$ ). Dans le cas où l'algèbre de Lie $\mathscr{L}$ est nilpotente et $V$ est un espace vectoriel sur le corps des complexes, nous savons que (voir [25]) : les espaces $V_{\gamma}$ sont stables par $\rho$; si $V_{\gamma} \neq(0)$, $\gamma$ est le seul poids de $\rho$ dans $V_{\gamma}$ et $V$ est la somme directe des sous-espaces $V_{\gamma}$.

1.1.- Soit $G$ un groupe de Lie ayant un nombre fini de composantes connexes. On note $G_{0}$ la composante connexe de l'unité dans $G$ et $R$ le radical de $G_{0}$ (i. e. le plus grand sous-groupe résoluble distingué connexe de $G_{0}$; $R$ est un sous-groupe fermé de $G_{0}$ et donc de $G$ J. On désigne par $y$ et $R$ les algèbres de Lie respectives de $G$ et $R$.

Soit $\boldsymbol{J}=\boldsymbol{R} \oplus \mathcal{S}$ une décomposition de $\boldsymbol{y}$ comme somme directe de son radical $\boldsymbol{R}$ et d'une sous-algèbre semi-simple $\mathcal{S}$ (décomposition de Lévi); on sait ([5], théorème de Lévi-Malcev) que deux telles décompositions se déduisent l'une de l'autre par un automorphisme de $\mathcal{H}$ de la forme Exp ad $X$, où $X$ appartient à l'idéal nilpotent $[y, R]$.

1.2. - Nous allons introduire une décomposition d'Iwasawa de la sous-algèbre de Lévi $\mathcal{I}$. La description donnée ci-dessous résume des résultats classiques (voir, par exemple, [13]).

Soit $\mathcal{S}=\boldsymbol{K} \oplus \boldsymbol{\gamma}$ une décomposition de Cartan de $\mathcal{S}$ et $Q$ un sous-espace abélien maximal de $\hat{\gamma}$.

Considérons la représentation adjointe de $a$ dans $\mathcal{S}$; soit $\sum$ l'ensemble des poids de cette représentation. Tout élément de $\Sigma$ est à valeurs réelles et nous avons : 


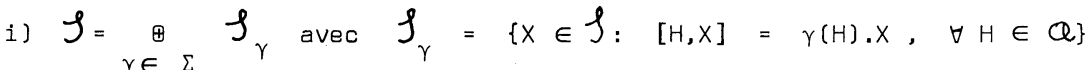

ii) $J_{0}=\varphi \boxplus m$ où $m$ est le centralisateur de $Q$ dans $K$

iii) $\left[\rho_{\gamma}, \mathcal{\rho}_{\gamma^{\prime}}\right] \subset \mathcal{\rho}_{\gamma+\gamma^{\prime}}, \forall \gamma$ et $\gamma^{\prime} \in \Sigma$

iv) Si $\gamma \in \Gamma, \quad(-\gamma) \in \Sigma$

Soit $G$ ' l'ensemble des éléments de $Q$ où aucun élément de $\Sigma-\{0\}$ ne s'annule. Les composantes connexes de $Q$, (en nombre fini) sont les chambres de Weyl. Choisissons une chambre de Weyl $W$. Soit $\Sigma_{1}$ ( resp. $\Sigma_{2}$ ) l'ensemble des éléments de $\Sigma$ négatifs ( resp. positifs ) sur $W$.

Posons $\mathcal{N}=\underset{\gamma \in \Sigma}{\mathbb{P}} \mathcal{J}_{\gamma}$ et $\tilde{\mathcal{N}}=\underset{\gamma \in \Sigma_{2}}{\oplus} \mathcal{J}_{\gamma}$. $\mathcal{P}$ et $\tilde{\mathcal{P}}$ sont des sous-algèbres 'nilpotentes de ${ }^{\gamma} \mathcal{\rho} \in \Sigma_{1} \mathcal{P} \oplus Q$ est une sous-algèbre résoluble de $\mathcal{S}$. On obtient une décomposition d'Iwasawa, soit $\mathcal{S}=\mathcal{P} \oplus Q \oplus K$. Une telle décomposition dépend des choix de la décomposition de Cartan $\mathcal{S} K \boxplus \boldsymbol{\gamma}$, du sous-espace abélien maximal $Q$ de $\boldsymbol{\gamma}$ et de la chambre de Weyl $W$. Cependant on sait que deux telles décompositions se déduisent par un automorphisme intérieur de $\mathcal{S}$.

1.3.- Posons $D=Q \cap \mathcal{P} \oplus Q$. Nous obtenons la décomposition

$$
y=D \oplus K \text {. }
$$

où $D$ est une sous-algèbre résoluble et $K$ une sous-algèbre compacte de $\mathcal{J}_{\mathcal{f}}$. La décomposition ainsi obtenue dépend des choix de la sous-algebre de Lévi $\mathcal{S}$ et de sa décomposition d'Iwasawa. Deux telles décompositions se déduisent par un automorphisme intérieur de $\mathcal{g}$.

1.4.- Désignons par $S$ le sous-groupe de Lie connexe de $G_{0}$ ayant $\mathcal{f}$ pour algèbre de Lie. Nous avons $G_{0}=R S$ et $R \cap S$ est un sous-groupe de $G_{0}$, discret, distingué, donc central. On notera que $S$ n'est pas nécessairement fermé dans $G_{0}$.

Soient $N, \tilde{N}$, A et $K$ les sous-groupes de Lie connexes de $S$ ayant respectivement $\mathcal{N}, \tilde{\mathcal{P}}, Q$ et $K$ pour algèbre de Lie. Les sous-groupes $N, \tilde{N}, A$ et $K$ sont fermés dans $S$. L'application

$$
(n, a, k) \longmapsto n \text { a } k(n \in N, a \in A, k \in k) \text {, }
$$

est un isomorphisme de variétés analytiques de $N \times A \times K$ sur $S$ ( voir [13]); les groupes $A, N$ et $\tilde{N}$ sont simplement connexes, respectivement abélien et nilpotents; $K$ contient le centre $Z(S)$ de $S$ et est compact si et seulement si $S$ est de centre fini. En outre $K$ est son propre normalisateur dans $S$.

Soit $\Gamma$ le centralisateur de $A$ dans $K$ ( $\Gamma$ contient $Z(S)$ ) ; alors $\Gamma$ et A normalisent $N$, ainsi que $\tilde{N}$. De plus $N$ A $\Gamma$ est le normalisateur de $N$ 
dans $S$.

1.5. Lemme. - Soit D le sous-groupe de Lie connexe de $G_{0}$ ayant $D$ pour algèbre de Lie. $D$ est un sous-groupe de Lie résoluble connexe fermé de $G$. $L$ 'application $(\mathrm{r}, \mathrm{n}, \mathrm{a}) \longmapsto \mathrm{r} n$ a est un isomorphisme de variétés analytiques de $\mathrm{R} \times \mathrm{N} \times \mathrm{A}$ sur $\mathrm{D}$; en particulier les sous-groupes $\mathrm{N}$ et $\mathrm{A}$ sont fermés dans $G$.

Preuve. - Soit $\xi$ l'application naturelle de $G$ sur $R^{I^{G}} \cdot \xi\left(G_{0}\right)=\xi(N) \xi(A) \xi(k)$ est une décomposition d'Iwasawa du groupe de Lie semi-simple $\xi_{0}\left(G_{0}\right)$; nous savons que $\xi(N) \xi(A)=\xi(D)$ est fermé dans $\xi\left(G_{0}\right)$; par suite $D$ est fermé dans $\dot{G}_{0}$.

Il est évident que l'on a $D=R N A$. Le sous-groupe $R \cap N A$ est contenu dans le sous-groupe $R \cap S$. Or $R \cap S$ est contenu dans le centre $Z(S)$ de $S$ et donc dans $\Gamma$. Comme $N A \cap K=\{e\}$, il s'ensuit que $R \cap N A=\{e\}$. Tout élément $d$ de $D$ s'écrit alors de façon unique sous la forme $d=r n$ a avec $r \in R, n \in N$ et $a \in A$. Autrement dit l'application analytique $n: R \times N \times A \longrightarrow D$

$$
(\mathrm{r}, \mathrm{n}, \mathrm{a}) \longmapsto \mathrm{rna}
$$

est bijective.

Comme $D=\mathscr{R} \oplus \mathcal{N} \oplus Q$, on sait que $n$ est un isomorphisme de variétés analytiques d'un ouvert $U \times V \times W$ de $R \times N \times A$ contenant ( $e$, $e$, $e$ ) sur un ouvert $X$ de $D$ contenant $e$. Soit alors $d=r n a \in D$ avec $r \in R, n \in N$ et $a \in A$; la restriction de $n^{-1}$ à. l'ouvert $X^{\prime}=d X$ se décompose en

$$
\begin{aligned}
& x \longrightarrow x \stackrel{n^{-1}}{\longrightarrow} U \times v \times w \longrightarrow R \times N \times A \\
& x \longmapsto d^{-1} \times \quad(u, v, w) \longmapsto\left(r\left(n a u(n a)^{-1}\right) \text {, nava } a^{-1} \text {, aw }\right)
\end{aligned}
$$

et est par suite analytique. L'application $\eta$ est donc un isomorphisme de variétés analytiques. Le lemme $(1.5)$ est prouvé.

1.6. Lemme. - Soit Ady Za représentation adjointe de $G$ dans y . Les sous-

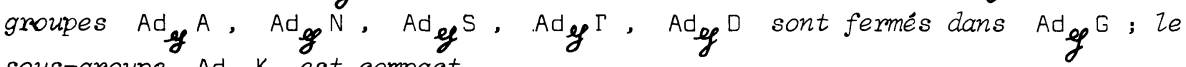
sous-groupe $\mathrm{Ad}_{\boldsymbol{g}} \mathrm{K}$ est compact.

Preuve. - Le radical de $A d g_{0}$ est $A d_{y} y ; A d_{y} G_{0}=A d y y^{R} A y^{S}$ est une décomposition de Lévi de $A d_{y} G_{0}$ et $A d_{y} S=A d g N A d y A$ Ad $y^{K}$ est une décomposition d'Iwasawa de $A_{\text {dg }} S . A_{y} D=A d_{y} R A_{y} N A_{y} A$ est un sous-groupe de Lie connexe résoluble fermé de $A_{g} G_{0}$ ( lemme ( 1.5 ) ). Ady $\Gamma$ est le centralisateur de $A_{y} A$ dans $A d_{y^{K}}$ : en effet, soit $k \in K$ tel que Ad $K$ centralise $\mathrm{Ad}^{A}$; il s'ensuit que $a k a^{-1} k^{-1} \in Z\left(G_{0}\right) \cap S$, pour tout $a \in A$; mais $A$ étant connexe et $Z\left(G_{0}\right) \cap S$ discret, il s'ensuit que a $k a^{-1} k^{-1}=e$, pour tout $a \in A$; autrement dit $k \in \Gamma$. 
Ceci dit $A d y^{S}$ est un sous-groupe de Lie connexe semi-simple du groupe linéaire $G L(y)$; on sait qu'un tel sous-groupe est fermé dans GL(y) et est de centre fini. Il s'ensuit que $A_{d^{\prime}} K$ est un sous-groupe compact maximal de $A^{\prime} g^{S}$ et les

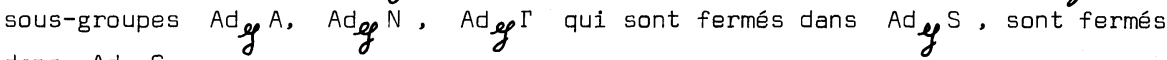
dans $A_{y} G_{0}$.

1.7. Proposition. - Soit $G$ un groupe de Lie ayant un nombre fini de composantes connexes. Désignons par ef l'algèbre de Lie de $\mathrm{G}$; par $\mathbb{R}$ le radical de eg et par $y=R \rightarrow \mathcal{I}$ une décomposition de Lévi de $\mathrm{g}$. Soient $\mathcal{H}$ le nilradical de

$R$ et $\mathcal{S}$ une sous-algèbre de cartan du centralisateur de $\mathcal{I}$ dans $R$. Alors

$Q$ est la somme, non nécessairement directe, de $M$ et de $\mathrm{S}$.

Désignons par P (resp. S) le sous-groupe de Lie connexe de G ayant $\mathrm{Q}$ ( resp. I ) pour algèbre de Lie et par S = N A K une décomposition d'Iwascwa de $S$. Soit $\Gamma^{\prime}$ le sous-groupe de $G$ formé des éléments qui normalisent à la fois $N, A, K$ et $P$ ( et donc aussi $\tilde{N}$ et $\Gamma$ ). $\Gamma^{\prime}$ est un sous-groupe fermé de G rencontrant ses différentes composantes connexes; $I^{\prime}$ intersection de $\Gamma^{\prime}$ avec $G_{0}$ est égale à $P \Gamma$, où $\Gamma$ est le centralisateur de $A$ dans $K$; et $K \Gamma^{\prime}$ est un sous-groupe fermé de $G$ possédant un nombre fini de composantes connexes. KI' possède donc des sous-groupes compacts maximaux deux à deux conjugués; soit $L$ I'un deux et posons $K=K L$; I'image, Ady $K$, de $K$ par $Z a$ représentation adjointe de $G$ est compacte; nous avons alors $G=D K$ et $D \cap K$ est contenu dans $P$.

1.8.- La décomposition $G=D K$ ainsi obtenue dépend des choix de la sous-algètre de Lévi $\mathcal{J}$ de $\mathcal{H}$, de sa décomposition d'Iwasawa et du sous-groupe compact maximal $L$ de $K \Gamma^{\prime}$. Il est facile de voir que deux telles décompositions se déduisent par conjuguaison par un élément de $G_{0}$.

Dans le cas où $G_{0}$ est semi-simple ( on a alors $G_{0}=S$ ), on obtíent la décomposition $G=N A K$ avec $N A \cap K=\{e\}$. Nous dirons que cette décomposition est la décomposition d'Iwasawa de $G$.

Les sections de ( 1.9 ) à ( 1.16 ) sont consacrées à la preuve de la proposition $(1.7$ ). En première lecture on pourra laisser de coté cette preuve qui ne servira pas pour la suite.

Soit $\zeta$ le centralisateur de la sous-algèbre de Lévi $\mathcal{S}$ dans le radical $\mathcal{R}$ de ef (i.e. $\zeta=\{x \in R:[x, \rho]=(0)\})$.

Soit $Q$ une sous-algèbre de Cartan de $\zeta$ ( i.e. une sous-algèbre nilpotente de 6 qui est son propre normalisateur dans $\zeta$, voir [5] ). D'aprèg ( [5], VI, \$4, prop. 19 ) on sait que deux telles sous-algèbres $Q$ et $Q^{\prime}$ de $\zeta$ se déduisent l'une de l'autre par un automorphisme de la forme Exp ad $X$, où $X$ appartient à $[6, \xi]$. 
Soit $\mathcal{H}$ le nilradical de $\boldsymbol{\Omega}$ ( i.e. le plus grand idéal nilpotent de $\boldsymbol{\Omega}$ ). $\boldsymbol{l l}$ contient l'idéal $[\boldsymbol{g}, \boldsymbol{R}$ ] et est formé des éléments $x$ de $\boldsymbol{R}$ tels que ad $X$ soit nilpotent ( $[5], \cdot V, \$ 2$, prop. 4 ).

La première partie de la proposition ( 1.7 ) résulte alors du lemme :

1.9. Lemme. - Le radical $\boldsymbol{h}$ de $y$ est la somme (non nécessairement directe)
des sous-algèbres nilpotentes $\boldsymbol{l}_{\boldsymbol{b}}$ et $\boldsymbol{S}$.

Preuve. - I étant une algèbre de Lie semi-simple, la représentation adjointe de $\mathcal{I}$ dans $\boldsymbol{R}$ est semi-simple. L'idéal $[\boldsymbol{H}, \boldsymbol{R}$ ] de $\boldsymbol{f}$ admet donc, dans $\boldsymbol{R}$. un sous-espace supplémentaire, invariant par cette représentation. Soit $\xi$ ce sous-espace; nous avons :

$$
\boldsymbol{R}=[\boldsymbol{g}, \boldsymbol{R}] \boxplus \xi \text { et }[\mathcal{S}, \xi] \subset[\mathcal{E}, \boldsymbol{R}] \cap \xi=(0) .
$$

Il s'ensuit que

$\boldsymbol{R}=[\boldsymbol{g}, \boldsymbol{R}]+\boldsymbol{b}$ ( somme non nécessairement directe)

où $\zeta$ est le centralisateur de $\mathcal{S}$ dans $\mathscr{R}$.

Considérons la représentation adjointe de la sous-algèbre de Cartan $\varphi$ de $\zeta$ dans $\zeta$ et appelons $\theta$ l'ensemble de ses poids. Nous avons avec les notations de ( 1.0$)$

$$
\zeta_{0}=9, \quad \zeta^{\mathbb{I}}=\underset{\gamma \in \Theta}{\boxplus} \zeta_{\gamma}
$$

Il est facile de voir que pour tout $r \in \theta-\{0\}, \xi_{\gamma} \subset[y, \mathscr{R}]^{\mathbb{C}}$ nous avons alors $\mathscr{R}=[\boldsymbol{g}, \boldsymbol{R}]+\boldsymbol{S}$ et par suite $\boldsymbol{h}=\mu^{r}+\boldsymbol{Q}$.

1.10. Remarque. - Soit gl(V) l'algèbre de Lie de tous les endomorphismes d'un espace vectoriel réel $V$. Supposons que of soit une sous-algèbre de Lie algèbrique de $g l(V)$. On sait ' ( voir [5]) que le radical $\mathcal{Q}$ de $\operatorname{l}$ est une sous-algèbre de Lie résoluble algèbrique de $g l(V)$. Désignons alors par $d l$ l'idéal de $\Omega$ formé, non plus par les éléments $x$ de $\mathcal{R}$ tels que ad $x$ soit nilpotent, mais par les éléments $x$ de $\mathscr{R}$ nilpotents sur $V$. Alors on peut représenter $y$

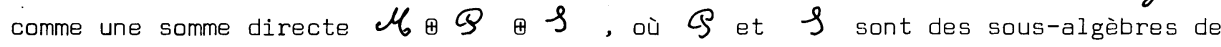
Lie de $\mathcal{g}$ qui possède les propriétés suivantes : $Q$ est abélienne et algèbrique. et ses éléments sont semi-simples ( sur $v$ ) ; le rạdical $R$ de ef est $\boldsymbol{l l}_{\mathbb{P}} \boldsymbol{P}$; \& est semi-simple et ses éléments commutent avec ceux de $Q$. ( voir [5], ch. $V, \$ 4$, prop. 5$\}$.

Dans le cas d'une algèbre de Lie algébrique d'endomorphismes d'un espace vectoriel rée], on peut alors remplacer, dans ce qui suit, la décomposition générale obtenue en ( 1.9 ) par celle obtenue ci-dessus. 
1.11. Remarque. - En passant nous avons obtenu pour $\mathscr{H}$ la décomposition

$$
y=(\mu+\rho) \oplus \mathcal{N} \oplus Q \otimes K \text {. }
$$

Soit $y=\left(\mu b+g^{1}\right) \oplus \mathcal{N}^{1} \boxplus Q^{1} \boxplus K^{1}$ une autre décomposition de $\mathscr{g}$ obtenue par le même procédé.

D'après ce qui a été rappelé antérieurement, il existe :

i) $x_{0} \in M$ tel que $\rho^{1}=\operatorname{Exp}$ ad $x_{0}(\rho)$

ii) $Y_{0} \in\left[\xi^{1}, \xi^{1}\right]$ avec $\xi^{1}=\left\{x \in \mathcal{R}:\left[x, \rho^{1}\right]=(0)\right\}$ tel que $Q^{1}=\left(\operatorname{Exp}\right.$ ad $\left.Y_{0}\right)\left(\operatorname{Exp}\right.$ ad $\left.X_{0}\right)(S)$.

En posant $X_{1}=Y_{0} \times X_{0}=Y_{0}+X_{0}+\frac{1}{2}\left[Y_{0}, X_{0}\right]+\ldots$ (formule de Campbell-Hausdorff $)$, on a alors

$$
S^{1}=\operatorname{Exp} \text { ad } x_{1}(Q) \text { et } S^{1}=\operatorname{Exp} \text { ad } x_{1}(S) \text {. }
$$

Autrement dit deux choix possibles du couple $(\mathcal{S}, S$ ) se déduisent l'un de l'autre par un automorphisme de $\mathcal{g}$ de la forme Exp ad $x$, où $x \in \mathcal{H}$.

Comme deux décompositions d'Iwasawa d'une algèbre de Lie semi-simple se déduisent par un automorphisme intérieur, on en déduit que les deux décompositions considérées de eg se déduisent par un automorphisme intérieur de $\mathcal{f}$.

1.12.- Désignons par $P$ ( resp. M) le sous-groupe de Lie nilpotent connexe de $R$ admettant $Q$ ( resp. $\mu_{b}$ ) pour algèbre de Lie. M est le nilradical de $R$ ( $i . e$. le plus grand sous-groupe distingué nilpotent connexe de $R$ ) et est donc un sous-groupe fermé de $R$ ( et par suite de $G$ ). D'autre part soit $\bar{P}$ l'adhérence de $P$ dans $R$, l'algèbre de Lie $\bar{S}$ du groupe de Lie connexe $\bar{P}$ est nilpotente, contenue dans $\zeta$ et contient $\mathcal{S}$; d'après ( [5], VI, $\$ 4$, prop. 1 ) on sait que $\mathcal{Q}$ est une sous-algèbre nilpotente maximale de $\boldsymbol{b}$; il s'ensuit que $\mathscr{S}=\bar{S}$ et $P=\bar{P}$. $P$ est donc un sous-groupe fermé de $R$ ( et par suite de GJ.

$M$ étant distingué dans $R$, du lemme $(1.9)$ il résulte que l'on a $R=M P$.

Pour achever la preuve de la proposition ( 1.7 ), nous commençons par établir deux lemmes.

1.13. Lemme. - Soit $\mathrm{C}$ le centralisateur de $\mathrm{S}$ dans $\mathrm{R}$. Nous avons

$$
c=\exp _{M}(\mu \cap \xi) P
$$

et $P$ est son propre normalisateur dans $C$. ( En particulier $P$ est un sousgroupe de Cartan de C, voir [5] ).

Preuve: - Métant un groupe de Lie nilpotent, nous avons $M=\exp \mu$. Soit $\mu_{1}=\mu_{6}, \mu_{2}=\left[\mu_{6} \mu_{1}\right], \ldots, \mu_{r}=\left[\mu_{1}, \mu_{r-1}\right], \mu_{r+1}=(0)$, 
la suite centrale descendante de l'idéal nilpotent $\boldsymbol{M b}$ de $\boldsymbol{e}$. Pour $i \in\{1, \ldots, r\}$, désignons par $m^{i}$ un sous-espace supplémentaire de $\mathscr{H}_{i+1}$ dans $\mathscr{H}_{i}$. Nous avons

$$
\mu=m^{1} \oplus m^{2} \oplus \ldots \oplus m^{r} .
$$

Soit $c=(\exp x) p \in C$, avec $X \in \boldsymbol{M}$ et $p \in P$. Comme $P$ est contenu dans

$C$, exp $X$ appartient à $C$; il s'ensuit que $l$ 'on a

$(\operatorname{Ad} \exp X-I)(\mathcal{S})=(\operatorname{Exp}$ ad $X-I)(S)=(0)$;

c'est-à-dire

(*) $\left(\right.$ ad $\left.x+\ldots+\frac{(\text { ad } x)^{r}}{r !}\right)(\rho)=(0)$.

Soit $Y$ un élément quelconque de $\mathcal{S}$; écrivons

$$
\text { ad } X(Y)=[X, Y]=\sum_{i=1}^{r} u^{i},
$$

avec $u^{i} \in m^{i}$. Comme on a

$$
\begin{aligned}
& (\text { ad } x)^{j}\left(m^{i}\right) \subset \mathcal{N}_{i+j}=m^{i+j} \boxplus \ldots \boxplus m^{r} \text { si } i+j \leqslant r \\
& (\operatorname{ad} x)^{j}\left(m^{i}\right)=(0)
\end{aligned}
$$

la relation (*) implique que $l^{\prime}$ on a nécessairement $u^{i}=0, \forall i \in\{1, \ldots, r\}$. Autrement dit $x$ appartient à $\mu_{b} \cap \xi$. On a donc prouvé que $c=\exp \left(\mu_{b} \cap \zeta\right) P$. Considérons à présent un élément $x$ de $\mu \cap \xi$ tel que exp $x$ normalise $P$. Nous avons

$$
(\operatorname{Exp} \text { ad } x-I)(Q) \subset Q \cap \mu
$$

c'est-à-dire

$$
\text { (**) } \quad\left(\operatorname{ad} x+\ldots+\frac{(\operatorname{ad} x)^{r}}{r !}\right)(\rho) \subset Q \cap \mu .
$$

Appelons $\Theta$ l'ensemble des poids de la représentation adjointe de $\mathcal{Q}$ dans $\boldsymbol{\zeta}^{\mathbb{C}}$; nous avons $\xi_{0}=S$ et $\xi^{\mathbb{C}}=\underset{\gamma \in \theta}{\boxplus} \zeta_{\gamma}$. Et par suite

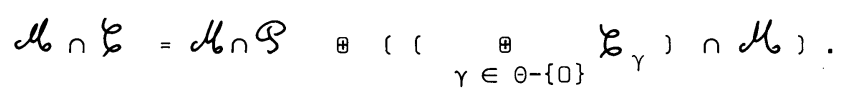

D'après (**), il est facile de voir que $X$ ne peut pas avoir une composante non nulle sur $\left(\underset{\gamma \in \theta-\{0\}}{\oplus} \xi_{\gamma}\right) \cap \mu$. Il s'ensuit donc que $x \in S \cap \mu$ et par suite $\exp x \in P$.

On en déduit que $P$ est son propre normalisateur dans $C$. Le lemme (1.13) est dẻmontré.

Dans le cas où $e g$ est une algèbre résoluble, nous avons $\mathcal{S}=(0)$ et $y=R=\xi$. Du lemme $(1.13)$ il résulte donc que l'on a :

Corollaire. - Les sous-groupes de Cartan ( [5]), d'un groupe de Lie résoluble connexe $\mathrm{R}$, sont les images par l'application exponentielle des sous-algèbres de Cartan de l'algèbre de Lie de $\mathrm{R}$. De plus tout sous-groupe de Cartan de $\mathrm{R}$ est son propre normalisateur. 
1.14. Lemme. - Soit $\Gamma^{\prime}$ le sous-groupe de $G$ formé des éléments qui normalisent à la fois les sous-groupes $N, A$, $K$ et $P$ ( et donc aussi $\Gamma$ et $\tilde{N}$ ). $\Gamma^{\prime}$ est un sous-groupe fermé de $G$ qui rencontre ses différentes composantes connexes. En outre nous avons $\Gamma^{\prime} \cap G_{0}=P \Gamma$.

Preuve. - Soit $Z\left(G_{0}\right)$ le centre de $G_{0}$. Les sous-groupes $N, A, K Z\left(G_{0}\right)$ et $P$ sont fermés dans $G$ ( voir ( 1.12 ) et les lemmes. ( 1.5 ) et (1.6). Comme $\Gamma$ ' est aussi I'intersection des normalisateurs dans $G$ de ces sous-groupes, $\Gamma$ ' est un sous-groupe fermé de $G$.

D'après la remarque ( 1.11 ), nous savons que deux décompositions du type

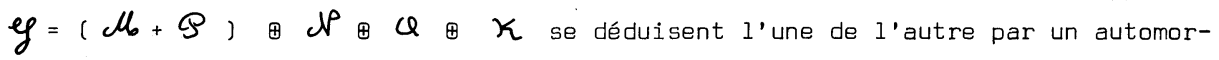
phisme de $y$ de la forme $A d x$, où $x$ appartient à $G_{0}$. Il s'ensuit donc que pour tout $g$ de $G$, il existe $g_{0} \in G_{0}$ tel que $g_{0}^{-1} g$ normalise à la fois $N$, $A$, $K$ et $P$. On en déduit que $\Gamma^{\prime}$ rencontre les différentes composantes connexes de $G$.

Soit $g \in \Gamma^{\prime} \cap G_{0}$; écrivons $g=r s$ avec $r \in R$ et $s \in S$. $r$ normalise $S$. Posons $[r, S]=\left\{r s^{\prime} r^{-1} s^{\prime-1}, s^{\prime} \in S\right\} ;[r, s]$ est contenu dans le sous-groupe discret $R \cap S$. Comme $S$ est connexe, il s'ensuit que $[r, S]=\{e\}$, c'est-àdire $r \in C$. L'élément $s$ normalise alors $N$, $A$ et $K$; nous avons donc $s \in \Gamma$. Puisque $S$ centralise $P, r$ normalise $P ; r$ appartenant à $C$, d'après le lemme ( 1.13 ) il s'ensuit que $r \in P$. Nous avons donc montré que $\Gamma^{\prime} \cap G_{0}=P \Gamma$.

1.15. Fin de la preuve de la proposition (1.7.). - Désignons par $Q$ le sous groupe $K \Gamma^{\prime}$ de $G$. D'après le lemme ( 1.14 ) nous avons $Q \cap G_{0}=P K$; il s'ensuit que $Q \cap G_{0}$ est la composante connexe de l'unité dans $Q$. D'autre part $P \Gamma=\Gamma^{\prime} \cap G_{0}$ est un sous-groupe fermé de $G$ ( lemme (1.14) ) et

$Q \cap G_{0} / P \Gamma=P K / P \Gamma$, qui est homéomorphe à $K / \Gamma$, est compact ( noter que $\Gamma$ contient le centre $Z(S)$ de $S$ ), on en déduit que $Q \cap G_{0}$ est un sousgroupe fermé de G. G ayant un nombre fini de composantes connexes, $Q$ est donc un sous-groupe fermé de $G$ possèdant le même nombre de composantes connexes que G.

Nous savons alors ( $[14]$, ch XV), théorème 3.7 ) que $Q$ possède des sousgroupes compacts maximaux deux à deux conjugués et, si $L$ est l'un d'eux, nous avons $Q=\left(Q \cap G_{0}\right) L$ ( et par suite $G=G_{0} L$, puisque $Q$ rencontre les différentes composantes connexes de $G$ ).

Choisissons un tel groupe $L$ et posons $K=K L$. Nous obtenons la décomposition $G=D K$, où $D$ est un sous-groupe résoluble connexe de $G$, l'image $A d K$ de $K$ par la représentation adjointe de $G$ est compacte et le sous-groupe $D \cap K$ est contenu dans $P$. Plus précisément nous avons : 
1.16. Lemme. - Soit $\mathrm{K}_{1}$ le plus grand sous-groupe compact du groupe de Lie nilpotent connexe $\mathrm{P}$ ([11]). Alors l'intersection des sous-groupes $D$ et $K$ est contenue dans $K_{1}(R \cap S)$ et donc dans $P$.

Preuve. - $L \cap G_{0}$ est un sous-groupe compact maximal de $Q \cap G_{0}=P K$. Il est clair que $L \cap G_{0}=K_{1}(K \cap L)$. Nous avons donc $D \cap K=D \cap\left(K\left(L \cap G_{0}\right)\right)=D \cap K_{1} K=D \cap\left(K_{1}(D \cap K)\right)$; or $D \cap K=R \cap S \subset R \cap Z\left(G_{0}\right) \subset C \cap Z\left(G_{0}\right) \subset P($ lemme (1.13) ). Le lemme ( 1.16 ) est prouvé. 


\section{FAMILLES DISCRIMINANTES D'A-COCYCLES ASSOCIEES A UN GROUPE DE LIE}

2.1. Définitions. - (voir [10]). Soient G un groupe L.C.D. et E un G - espace à droite (i.e. un espace topologique sur lequel $G$ opère à droite continûment). On appelle A-cocycle sur $E$ toute fonction continue $\rho$ sur ExG , à valeurs réelles vérifiant

$$
\rho\left(u, g_{1} g_{2}\right)=\rho\left(u, g_{1}\right)+\rho\left(u \cdot g_{1}, g_{2}\right) \text { pour } g_{1}, g_{2} \in G, u \in E
$$

Soient $L$ un sous-groupe de $G$ et $\rho$ un $A$-cocycle sur $E$; nous disons que $\rho$ est L-invariant si on a :

$$
\rho(u, 1)=0 \text {, pour } l \in L, u \in E
$$

Dorénavant $G$ désigne un groupe de Lie ayant un nombre fini de composantes connexes. Nous reprenons les notations du paragraphe précédent : En particulier on considère la décomposition $G=D K$, où $D$ est un sous-groupe fermé connexe réso-

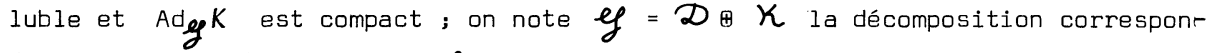
dante pour l'algèbre de Lie $\boldsymbol{f}$ de $G$.

2.2. Lemme. - G possède une frontière maximale (voir [1]), $\mathrm{B}(\mathrm{G})$, unique à $\mathrm{G}$ - homéomorphisme près. Le plus grand sous-groupe résoluble distingué $R$ ' de $G_{0}$ opère trivialement sur $\mathrm{B}(\mathrm{G}) ; \mathrm{B}(\mathrm{G})$ est alors un $\mathrm{R}^{\mathrm{G}}$ - espace (de fason évidente) qui est une frontière maximale du groupe de Lie $\mathrm{R}^{\mathrm{G}}$ dont la composante connexe est semi-simple et de centre trivial. En outre, B(G) est une frontière maximale de tout sous-groupe de $G$ contenant $G_{0}$.

Preuve. - Soit $N_{0}(D)$ (resp. $N(D)$ ) le normalisateur de $D$ dans $G_{0}$ (resp. dans $G$ ). Nous avons $N_{0}(D)=D \Gamma$ et $N(D)=D \Gamma$, (voir $(1.4)$ et prop. $(1.7)$

D'après [1], nous savons que $G_{0}$ possède une frontière maximale $B\left(G_{0}\right)$, unique à $G_{0}$ - homéomorphisme près, dont une version est $N_{0}(D) G_{0}$.

Soit $Z\left(G_{0}\right)$ le centre de $G_{0}$; $D Z\left(G_{0}\right)$ est un sous-groupe fermé (lemme $(1.6)$ ), distingué de $N(D)$. $D Z\left(G_{0}\right)^{N(D)}$ étant compact, d'après ([1] lemme II 8), N(D) possède la propiété de point fixe.

Comme $N(D)^{l^{G}}$ est $G_{0}$ - homéomorphe à $N_{0}(D)^{G_{0}}$, d'après ([1], corollaire 1 de la proposition II.2 ), on en déduit que $G$ possède une frontière maximale

$B(G)$, unique à $G$-homéomorphisme près, dont une version est $N(D) \backslash^{G}$.

D'autre part, désignons par $\xi^{\prime}$ l'application naturelle de $G$ sur $R^{\prime}{ }^{G}$. D'après ([1] lemme II.12) $R^{\prime}$ opère trivialement sur $B(G)$. B(G) est alors un $R^{\prime} I^{G}$ - espace (de façon évidente), $R^{I^{G}}$ - homéomorphe à

$$
\xi^{\prime}(N(D))=\xi^{\prime}(G) \text { qui est une frontière maximale de } R^{\prime}{ }^{G} \text {. }
$$

Enfin si $G_{1}$ est un sous-groupe de $G$ contenant $G_{0}$, notons $N_{1}(D)$ le norma- 
lisateur de $D$ dans $G_{1}$. Il est alors clair que la frontière maximale $N_{1}(D) V_{1}^{G}$ de $G_{1}$ est $G_{1}$ - homéomorphe à $N(D) l^{G}$ et donc à $B(G)$.

Le lemme 2.2. est prouvé.

2.3. Lemme. - Soit N(D) le normalisateur de $D$ dans $G$. Il existe sur la frontière maximale $\mathrm{B}(\mathrm{G})$ de $\mathrm{G}$ un unique élément $\mathrm{u}_{0}$ dont le stabilisateur dans $G$ est N(D).

Preuve. - $B(G)$ étant $G$ - homéomorphe à $N(D)^{G}$, il est clair qu'un tel point $u_{0}$ existe. Soit $u_{1}$ un autre point de $B(G)$ dont le stabilisateur est $N(D) ;$ écrivons $u_{1}=u_{0} \cdot x$, avec $x \in G$. L'élément $u_{0}$ est alors stable par les éléments de $x^{-1} N(D) x$; par suite nous avons $x^{-1} N(D) x \subset N(D)$; c'est-à-dire que $\times$ normalise $N(D)$.

Or N(D) est son propre normalisateur dans. $G$ : en effet, le normalisateur de $N(D)$ dans $G$ est une extension compacte de $N(D)$ et possède donc la propriété de point fixe ( $[1]$ lemme $I I .8$ ) ; mais $N(D)^{G}$ étant une frontière de $G$, on sait aussi ( [1] cor. 1 de la prop. II.2 ) que $N(D)$ est maximal pour la propriété de point fixe; par suite $N(D)$ est son propre normalisateur dans $G$. On en déduit alors que $x \in N(D)$ et $u_{1}=u_{0}$. Le lemme $(2.3)$ est prouvé.

2.4. Lemme. - Soit $G_{1}$ un sous-groupe (fermé) de $G$ contenant $G_{0}$. Désignons par $\zeta_{1}$ l'application naturelle de $G$ sur $G_{1}{ }^{G}$ et par $v_{0}$ l'unique élément $\left(u_{0}, \zeta_{1}(e)\right)$ de $B(G) \times G_{1} I^{G}$ stable par $G_{1} \cap N(D)$.

Alors l'application, $\rho \longmapsto \phi: d \in D \longmapsto \rho\left(v_{0}, d\right)$, établit une correspondance biunivoque entre les $A$ - cocycles $K$-invariants sur le G-espace homogène produit $\mathrm{B}(\mathrm{G}) \times \mathrm{G}_{1} \backslash^{\mathrm{G}}$ et les homomorphismes continus $\phi$ de $\mathrm{D}$ dans $\mathbb{R}$ additif teis que $\phi(k)=0$, pour tout $k \in K \cap D$ et $\phi\left(x^{-1} \mathrm{~d} x\right)=\phi(d)$, pour $d \in D, \quad x \in G_{1} \cap N(D)$.

Preuve. - Désignons par $\zeta_{1} l^{\prime}$ 'application naturelle de $G$ sur $G_{1}{ }^{G}$. L'élément $v_{0}=\left(u_{0}, \zeta_{1}(e)\right)$ de $B(G) \times G_{1} l^{G}$ est stable par $G_{1} \cap N(D)$. . Soit $\rho$ un $A$-cocycle K-invariant sur $B(G) \times G_{1}{ }^{G^{1}}$. Posons $\phi(d)=\rho\left(v_{0}, d\right),(d \in D)$; il est clair que l'on définit ainsi un homomorphisme continu $\phi$ de $D$ dans $\mathbb{R}$ additif vérifiant les propriétés énoncées. En outre, pour tout $(k, g) \in K \times G$, nous avons $\rho\left(v_{0} \cdot k, g\right)=\phi(d)$, où $d$ est un élément de $D$ tel que $k g=d k^{\prime}$ avec $k^{\prime} \in K$. On notera que $K$ est transitif sur $B(G) \times G_{1} \backslash^{G}$. 
Réciproquement, soit $\phi$ un homomorphisme continu de $D$ dans $\mathbb{R}$ additif possẹdant les propriétés énoncées. Posons

$$
\rho\left(v_{0} \cdot k, g\right)=\phi(d) \quad(k \in K, g \in G)
$$

où d est un élément de $D$ tel que $k g=d k^{\prime}$ avec $k^{\prime} \in K$. Puisque $\phi$ est nul sur $D \cap K$, la valeur donnée à $\rho\left(v_{0} \cdot k, g\right)$ ne dépend pas du choix de l'élément $d \in D$. D'autre part, soient $k_{1}, k_{2} \in K$ avec $v_{0} \cdot k_{1}=v_{0} \cdot k_{2}$ et $g \in G$; écrivons $k_{1} g=d_{1} k^{\prime}$ avec $d_{1} \in D, k_{1}^{\prime} \in K$, et $k_{2}=x k_{1}$ avec $x \in G_{1} \cap N(D) \cap K$; nous avons $k_{2} g=\left(x d_{1} x^{-1}\right) \times k_{1}^{\prime}$ et par suite

$$
\rho\left(v_{0} \cdot k_{2}, g\right)=\phi\left(x d_{1} x^{-1}\right)=\phi\left(d_{1}\right)=\rho\left(v_{0} \cdot k_{1}, g\right)
$$

Ce qui montre que $\rho$ est bien définie.

Montrons que $\rho$ est continue. Pour cela on est amené à montrer que si. $\left(g_{n}\right)$ est une suite d'éléments de $G$ convergeant vers un élément $g$ de $G$, on peut trouver une suite $\left(d_{n}\right)$ d'éléments de $D$ convergeant vers un élément $d$ de $D$ tels que $g_{n}=d_{n} k_{n}$ et $g=d k$ avec $k_{n}, k \in k$. Soit donc $\left(g_{n}\right)$ une telle suite; écrivons $g_{n}=d^{1} k_{n}^{1}$ et $g=d k$ avec $d{ }_{n}^{1}$, $d \in D$ et $k_{n}^{1}, k \in k$. la suite $x_{n}=d^{-1} g_{n} k^{-1}$ converge alors vers e; comme $\mathscr{y}=\mathcal{D} \boxplus \mathcal{H}$, nous pouvons trouver des suites $\left(d_{n}^{2}\right)$ et $\left(k_{n}{ }_{n}\right)$ d'éléments respectivement de $D$ et $K$ convergeant vers $e$, telles que $x_{n}=d_{n}^{2} k_{n}^{2}$. Il s'ensuit que l'on a :

$$
d_{n}^{2}=d^{-1} d_{n}^{1} z_{n} \text { et } k_{n}^{2}=z_{n}^{-1} k_{n}^{1} k^{-1} \text { avec } z_{n} \in D \cap k
$$

en posant $d_{n}=d_{n}^{1} z_{n}$ et $k_{n}=z_{n}^{-1} k_{n}^{1}$, on obtient ce que l'on voulait.

Enfin, on vérifie facilement les relations

$$
\begin{aligned}
& \rho(u, k)=0 \quad\left(u \in B(G) \times G_{1}{ }^{\prime}, \quad k \in K\right) \\
& \rho\left(u, g_{1} g_{2}\right)=\rho\left(u, g_{1}\right)+\rho\left(u, g_{1}, g_{2}\right)\left(u \in B(G) \times G_{1} i^{G} ; g_{1}, g_{2} \in G\right)
\end{aligned}
$$

2.5. Une famille d'homomorphismes de $\mathcal{D}$ dans $\mathbb{C}$ additif. - Soit $h$ le nilradical de $D$. Nous désignons par $\Xi$ l'ensemble des poids (voir ( 1.0 ) de la représentation adjointe, ${ }^{a d} D \mid h^{\mathbb{C}}$, de $D$ dans $\mathfrak{h}^{\mathbb{C}}$. ( $D$ étant résoluble, d'après le théorème de Lie, nous pouvons trouver une base de $\boldsymbol{\eta}^{\mathbb{C}}$ qui triangularise simultanément les éléments de ad $D / h^{\mathbb{C}}$. Alors $\Xi$ n'est autre que la famille d'homomorphismes définie par les éléments diagonaux ).

2.6. Lemme. - E engendre l'espace vectoriel complexe des homomorphismes de $D$ dans $\mathbb{C}$ additif qui s'annulent sur le nilradical $\boldsymbol{\xi}$ de $D$. La dimension de cet espace est donc égale à celle de l'espace vectoriel $D / h$.

Preuve. - Reprenons les notations du paragraphe 1 . Nous avons 
$D=R \oplus \mathcal{P} \oplus Q$. Désignons par $M$ le nilradical de $\mathcal{R}$ et par $\mathbb{S}$ une sous-algèbre de Cartan du centralisateur $\mathscr{\zeta}$ de la sous-algèbre de Lévi $\mathcal{S}$ de eg dans $\boldsymbol{R}$, Nous avons alors ( prop. $(1.7)$ ) $\boldsymbol{R}=\mu_{6}+\boldsymbol{S}$ et par suite $D=h+(\rho \otimes Q)$

où $h=M \otimes N$ est le nilradical de $D$.

Désignons par $\left(h_{i}\right), 1 \leqslant i \leqslant p$, la série centrale descendante de l'idéal nilpotent $h$ de $\mathcal{D}$. Il est clair que $\Xi$ est la réunion des poids des représentations adjointes de $D$ dans les espaces $\left(h_{i} / h_{i+1}\right)^{\mathbb{C}} \quad 1 \leqslant i \leqslant p$. Tout élément de $\Xi$ est donc nul sur $h$.

Considérons la représentation adjointe $\pi$ de $\mathcal{Q} \otimes Q$ dans $\xi^{\mathbb{C}}$. Il est clair que les poids de $\Pi$ sont les restrictions à $\rho \oplus Q$ des éléments de $\Xi$; nous identifions $\Xi$ et l'ensemble des poids de $\Pi$. Avec les notations de $(1.0)$, les sous-espaces $\xi_{\lambda}^{\mathbb{C}}, \lambda \in \Xi$, sont stables par $Q \boxplus G$ et nous avons

$$
h^{\mathbb{C}}=\underset{\lambda \in \Xi}{\oplus} \xi_{\lambda}^{\mathbb{C}}
$$

Soit alors $x \in \quad Q_{\boxplus} Q$ vérifiant $\lambda(x)=0$ pour tout $\lambda \in \Xi$. Pour tout $\lambda \in \Xi$ et tout $Y \in \zeta_{\lambda}^{\mathbb{C}}$, il existe un entier $n$ tel que $(\operatorname{ad} X)^{n}(Y)=0$. Autrement dit ad $x$ est nilpotent sur $\xi$. Comme $D=\xi+(g \otimes Q)$, il s'ensuit que ad $X$ est nilpotent sur $D$ et par suite $X$ appartient au nilradical $\xi$ de $D$. Le lemme $(2.6)$ est prouvé..

2.7. Corollaire. - Soit G un groupe de lie ayant un nombre fini de composantes connexes dont la composante connexe $G_{0}$ de l'unité est semi-simple. Soit $y=\mathcal{P} \boxplus Q \boxplus K$ une décomposition d'Iwasawa de l'algèbre de lie ef de $G$. Alors la famille $\Xi$ d'homomorphismes de $\mathcal{P} \boxplus Q$ dans $\mathbf{c}$ additif, obtenue en $(2.5$ ), engendre tout l'espace des homomorphismes de $\mathcal{P} \boxplus Q$ dans $\mathbf{C}$ additif. La dimension de cet espace est égale à celle de $Q$.

Preuve. - Nous avons

$$
\mathcal{P}=[\mathcal{P} \oplus Q, \mathcal{P} \otimes Q] ;
$$

par suite tout homomorphisme de $\mathcal{P} \boxplus Q$ dans $\mathbb{C}$ additif, s'annule sur le nilradical $\mathcal{P}$ de $\mathcal{P} \boxplus Q$. Le corollaire résulte alors du lemme $(2.6)$.

2.8. Lemme. - Soit $\theta$ un automorphisme de $y$ laissant stable $D$. Alors pour tout $\lambda$ de $\Xi, \lambda \circ \theta \in \Xi ; \theta$ permute ainsi les éléments de $\Xi$. En particulier $\lambda \circ A d x=\lambda$ pour tout $\lambda \in \Xi$ et tout $x \in N_{0}(D)$.

Preuve. - $\theta$ est un automorphisme de $y$ laissant stable $D, \theta$ laisse donc invariant le nilradical $\mathcal{h}$ de $D$. 
Soit $\lambda \in \Xi$. Il existe alors un idéal $\mathscr{O}^{\prime}$ de $D$, contenu dans $h$, et un élément $v$ de $h$ - $\mathcal{O}^{2}$ vérifiant

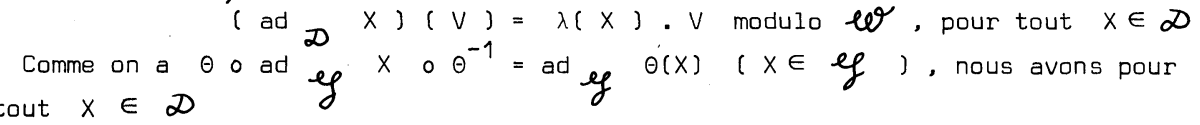

$$
\left(\text { ad } \begin{array}{rl}
\theta(x))(\theta(V)) & =(\theta 0 \text { ad } D(X))(V) \\
& =\lambda(X) \cdot \theta(V) \text { modulo } \theta(\mathcal{C}) ;
\end{array}\right.
$$

c'est-à-dire pour tout $x \in D$

ce qui montre que $\lambda \cdot 0^{-1} \in \Xi$

$$
\text { ( ad } D x)(\theta(V))=\lambda \circ \theta^{-1}(x) \text {. } \theta(V) \text { modulo } \theta \text { ( eV) ; }
$$

D étant connexe, il s'ensuit alors que $\lambda \circ \mathrm{Ad} x=\lambda$, pour tout $\lambda \in \Xi$ et tout $x \in D$. Enfin comme $\Gamma$ centralise $P$ et $A$, la restriction $A d \gamma \mid \rho \oplus Q$, de Ad $\gamma$ à $P \oplus Q$ est l'identité, pour tout $\gamma$ de $\Gamma$; il s'ensuit que $\lambda \circ$ Ad $\gamma=\lambda$ pour tout $\lambda \in \Gamma$ et pour tout $\lambda \in \Xi$. Comme $N_{0}(D)=D \Gamma$, le lemme ( 2.8 ) est prouvé .

\subsection{Une famille d'A-cocycles K-invariants sur la frontière maximale $B(G)$} de G. - Soit $\lambda \in \Xi ; D$ étant un groupe résoluble connexe $\lambda$ est la différentielle en e d'un poids $\phi_{\lambda}$ de la représentation adjointe de $D$ dans $\xi^{\mathbb{C}}$. Nous avons alors :

i) pour tout automorphisme $\sigma$ de $G$ laissant stable $D, \phi_{\lambda} \sigma \sigma=\phi_{\lambda} 0_{d \sigma}$ ( lemme ( 2.8$)$ ). En particulier $\phi_{\lambda}\left(x d x^{-1}\right)=\phi_{\lambda}(d)$, pour $d \in D$ et $x \in N_{0}(D)$.

ii) $\phi_{\lambda}$ prend la valeur 1 pour tout élémént du nilradical MN, de $D$. De plus plus, comme $\operatorname{Ad}_{y} K$ est compact, $\left|\phi_{\lambda}(k)\right|=1$, pour tout $k \in D \cap K$.

D'après le lemme ( 2.4 ) à l'homomorphisme continu log $\left|\phi_{\lambda}\right|$ de $D$ dans $\mathbb{R}$ additif, se trouve associé un A-cocycle K-invariant $\tau_{\lambda}$ sur $B(G) \times G_{0} \backslash G$. Le normalisateur $N$ (D) de $D$ dans $G$ rencontre les différentes composantes connexes de $G$; soit $s$ une section de l'application naturelle $\zeta$ de $G$ sur $G_{0} \backslash G$, à valeur dans $N(D)$. Alors $l$ 'homomorphisme continu de $D$ dans $\mathbb{R}$ additif, $\frac{1}{\text { card } G_{0}{ }^{G}} \quad x \in \sum_{G_{0}}{ }^{G} \log \mid \phi_{\lambda} \circ$ Ad $s(x) \mid$ est invariant par les automorphismes intérieurs associés, non seulement aux éléments de $N_{0}(D)$, mais aussi à ceux de $N(D)$. D'après le lemme ( 2.4 ) à cet homomorphisme se trouve donc associé un A-cocycle $K$-invariant $\rho_{\lambda}$ sur $B(G)$.

Nous avons,

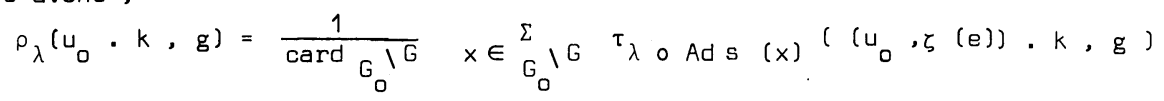

$(k \in K, g \in G)$,

où $u_{0}$ est l'élément de $B(G)$ stable par $N(D)$ (lemme ( 2.3 )); c'est-à- 
dire, aussi. $\rho_{\lambda}(u, g)=\int_{G{ }_{0} G} \tau_{\lambda}((u, x), g) d x(u \in B(G), g \in G)$, où $d x$ désigne la mesure de Haar normalisée sur le groupe fini $G_{0} \backslash G$.

Nous avons ainsi associé à $G$ une famille d'A-cocycles $\left\{\rho_{\lambda}, \lambda \in \Xi\right\}$ sur la frontière maximale $B(G)$. Il est facile de voir que cette famille ne dépend de la décomposition $G=D K$ que par le choix du sous-groupe $K$; c'est-à-dire que pour toute décomposition $G=D^{\prime} K$ où $D^{\prime}$ est un conjugué de $D$, on obtient la même famille d'A-cocycles $K$-invariants sur $B(G)$.

2.10. Remarque. - D'après le lemme ( 2.6 ), la famille d'A-cocycles K-invariants sur $B(G), \quad\left\{\rho_{\lambda}, \lambda \in \Xi\right\}$ obtenue en ( 2.9 ) engendre l'espace vectoriel des A-cocycles, $\rho, K$-invariants sur $B(G)$ tels que $l$ 'homomorphisme de $D$ dans $\mathbb{R}$ additif, $d \longmapsto \rho\left(u_{0}, d\right),(\operatorname{voir} l e m m e(2.4)$ ), est nul sur le nilradical de $D$. Cet espace vectoriel est donc de dimension inférieure ou égale à celle de $\boldsymbol{b}^{\mathbb{D}}$. [ I l peut en effet arriver qu'un élément de $\Xi$ soit à partie réelle nulle sans être nul ].

Si $G_{0}$ est semi-simpie, il résulte du corollaire ( 2.6 ) que la famille d'A-cocycles ainsi obtenue engendre tout l'espace vectoriel des A-cocycles $K$-invariants sur $B(G)$. Cet espace vectoriel est donc de dimension égale à celle de $\boldsymbol{Q}$. [ En effet, dans ce cas, les éléments de $\Xi$ sont réels ( voir (1.2) ] ].

2.11. Définition. - A tout sous-groupe $K$ qui provient d'une décomposition de $G$ du type construit au paragraphe 1 , nous avons associé ( $\operatorname{voir}(2.9$ ) ) une famille d'A-cocycles, K-invariants, $\left\{\rho_{\lambda}, \lambda \in \Xi\right\}$, sur la frontière maximale $B(G)$ de $G$. Une telle famille sera appelée une famille discriminante d'A-cocycles associée à $G$.

Si $x$ est un élément de $G$, on note $I_{x}$ l'automorphisme intérieur associé à $x^{-1}$ (i.e. $I_{x}: g \longmapsto x^{-1} g x$ ) et $J_{x} l$ 'homéomorphisme de $B(G)$ sur luimême qui à $u \in B(G)$ associe $u \cdot x$. Nous avons alors

2.12. Lemme. - Soit $\left\{\rho_{\lambda}, \lambda \in \Xi\right\}$ une famille discriminante d'A-cocycles associée d̀ G. Alors toute autre telle famille s'écrit $\left\{\rho_{\lambda} \circ\left(J_{d}, I_{d}\right), \lambda \in \Xi\right\}$ pour un certain élément $d$ de $D$.

Preuve. - Considérons la décomposition $G=D K$ de $G$ et soit $K^{\prime}$ un quelconque sous-groupe de $G$ qui provient d'une décomposition analogue de $G$. Alors $G$ admet la décomposition $G=D K^{\prime}$ et $K^{\prime}$ est le conjugué de $K$ par un élément d 
de $D$ ( i.e. $K^{\prime}=d K d^{-1}$ ).

Il est alors clair qu'à partir de la décomposition $G=D K^{\prime}$, on obtient ( voir ( 2.9 ) ) la famille d'A-cocycles $K^{\prime}$-invariants sur $B(G)$ $\left\{\rho_{\lambda} \circ\left(J_{d}, I_{d}\right\}, \lambda \in \Xi\right\}$. Il suffit en effet de remarquer que si $x \in G$, la composante sur $D$ de $d \times d^{-1}$, pour la décomposition $G=D K$, est la conjuguée par d de la composante sur $D$ de $x$, pour la décomposition $G=D K$. Le lemme 2.12 est prouvé.

En passant, on notera que l'on a $\rho_{\lambda} \circ\left(J_{d}, I_{d}\right)(u, g)=\rho_{\lambda}(u, g)+\rho_{\lambda}(u, g, d)-\rho_{\lambda}(u, d)$ $(u \in B(G), g \in G)$

2.13. Remarque. - Appelons $\Xi_{0}$ l'ensemble des poids de la représentation adjointe de $D$ dans $\boldsymbol{h}^{\mathbb{C}} /[\boldsymbol{\xi}, \boldsymbol{\xi}]^{\mathbb{C}} ; \Xi_{0}$ est un sous-ensemble de $\Xi$. Comme on sait que tout système $x_{1}, \ldots . x_{s}$ d'éléments de $\boldsymbol{h} \mathbb{C}$ dont l'image dans $\boldsymbol{h}^{\mathbb{C}}[\boldsymbol{\xi}, \boldsymbol{\xi}]^{\mathbb{C}}$ forme une base de cet espace, engendre $\xi^{\mathbb{C}}$ en tant qu'algèbre de Lie, il est facile de voir que tout élément $\lambda$ de $\Xi$ est une somme d'éléments de $\Xi_{0}$. Il s'ensuit alors que tout élément de $\left\{\rho_{\lambda}, \lambda \in \Xi\right\}$ est une somme d'éléments de $\left\{\rho_{\lambda}, \lambda \in \Xi_{0}\right\}$. 


\section{ALGEBRES DE LIE NILPOTENTES}

3.1. Définition. - Soit $G$ un groupe topologique, $A_{1}, \ldots, A_{r}(r \in \mathbb{N})$ des sousgroupes fermés de $G$; nous disons que $G$ est le produit amalgamé des sous-groupes $A_{1}, \ldots A_{r}$ si l'application du produit cartésien $A_{1} \times \ldots \times A_{r}$ dans $G$ qui au couple $\left(a_{1}, \ldots, a_{r}\right)$ fait correspondre le produit $a_{1} \ldots a_{r}$ est un homéomorphisme. Dans ce cas les applications $\rho_{1}, \ldots, \rho_{r}$ qui à un élément $g$ de $G$ font correspondre les éléments $\rho_{1}(g), \ldots, \rho_{r}(g)$ respectivement de $A_{1}, \ldots, A_{r}$ tels que $g=\rho_{1}(g) \ldots \rho_{r}(g)$, sont appelés les projections de $G$ respectivement sur $A_{1}, A_{2}, \ldots, A_{r}$.

Remarques. - Il est facile de voir que

i) Soient $G$ un groupe localement compact qui est une réunion dénombrable de compacts, A et $B$ des sous-groupes fermés de $G$; alors si l'application $\psi: A \times B \longrightarrow G$ est surjective, elle est ouverte; side plus on $a A \cap B=\{e\}$, (a,b) $\longmapsto a b$

$G$ est le produit amalgamé des sous-groupes $A$ et $B$.

ii) Soit $G$ un groupe de Lie qui est le produit amalgamé de deux sous-groupes fermés $A$ et $B$; alors l'application $\psi$ est un isomorphisme de variétés analytiques.

Dans la suite, nous utiliserons le résultat suivant :

3.2. Lemme. - Soit G un groupe de Lie connexe dont l'algèbre de Lie y est somme directe de deux sous-algèbres $g_{1}$ et $g_{2}$. Soient $G_{1}$ et $G_{2}$ des sous-groupes de Lie de $G$ ayant respectivement $\boldsymbol{f}_{1}$ et $\boldsymbol{y}_{2}$ pour algèbre de Lie. Si tout élément de $G$ s'écrit de facon unique comme produit d'éléments de $G_{1}$ et $G_{2}$, alors $G$ est le produit amalgamé de $G_{1}$ et $G_{2}$.. En particulier $G_{1}$ et $G_{2}$ sont fermés dans $G$.

Preuve. - D'après les hypothèses l'application $\eta: G_{1} \times G_{2} \longrightarrow G$ est bijective. Comme $\mathscr{f}=\mathscr{g}_{1} \oplus \mathscr{f}_{2}$, nous savons que $n$ est un isomorphisme de variétés analytiques d'un ouvert $V \times W$ contenant $(e, e)$ de $G_{1} \times G_{2}$ sur un ouvert $U$ contenant e de $G$ (e désigne l'élément unité de $G$ ). Soit alors $g=g_{1} g_{2} \in G$ avec $g_{1} \in G_{1}$ et $g_{2} \in G_{2}$. La restriction de $n^{-1}$ à l'ouvert $x=g_{1} U g_{2}$, contenant $g$, se décompose en

$$
\begin{aligned}
& x \longrightarrow v \frac{n^{-1}}{x \longmapsto} v \times w \longrightarrow G_{-} \times G_{+} \\
& x g_{1}^{-1} \times g_{2}^{-1}(v, w) \longrightarrow\left(g_{1} v, w g_{2}\right) .
\end{aligned}
$$


et est donc analytique. L'application $n$ est donc un isomorphisme de variété analytique de $G_{1} \times G_{2}$ sur $G$; autrement dit $G$ est le produit amalgamé des sous-groupes fermés $G_{1}$ et $G_{2}$ '.

Soit $h$ une algèbre de Lie nilpotente réelle ou complexe de dimension $\mathrm{m}$, $h_{1}=\xi, h_{2}=\left[\xi, h_{1}\right], \ldots, h_{p}=\left[\xi, h_{p-1}\right], h_{p+1}=(0)$, sa série centrale descendante. Soit $e_{1}, \ldots, e_{i_{1}}, e_{i_{1}+1}, \ldots, e_{i_{p}}=e_{m}$ une base de $h$ adaptée à cette série (i.e. pour tout $j=1, \ldots, m, e_{j} \in \zeta_{\ell} \mid \zeta_{\ell+1}$. si $i_{\ell-1}<j \leqslant i_{\ell}$ ). La formule de Campbell-Hausdorff ,

$$
u \times v=u+v+\frac{1}{2}[u, v]+\ldots \quad(u, v \in \xi),
$$

permet de munir $h$ d'une structure de groupe nilpotent.

Désignons par $A$ l'algèbre des fonctions polynomiales sur l'espace vectoriel $h$. Soient $\left(x_{i}\right)_{1 \leqslant i \leqslant m}$ le système de fonctions coordonnées associées à la base $\left(e_{i}\right)_{1 \leqslant i \leqslant m}$; on définit ([12]) une notion de degré sur $\mathcal{A}$ en attribuant un degré à chaque générateur $x_{i}$; le degré de $x_{i}$, noté "dg $x_{i}$ ", est par définition égal au plus grand entier $s$ tel que $e_{i} \in \xi_{s}$; on convient que le degré du polynome nul est $(-\infty)$. Cette notion est évidemment indépendante du choix de la base adaptée. Si $u \in \boldsymbol{h}$, on pose $x_{i}(u)=u_{i}$. On sait que la multiplication dans $\boldsymbol{h}$ est polynomiale. Plus précisément on a, pour tout $u$ et $v \in \xi$.

$$
\forall i \in\left\{1, \ldots, i_{1}\right\} \quad, \quad(u \times v)_{i}=u_{i}+v_{i}
$$

$\forall \ell \in\{2, \ldots, p\}, \forall i \in\left\{i_{\ell-1}+1, \ldots, i_{\ell}\right\},(u \times v)_{i}=u_{i}+v_{i}+T_{i}\left(u_{1}, \ldots, u_{i} ; v_{\ell-1}, \ldots, v_{i_{\ell-1}}\right)$

où les $T_{i}$ sont des polynômes dont le degré total est inférieur ou égal à $l$ et le degré partiel par rapport à l'ensemble des variables $u_{k}$ (resp. $v_{k}$ ), $1 \leqslant k \leqslant i_{\ell-1}$ est inférieur ou égal à $(\ell-1)$.

Si $c \in \xi$, nous désignons par $L_{C}$ (resp. $R_{C}$ ) la multiplication à gauche (resp. à droite) par c dans $\boldsymbol{h}$. Il résulte de $(3.4$.$) que L_{c}$ et $R_{c}$ sont des applications polynomiales. Un endomorphisme $E$ de l'espace vectoriel $\zeta$ est dit adapté à sa série centrale si les $h_{\ell}, 1 \leqslant \ell \leqslant P$, sont stables par $E$.

3.5. Lemme. - Avec les notations ci-dessus, nous avons:

i) $\forall \mathrm{c} \in \boldsymbol{\xi}, \forall \mathrm{T} \in \mathcal{R}, \mathrm{T} \circ \mathrm{L}_{\mathrm{c}}=\mathrm{T}+\mathrm{T}^{\prime}$ avec $\mathrm{dg} \mathrm{T}^{\prime}<\mathrm{dg} \mathrm{T}$.

ii) $S i \mathrm{E}$ est un endomorphisme (resp. un automorphisme) d'espace vectoriel de $h$, adapté à sa série centrale, alors

$\forall T \in \mathcal{R} \quad \operatorname{dg}(T o E) \leqslant \operatorname{dg} T \quad$ (resp. $\operatorname{dg}(\mathrm{ToE})=\operatorname{dg} T)$. 
Preuve. - Il suffit de montrer le lemme quand $T$ est un générateur $x_{i}$ de $R$. La première partie est alors une conséquence immédiate de (3.4.). Quant à la seconde, $E$ étant adapté, nous avons

$$
E\left(e_{j}\right)=\sum_{\ell=1}^{m} E_{j, \ell} e_{\ell} \text { avec } E_{j, \ell}=0 \text { si } d g x_{j}>d g x_{\ell} .
$$

D' où

$x_{i} \circ E(u)=\sum_{j=1}^{m} u_{j} x_{i} \circ E\left(e_{j}\right)=\sum_{j=1}^{m} E_{j, i} u_{j}=\sum_{\left\{j: d g x_{j} \leqslant d g x_{i}\right\}} E_{j, i} u_{j} \quad(u \in h)$

qui montre que $d g\left(x_{i}\right.$ oE $) \leqslant d g x_{i}$.

Enfin si E est un automomorphisme, cette dernière inégalité appliquée à E et $E^{-1}$ donne l'égalité des degrés.

3.6. Lemme. - Soient $\boldsymbol{Q}$ et $\boldsymbol{B}$ deux sous-azgèbres de $\boldsymbol{h}$ telles que

$$
h_{l}=\left(a \cap h_{l}\right) \otimes\left(B \cap \cap \zeta_{l}\right), 1 \leqslant l \leqslant p .
$$

Alors le groupe nilpotent $(h, x)$ est le produit amalgamé des sous-groupes $(Q, x)$ et $(B, x)$. De plus les projections (au sens de (3.1.)) de $\xi$ sur $a$ et $B$ sont polynomiales.

Preuve. - Elle se fait par récurrence sur la longueur de la série centrale de $h$. Si $\xi$ est abélienne la proposition est évidente. Supposons donc qu'elle soit vérifiée pour les algèbres de Lie nilpotentes de longueur strictement inférieure à $k$ et soit $\boldsymbol{h}$ de longueur $k$. Considérons alors $\xi_{k}$, l'homomorphisme $I 1$ de $\boldsymbol{h}$ sur $h h_{k}$ et une section linéaire o de I telle que o o Ir $(Q) \subset Q$ et $\sigma \circ \Pi(\mathbb{B}) \subset \mathbb{B}$. L'hypothèse de récurrence s'applique à $h / h_{k}$ : pour tout élément $u$ de $\xi$, l'élément $\Pi(u)$ de $h_{/} h_{k}$ s'écrit donc de façon unique comme produit d'élément de $\Pi(Q)$ et $\Pi(B)$; de plus

$$
\Pi(u)=\bar{\rho}_{1}(\Pi(u)) \times \bar{\rho}_{2}(\Pi(u)),
$$
où $\bar{\rho}_{1}$ et $\bar{\rho}_{2}$ sont des applications polynomiales de $\bar{\eta} / \xi_{k}$ respectivement sur
$\Pi(Q)$ et $\Pi(B)$.

Posons alors $\tau_{1}(u)=\sigma \circ \rho_{1} \circ \Pi(u)$ et $\tau_{2}(u)=\sigma \circ \rho_{2} \circ \Pi(u)$; l'élément $y=\tau_{1}(u) \times \tau_{2}(u)$ a même image que $u$ par $\pi$, il s'ensuit que $z=u-y$, appartient à $\hat{h}_{k}$ qui est contenu dans le centre de $\boldsymbol{h}$. Mais

$$
\text { - } h_{k}=\left(a \cap h_{k}\right) \otimes\left(B \cap h_{k}\right)=\left(a \cap h_{k}\right) \times\left(\infty \cap h_{k}\right) ;
$$

et si $P_{Q}$ (resp. $P_{B}$ ) désigne la projection de $\zeta_{k}$ sur $Q \cap \zeta_{k}$ (resp. $B \cap h_{k}{ }^{\top}$ il vient,

$$
u=\tau_{1}(u) \times \tau_{2}(u)+P_{Q}(z)+P_{B}(z)
$$


où $P_{Q}(z)$ et $P_{B}(z)$ appartiennent au centre de $\boldsymbol{b}$.

D'où $u=\tau_{1}(u) \times \tau_{2}(u) \times P_{Q}(z) \times P_{B 3}(z)$

$=\left(\tau_{1}(u) \times P_{Q}(z)\right) \times\left(\tau_{2}(u) \times P_{\infty}(z)\right)$
$=\left(\tau_{1}(u)+P_{Q}(z)\right) \times\left(\tau_{2}(u)+P_{\infty}(z)\right)$.

En posant

$$
\begin{aligned}
& \rho_{1}(u)=\tau_{1}(u)+p_{\boldsymbol{e}}\left(u-\tau_{1}(u) \times \tau_{2}(u)\right) \\
& \rho_{2}(u)=\tau_{2}(u)+p_{Q}\left(u-\tau_{1}(u) \times \tau_{2}(u)\right),
\end{aligned}
$$

$\rho_{1}$ et $\rho_{2}$ sont des applications polynomiales et tout élément $u$ de $\xi$ s'écrit de façon unique comme produit d'éléments de $Q$ et $\boldsymbol{B}$ sous la forme

$$
u=\rho_{1}(u) \times \rho_{2}(u) \cdot
$$

Remarque. - Cette démonstration montre en fait que si on a seulement

$$
h_{\ell}=\left(a \cap \zeta_{\ell}\right)+\left(B \cap \zeta_{l}\right), \quad \ell \in\{1, \ldots, p\} \text {, }
$$

(sommes non nécessairement directes), il existe des applications $\rho_{1}$ et $\rho_{2}$ polynomiales de $\boldsymbol{h}$ sur $\boldsymbol{Q}$ et $\boldsymbol{B}$ telles que $u=\rho_{1}(u) \times \rho_{2}(u) \quad \forall u \in \boldsymbol{\xi}$. Le couple $\left(\rho_{1}, \rho_{2}\right)$ n'est pas unique si $\boldsymbol{Q} \cap \boldsymbol{B} \neq(0)$.

3.7. Soit $\mathcal{L}$ (resp. $\boldsymbol{\zeta}$ ) une sous-algèbre nilpotente (resp. un idéal nilpotent) d'une algèbre de Lie réelle $\boldsymbol{y}$.

Considérons la représentation adjointe de $\mathcal{L}$ dans l'idéal $\mathfrak{f}^{\mathbb{C}}$ de $\mathcal{g}^{\mathbb{C}}$ et désignons par $\Theta$ l'ensemble de ses poids. Nous avons ([25]), avec les notations de (1.0.),

i) Les sous-espaces $\xi_{\theta}^{\mathbb{C}}$ sont stables par $\mathscr{L}$

$$
\xi^{\mathbb{C}}=\stackrel{\oplus}{\in} \theta \stackrel{\mathbb{C}}{\theta}
$$

$$
\left[\xi_{\theta}^{\mathbb{C}}, \xi_{\theta^{\prime}}^{\mathbb{C}}\right] \subset \xi_{\theta+\theta^{\prime}}^{\mathbb{C}}, \quad\left(\theta, \theta^{\prime} \in \theta\right)
$$

(avec la convention $\boldsymbol{\zeta}_{\theta+\theta^{\prime}}^{\mathbb{C}}=(0)$ si $\theta+\theta^{\prime} \notin \theta$ ).

Définition. - Une partitiọn $\left\{\Theta_{-}, \theta_{+}\right\}$de $\theta$ est dite stable par addition et conjugaison si :

a) Pour tout $\theta \in \theta_{-}$(resp. $\theta_{+}$) la forme linéaire conjuguée $\bar{\theta}$ appartient à $\theta_{-}$ (resp. $\theta_{+}$) .

b) $\left.\begin{array}{l}\theta+\theta^{\prime} \in \theta \\ \theta, \theta^{\prime} \in \theta_{-} \quad\left(\text { resp. } \theta_{+}\right)\end{array}\right\} \Longrightarrow \theta+\theta^{\prime} \in \theta_{-}\left(\right.$resp. $\left.\theta_{+}\right)$.

c) Dans le cas où la forme linéaire nulle appartient à $\theta$, on convient qu'elle appartient à $\theta_{+}$. 
Soit $\left\{\theta_{-}, \theta_{+}\right\}$une telle partition de $\theta$ et posons

$$
\boldsymbol{\zeta}_{-}^{\mathbb{C}}={ }_{\theta}^{\oplus} \theta_{-} \boldsymbol{\eta}_{\theta}^{\mathbb{C}}, \quad \boldsymbol{\zeta}_{+}^{\mathbb{C}}=\theta_{\theta}^{\oplus} \theta_{+} \boldsymbol{\zeta}_{\theta}^{\mathbb{C}} .
$$

En vertu de iiil et de l'hypothèse b), $\boldsymbol{h}^{\mathbb{C}}$ et $h_{+}^{\mathbb{a}}$ sont des sous-algèbres de $\xi^{\mathbb{C}}$. De l'hypothèse a), il résulte que $\xi_{-}^{\mathbb{C}}$ et $\xi_{+}^{\mathbb{C}}$ sont les complexifiées de deux sous-algèbres $\boldsymbol{h}_{\text {- et }} \boldsymbol{h}$ + de $\boldsymbol{\zeta}^{-}$. A toute partition $\left\{\Theta_{-}, \theta_{+}\right\}$de $\theta$ stable par addition et conjugaison, nous avons donc associé une décomposition de $\zeta$ en somme directe de deux sous-algèbres $\xi$ - et $\xi_{+}$.

Soit $\boldsymbol{M}$ un idéal nilpotent de $\boldsymbol{g}$ contenu dans $\boldsymbol{h}$. Appelons $\Lambda$ l'ensemble des poids de la représentation adjointe de $\mathcal{L}$ dans $\boldsymbol{M}^{\mathbb{C}}$. $\Lambda$ est un sous-ensemble de $\Theta$. A toute partition $\left\{\Theta_{-}, \theta_{+}\right\}$de $\theta$ stable par addition et conjugaison, correspond la partition $\left\{\Lambda \cap \Theta_{-}, \Lambda \cap \Theta_{+}\right\}$de $\Lambda$ stable par addition et conjugaison; notons $\boldsymbol{H}_{\boldsymbol{C}}=\boldsymbol{\mu}_{-} \oplus \boldsymbol{\mu}_{+}$la décomposition de $\boldsymbol{\mu}_{\boldsymbol{C}}$ en somme directe de deux algèbres associée à cette dernière. Il est alors clair que l'on a :

$$
\mu_{-}=\mu_{b} \cap h_{\text {- et }} \mu_{+}=\mu_{0} \cap h_{+} \text {. }
$$

Soit $\left(\boldsymbol{\zeta}_{i}\right)_{1 \leqslant i \leqslant p}$ la série centrale descendante de l'idéal nilpotent $\boldsymbol{h}$. Pour tout $i \in\{1, \ldots, p\}, \boldsymbol{h}_{i}$ est un idéal nilpotent de $\boldsymbol{g}$ contenu dans $\boldsymbol{h}$. Nous avons donc d'après ce qui précède (en faisant $\boldsymbol{H}_{\boldsymbol{b}}=\boldsymbol{\zeta}_{i}, 1 \leqslant i \leqslant p$ ),

$$
\xi_{i}=\left(\xi_{i} \cap \zeta_{-}\right) \oplus\left(\xi_{i} \cap \xi_{+}\right), 1 \leqslant i \leqslant p .
$$

D'après le lemme (3.6.), il s'ensuit que muni du produit $x$ définit par la formule (3.3.), $h$ est un groupe nilpotent qui est le produit amalgamé des sousgroupes $\xi_{\text {- }}$ et $\xi_{+}$. De plus les projections associées à ce produit amalgamé sont polynomiales. 
4.0. - Nous reprenons les notàtions du paragraphe 1 : on désigne par

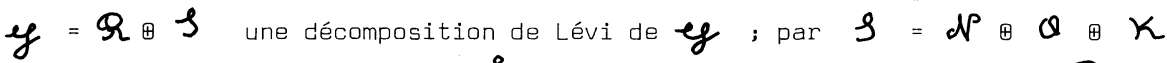
une décomposition d'Iwasawa de $\mathcal{S}$; on obtient la décomposition $\mathcal{J}=D \oplus$, où $D=\Omega \oplus \mathcal{R} \otimes Q$ est une sous-algèbre résoluble et $\boldsymbol{K}$ une sous-algèbre compacte de $\mathrm{eg}$.

Soit $\boldsymbol{\mu}$ le nilradical de $\mathcal{R}$ et $\mathcal{S}$ une sous-algèbre de Cartan du centralisateur $\boldsymbol{b}$ de $\boldsymbol{S}$ dans $\boldsymbol{R}$. Nous avons (prop. $(1.7)$ ) $\boldsymbol{R}=\mu_{0}+\boldsymbol{\rho}$ et par suite

où $\boldsymbol{H}=\mathscr{M} \otimes \mathcal{P}$ est le nilradical de $\boldsymbol{D}$.

E désigne l'ensemble des poids de la représentation adjointe de $D$ dans $\boldsymbol{h}^{\mathbb{C}}$. 4.1. - Décomposition de $\varnothing$ et ef associée à une partition de $\Xi$. Considérons la représentation adjointe $I$ de $\mathcal{P} Q \boldsymbol{Q}$ dans $\boldsymbol{H}^{\mathbb{C}}$. Tout élément de $\Xi$ est nul sur $\mathcal{H}$ et sa restriction à $\boldsymbol{Q} \mathbb{Q}$ fournit un poids de $\Pi$; nous identifions $\Xi$ et l'ensemble des poids de $\Pi$. Soit $\left\{\Xi_{-}, \Xi_{+}\right\}$une partition de $\Xi$ stable par addition et conjugaison; notons $\boldsymbol{\eta}=\boldsymbol{\eta}_{-} \rightarrow \boldsymbol{H}_{+}$la décomposition de $\boldsymbol{f}$ associée à cette partition (voir ( 3.7$)$ ).

Posons $D_{-}=\xi_{-}$et $D_{+}=\left(\xi_{+}+Q\right) \oplus Q$. Des relations $[S, S]=(0),\left[S^{-} \otimes, h_{-}\right] \subset \xi_{-}, \quad\left[S \otimes Q, h_{+}\right] \subset h_{+}$et $[9, Q] \subset \xi \cap \xi_{0}^{\mathbb{C}} \subset \xi_{+}$, il résulte que $\boldsymbol{D}_{-}$et $\boldsymbol{D}_{+}$sont des sous-algèbres de ఐ. Nous obtenons ainsi les décompositions

$$
D=D_{-} \oplus D_{+} \text {et } \boldsymbol{y}=D_{-} \oplus \xi_{+},
$$

où $\xi_{+}=D_{+} \oplus K$ est un sous-espace de $\boldsymbol{g}$.

4.2. Proposition. - Soit $G$ un groupe de Lie ayant un nombre fini de composantes connexes. Désignons par $\boldsymbol{y}$ l'algèbre de Lie de $G$; par $\boldsymbol{y}=\boldsymbol{R} \boldsymbol{\rho}$ une décomposition de Lévi de $\boldsymbol{g}$ et par $\mathcal{S}=\mathcal{P}_{\boxplus} Q \mathbb{R}$ une décomposition d'Iwasawa de $\mathcal{J}$. Nous obtenons la décomposition $\boldsymbol{g}=\boldsymbol{D} \boxplus \boldsymbol{K}$, où $\Phi=\boldsymbol{\Omega} \oplus \mathcal{N} \boxplus \boldsymbol{Q}$ est une sous-algèbre résoluble et $\boldsymbol{K}$ une sous-algèbre compacte de $\mathrm{e}$.

Appezons $\Xi$ l'ensemble des poids de la représentation adjointe de $D$ dans la complexifiée $\boldsymbol{\xi}^{\mathbb{C}}$ de son nilradical $\xi^{\text {. Soit }}\left\{\Xi_{-}, \Xi_{+}\right\}$une partition stable par addition et conjuguaison de $\Xi$ et notons $D=\boldsymbol{D}_{-} \oplus \boldsymbol{D}_{+}$la décomposition de $\boldsymbol{D}$ associée à cette partition (voir ( 4.1$)$ ). 
Désignons par N, A, K, P, $\mathrm{D}_{-}$et $\mathrm{D}_{+}$les sous-groupes de Lie connexes de $G$ ayant respectivement $\mathcal{P}, \boldsymbol{Q}, \boldsymbol{K}, \boldsymbol{S}, \mathcal{D}_{-}$et $\mathcal{D}_{+}$pour algèbres de Lie. Soit $\Gamma^{\prime}$ le sous-groupe fermé de G formé des éléments qui normalisent à la fois $N, A, K$ et $P$ (voir prop. (1.7)). Posons $E_{+}=D_{+} K \Gamma^{\prime} ; E_{+}$est une sous-variété de $\mathrm{G}$, qui la réunion d'un nombre fini de sous-variétés ouvertes isomorphes à $D_{+} K$.

Alors l'application qui au couple ( $a, b)$ associe le produit ab est un isomorphisme de variétés analytiques de $\mathrm{D}_{-} \times \mathrm{E}_{+}$sur $\mathrm{G}$. En outre $\mathrm{D}_{-}$est nilpotent simplement connexe.

Désignons par D le sous-groupe de Lie connexe de $G$ ayant $D$ pour algèbre de Lie. Pour prouver la proposition ( 4.2 ) nous commençons par établir la proposition :

4.3. Proposition. - D est le produit amalgamé (déf. ( 3.1 )) des sous-groupes

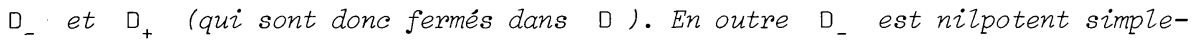
ment connexe.

Preuve. - Désignons par M, P, A et $N$ les sous-groupes de Lie connexes de G ayant respectivement $\boldsymbol{H}, \boldsymbol{Q}, \boldsymbol{Q}$ et $\mathcal{P}$ pour algèbre de Lie. Il est clair que l'on a

$$
D_{-}=\exp \xi_{-} \text {et } D_{+}=\left(\exp \xi_{+}\right) P A \text {. }
$$

MN est le nilradical de $D$. Nous savons que ( 3.7 ) le nilradical $h$ de $D$ est, pour le produit $\times$ défini par la formule ( 3.3 ), le produit amalgamé des sous-groupes $\xi_{-}$et $h_{+}$; de la formule de Campbell-Hausdorff, il s'ensuit que l'on a

$$
M N=\exp \xi=\left(\exp \xi_{-}\right)\left(\exp \xi_{+}\right)
$$

L'application analytique $n: D_{-} \times D_{+} \longrightarrow D$ est donc surjective (puisque

$$
\left(d_{-}, d_{+}\right) \longrightarrow d_{-} d_{+}
$$

$D=M N P A]$.

Remarquons à présent que :

i) si $x \in h_{-}$, ad $x$ (et par suite (Ad $g$ - I) pour $g \in D_{-}$) envoie chaque $h_{\gamma}^{\mathbb{C}}, \gamma \in \Xi$, dans la somme directe des $\boldsymbol{h}_{\gamma^{\prime}}^{\mathbb{C}}$, tels que $\gamma^{\prime}-\gamma_{\gamma} \Xi_{-}$et $Q \otimes Q$ dans $\boldsymbol{h}_{-}(\operatorname{voir}(3.7))$.

ii) Si $x \in h_{+}$, ad $x$ (et par suite (Ad $g-I$ ) pour $g \in D_{+}$) envoie chaque $h_{\gamma}^{\mathbb{C}}, \gamma \in \Xi$, dans la somme directe des $h_{\gamma}^{\mathbb{C}}$, tels que $\gamma^{\prime}-\gamma \in \Xi_{+} \cup\{0\}$ et $Q \oplus Q$ dans $\eta_{+}$.

iii) Si $x \in \xi$ est tel que $\exp x$ appartient au centre de $D$, nous avons Ad $\left.(\exp x)\right|_{\boldsymbol{D}}=\left.\operatorname{Exp}(\operatorname{ad} x)\right|_{\boldsymbol{D}}=I$; d'où ad $\left.x\right|_{D}=0$ (i.e. $x$ appartient au 
centre de $D$ ) et par suite $x \in \xi \cap \xi_{0}^{\mathbb{a}} \subset \xi_{+}$.

De ces trois remarques il résulte que $D_{-} \cap D_{+}=\{e\}$ : en effet si $g \in D_{-} \cap D_{+}$, des remarques $i$ ) et $i$ il il résulte que $A d g=I$; d'après $i i i)$ nous avons alors nécessairement $\mathrm{g}=\mathrm{e}$. On en déduit que I'application $\mathrm{n}$ est bijective.

D'après le lemme' ( 3.2 ), D est alors le produit amalgamé des sous-groupes fermés $D_{-}$et $D_{+}$.

Montrons que $D_{\text {_ }}$ est simplement connexe. On sait ([11]) que tout groupe de Lie nilpotent connexe possède un plus grand sous-groupe compact qui est le plus grand sous-groupe compact du centre et qui est tel que le groupe quotient correspondant soit simplement connexe. De plus comme le centre d'un groupe de Lie nilpotent connexe est connexe $([14]$, ch. XVI, th. 1.1 ), ce sous-groupe compact maximum est donc connexe.

Appelons $K_{1}$ (resp. $K_{2}$ ) le sous-groupe compact maximum de $D_{-}$(resp. du nilradical $M N$ de $D$ ). Nous avons $K_{1} \subset K_{2}$; pour montrer que $D_{\text {_ }}$ est simplement connexe on est amené à montrer que $K_{1}$ est réduit à $\{e\}$.

Le groupe des automorphismes du tore $k_{2}$ est discret ( $[14]$, ch. III, prop. 3.2 ) . Comme $K_{2}$ est distingué dans $D$ et $D$ est connexe, il s'ensuit que $K_{2}$ est contenu dans le centre de $D$. D'après la remarque iii) on a alors nécessairement $K_{2} \subset D_{+}$et par suite $K_{1}=\{e\}$. La proposition est prouvée.

4.4. Preuve de la proposition ( 4.2 ) - D'après la proposition ( 4.3 ) l'application $n: D_{-} \times E_{+} \longrightarrow G$ est surjective (puisque $G=D K \Gamma^{\prime}$ ).

$$
(a, b) \longmapsto a b
$$

En outre nous avons :

$$
D \cap K \Gamma^{\prime}=D \cap P K=D \cap(P(K \cap D))=P
$$

(puisque $D \cap K \subset D \cap K \subset P$ (prop. $(1.7)$ )). On en déduit que $n$ est injective : en effet soit $d_{-}\left(d_{+} k\right)=d_{-}^{\prime}\left(d_{+}^{\prime} k^{\prime}\right)$ avec $d_{-}, d_{-}^{\prime} \in D_{-}, d_{+}, d_{+}^{\prime} \in D_{+}$et $k, k^{\prime} \in K \Gamma^{\prime}$; nous avons $d_{-} d_{+}=d_{-}^{\prime} d_{+}^{\prime} x$ avec $x \in D \cap K \Gamma^{\prime} \subset P \subset D_{+} ; d^{\prime}$ où $d_{-}^{-1} d_{-}^{\prime} \in D_{-} \cap D_{+}=\{e\}$ et par suite $d_{-}=d_{-}^{\prime}, d_{+} k=d_{+}^{\prime} k^{\prime}$.

Comme $y=D_{-} \oplus D_{+} \boxplus K, l$ 'application $n$ est un isomorphisme de variétés analytiques d'un voisinage $V \times W$ de $D_{-} \times E_{+}$contenant $(e, e)$ sur un ouvert $U$ de $G$ contenant $e$. Soit $g=d K \in G$ avec $d \in D$ et $k \in K \Gamma^{\prime}$. La restriction de $n^{-1}$ à l'ouvert $X=d U k$, contenant $g$, se décompose en

$$
\begin{aligned}
& x \longrightarrow v \stackrel{n^{-1}}{\longrightarrow} v \times w \longrightarrow D_{-} \times E_{+} \\
& x \longmapsto d^{-1} \times k^{-1}(v, w) \longmapsto\left(\rho_{-}(d v), \rho_{+}(d v) w k\right),
\end{aligned}
$$

où $\rho_{\text {_ }}$ et $\rho_{+}$désignent les projections associées au produit amalgamé $D=D_{-} D_{+}$ 
(proposition ( 4.3 )). Il s'ensuit que la restriction de $n^{-1}$ à l'ouvert $x$ est analytique. Par suite $n$ est un isomorphisme de variétés analytiques de $D_{-} \times E_{+}$ sur G. La proposition ( 4.2 ) est démontrée.

4.5. Remarque. - Soit $y=\left(\mu_{+} \varphi^{1}\right) \oplus \mathcal{N}^{1} \oplus Q^{1} \oplus \boldsymbol{K}^{1}$ une décomposition de $\mathcal{g}$ obtenue par le même procédé que celle $\boldsymbol{y}=(\boldsymbol{\mu}+\boldsymbol{\rho}) \oplus \mathcal{N} \oplus Q \oplus K$. Nous savons (remarque ( 1.11 )) que la nouvelle décomposition se déduit de l'ancienne par un automorphisme intérieur, Ad $x, x \in G_{0}$. La famille $\Xi$ est alors remplacée par la famille d'homomorphismes de $\boldsymbol{D}^{1}=\boldsymbol{R}^{\oplus} \mathbb{P}^{1} \oplus \boldsymbol{Q}^{1}$ dans $\mathbb{C}$ additif, $\Xi^{1}=\left\{\lambda \circ \mathrm{Ad} \mathrm{x}^{-1}, \lambda \in \Xi\right\}$. Posons $\Xi_{-}^{1}=\left\{\lambda \circ \mathrm{Ad} \mathrm{x}^{-1}, \lambda \in \Xi_{-}\right\}$et $\Xi_{+}^{1}=\left\{\lambda \circ \mathrm{Ad} x^{-1}, \lambda \in \Xi_{+}\right\}$. La décomposition de $\mathcal{g}$ (resp. G) associée à la partition $\left\{\Xi_{-}^{1}, \Xi_{+}^{1}\right\}$ se déduit de celle associée à la partition $\left\{\Xi_{-}, \Xi_{+}\right\}$par l'automorphisme intérieur de $\boldsymbol{g}$ (resp. de $G$ ) associé à $x$. 
5.0.- Nous reprenons les notations du paragraphe 1 : on considère les décompositions $y=R_{\oplus} S$ (Lévi), $S=P \oplus Q \oplus K$ (Iwasawa) et $S=\mathcal{P} \oplus Q \oplus \tilde{\mathcal{D}} \oplus \mathbf{M}$ (Bruhat). On pose $D=R_{\boxplus} Q_{\boxplus} d P$. On désigne par $\Sigma$ l'ensemble des poids de la représentation adjointe de $\boldsymbol{Q}$ dans $\mathcal{S}$; par $W$ la chambre de Weyl de $\boldsymbol{Q}$ qui a servi à définir $\mathcal{N}$ et par $\Sigma_{1}$ (resp. $\Sigma_{2}=-\Sigma_{1}$ ) l'ensemble des éléments de $\Sigma$ négatifs (resp. positifs) sur $W$. On note $\mathcal{H}$ le nilradical de $\mathcal{R}$ et on choisit une sous-algèbre de $\operatorname{Cartan} \mathcal{S}$ du centralisateur de $\mathcal{S}$ dans $\boldsymbol{R}$. Nous avons (voir $(4.0))$

$$
D=\xi+\left(Q_{\oplus} Q\right)
$$

où $\zeta=M \oplus d P$ est le nilradical de $D$.

On désigne par $R, N, A, \tilde{N}, K, P, S$ et $D$ les sous-groupes de Lie connexes de $G$ ayant respectivement $\mathscr{R}, \mathcal{P}, \mathcal{Q}, \widetilde{\mathcal{P}}, K, \mathcal{S}, \mathcal{S}$ et $D$ pour algèbre de Lie. On note $\Gamma$ le centralisateur de $A$ dans $K$ et $\Gamma^{\prime}$ l'intersection des normalisateurs des sous-groupes $N, A, K$ et $P$.

On appelle $N_{0}(D)$ et $N(D)$ les normalisateurs de $D$ respectivement dans $G_{0}$ et $G$; nous avons $N_{0}(D)=D \Gamma$ et $N(D)=D \Gamma^{\prime}$.

Soit $\Theta$ l'ensemble des poids de la représentation adjointe de $\mathcal{S} Q \boldsymbol{Q}$ dans $\boldsymbol{y}^{\mathbb{C}}$. Comme $[\boldsymbol{S}, \boldsymbol{S}]=(0), \sum$ s'identifie à un sous ensemble de $\theta$. Pour tout $\theta \in \theta$, nous notons $\boldsymbol{y}_{\theta}^{\mathbb{C}}\left(\operatorname{voir}(1.0)\right.$ ) le sous-espace de $\boldsymbol{y}^{\mathbb{C}}$ formé des éléments qui pour chaque $H$ de $Q \oplus Q$ sont annulés par une puissance de (ad $H-\theta(H) I)$.

5.1. Définitions. - Nous disons qu'une partition $\left\{\theta_{-}, \theta_{+}\right\}$de $\theta$ est adaptée à la chambre de Weyl $W$ si elle est stable par addition et conjuguaison, et si $\Sigma_{1}$ (resp. $\Sigma_{2}=-\Sigma_{1}$ ) est contenu dans $\theta_{-}$(resp. $\theta_{+}$) :

En outre nous disons que $\left\{\theta_{-}, \theta_{+}\right\}$est stable par automorphismes intérieurs si pour tout $\gamma$ de $\theta_{-}$(resp. $\theta_{+}$), $\gamma$ o Adg appartient à $\theta_{-}$(resp. $\theta_{+}$), pour tout élément $g$ du normalisateur $N(D)$ de $D$ dans $G$. Si $G$ est connexe cette définition est trivialement satisfaite (voir lemme ( 2.8 )).

5.2. Proposition. - Avec les notations de (5.0), soit $\left\{\theta_{-}, \theta_{+}\right\}$une partition de $\Theta$ adaptée à la chambre de Weyl $W$ et stable par automorphismes intérieurs. Posons

$$
y_{-}^{\mathbb{C}}=\underset{\theta \in \theta_{-}}{\mathbb{P}} \boldsymbol{y}_{\theta}^{\mathbb{C}} \text { et } y_{+}^{\mathbb{C}}=\underset{\theta \in \theta_{+}}{\mathbb{P}} \boldsymbol{y}_{\theta}^{\mathbb{C}} \text {. }
$$

Alors $y_{-}^{\mathbb{C}}$ et $y_{+}^{\mathbb{C}}$ sont deux sous-algèbres de $y^{\mathbb{C}}$ qui sont les complexifiées de 
deux sous-algèbres $y_{-}$et $y_{+}$de $y$ telles que $y_{y} y_{-} \boxplus y_{+}$.

$D^{\prime a u t r e}$ part désignons par $\left(G_{0}\right)_{-}$et $\left(G_{0}\right)_{+}$les sous-groupes de Lie connexes

de $G_{0}$ ayant respectivement $\boldsymbol{y}_{-}$et $\boldsymbol{y}_{+}$pour algèbres de Lie et posons $G_{-}=\left(G_{0}\right)_{-}, G_{+}=\left(G_{0}\right)_{+} \Gamma^{\prime}$. Alors $G_{-}$et $G_{+}$sont des sous-groupes fermés de $G$ et l'application qui au couple $\left(\mathrm{g}_{-}, \mathrm{g}_{+}\right)$associe le produit $\mathrm{g}_{-} \mathrm{g}_{+}$est un isomorphisme de variétés analytiques de $G_{-} \times G_{+}$sur une sous-variété ouverte de $G$ dont le complémentaire est de mesure de Haar nulle.

Dans le cas semi-simple nous obtenons :

5.3. Proposition. - Soit $S$ un groupe de Lie ayant un nombre fini de composantes connexes dont la composante connexe de l'unité est semi-simple. Alors avec les notations de $(5.0)$, l'application qui à $(n, a, \gamma, \tilde{n})$ associe le produit $n$ a $\gamma \tilde{n}$ est un isomorphisme de variétés analytiques de $N \times A \times \Gamma^{\prime} \times \tilde{N}$ sur une sous-variété ouverte de $\mathrm{S}$ dont le complémentaire est de mesure de Haar nulle.

Dans le cas d'un groupe de Lie semi-simple connexe de centre fini ce résultat est bien connu (lemme de Bruhat, voir par exemple [13], p. 47).

Les sections de ( 5.4 ) à ( 5.7 ) sont consacrées à la preuve de la proposition ( 5.2 ). Nous commençons par établir une proposition et un lemme.

5.4. Proposition. - L'application qui au couple $(g, x)$ associe le produit $g x$ est un isomorphisme de variétés analytiques de $N(D) \times \tilde{N}$ sur une sous-variété ouverte de $G$ dont le complémentaire est de mesure de Haar nulle.

Preuve. - Notons $d$ 'abord que $N(D) \cap \tilde{N}=\{e\}$ : en effet soit $x \in N(D) \cap \tilde{N}$, écrivons $x=\exp x$ avec $x \in \tilde{\mathcal{N}}$. Puisque $x$ normalise $D$ et $[a, \tilde{\mathcal{P}}] \subset \tilde{\mathcal{N}}$, il s'ensuit que

$$
(A d x-I)(\varphi)=(\text { Exp ad } x-I)(Q) \subset D \cap \tilde{\mathcal{P}}=(0) \text {. }
$$

En reprenant alors un raisonnement déjà utilisé pour prouver le lemme $(1.13)$, on montre que $l$ 'on a $[x, a]=(0)$. Autrement dit $x \in \mathcal{S}_{0} \cap \tilde{\mathcal{P}}=(0)$ (voir $(1.2))$; d'où $x=e$.

L'algèbre de Lie du groupe de Lie $N(D)$ est $D \boxplus m$. Comme on a

$$
e=D \otimes m \otimes \widetilde{P} \text {; }
$$

il est clair que l'application qui au couple $(g, x)$ associe le produit $g x$ est un isomorphisme de variétés analytiques de $N(D) \times \tilde{N}$ sur une sous-variétés ouverte de $G$.

Il reste donc à prouver que le complémentaire dans $G$ de la sous-variété ouverte 
N(D) $\tilde{N}$ est de mesure de Haar nulle. Comme N(D) $\tilde{N}$ est une réunion finie de sousvariétés ouvertes isomorphes à $N_{0}(D) \tilde{N}$, on est amené à prouver que le complémentaire dans $G_{0}$ de la sous-variété ouverte $N_{0}(D) \tilde{N}$ est de mesure de Haar nulle. Soit $R^{\prime}$ l'image réciproque du centre de $R^{V^{G}}$ o par l'application naturelle de $G_{0} \operatorname{sur} R^{G_{0}}$. $R^{\prime} l^{G_{0}}$ est un groupe de Lie connexe, semi-simple, de centre trivial. Nous avons $R^{\prime}=R Z(S)$, où $Z(S)$ désigne le centre de $S$; par suite $R^{\prime}$ est contenu dans $R \Gamma$ et donc dans $N_{0}(D)$. Si $\xi^{\prime}$ désigne l'application naturelle de $G_{0}$ sur $R^{\prime}{ }^{G_{0}}$, il s'ensuit alors que le complémentaire dans $G_{0}$ de $N_{0}(D) \tilde{N}$ est I'image réciproque par $\xi^{\prime}$ du complémentaire dans $R^{\prime} I^{G}$ de $\xi^{\prime}\left(N_{0}(D) ~ \tilde{N}\right)$. Comme un borélien $B$ de $R, \backslash G_{O}$ est de mesure de Haar nulle si et seulement si le borélien $\xi^{\prime^{-1}}(B)$ de $G_{0}$ est de mesure de Haar nulle, on est ramené à montrer la propriété dans le cas où $G$ est un groupe de Lie connexe semi-simple de centre trivial.

La proposition ( 5.4 ) résulte alors du lemme de Bruhat ([13], p. 47).

5.5.- Désignons par $\Xi(r e s p$. $\Lambda$ ) l'ensemble des poids de la représentation adjointe de $D$ dans $\boldsymbol{h}^{\mathbb{C}}$ (resp. dans $\mathcal{M}^{\mathbb{C}}$ ). $\Xi$ (resp. $\Lambda$ ) s'identifie à l'ensemble des poids de la représentation adjointe de $\mathcal{S} \oplus \boldsymbol{U}$ dans $\boldsymbol{h}^{\mathbb{C}}$ (resp. dans $\boldsymbol{H}^{\mathbb{C}}$ ) (voir ( 4.1$)$ ). $\Xi$ et $\Lambda$ s'identifient donc à des sous ensembles de $\theta$ et nous avons

$$
\Xi=\Lambda \cup \Sigma_{1}, \quad \theta=\Xi U \Sigma_{2} U\{0\}=\Lambda U \Sigma \text {. }
$$

5.6. Lemme. - Pour tout élément $\lambda$ et $\gamma$ respectivement de $\Lambda$ et $\Sigma$, nous avons, avec les notations de (1.0):

$$
\left[\mathcal{H}_{\lambda}^{\mathbb{L}}, \rho_{\gamma}\right] \subset \mathcal{M}_{\lambda}^{\mathbb{C}},
$$

où $\lambda^{\prime}$ est un élément de $\Lambda$ tel que la restriction à $S$ (resp. à $Q$ ) de la forme linéaire $\lambda^{\prime}-\lambda$ est nulle (resp. égale à $\gamma$ ).

Pour tout automorphisme $\theta$ de $y$ laissant stable $\mathcal{S} \otimes C$ nous avons

$$
\boldsymbol{\mu}_{\lambda \circ \theta^{-1}}^{\mathbb{C}}=\theta\left(\boldsymbol{M}_{\lambda}^{\mathbb{C}}\right) \quad(\lambda \in \Lambda) \text {. }
$$

Preuve. - Notons $\tilde{\gamma}$ la forme linéaire sur $\mathscr{S} \otimes Q$ dont la restriction à $Q$ (resp. à $\boldsymbol{Q}$ ) est nulle (resp. égale à $\gamma$ ). Compte tenu que l'on a $[\boldsymbol{S}, \mathcal{S}]=(0)$, la première assertion du lemme découle de la formule de Leibnitz : $(\operatorname{ad} x-(\lambda(x)+\tilde{\gamma}(x)) I)^{n}([v, w])=\sum_{k=0}^{n} c_{n}^{k}\left[(\operatorname{ad} x-\lambda(x) I)^{k}(v),(\operatorname{ad} x-\tilde{\gamma}(x) I)^{n-k}(w)\right]$, avec $x \in Q \oplus Q, v$ et $w \in \boldsymbol{y}^{\mathbb{C}}$. 
D'autre part, soient $\lambda \in \Lambda$ et $v \in \mathcal{M}_{\lambda}^{\mathbb{C}}$. Il existe un entier $n$ tel que

$$
(\operatorname{ad} x-\lambda(x) I)^{n}(v)=0, \quad \forall x \in S_{\boxplus} Q \text {. }
$$

Comme on a

$$
\theta \circ \quad \dot{a d x} \circ \theta^{-1}=\operatorname{ad} \theta(x) \quad(x \in \boldsymbol{g}) \text {, }
$$

nous avons pour tout $x \in \rho_{\boxplus} Q$,

$$
\begin{aligned}
0 & =\theta 0(\operatorname{ad} x-\lambda(X) I)^{n}(v)=(\operatorname{ad} \theta(X)-\lambda(X) I)^{n} \theta(v) \\
& =\left(\operatorname{ad} \theta(x)-\lambda \circ \theta^{-1}(\theta(x)) I\right)^{n} \theta(v) .
\end{aligned}
$$

D'où (ad $\left.Y-\lambda \circ \theta^{-1}(Y) I\right)^{n} \theta(V)=0, \forall Y \in Q_{\boxplus} Q$; ce qui prouve que $\theta(v) \in \mu_{\lambda \circ \theta^{-1}}^{\mathbb{C}}$. Le lemme est démontré .

5.7. Preuve de la proposition ( 5.2 ). - Reprenons les notations de la proposition ( 5.2$\}$. Les partitions $\left\{\Lambda \cap \theta_{-}, \Lambda \cap \theta_{+}\right\}$et $\left\{\Xi \cap \theta_{-}, \Xi \cap \theta_{+}\right\}$respectivement de $\Lambda$ et $\Xi$ sont stables par addition et conjugaison. Désignons respectivement par $\mu_{=} \mu_{-} \oplus \mu_{+}$et $D=D_{-} \boxplus D_{+}$les décompositions de $\mu_{\text {et }} D$ associées à ces partitions (voir ( 3.7 ) et ( 4.1$)$ ).

La partition $\left\{\Theta_{-}, \theta_{+}\right\}$de $\theta$ étant adaptée à la chambre de Weyl $W$, nous avons : $\theta_{-}=\theta_{-} \cap \Xi$ : et $\theta_{+}=\left(\theta_{+} \cap \equiv\right) \cup \Sigma_{2} \cup\{0\}$, d'après ( 5.5 );

$\left[\mathcal{N}_{\oplus} m, \mu_{-}\right] \subset \mu_{-}$et $\left[\tilde{\mathcal{N}}_{\oplus} m, \mu_{+}\right] \subset \mu_{+}$, d'après le lemme ( 5.6 ); en outre $\mathcal{P}$ est contenu dans $\mathcal{D}_{-}$. car $\Sigma_{1}$ est contenu dans $\theta_{-}=\theta_{-} \cap \Xi$.

Nous avons alors

et

$$
y_{-}=D_{-}=M_{-} \oplus \mathcal{N}, D_{+}=\left(\mu_{+}+\Phi\right) \oplus Q
$$

$$
y_{+}=D_{+} \oplus \tilde{\mathcal{N}}_{\oplus} \mathrm{m} .
$$

Désignons par $D_{-}$et $D_{+}$les sous-groupes de Lie connexes de $G$ ayant respectivement $\boldsymbol{D}_{-}$et $\boldsymbol{D}_{+}$pour algèbre de Lie; nous avons $D_{-}=\left(\exp \boldsymbol{\mu}_{-}\right) \mathrm{N}$ et $D_{+}=\left(\exp \bar{\mu}_{+}\right) P A$. D'après la proposition ( 4.3$)$, D est le produit amalgamé des sous-groupes $D_{-}$et $D_{+}$. Nous avons alors

$$
G_{-}=\left(G_{0}\right)_{-}=D_{-},\left(G_{0^{+}}\right)_{+}=D_{+} \tilde{N} \Gamma \text { et } G_{+}=D_{+} \tilde{N} \Gamma^{\prime} \text {. }
$$

Comme la partition $\left\{\theta_{-}, \theta_{+}\right\}$de $\theta$ est stable par automorphismes intérieurs, $\Gamma^{\prime}$ normalise (outre $N, A, K, \tilde{N}$ et $P$ ) les sous-groupes (exp $\mu_{-}$) et $\left(\exp \mu_{+}\right)$(lemme ( 5.6$\left.)\right) ; G_{+}$est donc un sous-groupe de $G$.

Des relations

$N(D) \cap \tilde{N}=e(\operatorname{prop} .(5.4)), D_{-} \cap D_{+}=e$ et $D \cap \Gamma^{\prime}=P \subset D_{+}(\operatorname{prop} .(1.7))$, il résulte que $G_{-} \cap G_{+}=e \cdot G_{-}$et $G_{+}$sont des sous-groupes de Lie de $G$ ayant pour algèbre de Lie $\mathscr{y}_{-}$et $\boldsymbol{y}_{+}$; comme $\mathscr{y}_{=} \boldsymbol{y}_{-} \boxplus \mathscr{y}_{+}$, il est alors clair que 
l'application qui au couple ( $g_{-}, g_{+}$) associe le produit $g_{-} g_{+}$est un isomorphisme de variétés analytiques de $G_{-} \times G_{+}$sur la sous-variété ouverte $N(D) \tilde{N}$ de $G$ dont le complémentaire est de mesure de Haar nulle (proposition ( 5.4 )).

Pour achever la.preuve de la proposition ( 5.2 ), il nous reste à prouver que $G_{+}$est un sous-groupe fermé de $G$.

$G$ ayant un nombre fini de composantes connexes, il suffit de prouver que $\left(G_{0}\right)_{+}=D_{+} \tilde{N} \Gamma$ est fermé dans $G_{0}$.

Soit $\xi^{\prime}$ l'application naturelle de $G_{0}$ sur $R^{\prime} l^{G_{O}} ; R^{\prime} l^{G_{0}}$ est un groupe de Lie connexe semi-simple de centre trivial et nous savons que $\xi^{\prime}(N A \Gamma)$ est fermé dans $R^{\prime} I^{G}$. On en déduit que $R A \tilde{N} \Gamma$ est fermé dans $G_{0}$ (noter que $R^{\prime} C R \Gamma$ ). Comme il est clair que $R A \tilde{N} \Gamma$ est le produit amalgamé des sous-groupes (exp $\mu_{-}$) et $D_{+} \tilde{N} \Gamma$, il s'ensuit que $D_{+} \tilde{N} \Gamma$ est fermé dans $G_{0}$.

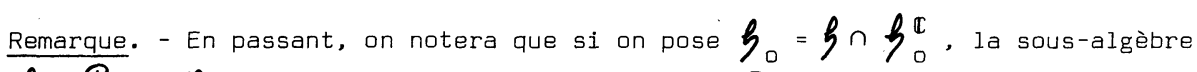
$\overline{(\xi+P)} \oplus Q$ est une sous-algèbre de Cartan de $D$. Dans ce qui précède on pour-

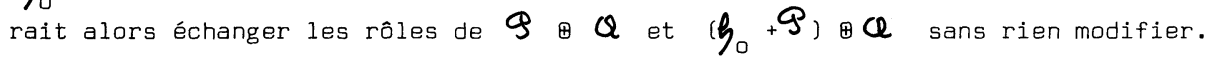


6. THEORIE DE LA MESURE ET FONCTIONS HARMONIQUES

Nous désignons par $G$ un groupe localement compact à base dénombrable (L.C.D.) et par e son élément unité.

\section{G-espaces et théorie de la mesure. -}

6.1.-Soient $E$ et $F$ deux espaces topologiques (resp. deux espaces de Banach) sur lesquels $G$ opère; nous disons qu'une application $\phi$ de $E$ sur $F$ est un $G$ homéomorphisme (resp. une G-isométrie) si c'est un homéomorphisme (resp. une isométrie) commutant avec les opérations de $G$. Nous appelons G-espace à gauche (resp. à droite), tout espace topologique $E$ sur lequel $G$ opère à gauche (resp. à droite) continûment.

Soit $E$ un espace localement compact, nous notons C(E) l'espace des fonctions continues bornées réelles sur E muni de la norme de la convergence uniforme, $M^{1}(E) \quad l$ 'ensemble des mesures de probabilité sur $E$ (i.e. l'ensemble des mesures de Radon positives de masse totale 1). La masse unité en un point $x$ est un élément de $M^{1}(E)$ noté $\varepsilon_{x}$. L'intégrale d'une fonction borélienne $f$ sur $E$ par rapport à une mesure $\mu$ de $M^{1}(E)$ sera notée indifféremment $\int f(x) \mu(d x)$ ou $\langle\mu, f>$.

Soit $E$ un G-espace à gauche localement compact, $\mu \in M^{1}(G)$ et $\lambda \in M^{1}(E)$. Nous appelons convolution $\mu * \lambda$ de $\mu$ et $\lambda$, l'élément de $M^{1}(E)$ défini par :

$$
\langle\mu * \lambda, f\rangle=\int_{G} \int_{E} f(g \cdot x) \mu(d g) \lambda(d x) \quad \begin{aligned}
& \text { (f fonction borélienne positive } \\
& \text { sur } E) .
\end{aligned}
$$

Lorsque $\mu=\varepsilon_{g}(g \in G)$ nous notons $g \lambda$ la mesure $\varepsilon_{g}^{* \lambda}$. On note $\mu^{n}$ le produit de convolution $\mu * \ldots * \mu$ (n facteurs) sur $G$.

6.2.-Soit $\mu \in M^{1}(G)$ et $\lambda \in M^{1}(E)$; nous disons que $\lambda$ est $\mu$-invariante si $\mu * \lambda=\lambda$.

Une mesure $m$ de $M^{1}(E)$ est dite quasi-invariante si les mesures $m$ et gm sont équivalentes pour tout $g$ de $G$. Si $E$ est un $G$-espace homogène, il existe des mesures quasi-invariantes sur $E$ et deux quelconques d'entr'elles sont équivalentes ([19]); nous notons alors $L^{\infty}(E)$ l'ensemble des fonctions boréliennes sur $E$ bornées m-presque sûrement, où $m$ est une mesure quasi-invariante quelconque sur $E$. 
Désignons par $m_{G}$ une mesure de Haar à droite sur $G$. Une mesure $\mu$ de $M^{1}(G)$ est dite étalée (voir [1]) si elle vérifie l'une des conditions équivalentes suivantes :

a) Il existe un entier $n$ tel que $\mu^{n}$ ne soit pas singulière par rapport à $m_{G}$. b) Il existe un entier $p$ tel que $\mu^{P}$ majore un multiple de $m_{G}$ sur un sous-ensemble ouvert non vide de $G$.

Nous notons alors $S_{\mu}$ l'ensemble des points $g$ de $G$ pour lesquels on peut trouver une mesure de Haar $m$ sur $G$ et un entier $p$ tels que la restriction de $m$ à un voisinage de $g$ soit majorée par ${ }_{\mu}{ }^{P} ; S_{\mu}$ est un semi-groupe ouvert non vide de $G([1])$.

Une mesure $\mu$ de $M^{1}(G)$ est dite apériodique si le sous-groupe fermé, $G_{\mu}$, engendré par le support de $\mu$ est le groupe $G$ entier.

6.3.-Nous appelons jauge ([12]) (resp. fonction sous additive) sur $G$, toute fonction borélienne positive $\delta$ sur $G$ telle que

$$
\forall(x, y) \in G \times G, \delta(x y) \leqslant \delta(x)+\delta(y)+C \quad(\operatorname{resp} . \delta(x y) \leqslant \delta(x)+\delta(y)),
$$

où C désigne une constante positive.

Si G est compactement engendré, une jauge $\delta$ est dite principale s'il existe un voisinage compact $V$ de $e$ engendrant $G$ tel que pour tout entier naturel $n, B_{n}=\{x: \delta(x) \leqslant n\} \subset v^{n}$. On sait que sur $G$ il existe de telles jauges (par exemple si $V$ est un voisinage compact de $e$ engendrant $G$, l'application $\rho$ définie par $\rho(x)=\inf \left\{n \in \mathbb{N}^{*}: x \in V^{n}\right\}$, en est une). Si $\delta_{0}$ est une jauge principale, pour toute autre jauge nous avons ([12]),

$$
\forall x \in G \quad \delta(x) \leqslant C_{1} \delta_{0}(x)+C_{1} .
$$

où $C_{1}$ est une constante positive.

Pour $\mu$ donnée dans $M^{1}(G)$ et $\alpha$ donné dans $\mathbb{R}_{+}$, la condition, $\int_{G}\left[\delta_{0}(x)\right]^{\alpha} \mu(d x)<+\infty$, est indépendante $d u$ choix de $\delta_{0}$; dans ce cas nous dirons que $\mu$ possède un moment d'ordre $\alpha$. .

En fait, en modifiant légèrement la démonstration de ([20]) on montre qu'étant donné un voisinage compact $V$ de e engendrant $G$, il existe des métriques (dites principales) invariantes à gauche (ou à droite, si l'on veut) telle que la boule de centre $e$ et de rayon $n$ soit contenue pour tout $n \geqslant 1$, dans $v^{n} ; s i$ $d$ est alors une telle distance, dire que $\mu$ possède un moment d'ordre $\alpha$ c'est dire que

$$
\int_{G}[d(e, x)]^{\alpha} \mu(d x)<+\infty .
$$


Fonctions harmoniques.-

6.4.-Soient $E$ un G-espace à gauche localement compact, f une fonction borélienne sur $E$ et $\lambda \in M^{1}(E)$. Nous notons $f * \lambda$ la fonction sur $G$ définie par

$$
f * \lambda(g)=\langle g \lambda, f\rangle=\int_{E} f(g \cdot x) \lambda(d x) \quad(g \in G),
$$

lorsque le second membre est défini pour tout $g \in G$.

Soit $\mu \in M^{1}(G)$. Nous appelons fonction $\mu$-hàrmonique bornée (sur $G$ ) toute fonction borélienne bornée $f$ sur $G$ telle que $f=f * \mu$, c'est-à-dire telle que

$$
\int_{G} f\left(g g^{\prime}\right) \mu\left(d g^{\prime}\right)=f(g) \quad(g \in G),
$$

Nous désignons par $E_{\mu}$ l'ensemble des fonctions $\mu$-harmoniques bornées (sur $G$ ) et par $H_{\mu}$ le sous ensemble de $E_{\mu}$ formé des fonctions uniformément continues à gauche (u.c.g.). Munis de la topologie de la convergence uniforme, $E_{\mu}$ et $H_{\mu}$ sont des espaces de Banach.

Soit $E$ un G-espace à gauche et $f$ une fonction sur $E$. Nous définissons $f^{g}: x \in E \longmapsto f(g \cdot x) \quad(g \in G, x \in E)$.

Ainsi, $G$ opère à droite sur $E_{\mu}$ et cette action induit sur $H_{\mu}$ une structure de G-espace à droite.

6.5.-Soient $H$ un sous groupe fermé de $G, H^{G}$ l'espace des classes modulo $H$ à gauche (i.e. $H^{G}=\{H g, g \in G\}$ ) et $p$ l'application naturelle de $G$ sur $H^{G}$. Soit $\mu \in M^{1}(G)$. Nous appelons fonction $\mu$-harmonique bornée sur ${ }_{H}{ }^{G}$ toute fonction borélienne bornée $f$ sur $H^{G}$ telle que la fonction fop soit $\mu$-harmonique bornée sur G.

Soit $H$ un espace de fonction sur $G$. Un élément $h$ de $G$ est appelé une période à gauche de $H$ si

$$
f(h g)=f(g) \quad(g \in G, f \in H) .
$$

L'ensemble des périodes à gauche de $H$ est évidemment un sous-groupe de $G$.

Soit $E_{\mu}^{H}=\left\{f \circ p, f \quad \mu\right.$-harmonique bornée sur $\left.H^{G}\right\}$. Un élément $\times$ de $J^{G}$ est appelé une $\mu$-période de $H^{l^{G}} s^{\prime i l}$ est l'image par $P$ d'une période à gauche de $E_{\mu}^{H}$. Autrement dit $x$ est une $\mu$-période de $\mathrm{H}^{G}$ si pour toute fonction $f$ $\mu$-harmonique bornée sur $H^{G}$ nous avons

$$
f(x \cdot g)=f(x) \quad(g \in G) .
$$


L'ensemble des $\mu$-périodes de $H^{V^{G}}$ est évidemment de la forme $H^{G^{\prime}}$, où G' est un sous-groupe de $G$ coritenant $H$.

(Noter que pour $H=\{e\}$, la notion de $\mu$-période de $G$ que l'on obtient est différente de celle de $[1]$ ).

Si $H^{\prime}$ est un sous-groupe fermé de $H$, l'image par l'application naturelle $s$ de $H^{l^{G}}$ sur $H^{I^{G}}$ d'une $\mu$-période de $H^{l^{G}}$ est une $\mu$-période de $H^{l^{G}}$. Si $f$ est $\mu$-harmonique sur $H^{\prime} l^{G}$ et tout élément de $H^{\prime} l^{H}$ est une $\mu$-période de $H^{\prime},^{G}$, il est clair que $f$ est la composée par $s$ d'une fonction $\mu$-harmonique sur $H^{G}$. Enfin si $H$ est distingué, f est $\mu$-harmonique sur. ${ }_{H}{ }^{G}$ signifie aussi que $f$ est $P(\mu)$-harmonique sur $H^{G}$.

Marches aléatoires et fonctions harmoniques.

6.6.-Soit $\Omega^{\prime}$ l'espace $G^{\mathbb{N}}$ muni de la plus petite tribu rendant mesurables les applications coordonnées de $\Omega^{\prime}$ dans $G$ (muni de la tribu borélienne). Soient $\mu \in M^{1}(G)$ et $Q$ la mesure de probabilité sur $\Omega^{\prime}$ produit des mesures $\mu$ sur chaque facteur. Soit $\Omega=G^{\mathbb{N}}$, muni de la tribu $\mathfrak{\xi}$, produit des tribus boréliennes sur chaque facteur. Pour tout $g \in G$ appelons $\mathbb{P}_{g}$ la mesure de probabilité sur $\Omega$, image de $\Omega$ par l'application de $\Omega^{\prime}$ dans $\Omega$ définie comme suit

$$
\left(g_{1}, g_{2}, \ldots\right) \longmapsto\left(g, g_{1}, g_{1} g_{2}, \ldots\right)
$$

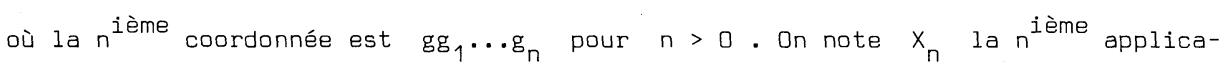
tion coordonnée de $\Omega$ sur $G$.

La marche aléatoire droite de loi $\mu$ sur $G$ est le quadruplet

$\left(\Omega, \mathcal{F},\left(X_{n}\right)_{n} \in \mathbb{N},\left(\mathbb{P}_{g}\right)_{g} \in G\right)$.

Soit $E$ un G-espace à droite. Nous appelons chaîne de loi $\mu$ sur $E$ la chaîne de Markov $\left\{\Omega, \mathscr{F},\left(u_{0} \cdot x_{n}\right)_{n \in \mathbb{N}},\left(\mathbb{P}_{g}\right)_{g} \in G\right\}$ (où $u_{0} \in E$ ) dont la probabilité de transition $Q$ est définie par

$$
Q(x, U)=\varepsilon_{x} * \mu(U)=\int_{G} 1_{U}(x \cdot g) \mu(d g) .
$$

On a une notion analogue pour un G-espace à gauche.

Nous allons à présent rappeler un résultat classique.

6.7.-Soit $\theta$ l'opérateur de translation sur $\Omega=G^{\mathbb{N}}$ définit par $\theta\left(\left\{x_{0}, \ldots, x_{n}, \ldots\right\}\right)=\left\{x_{1}, \ldots, x_{n}, \ldots\right\}$. Un événement $A$ de $\mathscr{f}$ est dit invariant si $\theta^{-1}(A)=A$. Les ensembles invariants forment une tribu et une variable aléatoire $Z$ est mesurable par rapport à cette tribu si et seulement si $Z o \theta=Z$. Une telle 
variable aléatoire est dite invariante. Deux ensembles invariants $A$ et $B$ sont dits équivalents si $\mathbb{P}_{g}[A \Delta B]=0, \forall g \in G$.

On sait alors que ([23], ch. 3, prop. (3.2)) :

Proposition. - La formule $h(x)=\mathbb{E}_{x}[Z]$ établit une correspondance biunivoque entre les classes d'équivalences de variables aléatoires invariantes et bornées $Z$, et les fonctions $\mu$-harmoniques bornées sur $G$. On a de plus

$$
\lim _{n \rightarrow+\infty} h\left(X_{n}\right)=Z \quad \mathbb{P}_{g} \text {-p.s. } \quad \forall g \in G .
$$

Nous utiliserons aussi le théorème suivant qui est une légère extension du théarème IV 1 de [1].

6.8. Théorème. - Soient $G$ un groupe L.C.D., $\mu$ une mesure de probabilité étalée sur $G, S_{\mu}$ le semi-groupe ouvert associé $\dot{a} \mu$ et $\left(\Omega, \mathcal{F},\left(X_{n}\right)_{n \in \mathbb{N}},\left(\mathbb{P}_{g}\right)_{g} \in G\right)$ la marche aléatoire droite de loi $\mu$ sur $G$. Soit $H$ un sous-groupe fermé de $G$. Soient $x$ et $g$ des éléments de G. Si pour $\mathbb{P}_{\mathrm{g}}$-presque tout $\omega$ de $\Omega$, il existe une suite $\left\{h_{n}(\omega)\right\}_{n \geqslant 1}$ d'éléments de $H$ telle que la suite $\left\{x_{n}^{-1}(\omega) h_{n}(\omega) \times x_{n}(\omega)\right\}_{n \geqslant 1}$ possède une valeur d'adhérence appartenant à $s_{\mu} s_{\mu}^{-1}$, alors pour toute fonction $f \mu$-harmonique bornée sur $H^{G}$ nous avons

$$
f \circ p(x g)=f \circ p(g)
$$

où $p$ désigne l'application naturelle de $G$ sur $H^{I^{G}}$.

Nous ne donnerons pas à cause de sa longueur la démonstration de ce théorème. Elle se calque aisément sur celle de [1], qui correspond au cas $H=\{e\}, g=e$. 
$G$ désigne un groupe de Lie ayant un nombre fini de composantes connexes. Nous reprenoris les notations des paragraphes précédents.

7.1. Lemme. - (voir [26]) Soit G un groupe de Lie ayant un nombre fini de composantes connexes, dont la composante connexe $G_{0}$ de l'unité est semi-simple. Soient $G=$ NAK une décomposition d'Iwasawa de $G$ (voir (1.8)) et $\mu$ une mesure de $M^{1}(G)$ étalée. Désignons par Q la probabilité de transition de la chấne de Zoi $\mu$ sur $K\left(\simeq N{ }_{N}^{G}\right)(v o i r(6.6))$. Alors pour tout $\varepsilon>0$, il existe un entier $n_{0}$ et un réel $\delta>0$ tels que pour tout borélien $\omega$ de $K$ vérifiant $m(W)<\delta$ on ait $Q^{n}(k, W)<\varepsilon$ pour tout entier $n \geqslant n_{0}$ et tout élément $k$ de $K$, où ${ }^{m} K$ désigne une mesure de Haar à gauche sur $K$.

Preuve. - Nous avons $G_{0}=N A K$ et $K=K L$, où $L$ est un sous-groupe compact de G (voir prop. (1.7)).

Si $H$ est un groupe localement compact, on désigne par $m_{H}$ une mesure de Haar à gauche sur $H$. Nous savons ([13]) que l'on $a$; moyennant des normalisations convenables,

$$
m_{G_{0}}=m_{N A} * m_{K} \text {. }
$$

Posons

$$
\begin{aligned}
& m_{N A}^{\prime}=\int_{L} \varepsilon_{\ell} * m_{N A} * \varepsilon_{\ell}{ }^{-1} m_{L}(d l) \\
& m_{K}^{\prime}=\int_{L} \varepsilon_{\ell} * m_{K} * \varepsilon_{\ell-1} m_{L}(d \ell) \\
& m_{K}=m_{K}^{\prime} * m_{L} .
\end{aligned}
$$

Il est facile de voir que $m_{N A}^{\prime}, m_{K}^{\prime}, m_{K}$ et $m_{G}=m_{N A}^{\prime} * m_{K}$ sont respectivement des mesures de Haar à gauche sur NA, $K, K$ et $G$.

Comme $\mu$ est étalée, on sait que pour $n$ suffisamment grand nous avons

$$
\mu^{n}=f_{n} \cdot m_{G}+\nu_{n} \text { avec } \quad\left\|\nu_{n}\right\|<\rho^{n} \quad(0<\rho<1)
$$

où $f_{n} \in L^{1}\left(m_{G}\right)$ et $\nu_{n}$ est une mesure étrangère à $m_{G}$.

Soit $\left(\varphi_{n, p}\right\}_{p \geqslant 1}$ une suite croissante de fonctions bornées et à support compact convergeant simplement yers $f_{n}$. D'après le théorème de convergence dominée, nous avons $f_{n}-\varphi_{n, p} \frac{L^{1}}{p \rightarrow+\infty} 0$ et par suite 


$$
\left\|f_{n} \cdot m_{G}-\varphi_{n, p} \cdot m_{G}\right\| \stackrel{p \rightarrow+\infty}{\longrightarrow} 0 \text {. }
$$

Soit $\varepsilon>0$. Choisissons $n$ assez grand pour que $\rho^{n}<\varepsilon / 3$; choisissons alors $p$ de façon que $\left\|f_{n} \cdot m_{G}-\varphi_{n, p} \cdot m_{G}\right\|<\varepsilon /_{3}$. Il vient

$$
Q^{n}(k, W) \leqslant 2 \varepsilon /_{3}+\int_{G} 1_{W}(\overline{k g}) \varphi_{n, p}(g) m_{G}(d g),
$$

où l'on désigne par $\bar{x}$ la composante sur $K$ de $x \in G$,

$$
\begin{aligned}
& \leqslant 2 \varepsilon / 3+\int_{G} 1_{W}(\bar{g}) \varphi_{n, p}\left(k^{-1} g\right) m_{G}(d g) \\
& \leqslant 2 \varepsilon / 3+\int_{N A} \int_{K} 1_{W}\left(k_{1}\right) \varphi_{n, p}\left(k^{-1} s k_{1}\right) m_{N A}^{\prime}(d s) m_{K}\left(d k_{1}\right) .
\end{aligned}
$$

Or $\varphi_{n, p}$ est une fonction bornée et à support compact ; désignons par $F$ le support de $\varphi_{n, p}$ et posons $b=\sup _{x \in G}\left|\varphi_{n, p}(x)\right|<+\infty$. F étant un compact de $G$, il existe des compacts $U$ et $V$ respectivement de $N A$ et $K$ tels que $F \subset U V$; il s'ensuit que pour $k \in K, k F \subset U^{\prime} K$, où $U^{\prime}=\bigcup_{k \in K} k \cup k^{-1}$ est un compact de NA (car Ad ef $K$ est compact).

Nous avons alors

$$
Q^{n}(k, W) \leqslant 2 \varepsilon / 3+b m_{N A}^{\prime}\left(U^{\prime}\right) m_{K}(W)
$$

et le lemme est prouvé.

Si dans le lemme (7.1) nous supposons que la mesure $m_{K}$ est finie (i.e. $K$ est compact), nous voyons que $Q$ vérifie la condition de Doeblin ([6]).

Nous avons alors :

7.2. Corollaire. - Avec les notations du Zemme (7.1), si $G_{0}$ est de plus de centre fini, la probabilité de transition Q vérifie la condition de Doeblin pour la mesure de Haar du groupe compact $K$.

Soient $G$ un groupe L.C.J. et $\mu$ une mesure de $M^{1}(G)$. Désignons par $\left(\Omega, \mathfrak{F},\left(X_{n}\right)_{n} \in \mathbb{N},{ }^{\left(P_{g}\right) g \in G}\right)^{\prime}$ la marche aléatoire droite de loi $\mu$ sur $G$. Un sous-groupe fermé $C$ de $G$ est dit récurrent si on a

$$
\mathbb{P}_{g}\left[\lim { }_{n} \sup \left\{x_{n} \in C\right\}\right]=1 \text { pour tout } g \in G \text {. }
$$

On considère alors le temps d'arret $S_{C}=\inf \left\{n>0: x_{n} \in C\right\}$, la probabilité de transition $P_{C}$ sur $G$ définie par

$$
P_{C}(g, A)=\mathbb{P}_{g}\left[X_{S} \in A\right] \quad(g \in G, A \text { borélien de } G)
$$


et la mesure $\mu^{C}$ de $M^{1}(C)$ définie par

$$
\mu^{C}(U)=\mathbb{P}_{e}\left[X_{S_{C}} \in U\right] \quad(U \text { borélien de } C) \text {. }
$$

Nous avons (avec les notations de $(6.4))$ :

7.3. Lemme. - ([22]) Soient $G$ un groupe L.C.D. et $\mu$ une mesure de $M^{1}(G)$. Soit C un sous-groupe récurrent de G. Alors l'application

$$
\begin{gathered}
j: E_{\mu}{ } \longrightarrow E_{\mu} \\
f \longmapsto \tilde{f}: g \longmapsto \tilde{f}(g)=P_{C} f(g)
\end{gathered}
$$

est une C-isométrie de $\mathrm{E}_{\mu}$ sur $\mathrm{E}_{\mu}$ (qui envoie $\mathrm{H}_{\mu}$, sur $\mathrm{H}_{\mu}$ quand $\mathrm{C}$ est ouvert). De plus, pour $\underset{f}{\sim} \underset{\mu}{\mu} E_{\mu}, j^{-1}(\tilde{f})$ est la restriction de $\tilde{f} \dot{a} C$.

Preuve. - Soit $\tilde{f}$ une fonction $\mu$-harmonique bornée sur G. D'après [23], nous savons que l'on a $P_{C} \tilde{f}=\tilde{f}$. Pour $h \in C$, il vient alors

$$
\begin{aligned}
\tilde{f}(h) & =P_{C} \tilde{f}(h)=\mathbb{E}_{h}\left[\tilde{f}\left(x_{S_{C}}\right)\right] \\
& =\mathbb{E}_{e}\left[\tilde{f}\left(h x_{S_{C}}\right)\right]=\int_{C} \tilde{f}(h x) \mu^{C}(d x) .
\end{aligned}
$$

Autrement dit la restriction de $\tilde{f}$ à $C$ est une fonction $\mu^{C}$-harmonique bornée sur C.

Inversement, soit $f \in E \quad$ (on a donc $P_{C} f(h)=f(h)$, pour tout $h \in C$ ) et considérons la fonction sur $G, \tilde{f}=P_{C} f$. ${ }^{C}$ est une fonction borélienne bornée. En outre nous avons Or

$$
\int_{G} \tilde{f}\left(g g^{\prime}\right) \mu\left(d g^{\prime}\right)=\mathbb{E}_{g}\left[\tilde{f}\left(x_{1}\right)\right]=\mathbb{E}_{g}\left[\tilde{f}\left(x_{1}\right) 1_{C}\left(x_{1}\right)\right]+\mathbb{E}_{g}\left[\tilde{f}\left(x_{1}\right){ }_{C}{ }^{c}\left(x_{1}\right)\right] .
$$

$$
\begin{aligned}
\tilde{f}\left(x_{1}\right) 1_{C}\left(x_{1}\right) & =f\left(x_{1}\right) 1_{C}\left(x_{1}\right) \quad \text { (puisque } f \text {. est } \mu^{C} \text {-harmonique) } \\
& =f\left(x_{S_{C}}\right) 1_{C}\left(x_{1}\right) .
\end{aligned}
$$

et

$$
\begin{aligned}
\mathbb{E}_{g}\left[\tilde{f}\left(x_{1}\right) 1_{C}\left(x_{1}\right)\right] & =\mathbb{E}_{g}\left[\mathbb{E}_{x_{1}}\left(f\left(x_{S_{C}}\right)\right) 1_{C} c^{c}\left(x_{1}\right)\right] \\
& =\mathbb{E}_{g}\left[\mathbb{E}_{g}\left[f\left(x_{S_{C}} a \theta\right) \mid \mathcal{F}_{1}\right] 1_{C}\left(x_{1}\right)\right]
\end{aligned}
$$

où $\mathfrak{F}_{1}$ est la tribu engendrée par $x_{1}$. (propriété de Markov) 


$$
\begin{aligned}
& =\mathbb{E}_{g}\left[f\left(x_{S_{C}} \circ \theta\right) 1_{C}{ }^{c}\left(x_{1}\right)\right] \\
& =\mathbb{E}_{g}\left[f\left(x_{S_{C}}\right) 1_{C}\left(x_{1}\right)\right] .
\end{aligned}
$$

D' où

$$
\int_{G} \tilde{f}\left(g g^{\prime}\right) \mu\left(d g^{\prime}\right)=\mathbb{E}_{g}\left[f\left(x_{S_{C}}\right)\right]=\tilde{f}(g) \text {. }
$$

Autrement dit $\tilde{f}$ appartient à $E_{\mu}$.

D'autre part soient $f \in E_{\mu^{C}}$ et $h \in C$; nous avons

$$
\widetilde{f^{h}}(g)=\mathbb{E}_{g}\left[f^{h}\left(x_{S_{C}}\right)\right]=\mathbb{E}_{g}\left[f\left(h x_{S_{C}}\right)\right]=\mathbb{E}_{h g}\left[f\left(x_{S_{C}}\right)\right]=\tilde{f}^{h}(g)
$$

ce qui montre que $j$ commute avec l'action de $C$.

Enfin, si $C$ est ouvert dans $G$, les relations

$$
\left\|j(f)^{h}-j(f)\right\|_{\infty}=\left\|j\left(f^{h}\right)-j(f)\right\|_{\infty}=\left\|f^{h}-f\right\|_{\infty}, \quad f \in E_{\mu}, h \in c,
$$

montrent que $f$ est uniformément continue à gauche si et seulement si $j(f)$ l'est. Ce qui achève la démonstration du lemme (7.3).

7.4. Corollaire. - Soient G un groupe L.C.D. et $\mu$ une mesure de $M^{1}(G)$. Soient C un sous-groupe récurrent de $G$ et $E$ un G-espace localement compact (à gauche ou à droite) alors toute mesure $\nu$ de $M^{1}(E) \mu$-invariante est $\mu^{C}$-invariante.

Preuve. - Nous nous bornons à faire la démonstration pour un G-espace à gauche. Soit $v$ une mesure de $M^{1}(E)$. Désignons par $C_{K}(E)$ l'espace des fonctions continues à support compact sur $E$. A tout $f \in C_{K}(E)$ associons la fonction $\psi_{\nu}(f)$ définie par

$$
\psi_{\nu}(f): g \in G \longmapsto \int_{E} f(g \cdot u) v(d u) ;
$$

il est facile de voir que $\psi_{v}(f)$ est bornée et U.C.G. . Nous notons $\left.\psi_{v}(f)\right|_{C}$ sa restriction à $c$.

Avec ces notations nous avons alors :

$\nu$ est $\mu$-invariante $\Longleftrightarrow$ i) $\psi_{\nu}(f) \in H_{\mu}$, pour tout $f$ de $C_{K}(E)$

$\nu$ est $\mu^{C}$-invariante $\Leftrightarrow$ ii) $\left.\psi_{\nu}(f)\right|_{C} \in \mathrm{H}_{\mu}$, pour tout $f$ de $C_{K}(E)$.

D'après le lemme (7.3), nous avons i) $\Longrightarrow$ ii); ce qui établit le corollaire.

7.5. Lemme. - Soient G un groupe de Lie ayant un nombre fini de composantes connexes et $\mu$ une mesure de $M^{1}(G)$ étalée. Alors il existe sur la frontière maximale $\mathrm{B}(\mathrm{G})$ de $\mathrm{G}$ une unique mesure de probabilité $\mu$-invariante (i.e. telle que $v * \mu=v$ ). 
Preuve. - Notons d'abord que puisque $B(G)$ est compact, il existe toujours sur $B(G)$ une mesure de probabilité $\mu$-invariante.

Le sous-groupe fermé, $G_{\mu}$, engendré par le support de $\mu$ contient la composante connexe $G_{0}$ de l'unité dans $G$. $B(G)$ est aussi une frontière maximale de $G_{\mu}$ (lemme (2.2)). Nous pouvons donc, quitte à remplacer $G$ par $G_{\mu}$, supposer que $\mu$ est apériodique.

Ecrivons $B(G)=H(G) l^{G}$; il revient alors au même de montrer que sur $B^{\prime}=G / H(G)$ il existe une unique mesure $\mu$-invariante $\nu \in M^{1}\left(B^{\prime}\right)$ (i.e. telle que $\mu * \nu=\nu$ ). Nous allons prouver que $l$ 'on peut se ramener au cas où $G$ est un groupe de Lie semi-simple connexe de centre fini, le lemme résultera alors de la proposition II.4 de [1].

$G_{0} \backslash^{G}$ est fini; comme $\mu$ est apériodique, $G_{0}$ est un sous-groupe récurrent de $G$ (i.e. $\mathbb{P}_{g}\left[\lim \sup \left\{X_{n} \in G_{0}\right\}\right]=1$, pour tout $g$ de $\left.G\right)$. D'après le corollaire (7.4), si $v$ est une mesure, $\mu$-invariante, de $M^{1}\left(B^{\prime}\right), \nu$ est aussi $\mu^{G_{0}}$-invariante. La mesure $\mu^{G_{0}}$ de $M^{1}\left(G_{0}\right)$ étant étalée, on est ramené à prouver le lemme pour un groupe de Lie connexe.

Soit alors $R^{\prime}$ le plus grand sous-groupe distingué résoluble de $G ; R^{\prime} l^{G}$ est un groupe de Lie semi-simple connexe de centre trivial. B' est une frontière maximale (gauche) de $R^{\prime} l^{G}$. Désignons par $\xi^{\prime} l^{\prime}$ application naturelle de $G$ sur $R^{\prime} l^{G}$; si $\nu \in M^{1}\left(B^{\prime}\right)$ est $\mu$-invariante, elle est aussi $\xi^{\prime}(\mu)$-invariante. $\mu$ étant étalée, la mesure $\xi^{\prime}(\mu)$ de $M^{1}\left(R_{R}, l^{G}\right)$ est étalée ([1] lemme IV.9). On est alors ramené à prouver le lemme dans le cas où $G$ est un groupe de Lie semi-simple connexe de centre trivial. Le lemme est donc prouvé.

7.6. Corollaire. - Soient G un groupe de Lie ayant un nombre fini de composantes connexes et $\mu$ une mesure de $M^{1}(G)$ étalée. Alors la châ̂ne de loi $\mu$ sur la frontière maximale $\mathrm{B}(\mathrm{G})$ de $\mathrm{G}$ est ergodique (i.e. possède une seule classe ergodique sans classe cyclique, voir [6], ch. 5, \$5).

Preuve. - La chaine de loi $\mu$ sur B(G) est aussi la chaine de loi $\xi^{\prime}(\mu)$ sur $\overline{B(G)}$ où $\xi^{\prime}$ désigne l'application naturelle de $G$ sur $R^{\prime} I^{G}$. D'après le corollaire (7.2) cette chaine vérifie la condition de Doeblin. En raison de l'unicité de la mesure $\mu$-invariante sur $B(G)$ (lemme 7.5) il existe une seule classe ergodique. Cette classe ne peut pas contenir de sous classe cyclique ; car une telle sous classe deviendrait une classe ergodique pour la chaine de loi $\mu^{d}$, où d désigne la période de cette sous classe; ce qui contredirait le fait que $\mu^{d}$ possède une unique mesure invariante sur $B(G)$. (Voir [26], corollaire du lemme 1). 
7.7. Lemme. - Soient G un groupe de Lie ayant un nombre fini de composantes connexes; $\mu$ une mesure de $M^{1}(G)$ apériodique et étalée; et $G_{1}$ un sous-groupe (fermé) de $G$ contenant $G_{0}$.

Alors il existe sur $\mathrm{B}(\mathrm{G}) \times{ }_{G_{1}}{ }^{G}$ une unique mesure de probabilité $\mu$-invariante qui est la mesure produit $\nu \otimes m$, où $v$ est l'unique mesure $\mu$-invariante de $M^{1}(B(G))$ (Zemme (7.5)) et $m$ la mesure de Haar normalisée sur $G_{1}{ }^{G}$. La chaine de loi $\mu$ sur $B(G) \times G_{1} l^{G}$ est done ergodique.

Preuve. - Comme pour le lemme (7.5) nous raisonnons sur les G-espaces à gauche. On est donc amené à montrer qu'il existe sur le G-espace homogène produit $B^{\prime} \times G / G_{1}$ (où $B^{\prime}=G / H(G)$ ), une unique mesure de probabilité $\mu$-invariante. $\mu$ étant supposée apériodique, la.composante connexe $G_{0}$ de l'unité dans G est un sous-groupe récurrent de $G$. Si $v_{1}$ est une mesure $\mu$-invariante de $M^{1}\left(B^{\prime} \times G / G_{1}\right)$, d'après le corollaire $(7.4), \nu_{1}$ est aussi $\mu{ }^{G}$-invariante; nous avons donc

$$
\begin{aligned}
\left(\mu^{G}\right)^{n} * v_{1}(V \times W) & =\int_{G_{0}} \int_{B^{\prime} \times x^{G} / G_{1}} 1_{V}(g \cdot x) 1_{W}(y)\left(\mu^{G^{0}}\right)^{n}(d g) v_{1}(d x, d y) \\
& =\int_{B^{\prime} \times x^{G} / G_{1}} 1_{W}(y)\left(\int_{G_{0}} 1_{V}(g \cdot x)\left(\mu^{G}\right)^{n}(d g)\right) v_{1}(d x, d y) \\
& =v_{1}(V \times W),
\end{aligned}
$$

pour tout borélien $V$ (resp. W) de $B^{\prime}$ (resp. de $G / G_{1}$ ) et tout entier $n$. Mais d'après le corollaire (7.6) la chaine de loi $\mu^{{ }}{ }$sur $B^{1}$ est ergodique ; nous avons alors $([6]$, ch. $5, \$ 5)$,

$$
\sup _{b \in B^{\prime}} \sup _{V}\left|\left(\mu^{G^{\circ}}\right)^{n} * \varepsilon_{b}(V)-v(V)\right|<\alpha e^{-\beta n}
$$

où $\alpha$ et $\beta$ sont des réels $>0$ et $\nu$ est l'urique mesure $\mu^{G}$-invariante de $M^{1}\left(B^{\prime}\right)$.

Il s'ensuit alors que :

$$
\left(\mu^{G_{0}}\right)^{n} * v_{1}(V \times M) \longrightarrow v(V) v_{1}\left(B^{\prime} \times W\right) \text {. }
$$

Comme il est facile de voir que la projection de $\nu_{1}$ sur $G / G_{1}$ est nécessairement la mesure de Haar normalisée sur $G /_{G_{1}}$, il s'ensuit que $\nu_{1}=\nu \otimes m$. D'après le lemme (7.5) et le corollaire (7.4) v est aussi l'unique mesure $\mu$-invariante de $M^{1}\left(B^{\prime}\right)$. La première assertion du lemme (7.7) est donc prouvée. 
Pour prouver que la chaine de loi $\mu$ sur $B(G) \times G_{1} \backslash^{G}$ est ergodique, il suffit de reprendre la démonstration du corollaire (7.6). Le lemme (7.7) est prouvé.

7.8. Proposition. - Soient $G_{1}$ un sous-groupe de $G$ contenant $G_{0}$ et $\rho$ un A-cocycle K-invariant sur le G-espace homogène à droite $\mathrm{B}(\mathrm{G}) \times{ }_{\mathrm{G}_{1}}{ }^{\mathrm{G}}$ (voir définition (2.1)). Soit $\mu$ une mesure de $M^{1}(G)$ apériodique et désignons par $\left(\Omega, \mathcal{F},\left(x_{n}\right)_{n \in \mathbb{N}},\left(\mathbb{P}_{g}\right)_{g} \in G\right)$ Za marche aléatoire droite de loi $\mu$ sur $G$.

Alors si o n'est pas nul, nous avons l'alternative suivante: ou bien, pour tout $u$ de $\mathrm{B}(\mathrm{G}) \times \mathrm{G}_{1} \backslash \mathrm{G}, \rho\left(\mathrm{u}, \mathrm{x}_{n}\right)$ converge vers $(-\infty), \mathbb{P}$-p.s. oubien, pour tout $u$ de $B(G) \times G_{1} \backslash G, \rho\left(u, X_{n}\right)$ converge vers $(+\infty), \mathbb{P}{ }_{g}-p \cdot s$. oubien, pour tout $u$ de $B(G) \times G_{1} \backslash G, \lim _{n} \sup \rho\left(u, X_{n}\right)=+\infty$ et $\lim _{n}$ inf $\rho\left(u, x_{n}\right)=-\infty \quad \mathbb{P}_{g}$-p.s. $\forall g \in G$.

Preuve. - Désignons par $\zeta_{1}$ l'application naturelle de $G$ sur $G_{1} \backslash^{G}$; par $U_{0}$ l'élément de $B(G)$ stable par $N(D)$, (lemme (2.3)) et par vo l'álément $\left(u_{0}, \zeta_{1}(e)\right)$ de $B(G) \times G_{1} \backslash^{G}$. Pour tout réel $\delta$, posons $f_{\delta}(g)=\mathbb{P}_{g}\left[\lim { }_{n} \sup \rho\left(v_{0}, x_{n}\right)>\delta\right]=\mathbb{P}_{e}\left[\lim _{n} \sup \rho\left(v_{0}, g x_{n}\right)>\delta\right] \quad(g \in G) ;$ $f_{\delta}$ est une fonction $\mu$-harmonique bornée (voir (6.7)). Notons $[D, D]$ le sous-groupe de $D$ engendré par les commutateurs $x \mathrm{y}^{-1} \mathrm{y}^{-1}$, $x$ et $y \in D$. $[D, D] \Gamma$ est un sous-groupe de $G$ contenu dans $N_{0}(D)$. Pour $h \in[D, D] \Gamma$ et $y \in G$ nous avons :

$$
\begin{aligned}
& \rho\left(v_{0}, h y\right)=\rho\left(v_{0}, h\right)+\rho\left(v_{0}, h, y\right)=\rho\left(v_{0}, h\right)+\rho\left(v_{0}, y\right) \\
& \text { or si } h=x \gamma \text { avec } x \in[D, D] \text { et } \gamma \in \Gamma \text { il vient } \\
& \rho\left(v_{0}, h\right)=\rho\left(v_{0}, x\right)=\phi(x)=0
\end{aligned}
$$

où $\phi$ désigne l'homomorphisme de $D$ dans $\mathbb{R}$ additif associé à $\rho$ (lemme (2.4)) d' où

$$
\rho\left(v_{0}, h y\right)=\rho\left(v_{0}, y\right) \quad(h \in[0,0] \Gamma, g \in G) .
$$

Il s'ensuit alors que pour tout réel $\delta$ la fonction $\mu$-harmonique bornée $f_{\delta}$ vérifie

$$
f_{\delta}(h g)=f_{\delta}(g) \quad(h \in[D, D] \Gamma, g \in G) .
$$

Admettons un instant qu'une telle fonction $\mu$-harmonique bornée sur $G$ soit constante. D'après la proposition (6.7) nous avons alors $f_{\delta}=0$ ou $f_{\delta}=1$ pour 
tout réel $\delta$, c'est-à-dire que l'on a

ou bien $\rho\left(v_{0}, x_{n}\right)$ converge vers $(-\infty), \mathbb{P}_{g}-$ p.s. $\forall g \in G$

ou bien il existe $\delta_{0} \in \mathbb{R}$ tel que $\lim _{n} \sup \rho\left(v_{0}, x_{n}\right)>\delta_{0}, \mathbb{P}_{g}-p . s . \quad \forall g \in G$.

Dans le second cas, de $\rho\left(v_{0}, x_{n}\right)=\rho\left(v_{0}, d x_{n}\right)-\rho\left(v_{0}, d\right)(d \in D)$, il résulte que l'on a pour tout d de $D$

$$
\lim _{n} \sup \rho\left(v_{0}, x_{n}\right)>\delta_{0}-\rho\left(v_{0}, d\right)=\delta_{0}-\phi(d), \mathbb{P}_{g}-p \cdot s . \quad \forall g \in G .
$$

Mais $\rho$ étant non nul, l'homomorphisme $\phi$ de $D$ dans $\mathbb{R}$ additif qui lui est associé est non nul. D étant connexe, il s'ensuit donc que $\phi(D)=\mathbb{R}$; et par suite

$$
\lim _{n} \sup \rho\left(v_{0}, x_{n}\right)=+\infty \quad \mathbb{P}_{g}-p . s . \quad \forall g \in G .
$$

Enfin l'égalité

$$
\rho\left(v_{0}, k X_{n}\right)=\rho\left(v_{0} \cdot k, x_{n}\right) \quad(k \in k)
$$

montre qu'on est dans le même cas pour tous les éléments $u$ de $B(G) \times G_{1} \backslash G^{\prime}$.

En recommençant alors le raisonnement avec les fonctions

$$
f_{\delta}^{\prime}(g)=\mathbb{P}_{g}\left[\lim _{n} \text { inf } \rho\left(v_{0}, x_{n}\right)<\delta\right] \quad(\delta \in \mathbb{R}, g \in G)
$$

on obtient la proposition (7.8).

Cette proposition résulte donc des résultats:

7.9. Lemme. - Soit $G$ un groupe de Lie ayant un nombre fini de composantes connexes et $\mu$ une mesure de $M^{1}(G)$ apériodique et étalée. Alors toute fonction $\mu$-harmonique bornée $f$ sur $G$ telle que $f(h g)=f(g)(h \in[D, D], g \in G)$ est la composée par l'application naturelie de $G$ sur $D^{G}$ d'une fonction u-harmonique bornée sur $\backslash^{G}$.

Preuve. - $\mu$ étant étalée, on sait ([1], prop. 1.6) que $f$ est continue. II s'ensuit que $l$ 'on a

$$
f(h g)=f(g) \quad(h \in[\overline{0,0}] \quad g \in G) .
$$

Autrement dit $f$ est la composée par l'application naturelle de $G$ sur $\frac{}{[D, D]} \backslash G$
d'une fonction $\mu$-harmonique bornée $\bar{f}$ sur $\overline{[0,0]} \backslash^{G}$.

Appelons $P$ l'application naturelle de $G$ sur $\backslash^{G}$ et considérons une section mesurable $s$ de $P$ à valeurs dans $K$. Tout $g \in G$ s'écrit alors

$$
g=d(g)(\operatorname{sop}(g))
$$

avec $d(g)=g(\operatorname{sop}(g))^{-1} \in D$ et $\operatorname{sop}(g) \in K$.

Soit $\left(\Omega, \mathcal{F},\left(X_{n}\right)_{n \in \mathbb{N}},\left(\mathbb{P}_{g}\right)_{g} \in G\right)$ la marche aléatoire droite de loi $u$ sur 
G. Ecrivons $x_{n}=D_{n} k_{n}$ avec $D_{n}=d\left(x_{n}\right)$ et $k_{n}=\operatorname{sop}\left(x_{n}\right)$. Posons

Nous avons

$$
u_{n}=D_{n} d D_{n}^{-1} d^{-1} \in[D, D] \text {. }
$$

$$
x_{n}^{-1} U_{n} d x_{n}=k_{n}^{-1} d k_{n} \subset \bigcup_{k \in k} k^{-1} d k, \mathbb{P}_{g}-p . s . \quad \forall g \in G .
$$

Désignons par $S_{\mu}$ le semi-groupe ouvert associé à $\mu \cdot S_{\mu} S_{\mu}^{-1}$ est un ouvert de $G$ contenant $e$. Soit $V$ un voisinage compact de e contenu dans $S_{\mu} S_{\mu}^{-1}$. Comme Ad $_{y} K$ est. compact, $w=\bigcap_{k \in K} k^{-1} V k$ est un vọisinage ouvert de $e$ dans $G$. Pour tout $d$ appartenant à $D \cap W$ nous avons alors

$$
x_{n}^{-1} U_{n} d x_{n} \subset \bigcup_{k \in K} k^{-1} W k \subset v \subset s_{\mu} s_{\mu}^{-1}, \mathbb{P}_{g}-p \cdot s . \quad \forall g \in G .
$$

D'après le théorème (6.8) les éléments de $W \cap \square$ sont des $\mu$-périodes sur $\overline{[\mathrm{D}, \mathrm{D}]} \backslash^{G}$. D étant connexe, il s'ensuit que tous les éléments de $\mathrm{D}$ sont des H-périodes sur $\left.\frac{}{[D, D]}\right|^{G}$. Le lemme (7.9) est prouvé.

7.10. Corollaire. - Avec les notations du lemme (7.9), toute fonction $f \quad \mu$-harmonique bornée sur $G$ telze que $f(h g)=f(g)(h \in[D, D] \Gamma, g \in G)$ est constante.

Preuve. - D'après le lemme (7.9), f est la composée, par l'application naturelle de $G$ sur $N_{0}(D) \backslash^{G}$, d'une fontion $\hat{f} \mu$-harmonique bornée sur $N_{0}(D) \backslash^{G}$. Or $N_{0}(D) \backslash^{G}$ est $G$-homéomorphe au G-espace produit $B(G) \times G_{0} \backslash G$. D'après le lemme (7.7), il s'ensuit que la chaine de loi $\mu$ sur $\left.N_{0}(D)\right)^{G}$ est ergodique et est donc, en particulier, une chaine récurrente au sens de Harris. On sait alors que $\hat{f}$ et par suite $f$ sont constantes (voir [23]).

Remarque. - Dans la démonstration du lemme (7.9), la condition "apériodique" a été nécessaire pour savoir que la chaine de loi $\mu$ sur $B(G) \times{ }_{G} l^{G}$ est ergodique. Mais on sait (corollaire (7.6)) que la chaine de loi $\mu$ sur $^{\circ} B(G)$ est ergodique sans cette condition. Il s'ensuit alors que si $\mu$ est étalée, toute fonction $\mu$-harmonique bornée sur G telle que

$$
f(h g)=f(g) \quad\left(h \in[D, D] \Gamma^{\prime}, g \in G\right)
$$

est constante.

Il est alors facile de voir que pour un A-cocycle $\rho$ sur $B(G)$, la proposition (7.8) reste valable sans la condition d'apériodicité. 
7.11. Définition. - Soient $G$ un groupe L.C.D. tel que $G_{0}{ }^{G}$ soit compact et $\mu$ une mesure de $M^{1}(G)$. Soient $E$ un G-espace compact à droite et $\rho$ un $A$-cocycle sur $E$ (définition (2.1)). Nous disons que $\rho$ est $\mu$-négatif strict si pour tout $u$ de $E^{\prime}, \rho\left(u, X_{n}\right)$ converge vers $(-\infty), \mathbb{P}_{x}-$ p.s. $\forall x \in G$. Dans le cas contraire $\rho$ est dit $\mu$-positif.

7.12. Proposition. - Soit (G,E,p] comme dans la définition (7.11). Supposons que $\mu$ possède un moment d'ordre 1 et que la chaine de loi $\mu$ sur $E$ soit ergodique (i.e. qu'elle vérifie la condition de Doeblin et possède une seule classe ergodique sans classe cyclique, voir [6]) ; désignons par v l'unique mesure de probabilité $\mu$-invariante sur $E$ et posons $a_{\rho}=\int_{G} \int_{E} \rho(u, g) v(d u) \mu(d g)$.

Alors nous avons :

$a_{\rho}<0($ resp. $>0) \Longleftrightarrow \lim _{n} \rho\left(u, x_{n}\right)=-\infty$ (resp. $\left.+\infty\right), \mathbb{P}_{x}-$ p.s. $\forall x \in G$.

$$
\begin{array}{r}
a_{\rho}=0 \Longleftrightarrow \lim _{n} \inf \rho\left(u, x_{n}\right)=-\infty \text { et } \lim _{n} \sup \rho\left(u, x_{n}\right)=+\infty, \\
\mathbb{P}_{x}-p \cdot s . \\
\forall x \in G .
\end{array}
$$

Preuve. - La chaine de.loi $\mu$ sur $E$ étant ergodique, nous savons ([6]) que $\frac{1}{n} \rho\left(u, x_{n}\right)$ converge, $\mathbb{P}_{x}$-p.s. $\forall x \in G$, vers $a_{\rho}$. Le seule cas non trivial est alors celui où $a_{\rho}=0$.

Ecrivons $X_{n}=X_{0} Y_{1} \ldots Y_{n}$, où les $Y_{i}$ sont des variables aléatoires indépendantes et de loi $\mu$. Pour tout $u \in E$, le couple (u. $X_{n}, \rho\left(u \cdot X_{n-1}\right.$, $\left.Y_{n}\right)$ ) est une chaine de Markov homogène dont la probabilité de transition $\ngtr$ est définie par $\tilde{P} f(u, \alpha)=\int_{G} f(u \cdot g, \rho(u, g)) \mu(d g) \quad(u \in E, \alpha \in \mathbb{R}, f$ borélienne bornée Nous avoins alors $\tilde{P} f(u, \alpha)=\tilde{P} f(u, 0)$ et la chaine considérée est donc semi-markovienne (voir [16]). Ayant remarqué que

$$
\left(u \cdot x_{n}, p\left(u, x_{n}\right)\right)=\left(u \cdot x_{n}, \rho\left(u, x_{0}\right)+\sum_{p=1}^{n} \rho\left(u \cdot x_{p-1}, y_{p}\right)\right),
$$

la proposition (7.12) résulte alors du corollaire de la proposition 12 de. [16].

7.13. Proposition. - Soit $G$ un groupe de Lie ayant un nombre fini de composantes connexes dont la composante connexe de l'unité dans $G$ est semi-simple et de centre fini. Alors toute famille discriminante d'A-cocycles associée à $G$ (voir $\$ 2)$ est une famille d'A-cocycles $\mu$-négatifs stricts pour toute mesure $\mu$ de $M^{1}(G)$ étalée.

Preuve. - Nous reprenons les notations des paragraphes précédents. Considérons la

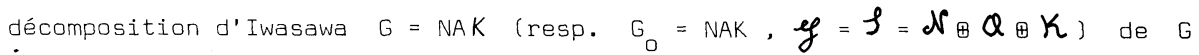


(resp. de $G_{0}, \boldsymbol{g}$ ). $\Xi$ est la famille des poids de la représentation adjointe de $\mathbb{P} \otimes \boldsymbol{Q}$ dans $\mathcal{P}$. Tout élément de $\Xi$ est nul sur $\mathcal{P}$ et sa restriction à $\boldsymbol{Q}$ est un poids de la représentation adjointe de $\boldsymbol{l}$ dans $\mathcal{N}$ (i.e. un élément de $\Sigma_{1}$ $(1.2))$. Nous identifions $\Xi$ et $\Sigma_{1}$.

Posons

$$
\left(\Sigma_{1}\right)_{-}=\left\{\lambda \in \Sigma_{1}: \rho_{\lambda} \text { est } \mu \text {-négatif strict }\right\}
$$

et

$$
\left(\Sigma_{1}\right)_{+}=\left\{\lambda \in \Sigma_{1}: \rho_{\lambda} \text { est } \mu \text {-positif }\right\} \text {. }
$$

$\left\{\left(\Sigma_{1}\right)_{-},\left(\Sigma_{1}\right\}_{+}\right\}$est une partition stable par addition et conjuguaison de $\Sigma_{1}$;

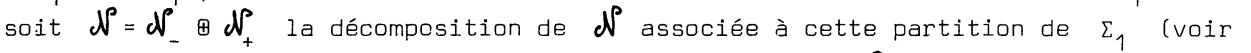
(3.7)). Muni du produit $x$ défini par la formule (3.3), $\mathcal{N}$ est le produit amalgamé des sous algèbres $\mathbb{P}_{-}$et $\mathcal{N}_{+}$. Comme $N$ est nilpotent simplement connexe, il s'ensuit que $N$ est le produit amalgamé des sous-groupes fermés $N_{-}=$(exp $\left.\mathcal{P}_{-}\right)$et $N_{+}=\left(\exp \mathbb{P}_{+}\right)$.

Nous avons alors le lemme :

Lemme. - Pour toute mesure $\mu$ étalée et apériodique, les fonctions $\mu$-harmoniques bornées sur $N_{-} \Gamma^{\prime} I^{G}$ sont constantes. ( $\Gamma^{\prime}$ désigne le sous-groupe de $G$ formé des éléments qui nörmalisent à la fois $\mathrm{N}$, A et $\mathrm{K}$, voir prop. (1.7)).

Preuve du lemme. - Par une méthode identique à celle que nous utiliserons pour démontrer la proposition (10.1), on montre que les fonctions $\mu$-harmoniques bornées sur $N_{-} \backslash^{G}$ sont les composées par 1'application naturelle de $N_{-}{ }^{G}$ sur $N^{G}$ des fonctions $\mu$-harmoniques bornées sur $N{ }^{G}$.

Le lemme est alors une conséquence de la remarque qui suit le corollaire (7.10).

Ceci dit, considérons la décomposition de Bruhat $G=N A \tilde{N} \Gamma$ ' mod. $m_{G}$, de $G$ (voir proposition (5.3)) et désignons par B l'application naturelle de $G$ sur la frontière maximale (droite) $F={ }^{G} /{ }_{A N \Gamma}$, de $G$. D'après la proposition (5.3) l'image de $N$ par $B$ est un ouvert de $F$ dont le complémentaire est de mesure nulle pour une quelconque mesure quasi-invariante sur $F$.

Désignons par $v$ l'unique mesure $\mu$-invariante de $M^{1}(F)$. $\mu$ étant étalée, on sait $([1]$ ) que $\nu$ est la restriction à un ouvert de $F$ d'une mesure quasi-invariante; $B(N)$ est donc de $v$-mesure égale à 1 .

L'applicatiion $N_{-} \times N_{+} \longrightarrow N$ est un isomorphisme de variétés analytiques. $\left(n_{-}, n_{+}\right) \longmapsto n_{-} n_{+}$

Désignons par $E_{K}\left(N_{+}\right)$l'espace des fonctions continues à support compact sur $N_{+}$ 
et par $m_{\Gamma}$, la mesure de Haar normalisée sur $\Gamma^{\prime}$ (noter que puisque $G_{0}$ est supposé être de centre fini, $\Gamma^{\prime}$ est un sous-groupe compact de G) . Considérons la fonction borélienne bornée sur $F$ définie par

$$
\begin{aligned}
f\left(\beta\left(n_{-} n_{+}\right)\right] & =\int_{\Gamma^{\prime}} \phi\left(\gamma n_{+} \gamma^{-1}\right) m_{\Gamma^{\prime}},(d \gamma) \text { où } \phi \in C_{K}\left(N_{+}\right) \\
f(u) & =0 \text { si } u \in F, u \notin \beta(N) .
\end{aligned}
$$

Pour tout $u \in \beta(N)$ et tout $h \in N_{-} \Gamma^{\prime}$, nous avons $f(h . u)=f(u)$ : en effet écrivons $u=\beta\left(n_{-} n_{+}\right)$et $h=n_{-}^{\prime} \gamma^{\prime}$ avec $n_{-}, n_{-}^{\prime} \in N_{-}, n_{+} \in N_{+}$et $\gamma^{\prime} \in \Gamma^{\prime}$; il vient

$$
\begin{aligned}
f(h . u) & =f \circ \beta\left(n_{-}^{\prime} \gamma^{\prime} n_{-} n_{+}\right)=f \circ \beta\left(n_{-}^{\prime}\left(\gamma^{\prime} n_{-} \gamma^{\prime-1}\right)\left(\gamma^{\prime} n_{+} \gamma^{\prime-1}\right)\right) \\
& =\int_{\Gamma^{\prime}} \phi\left(\gamma \gamma^{\prime} n_{+} \gamma^{\prime-1} \gamma^{-1}\right) m_{\Gamma^{\prime}}(d \gamma)=\int_{\Gamma^{\prime}} \phi\left(\gamma n_{+} \gamma^{-1}\right) m_{\Gamma^{\prime}}(d \gamma) \\
& =f(u) .
\end{aligned}
$$

La fonction $f * v$ qui à $g \in G$ associe $\int_{F} f(g . u) \nu(d u)$ est alors une fonction $\mu$-harmoriqque bornée telle que $f * v(h g)=f * v(g), h \in N_{-} \Gamma^{\prime}$ et $g \in G_{0}$. D'après ce qui précède, $f * v$ est donc constante. Or on sait que l'on peut trouver une suite $a_{n}$ d'éléments de $A$ telle que la suite $a_{n}^{-1} \times a_{n}$ converge vers e pour tout élément $x$ de $N$. (Il suffit de choisir un élément $H$ dans la chambre de Weyl $W$ (voir $\& 1$ ) et de poser. $\left.a_{n}=\exp (n H)\right)$. D'après le théorème de convergence dominée, il résulte alors que $f * v\left(g a_{n}^{-1}\right)$ converge vers foß $(g)$, pour tout $g$ appartenant à $G_{0}$. $f * \nu$ étant constante, on en déduit que foß est constante pour toute fonction $\phi$ de $C_{K}\left(N_{+}\right)$. On a alors nécessairement $N_{+}=\{e\}$, c'està-dire $\Sigma_{1}=\left(\Sigma_{1}\right)_{-}$. La proposition est démontrée.

7.14. Corollaire. - Avec les notations de la proposition (7.13), supposons que $\mu$ possède en outre un moment d'ordre 1, alors nous avons

$$
a_{\lambda}=\iint \rho_{\lambda}(u, g) v(d u) \mu(d g)<0, \quad(\lambda \in \Xi),
$$

où $v$ désigne l'unique mesure $\mu$-invariante de $M^{1}(B(G))$ (Zemme (?.5)).

Preuve. - Compte-tenu du corollaire (7.6) et de la proposition (7.12), le corollaire résulte immédiatement de la proposition (7.13).

7.15. Corollaire. - Soit G un groupe de Lie ayant un nombre fini de composantes connexes. Alors les éléments du sous ensemble $\left\{\rho_{\lambda}, \lambda \in \Sigma_{1}\right\}$ (voir (5.5)) de la famille discriminante d'A-cocycles $\left\{\rho_{\lambda}, \lambda \in \Xi\right\}$ associée d $G$ (voir \& 2) sont $\mu$-négatifs stricts, pour toute mesure $\mu$ de $M^{1}(G)$ étalée. 
Preuve. - Nous savons que $B(G)$ est une frontière maximale du groupe de $L i e R^{\prime} \backslash^{G}$ dont la composante connexe est semi-simple et de centre trivial. D'autre part, $\Sigma_{1}$ est l'ensemble des éléments de $\Xi$ qui sont nuls sur $\mathbb{R} \mathcal{P}$ et dont la restriction à $\boldsymbol{G}$ donne un poids de la représentation adjointé de $\boldsymbol{Q}$ dans $\boldsymbol{P}$. Il est alors clair que $\left\{\rho_{\lambda}, \lambda \in \Sigma_{1}\right\}$ n'est autre que l'ensemble des composés par $\left(I, \xi^{\prime}\right)$ d'une famille discriminante d'A-cocycles sur $B(G)$ associée à $R^{\prime} I^{G}$. Le corollaire (7.15) résulte alors de la proposition (7.13).

7.16. Remarques. - Soit $G$ un groupe de Lie ayant un nombre fini de composantes connexes dont la composante connéxe de l'unité dans $G$ est semi-simple (non nécessairement de centre fini). D'après la remarque (2.10) et le corollaire (7.15), il résulte que l'espace vectoriel des A-cocycles $K$-invariants sur la frontière maximale $B(G)$ de $G$ possède une base formée d'éléments $\mu$-négatifs stricts, pour tout mesure $\mu$ de $M^{1}(G)$ étalée et apériodique.

Dans [10] Furstenberg avait conjecturé l'existence, pour un groupe de Lie $G$ connexe semi-simple de centre fini, d'une base de l'espace vectoriel des A-cocycles $K$-invariants sur la frontière maximale $B(G)$ de $G$ formée d'éléments $\mu$-négatifs stricts pour toute mesure $\mu$ de $M^{1}(G)$ de "classe $B_{\infty}$ " (i.e. ayant une densité bornée et à support compact par rapport à la mesure de Haar sur G) . On a donc résolu et étendu cette conjecture.

D'autre part, la proposition (7.13) et le corollaire (7.14) étendent le corollaire du théorème 1 de [26] qui suppose que $\mu$ possède un moment d'ordre 2 et est absolument continue par rapport à la mesure de Haar.

7.17. Décomposition de G associée à une mesure $\mu$ étalée et apériodique de $M^{1}(G)$. - Soient $G$ un groupe de Lie ayant un nombre fini de composantes connexes; $\left\{\rho_{\lambda}, \lambda \in \Xi\right\}$ une famille discriminante $d$ ' $A$-cocycles associée à $G$; et $\mu$ une mesure de $M^{1}(G)$ étalée et apériodique. Posons

et

$$
\Xi_{-}^{\mu}=\left\{\lambda \in \Xi: \rho_{\lambda} \text { est } \mu \text {-négatif strict }\right\}
$$

$$
\Xi_{+}^{\mu}=\left\{\lambda \in \Xi: \rho_{\lambda} \text { est } \mu \text {-positif }\right\} \text {. }
$$

Des relations $\rho_{\lambda+\lambda},=\rho_{\lambda}+\rho_{\lambda},\left(\lambda, \lambda, \in \Xi, \lambda+\lambda^{\prime} \in \Xi\right), \rho_{\bar{\lambda}}=\overline{\rho_{\lambda}}(\lambda \in \Xi)$, il résulte, via la proposition (7.8), que $\left\{\Xi_{-}^{\mu}, \Xi_{+}^{\mu}\right\}$ est une partition de $\Xi$ stable par addition et conjuguaison. Nous notons alors

$$
M=M_{-}^{\mu} M_{+}^{\mu}, \quad D=D_{-}^{\mu} D_{+}^{\mu} \text { et } G=D_{-}^{\mu} E_{+}^{\mu} \text { avec } E_{+}^{\mu}=D_{+}^{\mu} K
$$

les décompositions de $M, D$ et $G$ associée à cette partition (voir \$ 4). 
D'autre part si $\alpha$ est un élément de $\Xi$ de la forme $\lambda-\gamma$ avec $\lambda \in \Xi$ et $\gamma \in \Sigma_{1}$ nous avons $\rho_{\alpha}=\rho_{\lambda} \rho_{\gamma}$; d'après ce qui prècède et le corollaire (7.15), on en déduit que $\left\{\Xi_{-}^{\mu}, \Xi_{+}^{\mu} \cup \Sigma_{2} \cup\{0\}\right\}$ est une partition de $\dot{\theta}=\Xi \cup \Sigma_{2} \cup\{0\}$ adaptée à la chambre de Weyl $W$ (déf. (5.1)). D'après la définition même des $\rho_{\lambda}, \lambda \in \Xi$, il est clair que la partition $\left\{\Xi_{-}^{\mu} \Xi_{+}^{\mu}\right\}$ est stable par automorphismes intérieurs (i.e. $\gamma \circ \operatorname{Adg} \in \Xi_{-}$(resp. $\Xi_{+}$) pour tous $\gamma \in \Xi_{-}$(resp. $\Xi_{+}$) et $g \in N(D)$, normalisateur de $D$ dans $G)$; comme tout automorphisme de $\boldsymbol{f}$ laissant stable $D$ permute les éléments de $\Sigma_{1}$ (démonstration analogue à celle du lemme $(2.8)$ j et par suite ceux de $\Sigma_{2}=-\Sigma_{1}$, on en déduit que cette partition de $\theta$ est stable par automorphismes intérieurs. Nous notons alors

$$
G=G_{-}^{\mu} G_{+}^{\mu} \text { avec } G_{-}^{\mu}=D_{-}^{\mu}=M_{-}^{\mu} N \text { et } G_{+}^{\mu}=D_{+}^{\mu} N \Gamma^{\prime}=\left(M_{+}^{\mu} P A\right) N \Gamma^{\prime} \text {, }
$$

la décomposition, modulo $m_{G}$, de $G$ associée à cette partition (voir $\$ 5$ ).

7.18. Remarque. - Pour une mesure $\mu$ de $M^{1}(G)$ donnée, les décompositions de $G$ associées à $\mu$ dépendent du choix de la décomposition

$$
y=\left(\mu_{+}+\rho\right) \oplus \mathcal{P} \boxplus Q \oplus K \text {. }
$$

Soit $y=\left(\mathscr{H}+\boldsymbol{Q}^{1}\right) \oplus \mathbb{d}^{1} \oplus \boldsymbol{U}^{1} \boxplus \boldsymbol{K}^{1}$ un autre choix de cette décomposition. On sait (remarque (1.11)) que ce nouveau choix se déduit du précédent par un automorphisme intérieur, Ad $x$, avec $x \in G_{0}$. La famille $\Xi$ est remplacée par $\Xi^{1}=\left\{\lambda \circ\right.$ Ad $\left.x^{-1}, \lambda \in \Xi\right\}$ et la famille d' A-cocycles $\left\{\rho_{\lambda} \lambda \in \Xi\right\}$ par la famille $\left\{\rho_{\lambda} \circ\left(J_{d}, I_{d}\right), \lambda \in \Xi\right\}$ où $x$ s'écrit $d k$ avec $d \in D$ et $k \in K$. (Voir lemme $(2.12))$. Nous avons

$$
\rho_{\lambda} \circ\left(J_{d}, I_{d}\right)(u, g)=\rho_{\lambda}(u, g)+\rho_{\lambda}(u \cdot g, d)-\rho_{\lambda}(u, d),
$$

pour tous éléments $u$ de $B(G)$ et $g$ de $G$.

Il s'ensuit que l'on a l'équivalence entre :

i) $\left\{\forall u \in B(G) ; \rho_{\lambda}\left(u, X_{n}\right\} \rightarrow(-\infty), \mathbb{P}_{g}-p . s . \forall g \in G\right\}$

ii) $\left\{\forall u \in B(G) ;\left[\rho_{\lambda} \circ\left(J_{d}, I_{d}\right)\right]\left(u, x_{n}\right) \rightarrow(-\infty), \mathbb{P}_{g}-p \cdot s, \quad \forall g \in G\right\}$.

D'après la remarque (4.5) les décompositions de $G$ obtenues avec ce nouveau choix se déduisent des précédentes par conjuguaison par $x \in G_{0}$. La décomposition de $G$ associée à $\mu$ est donc unique à conjuguaison par un élément de $G_{0}$ près. 


\section{EXISTENCE ET DESCRIPTION D'UNE $\mu$-FRONTIERE HOMOGENE MAXIMALE}

Nous désignons par G un groupe localement compact à base dénombrable.

8.1. - Soit $E$ un G-espace à gauche. On note ([7]) $C_{U}(E)$ l'ensemble des fonctions $f$ boréliennes bornées sur $E$ telles que l'application

$g \in G \longmapsto \sup _{x \in E}|f(g \cdot x)-f(x)|$ soit continue en $e$ (et donc U.C.G.). Muni de la norme de :a convergence uniforme, $C_{U}(E)$ est un espace de Banach. Si $E$ est un G-espace localement compact homogène, $C_{U}(E)$ est contenu dans $C(E)$, espace des fonctions continues sur E.

8.2. - Nous appelons $\mu$-espace de $G$ tout couple $(E, \nu)$ composé d'un G-espace à gauche localement compact $E$ et d'une mesure $\nu, \mu$-invariante (i.e. telle que $\mu * \nu=\nu)$, portée par $E$. Deux $\mu$-espaces de $G,(E, \nu)$ et $\left(E^{\prime}, \nu^{\prime}\right)$ sont dits $G$-isomorphes s'il existe un G-homéomorphisme $\phi$ de $E$ sur $E^{\prime}$ envoyant $\nu$ sur $\nu^{\prime}$. Un $\mu$-espace de $G,(E, v)$, est appelé une $\mu$-frontière de $G$ ([9]) si la suite de mesure de probabilité sur $E, X_{n} \nu$, converge, $\mathbb{P}_{g}-p . s$. $\forall g \in G$, vers une mesure ponctuelle, où $\left(\Omega, \mathcal{F},\left(x_{n}\right)_{n} \in \mathbb{N}^{\prime},\left(\mathbb{P}_{g}\right)_{g} \in G^{\prime}\right)$ désigne la marche aléatoire droite sur $G$ de loi $\mu$; si $E$ est homogène (resp. compact), nous disons que la $\mu$-frontière est homogène (resp. compacte).

8.3. - On sait ( $[8]$ ) que tout couple $(G, \mu)$ possède, à $G$-isomorphisme près, un unique $\mu$-espace compact, $\left(\pi_{\mu}, \nu\right)$ telle que 1 'application $f \longrightarrow f * v$ (voir (6.4)) soit une G-isométrie de $C\left(\Pi_{\mu}\right)$ sur $H_{\mu}$; en outre ([1]) si $\mu$ est étalée, cette application est une $G$-isométrie de $L^{\infty}\left(I_{\mu}, \varepsilon\right)$ sur $E_{\mu}$, où $\varepsilon$ est une mesure quasi-invariante sur $\Pi_{\mu} \cdot \Pi_{\mu}$ et $\nu$ sont appelés respectivement espace et noyau de Poisson de $\mu$ sur $G$. Si $\pi_{\mu}$ est métrisable, alors $\left(\pi_{\mu}\right.$, $\left.\nu\right)$ est une $\mu$-frontière compacte de $G$. Dans le cas où $G$ est soit un groupe de Lie connexe semi-simple de centre fini ([8]), soit un groupe de Lie connexe de type rigide ([1]), soit un groupe "localement produit direct de tels groupes" ( [4]), ( $\left.\pi_{\mu}, v\right)$ est une. $\mu$-frontière compacte et homogène pour toute mesure $\mu$ étalée sur $G$.

Dorériavant, G désigne un groupe de Lie ayant un nombre fini de composantes connexes. On désigne par $G_{0}$ la composante connexe de l'unité dans $G$ et par $R$ le radical de $G_{0}$. On note. $\xi$ l'application naturelle de $G$ sur $R l^{G}$ et $m_{G}$ une mesure de Haar sur G.

8.4. Théorème. - Soit G un groupe de Lie ayant un nombre fini de composantes 
connexes. Pour toute mesure $\mu$ de $M^{1}(G)$ étalée et ayant un moment d'ordre 1 , il existe un espace homogène $\Lambda_{\mu}(G)$ de $G$ (unique à un $G$-homéomorphisme près) et une mesure $\nu \in M^{1}\left(\Lambda_{\mu}\right), \mu$-invariante, tels que i) $\left(\Lambda_{\mu}, v\right)$ est une $\mu$-frontière homogène de G.

ii) L'application $f \longmapsto f * v$ est une G-isométrie de $L^{\infty}\left(\Lambda_{\mu}\right)$ sur l'espace de Banach, $E_{\mu}$, des fonctions $\mu$-harmoniques bornées, envoyant $C_{u}\left(\Lambda_{\mu}\right)$ sur le sousespace, $H_{\mu}$, de $\mathrm{E}_{\mu}$ formé des éléments uniformément continus à gauche.

\subsection{Description de $\Lambda_{\mu}$.}

1) Supposons que $\mu$ soit de plus apériodique et considérons la décomposition $G=G_{-}^{\mu} G_{+}^{\mu}$, modulo $m_{G}$, associée à $\mu$ (voir (7.17)). $\Lambda_{\mu}(G)$ est alors un revêtement à fibre dénombrable du G-espace homogène $G / G_{+}^{\mu}$. Plus précisément, soit $M=M_{-}^{\mu} M_{+}^{\mu}$ une décomposition de $M$ associée à $\mu$ (voir (.7.17)); en reprenant les notations antérieures, nous avons $G_{+}^{\mu}=M_{+}^{\mu}$ PAN ', alors

$$
\Lambda_{\mu}(G)=G / M_{+}^{\mu} \operatorname{PANT}_{\mu}^{\prime},
$$

où $\Gamma_{\mu}^{\prime}$ est un sous-groupe distingué de $\Gamma^{\prime}$ contenant $\Gamma_{0}^{\prime}$. Nous avons $\Lambda_{\xi(\mu)}\left({ }_{R}{ }^{G}\right)=\xi(G) /{ }^{G}\left(A N \Gamma_{\mu}^{\prime}\right)$; autrement dit $\Lambda_{\mu}(G)$ est homéomorphe en tant qu'espace topologique à $M_{-}^{\mu} \times \Lambda_{\xi(\mu)}\left({ }_{R}{ }^{G}\right)$.

2) Si $\mu$ n’est pas apériodique, soit $G_{\mu}$ le sous-groupe fermé de $G$ engendré par le support de $\mu$. Désignons par $s$ une section de l'application naturelle de $G$ sur $G / G_{\mu}$ (noter que $G / G_{\mu}$ est fini). Posons (voir [1] prop. IV.1) :

puis

$$
\sigma(g, x)=[s(g \cdot x)]^{-1} \quad g s(x) \quad\left(g \in G, x \in G / G_{\mu}\right) ;
$$

$$
g \cdot(x, y)=(g \cdot x, \sigma(g, x) \cdot y) \quad\left(g \in G,(x, y) \in G G_{G_{\mu}} \times \Lambda_{\mu}\left(G_{\mu}\right)\right) .
$$

Ainsi $G /_{G_{\mu}} \times \Lambda_{\mu}\left(G_{\mu}\right)$ est muni d'une structure de G-espace. Nous avons alors

$$
\Lambda_{\mu}(G)=G /_{G} \times \Lambda_{\mu}\left(G_{\mu}\right)
$$

Les paragraphes 9 et 10 sont consacrés à la démonstration du théorème (8.4). Ce théorème sera ensuite étendu au cas des groupes L.C.0. (voir § 13).

8.6. Définition. - Nous disons qu'une $\mu$-frontière homogène, $(\Lambda, v)$, de $G$ est maximale, si pour toute autre $\mu$-frontière homogène $(E, \lambda)$ de $G$, il existe une application surjective continue de $\Lambda$ sur $E$, commutant avec les opération de $G$ et envoyant $\nu$ sur $\lambda$. 
8.7. Proposition. - Soient $(G, \mu)$ vérifiant les hypothèses du théorème (8.4) et $\left(\Lambda_{\mu}, \nu\right)$ la $\mu$-frontière homogène de $G$ associée à $(G, \mu)$ par ce théorème. Alors $\left(\Lambda_{\mu}, \nu\right)$ est une. u-frontière homogène maximale de $G$. 


\section{CONVERGENCE DE CERTAINES COMPOSANTES DE $x_{n}$}

Dans ce qui suit $G$ désigne un groupe de Lie ayant un nombre fini de composantes connexes ; et ' $\mu$ une mesure de $M^{1}(G)$ étalée, apériodique, possèdant un moment d'ordre 1 .

9.0. Bref rappel des notations antérieures : On désigne par $y=R \boxplus \rho$ une décomposition de Lévi de l'algèbre de Lie $y$ de $G$; par $\mathcal{S} \mathcal{P}_{\mathbb{P} \boxplus} \boldsymbol{K}$ une décomposition d'Iwasawa de la sous-algèbre de Lévi $\mathcal{f}$ de $\mathcal{f}$; on obtient la décomposition $\mathscr{J}=\mathbb{D} \mathbb{H}$, où $D=R \boxplus \mathcal{N} \boxplus Q$ est une sous-algèbre résoluble et $K$ une sous-algèbre compacte de $\mathcal{H}$.

Soit $\mathcal{M}$ le nilradical de $\boldsymbol{R}$ et $\mathcal{S}$ une sous-algèbre de Cartan du centralisateur $\zeta$ de $S$ dans $R$. Nous avons $R=\mu+Q$ et $D=h+(Q \otimes Q)$, où $\xi=\mu \boxplus \mathcal{N}$ est le nilradical de $D$.

Soit $\Xi$ (resp. $\Lambda$ ) l'ensemble des poids de la représentation adjointe de $D$ dans $h^{\mathbb{C}}$ (resp. $\boldsymbol{H}^{\mathbb{C}}$ ). A la mesure $\mu$ correspond la partition $\left\{\Xi_{-}^{\mu}, \Xi_{+}^{\mu}\right\}$ de $\Xi$ définie par :

$$
\begin{array}{ll}
\Xi_{-}^{\mu}=\{\lambda \in \Xi: & \left.a_{\lambda}=\iiint_{\lambda}(u, g) \quad v(d u) \quad \mu(d g)<0\right\} \\
\Xi_{+}^{\mu}=\{\lambda \in \Xi: & \left.a_{\lambda}=\iint \rho_{\lambda}(u, g) \quad v(d u) \quad \mu(d g) \geq 0\right\},
\end{array}
$$

où $\nu$ désigne l'unique mesure $\mu$-invariante sur $\mathrm{E}(\mathrm{G})$ (voir (7.17.)). Cette partition est stable par addition et conjuguaison. De plus si $\Sigma_{1}$ (resp. $\theta$ ) désigne l'ensemble des poids de la représentation adjointe de $D$ dans $(\xi / \mu)^{\mathbb{C}}$ (resp. dans $\mathcal{H}^{\mathbb{C}}$ ), $\left\{\Xi_{-}, \Xi_{+} \cup\left(-\Sigma_{1}\right\} \cup\{0\}\right\}$ est une partition de $\theta$ adaptée à la chambre de Weyl $W$ (qui a servi à définir la décomposition d'Iwasawa $\mathcal{S}=\mathcal{N} \oplus Q \mathbb{K}$ de $\mathcal{S}(\operatorname{voir}(1.2$.$) ) et stable par automorphismes inté-$ rieurs. (voir (7.17.)) .

$\left\{\Lambda \cap \Xi_{-}, \Lambda \cap \Xi_{+}\right\}$est alors une partition stable par addition et conjuguaison de $\Lambda$, soit $\mu^{+}=\mu_{-}^{\mu} \oplus \mu_{+}^{\mu}$ la décomposition de $\mu$ associée à cette partition (voir (3.7.)). Les décompositions de $\boldsymbol{h}$ et $D$ associées à la partition $\left\{\Xi_{-}^{\mu}, \Xi_{+}^{\mu}\right\}$ sont alors (voir $\$ 4$ et 5 )

$$
\begin{aligned}
& \xi=h_{-}^{\mu} \oplus h_{+}^{\mu} \text { avec } h_{-}^{\mu}=\mu_{-}^{\mu} \rightarrow \mathcal{N}_{-} \text {et } h_{+}^{\mu}=\mu_{+}^{\mu} \\
& D=D_{-}^{\mu} \oplus D_{+}^{\mu} \text { avec } D_{-}^{\mu}=h_{-}^{\mu} \text { et } D_{+}^{\mu}=\left(h_{+}^{\mu}+\varphi^{\mu}\right) \circledast Q
\end{aligned}
$$

Nous savons que, muni du produit $x$ défini par la formule (3.3.), $h$ (resp. $\mathcal{M}$ ) est un groupe nilpotent qui est le produit amalgamé des sous-algèbres $h_{-}^{\mu}$ et $h_{+}^{\mu}$ (resp. $\mu_{-}^{\mu}$ et $\mu_{+}^{\mu}$ ) ; en outre les projections $\rho_{-}$et $\rho_{+}$(resp. $\rho_{-}^{\prime}$ et $\rho_{+}^{\prime}$ ) associées à ce produit amalgamé sont polynomiales (voir (3.7.)). 
Désignons par $M, P, N, A, K, D$, $D_{-}^{\mu}$ et $D_{+}^{\mu}$ les sous-groupes de Lie connexes de $G$ ayant respectivement pour algèbre de Lie $\mu, \mathcal{S}, \mathcal{N}, \mathcal{Q}, \mathcal{K}$, $\varpi$, $D_{-}^{\mu}$ et $D_{+}^{\mu}$. Notons $\Gamma$ le centralisateur de $A$ dans $K$ et $\Gamma^{\prime}$ l'intersection des normalisateurs dans $G$ de $N, A, K$ et $P$.

$\mathrm{K} \Gamma^{\prime}$ est alors un sous-groupe fermé de $G$ ayant un nombre fini de composantes connexes ; on choisit un de ses sous-groupes compacts maximaux qui sont conjugués deux à deux, soit $L$. En posant $K=K L$, on obtient la décomposition $G=\square K$, où D est un sous-groupe fermé résoluble connexe, l'image de $K$ par la représentation adjointe de $G$ est compacte et $D \cap K$ est contenu dans $P$.

D'autre part, posons $M_{-}^{\mu}=\exp \mu_{-}^{\mu}$ et $M_{+}^{\mu}=\exp \mu_{+}^{\mu}$. $M_{-}^{\mu}$ et $D_{-}^{\mu}=M_{-}^{\mu}$ sont. des sous-groupes fermés simplement connexes de $G$. M (resp. D) est le produit amalgamé des sous-groupes $M_{-}^{\mu}$ et $M_{+}^{\mu}$ (resp. $D_{-}^{\mu}$ et $D_{+}^{\mu}=M_{+}^{\mu} P A$ ).

Enfin, en posant $G_{-}^{\mu}=D_{-}^{\mu}$ et $E_{+}^{\mu}=D_{+}^{\mu} K=D_{+}^{\mu} K \Gamma^{\prime}$, l'application $\eta: G_{-}^{\mu} \times E_{+}^{\mu} \longrightarrow G$ est un isomorphisme de variétés analytiques; nous notons

$(d, g) \longmapsto d g$

$\boldsymbol{\mu}_{\text {- }}$ et $\boldsymbol{\mu}_{+}$les projections de $G$ sur $G_{-}^{\mu}$ et $E_{+}^{\mu}$ telles que $g=\mu_{-}(g) \mu_{+}(g)$ pour tout $g$ de $G$.

Nous allons à présent construire une décomposition de $G$ un peu plus fine que celle $G=D_{-} D_{+} K$ obtenue ci-dessus. Nous omettons l'indice " $\mu$ ".

9.1.- $\zeta_{+}$est un idéal nilpotent de $D_{+}$et $\Xi_{+}$est l'ensemble des poids de la représentation adjointe de $D_{+}$dans $h_{+}$. Posons $\Xi_{0}=\left\{\lambda \in \Xi ; a_{\lambda}=0\right\}$ et $\Xi_{+}^{*}=\left\{\lambda \in \Xi ; a_{\lambda}>0\right\},\left\{\Xi_{+}^{*}, \Xi_{0}\right\}$ est alors une partition de $\Xi_{+}$stable par addition et conjuguaison; notons respectivement

$$
\eta_{+}=\eta_{0} \oplus \eta_{+}^{*}, D_{+}=D_{0} \oplus D_{+}^{*} \text { et } D_{+}=D_{0} D_{+}^{*}
$$

les décompositions de $\boldsymbol{h}_{+}, \mathbb{D}_{+}$et $D_{+}$associées à cette partition de $\Xi_{+}$. Comme on a

$$
\left.\begin{array}{l}
\lambda \in \Xi_{0} \\
\lambda^{\prime} \in \Xi_{-} \quad\left(\operatorname{resp} . \Xi_{+}^{*}\right) \\
\lambda+\lambda^{\prime} \in \Xi
\end{array}\right\} \Longrightarrow \lambda+\lambda^{\prime} \in \Xi_{-}\left(\operatorname{resp} \cdot \Xi_{+}^{*}\right),
$$

il s'ensuit que $\eta_{*}$ (resp. $D_{0}, D_{0}$ ) normalise $\eta_{-}$et $\boldsymbol{\eta}^{*}$ (resp. $\boldsymbol{D}_{-}$et $D_{+}^{*}, D_{-}$et $\left.D_{+}^{*}\right)$.

$\boldsymbol{h}$ est alors somme directe des trois sous-algèbres $\boldsymbol{h}_{-}$, ho et $\boldsymbol{h}_{+}^{*}$.

Muni du produit $x$ défini par la formule (3.3.), $\boldsymbol{l}$ est le produit amalgamé des sous-groupes $\boldsymbol{h}_{-}, \boldsymbol{h}_{\mathrm{o}}$ et $\boldsymbol{h}_{+}^{*}$; en outre les projections $\rho_{-}$, $\rho_{0}$ et $\rho_{+}$ associées à ce produit amalgamé sont polynomiales .

$D$ est le produit amalgamé des sous-groupes $D_{-}, D_{0}$ et $D_{+}^{*}$; $D_{-}$et $D_{+}^{*}$ sont simplement connexes et $D_{0}$ normalise $D_{-}$et $D_{+}^{*}$ con notera que $D_{-}$et $D_{+}^{*}$ jouent des rôles symétriques). Nous avons $D_{-}=\exp \xi_{-}, D_{0}=\left(\exp \bar{\zeta}_{0}\right) P A$ et 
$\square_{+}^{*}=\exp \xi_{+}^{*}$.

Tout élément $g$ de $G$ s'écrit alors sous la forme $g=d_{-} d_{0} d_{+} k$ avec $d_{-} \in D_{-}$, $d_{0} \in D_{0}, d_{+} \in D_{+}^{*}$ et $k \in K$. Comme $D \cap K$ est contenu dans $P$, les composantes $d_{-}$et $d_{+}$sont uniquement définies par $g$. Si d est un élément $D$, nous noterons respectivement $\boldsymbol{\mu}_{-}(d), \boldsymbol{p}_{q^{*}}(d)$ eet $\boldsymbol{\mu}_{+}^{*}(d)$ les éléments de $D_{-}$, $D_{0}$ et $D_{+}^{*}$ tels que $d=\mu_{-}(d) \mu_{0}(d) \mu_{+}^{*}(d)$.

9.2. Théorème.- Soient G un groupe de Lie ayant un nombre fini de composantes connexes et $\mu$ une mesure de $M^{1}(G)$ étalée apériodique et possédant un moment d'ordre 1 . Alors avec les notations de (g.1.) nous avons

i) Pour tout $g$ de $G$, la composante $\mu_{-}\left(x_{n} g\right)$ de $x_{n} g$ sur $D_{-}\left(=G_{-}\right)$converge, $\mathbb{P}_{\mathrm{X}}$ - p.s. $\forall \mathrm{x} \in \mathrm{G}$, vers une v.a. indépendante de $\mathrm{g}$.

ii) Pour tout $g$ de $G$, la composante $\mu_{+}\left(g x_{n}\right)$ de $g x_{n}$ sur $D_{+}^{*}$ converge, $\mathbb{P}_{x}$ - p.s. $\forall x \in G$, vers une v.a. indépendante de $G$.

La démonstration de ii) étant analogue à celle de ij., nous ne donnerons que la preuve détaillée de ij. Nous dirons ensuite quelques mots sur la preuve de ii).

Nous commençons par introduire les éléments nécessaires à cette démonstration. On peut oublier (9.1.) et n'avoir en tête que les notations de (9.0.).

9.3.- Fonctions polynomiales sur $h$ invariantes à droite par $\xi_{+}$: Le groupe $G_{-}=D_{-}=\exp h_{\text {_ }}$ est nilpotent simplement connexe; $G_{-}$est donc isomorphe au groupe $\left(\xi_{-}, x\right) \cdot \xi_{\text {- }}$ apparaît comme un D-espace homogène à gauche D-homéomorphe à $\mathrm{C}_{\mathrm{D}_{+}}$; l'action de $\mathrm{D}$ sur $\boldsymbol{h}_{\text {- étant définie par }}$

$$
d \cdot u=\exp _{G_{-}}^{-1} \circ p_{-}(d \exp u) \quad\left(d \in D, u \in \zeta_{-}\right) .
$$

Etendons cette définition à $G$ en posant

$$
\mathrm{g} \cdot u=\exp _{G_{-}}^{-1} \circ \mu_{-}(\mathrm{g} \exp u) \quad\left(\mathrm{g} \in \mathrm{G}, u \in \zeta_{-}\right) .
$$

Comme en général. $E_{+}$n'est pas un sous-groupe de $G, G$ n'opère pas ainsi sur

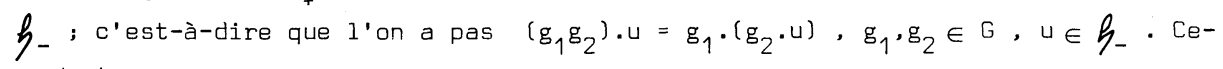
pendant nous avons

$$
\text { (*) } \quad(d g) \cdot u=d .(g . u) \quad\left(d \in D, g \in G, u \in \zeta_{-}\right) .
$$

Il suffit en effet de remarquer que puisque $D_{+} E_{+} \subset E_{+}$, on a

$$
\mu_{-}(d g)=\mu_{-}\left(d \mu_{-}(g)\right) \quad(d \in D, g \in G) .
$$

Consitérons l'ensemble $\mathrm{J}$ des fonctions polynomiales, à valeurs complexes, sur $h$ qui sont invariantes à droite par $h_{+}$(i.e. $T \in J$ si $T \circ R_{c}=T$, $\forall c \in h_{+}(\operatorname{voir}(3))$. Soit $T \in J$ et $u \in \zeta^{+}$; écrivons $u=r_{-}(u) x_{+}(u)$. Nous avons

$$
T(u)=T\left(\rho_{-}(u) \times \rho_{+}(u)\right)=T\left(\rho_{-}(u)\right) .
$$


car $T$ est invariant par $h_{+}$; il s'ensuit que l'on a $T=T \boldsymbol{b}_{-} \circ \rho_{-}$, où où $T \mathfrak{h}$ - désigne la restriction à $\boldsymbol{h}$ - de $T$. Par suite $\mathcal{J}$ est l'ensemble des fonctions polynomiales sur $h$ de la forme T' o $\rho_{-}$où T' est une fonction polynomiale sur $\boldsymbol{h}_{-}$.

Pour $T \in J$. et $g \in G$ définissons

$$
T \cdot g: u \in h \longrightarrow T\left(g \cdot \rho_{-}(u)\right) \text {. }
$$

De la relation (*) il résulte que si $g=d k$ avec $d \in D$ et $k \in K$ nous avons $T \cdot g=(T \cdot d) \cdot k$.

D'autre part comme $D=(\exp \boldsymbol{h}) P A$, écrivons $d=(\exp v) x$ avec $v \in \boldsymbol{h}$ et $x \in P A$; nous avons pour tout $u$ de $h$

$$
d \exp u=\exp v\left(x(\exp u) x^{-1}\right) \chi
$$

$$
=\exp v(\exp \operatorname{Ad} \chi(u)) \chi
$$

$\zeta$ étant un idéal de $D$, Ad $x \cdot(u) \in \xi$; d'après la formule de Campel1-Hausdorff nous avons

$$
\begin{aligned}
d \exp u & =\exp (v \times \operatorname{Ad} x \cdot(u)) \chi \\
& =\exp \left(\rho_{-}(v \times \operatorname{Ad} x(u)) \times \rho_{+}(v \times \operatorname{Ad} x(u))\right) x \\
& =\left(\exp \rho_{-}(v \times \operatorname{Ad} x \cdot(u))\right)\left(\exp \rho_{+}(v \times \operatorname{Ad} x(u)) x .\right.
\end{aligned}
$$

Par suite

et

$$
\mu_{-}(d \exp u)=\exp \left(\rho_{-}(v \times \operatorname{Ad} x(u))\right)
$$

Or

$$
d \cdot \rho_{-}(u)=\exp _{G_{-}^{-1}}^{-1} \circ p_{-}\left(d \exp \left(\rho_{-}(u)\right)\right)=\rho_{-}\left(v \times \operatorname{Ad} x\left(\rho_{-}(u)\right)\right) .
$$

$$
\begin{aligned}
\operatorname{Ad} x(u) & =\operatorname{Ad} x\left(\rho_{-}(u) \times \rho_{+}(u)\right) \\
& =\operatorname{Ad} x\left(\rho_{-}(u)\right) \times \operatorname{Ad} x\left(\rho_{+}(u)\right)
\end{aligned}
$$

car Ad $x$ est un automorphisme d'algèbre de Lie de $h$.

comme $h_{\text {- et }} h_{\text {+ sont stables par }} \rho \oplus Q$, il s'ensuit que

$$
\text { Ad } x\left(\rho_{-}(u)\right) \in h_{-} \text {et } \operatorname{Ad} x\left(\rho_{+}(u)\right) \in h_{+} \text {. }
$$

Autrement dit nous avons

$$
\begin{aligned}
& \rho_{-}(A d x(u))=A d x \text { o } \rho_{-}(u) \\
& \rho_{+}(A d x(u))=A d x \quad 0 \quad \rho_{+}(u) \text {. }
\end{aligned}
$$

Finalement nous obtenons

$$
\begin{aligned}
d \cdot \rho_{-}(u) & =\rho_{-}\left(v \times \operatorname{Ad} \times\left(\rho_{-}(u)\right)\right) \\
& =\rho_{-}\left(v \times \rho_{-}(\operatorname{Ad} x(u))\right) \\
& =\rho_{-}(v \times \operatorname{Ad} \times(u)) .
\end{aligned}
$$

D'où l'on déduit

(**) $T . d=T \circ L_{V} \circ A d x \circ \rho_{-}=T \circ L_{V} \circ \operatorname{Ad} x(T \in J)$ (voir \$3).

On voit donc que $T . d$ est un élément de $J$; $D$ opère donc à droite sur $J$;

$J$ est un D-espace à droite.

On notera qu'en général $T . k$ n'est pas un polynôme pour $k \in K$. C'est seulement 
une fonction analytique $f$ sur $\zeta$ définie par

$$
f: u \longmapsto T\left(\exp _{G_{-}}^{-1} \circ p_{-}(k \exp u)\right)
$$

et vérifiant $f\left(u_{-} \times u_{+}\right)=f\left(u_{-}\right)^{-}\left(u_{-} \in h_{-}, u_{+} \in h_{+}\right)$.

La raison en est que $h$ est un idéal de $\mathcal{D}$ mais n'est pas en général un idéal de $\mathcal{f}$. Dans le cas où $\mathcal{h}$ est un idéal de $\mathcal{f}$, c'est-à-dire quand $\mathcal{f}$ est moyennable, on voit facilement que le groupe $G$ opère sur $J$; $J$ est alors un G-espace à droite.

Pour tout entier naturel $\mathrm{r}$, appelons $\mathrm{J}_{\mathrm{r}}$ le sous-espace vectoriel de $\mathrm{J}$ formé des éléments de degré inférieur ou égal à $r$. D'après la relation (**) et le lemme (3.5.), les sous-espaces vectoriels $J_{r}, r \in \mathbb{N}$, de $J$ sont invariants par les opérations de $D$.

Choisissons une base adaptée (voir $\{3)\left(e_{i}\right)_{1}<i<m$ de $h_{-}^{\mathbb{C}}$ qui triangularise simultanément les éléments de $\left.\operatorname{ad}(Q \otimes Q)\right|_{\xi_{-}^{\mathbb{C}}}$ et AdPA| $\boldsymbol{h}_{-}^{\mathbb{C}}$. Pour $\lambda \in \Xi$, désignons par $\phi_{\lambda}$ le poids de la représentation adjointe de $\mathrm{D}$ dans $\boldsymbol{h}^{\mathbb{C}}$, dont $\lambda$ est la différentielle en e (voir (2.9.)). Si $\chi \in$ PA dans la base choisie de $h_{-}^{\mathbb{C}}$. Ad $x$ est une matrice triangulaire inférieure dont les éléments diagonaux sont $\left\{\phi_{\lambda}(x), \lambda \in \Xi_{-}\right\}$.

Considérons l'ensemble $\mathbb{N}^{m}$. Si $\ell=\left(\ell_{1}, \ldots, \ell_{n}\right) \in \mathbb{N}^{m}$ nous posons

$$
\|\ell\|=\left(\ell_{1}+\ldots+\ell_{i_{1}}+2\left(l_{i_{1}+1}+\ldots+\ell_{i_{2}}\right)+\ldots+p\left(l_{i_{p-1}}+1+\ldots+\ell_{m}\right)\right.
$$

(voir $\$ 3$ ) et nous ordonnons $N^{m}$ par la relation d'ordre total définie par

$$
\ell<\ell^{\prime} \Longleftrightarrow\left\{\begin{array}{l}
\|\ell\|<\left\|\ell^{\prime}\right\| \\
\|\ell\|\|=\| \ell^{\prime} \| \text { avec le premier } i \text { tel que } \ell_{i} \neq \ell^{\prime} \\
\text { vérifie } \ell_{i}<\ell^{\prime}{ }_{1}
\end{array}\right.
$$

Soit $\left(x_{i}\right)_{1} \leq i \leq m$ le système de fonctions coordonnées associé à la base $\left(e_{i}\right)_{1} \leq i \leq m$. Pour $\ell=\left(\ell_{1}, \ldots, \ell_{m}\right) \in \mathbb{N}^{m}$, nous notons $\chi^{\ell}$ la fonction monome $x_{1}^{l_{1}} \ldots x_{m}^{\ell}$; nous avons $d g \chi^{\ell}=\|l\|$. Pour $r \in \mathbb{N}^{\star}$; nous choisissons pour base de $J_{r}$ la base ordonnée $\left\{1, \chi^{\ell} \circ \rho_{-}, \ell \in \mathbb{N}^{m}-\{0\}\right.$, $\left.\| \ell \leq r\right\}$. Nous notons $J_{r}^{*}$ le sous-espace de $J_{r}$ engendré par $\left\{\chi^{\ell}\right.$ op,$\ell \in \mathbb{N}^{m}-\{0\}$, $\left.\left\|_{\ell}\right\| \leq r\right\}$.

$$
\begin{aligned}
& \text { Soit } T_{1} \in J \text {; nous avons pour } d=(\exp v) x \in D \text { avec } v \in h \text { et } x \in P A \text {, } \\
& T_{1} \cdot d=T_{1} \circ L_{V} \circ \text { Ad } x \text {. } \\
& =\left(T_{1}+T_{2}\right) \circ \text { Adx } x \text {, où } T_{2} \in J \text { avec dg } T_{2}<d g T_{1} \text { (lemme }
\end{aligned}
$$

(3.5.)) . Par suite

$$
T_{1} \cdot d=T_{1} \circ A d x+T_{2} \circ A d x
$$

avec

$$
\begin{aligned}
\operatorname{dg} T_{2} \circ \text { Ad } x & \left.=d g T_{2} \quad \text { (lemme }(3.5 .)\right) \\
& <d g T_{1}
\end{aligned}
$$




$$
\begin{aligned}
& \text { Ecrivons } T_{1}=T_{1}^{\prime} \circ \rho_{-} \text {où } T^{\prime}{ }_{1} \text { est la restriction de } T_{1} \text { à } h \text { - ncus avons } \\
& T_{1} \circ \operatorname{Ad} x=T^{\prime}{ }_{1} \circ \rho_{-} \circ \text { Ad } x \\
& \left.=T^{\prime}{ }_{1} \circ \text { Ad } x \text { o } \rho_{-} \text {(car nous avons vu que } \rho_{-} \circ A d \chi=A d \chi_{O} \rho_{-}\right) \text {. }
\end{aligned}
$$

Il est alors clair que dans la base ordonnée choisie de $\mathcal{J}_{r}$, l'action d'un élément $d$ de $D$ est représenté par une matrice $M(d)$ triangulaire supérieure ayant 1 puis des produits de la forme $\phi_{\lambda_{1}}(d) \ldots \phi_{\lambda}(d)$, où $\lambda_{1}, \ldots, \lambda_{s} \in \Xi_{-}$(les poids $\lambda_{i}$ ne sont pas nécessairement distincts\}, pour éléménts diagonaux. Plus précisément, notons $\phi_{\lambda_{i}}$ le poids qui est associé al vecteur propre $e_{i}$ de la représentation adjointe de $P A$ cans $\boldsymbol{H}_{-}^{\mathbb{C}}$; les $\phi_{\lambda_{i}}$ ne sont pas tous distincts et la famille $\left\{\phi_{\lambda}, 1 \leq i \leq m\right\}$ n'est autre que $l$ 'ensemble des restrictions à $P A$ des poids $\left\{\phi_{\lambda}, \lambda \in \hat{E}_{-}\right\}$. Alors pour $\ell=\left(l_{1}, \ldots, \ell_{m}\right\} \in \mathbb{N}^{m}$ la fonction polynomiale $\chi^{\ell} \circ \rho_{-} d_{\ell} J$ est pour l'action de $D$, un vecteur presque propre associé au poids $\phi_{\lambda_{1}}^{\ell} \ldots \phi_{\lambda_{m}}^{\ell_{m}}$

Nous allons à présent établir un lemme qui nous servira pour montrer le théorème..

9.4. Lemme. - Soient $\left(A_{i}\right)_{i}>0$ une suite de matrices carrées triangulaires supémeures d'ordre $q$ et $\Pi_{k}=A_{k} \ldots A_{0}$. Si les éléments diagonaux $\left(\delta_{i}^{j}\right)_{1}^{j} \leq j \leq q$
des $A_{i}$ sont tels que $\lim _{k} \sqrt[k]{\left|\delta_{k}^{j} \ldots \delta_{j}^{j}\right|}=a_{j}$ avec $0<a_{j}$, pour tout $j \in\{1, \ldots, q\}$, et $s i \overline{\lim }_{k}\left\|A_{k}\right\| \frac{1}{k} \leq 1$, alors $\lim _{k}\left\|\Pi_{k}\right\| \frac{1}{k}$ existe et vaut $a=\sup _{1 \leq j \leq q} a_{j}$.

Preuve. - Nous allons raisonner par récurrence sur la dimension q des matrices $A_{i}$.

Si $q=1$, le lemme est trivial; supposons qu'il soit vrai pour des matrices d'ordre strictement inférieur à $\ell$ et considérons des matrices $\left(A_{i}\right)_{i \geq 0} d$ ordre $\ell$. Posons

$$
A_{i}=\left[\begin{array}{l|l}
d_{i} & w_{i} \\
\hline 0 & B_{i}
\end{array}\right] \quad(i \geq 0)
$$

Nous avons

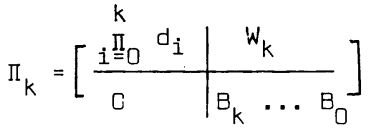

avec $w_{k}=\sum_{s=0}^{k} d_{k} \cdots d_{s+1} w_{s} B_{s-1} \ldots B_{1} ; d^{\prime}$ où

$$
\left\|w_{k}\right\| \leq \sum_{s=0}^{k}\left|d_{k}\right| \ldots|| d_{s+1} \mid\left\|w_{s}\right\|\left\|B_{s-1} \ldots B_{0}\right\| .
$$


Q'après les hypothèses faites nous avons

$$
\begin{gathered}
V_{\varepsilon}>0 \exists s_{0} \in \mathbb{N}^{*}, \forall s \geq s_{0} b-\varepsilon<\left\|_{s} \ldots B_{0}\right\| 1 / s<b+\varepsilon \\
a_{1}-\varepsilon<\left|d_{s} \ldots d_{0}\right|^{1 / s}<a_{1}+\varepsilon \\
\|_{w_{s} \|}{ }^{1 / s}<1+\varepsilon
\end{gathered}
$$

où $b=\sup _{2 \leq j \leq q} a_{j}$.

$$
\begin{aligned}
& \text { D'où pour } k>s_{0} \text {, } \\
& \left\|w_{k}\right\| \leq\left|d_{k}\right| \ldots\left|d_{0}\right|\left(\sum_{s=0}^{k}\left(\left|d_{0}\right| \ldots\left|d_{s}\right|^{-1}\left\|w_{s}\right\|\left\|B_{s-1} \ldots B_{0}\right\|\right)\right) \\
& \leq\left|d_{k}\right| \ldots\left|d_{0}\right|\left(\sum_{s=0}^{s}()+\sum_{s_{0}+1}^{k}()\right) \\
& \leq\left(a_{1}+\varepsilon\right)^{k}\left(C^{\prime}(\varepsilon)+\sum_{s=1}^{k}\left(a_{1}-\varepsilon\right)^{-s}(b+\varepsilon)^{s-1}(1+\varepsilon)^{s}\right)
\end{aligned}
$$$$
\text { où } C^{\prime}(\varepsilon) \text { est une constante ne dépendant que de } \varepsilon \text {. }
$$$$
\leq\left(a_{1}+\varepsilon\right)^{k}\left[C^{\prime}(\varepsilon)+\frac{1+\varepsilon}{\left(a_{1}-\varepsilon\right)-(b+\varepsilon)(1+\varepsilon)}\left(1-\left(\frac{(b+\varepsilon)(1+\varepsilon)}{a_{1}-k}\right)\right]\right.
$$

et pour tout $\varepsilon>0$ assez petit, il vient

$$
\overline{\lim }_{k}\left\|W_{k}\right\| 1 / k \leq\left\{\begin{array}{l}
\left(a_{1}+\varepsilon\right) \frac{(b+\varepsilon)(1+\varepsilon)}{a_{1}-\varepsilon} \text { si } \frac{(b+\varepsilon)(1+\varepsilon)}{a_{1}-\varepsilon}>1 \\
a_{1}+\varepsilon \text { si } \frac{(b+\varepsilon)(1+\varepsilon)}{a_{1}-\varepsilon}<1
\end{array}\right.
$$

Par suite $\overline{\lim }\left\|w_{k}\right\|^{1 / k} \leq \sup \left\{a_{1}, b\right\}=a$ et donc $\lim _{k}\left\|\Pi_{k}\right\| 1 / k=a$. Le lemme est démontrék.

9.5. Démonstration du i) du théorème (9.2.). Appelons p l'application naturelle de $G$ sur $D^{G}$ et considérons une section mesurable $s$ de $P$ à valeurs dans $K$. Tout $g \in G$ s'écrit alors

$$
g=d(g)(\operatorname{sop}(g))
$$

avec $d(g)=g(\operatorname{sop}(g))^{-1} \in D$ et $\operatorname{sop}(g) \in K$.

Ecrivons $X_{n}=X_{0} Y_{1} \ldots Y_{n}$ où les $Y_{i}$ sont des variables aléatoires indépendantes et de loi $\mu$. Posons

$$
\begin{aligned}
& d_{i}=d\left(Y_{i}\right), k_{i}=\operatorname{sop}\left(Y_{i}\right) \quad i \in\{1, \ldots, n, \ldots\} \\
& D_{i}=d\left(X_{i}\right), k_{i}=\operatorname{sop}\left(X_{i}\right) i \in\{0, \ldots, n, \ldots\} .
\end{aligned}
$$


Nous avons

$$
\begin{aligned}
& Y_{i}=d_{i} k_{i} \text { avec } d_{i} \in D \text { et } k_{i} \in K \quad i \in\{1, \ldots, n, \ldots .\} \\
& x_{i}=D_{i} K_{i} \text { avec } D_{i} \in D \text { et } k_{i} \in K \quad i \in\{0, \ldots, n, \ldots\} \text {. }
\end{aligned}
$$

Soit $r \in \mathbb{N}^{*}$ et $T \in Y_{r}$. Nous avons

$$
T \cdot x_{n}(u)=\left(T \cdot D_{n}\right)\left(K_{n} \cdot \rho_{-}(u)\right)=M\left(D_{n}\right)(T)\left(K_{n} \cdot \rho_{-}(u)\right)(u \in \xi) \text {. }
$$

$M\left(D_{n}\right)$ est une matrice triangulaire supérieure dont les éléments diagonaux sont 1 et des produits de la forme $\phi_{\lambda_{1}}\left(D_{n}\right) \ldots \phi_{\lambda_{t}}\left(D_{n}\right)$, où $\lambda_{1}, \ldots, \lambda_{t} \in \Xi_{-}$.

Soit $\lambda \in \Xi$, nous avons $(\operatorname{voir}(2.4$.$) et (2.9)$.$) .$

$$
\log \left|\phi_{\lambda}\left(D_{n}\right)\right|=\tau_{\lambda}\left(v_{0}, x_{n}\right),
$$

où $U_{0}$ est l'élément de $B(G)$ stable par $N(D)$ (lemme (2.3.)) et $v_{0}=\left(u_{0}, \zeta(e)\right)$.

D'après le lemme (7.7.), il s'ensuit que

$\frac{1}{n} \log \left|\phi_{\lambda}\left(D_{n}\right)\right|=\frac{1}{n} \tau_{\lambda}\left(v_{0}, x_{n}\right) \stackrel{\mathbb{P} g-p \cdot s \cdot \forall g \in G}{\longrightarrow} a_{\lambda}=\delta_{G} \delta_{G_{0} G^{G}} \delta_{B} \tau_{\lambda}((u, x), g) d x$ où $\nu$ désigne l'unique mesure de probabilité $\mu$-invariante sur $B(G)$ et $d x$ la mesure de Haar normalisée sur $G_{0}{ }^{G}$.

Nous avons :

$$
a_{\lambda}=\int_{B} \int_{G} \rho_{\lambda}(u, g) \nu(d u) \mu(d g) \quad(\operatorname{voir}(2.9 .)) \text {. }
$$

D'après la définition de la partition $\left\{\Xi_{-}, \Xi_{+}\right\}$de $\Xi$ associée à la mesure $\mu$ de $M^{1}(G)$ (voir $(7.17$.$) ), dire que \lambda \in \Xi_{-}$c'est-à-dire que l'A-cocycle $\rho_{\lambda}$ est $\mu$-négatif strict ou encore (proposition (7.12.)) que $a_{\lambda}<0$.

Autrement dit, les éléments diagonaux de $M\left(D_{n}\right)$ sont 1 et des éléments $\delta_{n}^{i}$ tels que $\lim \left|\delta_{n}^{i}\right|^{1 / n}$ existe et est strictement inférieur à $1, \mathbb{P}_{g}-p \cdot s . \forall g \in G$. D'autre part, pour. $g \in G$, posons,

$$
\begin{aligned}
\delta(g)= & \sup \quad \sup \quad \log \|M(d(k g) x)\| \\
& k \in K \quad x \in D \cap K \\
= & \sup \sup \log \left\|M\left(d\left(k_{g k}{ }^{-1}\right) x\right)\right\| \\
& k \in K \quad x \in D \cap K
\end{aligned}
$$

Nous avons $\delta(g k)=\delta(g)(g \in G, k \in K) \quad \delta$ est indépendante du choix de la section mesurable $\mathrm{s}$. Comme l'image de $K$ par la représentation adjointe de $G$ est compacte, $M(D \cap K)$ est un groupe compact de matrices et, pour chaque $g \in G,\left\{\mathrm{kgk}^{-1}, k \in K\right\}$ est un compact de $G$. Il s'ensuit que $\delta$ est une fonction continue de $G$ dans $\mathbb{R}_{+}$. [ La continuité résultant du fait que l'on sait aussi que (voir démonstration du lemme $(2.4$.$) ) si ( g_{n}$ ) est une suite d'éléments de $G$ convergeant vers $g=d k \in G$ avec $d \in D$ et $k \in K$, alors nous pouvons écrire $g_{n}=d_{n} k_{n}$ où $d_{n} \in D, k_{n} \in K$ sont respectivement des suites de $D$ et $K$ 
convergeant vers $d$ et $k$ ] .

$\delta$ définit alors une fonction continue sous additive de $G$; il est en effet facile de vérifier que l'on a

Nous avons

$$
\delta\left(g_{1} g_{2}\right) \leq \delta\left(g_{1}\right)+\delta\left(g_{2}\right) \quad\left(g_{1}, g_{2} \in G\right) .
$$

par suite

$$
D_{n}=D_{0} d\left(K_{0} d_{1}\right) \ldots d\left(K_{n-1} d_{n}\right) x_{n} \quad \text { avec } x_{n} \in D \cap K
$$

$$
M\left(D_{n}\right)=M\left(x_{n}\right) M\left(d\left(K_{n-1} d_{n}\right)\right) \ldots M\left(d\left(K_{0} d_{1}\right) M\left(D_{0}\right)\right.
$$

(comme $D$ opère à droite $J_{r}$, nous avons $M\left(d_{1} d_{2}\right)^{\prime}=M\left(d_{2}\right) M\left(d_{1}\right)\left(d_{1}, d_{2} \in D\right)$ ). $M\left(D_{n}\right)$ est donc le produit de $(n+2)$ matrices triangulaires supérieures. Pour $r \in \mathbb{N}$, nous avons

$$
\log \left\|M\left(d\left(K_{r} d_{r+1}\right)\right)\right\| \leq \delta\left(Y_{r+1}\right) .
$$

Or $\mu$ ayant un moment d'ordre 1 , nous avons $\delta_{G} \delta(g) \mu(d g)<+\infty((6.3)$.$) ;$ c'est-à-dire

$$
\sum_{r \geq 1} \mu(\{g \in G: \delta(g) \geq r \alpha\})<+\infty \quad \forall \alpha>0
$$

il s'ensuit que

$$
\sum_{r \geq 1} \mathbb{P}_{g}\left[\left\{\log \left\|M\left(d\left(K_{r} d_{r+1}\right)\right]\right\| \geq r \alpha\right\}\right]<+\infty \forall \alpha>0, \forall g \in G,
$$

qui implique

$$
\mathbb{P}_{g}\left[\lim { }_{r} \sup \left\|M\left(K_{r} d_{r+1}\right)\right\| \geq e^{r \alpha}\right]=0 \quad \forall \alpha>0, \forall g \in G,
$$

C'est-à-dire $\quad \lim _{r} \sup \left\|M\left(k_{r} d_{r+1}\right)\right\|^{1 / r} \leq 1 \quad \mathbb{P}_{g}-$ p.s. $\quad \forall g \in G$

Posons alors, pour $r \geq 0$,

$M\left(d\left(K_{r} d_{r+1}\right)\right)=\left[\frac{1}{0} \mid \frac{{ }_{r}}{Q_{r}}\right] ; \quad M\left(x_{r}\right)=\left[\frac{1}{0} \mid \frac{B\left(x_{r}\right)}{Q\left(x_{r}\right)}\right]$ et $M(D)=\left[\frac{1}{0} \mid \frac{Z_{r}}{\pi_{r}}\right]$

La suite des matrices $\left(Q_{r}\right), r \geq 0$, vérifie, $\mathbb{P}_{g}-p . s . \forall g \in G$, les hypothèses

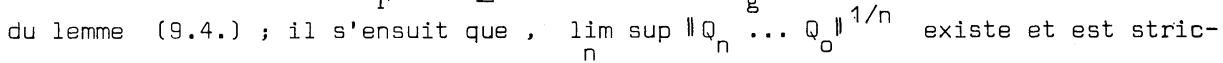
tement inférieure à $1, \mathbb{P}_{g}-p \cdot s . \forall g \in^{n} G$.

$M(D \cap K)$ étant compact, il existe un réel positif $C$ tel que

$$
\sup _{r \in \mathbb{N}}\left\|Q\left(x_{r}\right)\right\| \leq C \text { et } \sup _{r \in \mathbb{N}}\left\|B\left(x_{r}\right)\right\| \leq C
$$

Ii s'ensuit que : d'une part $\lim _{n}$ sup $\left\|_{\pi_{n}}\right\|^{1 / n}$ existe et est strictement inférieure à $1, \mathbb{P}_{g}-$ p.s. $\forall g \in G$; d'autre part, $z_{n}=z_{0}+B_{0} \pi_{0}+B_{1} Q_{0} \pi_{0}+\ldots+B_{n-1} Q_{n-2} \cdots Q_{0} \pi_{0}+B\left(x_{n}\right) Q_{n-1} \cdots Q_{0} \pi_{0}$ converge, $\mathbb{P}_{g}-$ p.s. $\forall g \in G$, vers une matrice $z_{\infty}$. 


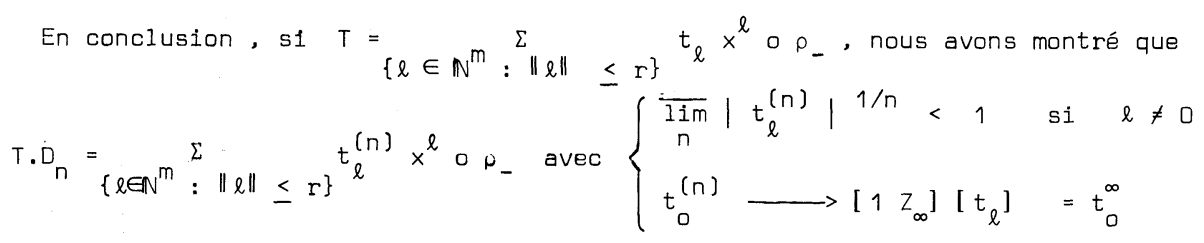

Si nous remarquons alors que pour tout $u \in h$,

$$
k \cdot \rho_{-}(u)=\left\{\exp _{G_{-}}^{-1} \circ \mu_{-}\left(k \exp u k^{-1}\right), k \in K\right\}
$$

est un compact de $\boldsymbol{h}_{-}$, on en déduit que

$$
T \cdot x_{n}(u)=T \cdot D_{n}\left(k_{n} \cdot u\right) \longrightarrow t_{0}^{\infty} \quad P_{g} \text {-p.s. } \quad \forall g \in G
$$

ce qui montre que pour tout $T \in J$, la suite de fonctions analytiques $T . X_{n}$, converge $\mathbb{P}_{g}$-p.s. $\forall g \in G$, vers une fonction constante (i.e. $\lim _{n} T . x_{n}(u)$ est indépendant de $u \in h$ ).

Le résultat cherché s'obtient alors en prenant pour $T$ les coordonnées $\left(x_{i} \circ \rho_{-}\right)_{1} \leq i \leq m$.

9.6. Preuve du ii) du théorème (9.2). - Nous reprenons les notations de (9.0) et (9.1). La démonstration de ii) étant analogue à celle de i), nous n'entrons pas dans les détails.

Le groupe $D_{+}^{*}=\exp \zeta_{+}^{*}$ est nilpotent simplement connexe; $D_{+}^{*}$ est donc isomorphe au groupe $\left(\xi_{+}^{*}, x\right) \cdot h_{+}^{*}$ apparait alors comme un D-espace homogène à droite D-homéomorphe à $D_{-} D_{0} \backslash^{D}$; l'action de $D$ sur $h_{+}^{*}$ étant définie par

$$
u \cdot d=\exp _{D_{+}^{*}}^{-1} \text { o } p_{+}^{*}((\exp u) d) \quad\left(u \in \eta_{+}^{*}, d \in D\right)
$$

Etendons cette définition à $G$ en posant

$$
u \cdot g=\exp ^{-1} ._{+}^{*} \circ{p_{+}^{*}}_{+}^{*}((\exp u) g) \quad\left(u \in h_{+}^{*}, g \in G\right)
$$

On vérifie que l'on a

$$
\text { (*) } u .(d g)=(u \cdot d) \cdot g \quad(d \in D, g \in G) \text {. }
$$

Considérons l'ensemble $\mathcal{J}$ ' des fonctions polynomiales sur $h$, à valeurs complexes, qui sont invariantes à gauche par $h-\otimes h_{0}$ (i.e. $T \in J$ ' si $T \circ L_{C}=T$, $\forall \dot{c} \in h_{-} \boxplus h_{0}(\operatorname{voir} \& 3)$. J' est l'ensemble des fonctions polynomiales sur $h$ de la forme $T^{\prime} \circ \rho_{+}^{*}$, où $T^{\prime}$ est une fonction polynomiale sur

$\zeta_{+}^{*}$.

Pour $T \in J$ ' et $g \in G$, on définit

$$
\text { g.T }: u \in h \longrightarrow T\left(\rho_{+}^{*}(u) \cdot g\right)
$$

De (*) il résulte que $l$ 'on a $(d g) . T=d .(g . T)(d \in D, g \in G)$. 
En outre, si $d=(\exp v) x \in D$ avec $v \in \mathcal{h}$ et $x \in P A$, on vérifie que

$$
\text { (**) } \quad d . T=T \circ A d x^{-1} \circ R_{v}
$$

Pour tout entier naturel $r$, appelons J' $r$ le sous-espace vectoriel de J' formé des éléments de degré inférieur ou égal à $r$; J' et les sous-espaces $J^{\prime} r, r \in \mathbb{N}$, sont alors des $D$-espaces à gauche. Choisissons une base $\left(e_{i}\right)_{1} \leq i \leq m$ de $\left(h_{+}^{*}\right)^{\mathbb{C}}$ qui triangularise inférieure-

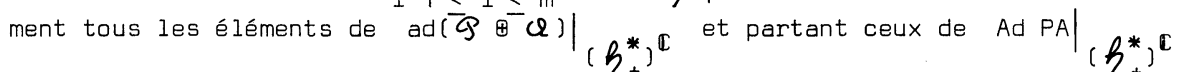
Si $x \in P A$, dans cette base, Adx est une matrice triangulaire inférieure dont les éléments diagonaux sont $\left\{\phi_{\lambda}(x), \lambda \in \Xi_{+}^{*}\right\}$.

Soit $\left(x_{i}\right)_{1}<i<m_{*}$ le système de fonctions coordonnées associé à cette base de $\left(\zeta_{+}^{*}\right)^{\mathbb{C}}$. Pour ${ }_{r} \in \mathbb{N}^{*}$ nous choisissons pour base de $J^{\prime} \mathrm{r}$ la base ordonnée $\left\{1, x^{\ell} \circ \rho_{+}^{*}, \ell \in \mathbb{N}^{m}-\{0\}\right.$, $\left.\|\ell\| \leq r\right\} \quad$ (voir (9.3.)). Il est clair que dans cette base l'action d'un élément d de $D$ est représenté par une matrice $M^{\prime}(d)$ triangulaire supérieure ayant 1 puis des produits $\phi_{\lambda_{1}}\left(d^{-1}\right) \ldots \phi_{\lambda_{s}}\left(d^{-1}\right)$, où $\lambda_{1}, \ldots, \lambda_{s} \in \Xi_{+}^{*}$ pour éléments diagonaux. $\left[\phi_{\lambda_{1}}\left(d^{-1}\right) \ldots \phi_{\lambda_{s}}\left(d^{-1}\right)\right.$ et non $\phi_{\lambda_{1}}(d) \ldots \phi_{\lambda_{S}}(d)$ car dans $(* *)$ c'est Adx $^{-1}$ qui intervient et non Adx] .

Si on écrit alors $x_{n}=D_{n} K_{n}$ avec $D_{n} \in D$ et $K_{n} \in K$ (voir (9.5.)) $M^{\prime}\left(D_{n}\right)$ est une matrice triangulaire supérieure dont les éléments diagonaux sont 1 puis des produits de la forme $\phi_{\lambda_{1}}\left(D_{n}^{-1}\right) \ldots \phi_{\lambda_{s}}\left(D_{n}^{-1}\right)$, où $\lambda_{1}, \ldots, \lambda_{s} \in \Xi_{+}^{*}$.
Or si $\lambda \in \Xi_{+}^{*}$, on a

$$
\log \left|\phi_{\lambda}\left(D_{n}^{-1}\right)\right|=-\left.\log \right|_{\lambda}\left(D_{n}\right) \mid=-\tau_{\lambda}\left(v_{0}, x_{n}\right) \longrightarrow-a_{\lambda}<0 .
$$

Par suite, les éléments diagonaux de $M^{\prime}\left(D_{n}\right)$, autres que 1 , convergent exponentiellement vers zéro. La fin de la preuve de ii) est alors claire.

D'après le théorème (9.2.) nous savons que pour tout $g \in G$, la composante $\mu_{-}\left(X_{n} g\right)$, de $x_{n} g$ sur $G_{-}$converge $\mathbb{P}_{x}-$ p.s. $\forall x \in G$. Il s'ensuit que pour tout $h \in G$ et tout $g \in G$ la composante, $\mu_{-}\left(h x_{n} g\right)$, de $h x_{n} g$ sur $G_{-}$converge $\mathbb{P}_{x}$ - p.s. $\forall x \in G$. Nous avons alors :

9.7. Théorème. - Soient (G, $\mu$ ) comme dans le théorème (9.2.) . Considérons les décompositions $G=G_{-}^{\mu} E_{+}^{\mu}$ et $G=G_{-}^{\mu} G_{+}^{\mu}$ modulo $m_{G}$, associées à $\mu$ (voir (7.17.)). Pour tout $g \in G$, nous savons (théorème (9.2.)) que Za composante de $\mathrm{gX}_{\mathrm{n}}$ sur $\mathrm{G}_{-}^{\mu}$ dans la décomposition $\mathrm{G}=\mathrm{G}_{-}^{\mu} \mathrm{E}_{+}^{\mu}$ converge, $\mathbb{P}_{\mathrm{X}}$ - p.s. $\forall x \in G$, vers une variable aléatoire $\mathrm{Z}(\mathrm{g},$.$) à valeurs dans \mathrm{G}_{-}^{\mu}$. Si on identifie $G_{-}^{\mu}$ à un ouvert de ${ }^{G}{ }_{G_{+}^{\mu}}$, alors nous avons

$$
Z(g, .)=g \cdot Z(e, .) \quad \mathbb{P}_{x}-p \cdot s . \quad \forall x \in G .
$$


Pour prouver le théorème (9.7.), nous commençons par établir la proposition :

9.8. Proposition. - Soit (G, $\mu$ ) comme dans le théorème (9.2.). Soient

$G=G_{-}^{\mu} E_{+}^{\mu}$ une décomposition de $G$ associée à $\mu$ (voir (7.17.)) et

$\left(\Omega, G_{F},\left(X_{n}\right)_{n} \in \mathbb{N},\left(\mathbb{P}_{x}\right)_{x} \in \underline{G}\right)$ la marche aléatoire droite de Zoi $\mu$ sur $G$. Ecrivons $X_{n}=X_{n}^{-} X_{n}^{+}$avec $X_{n}^{-} \in G_{-}^{\mu}, X_{n}^{+} \in E_{+}^{\mu}$. Alors $i l$ existe un sous-ensemble mesurable $\Omega^{\prime}$ de $\Omega$, de $\mathbb{P}_{x}$-mesure 1 pour tout élément $x$ de $G$, sur lequel $p_{-}\left(u x_{n}^{+}\right), u \in \tilde{N}$, converge uniformément sur tout compact de $\tilde{N}$ vers l'élément neutre e de $\mathrm{G}$.

Nous reprenons les notations de $(9.5$.$) et nous désignons par A_{n}$ la composante de $D_{n}=d\left(X_{n}\right)$ sur $A$ dans la décomposition $D=$ RNA (voir lemme (1.5.)). Posons

$\Omega_{1}=\left\{\omega \in \Omega: \frac{1}{n} \tau_{\lambda}\left(v_{0}, x_{n}\right) \longrightarrow a_{\lambda}, \forall \lambda \in \Xi_{-}\right\} ;$

$\Omega_{1}$ est de $\mathbb{P}_{x}$-mesure 1 , pour tout $x$ de $G$, et nous avons

9.9. Lemme. - Pour tout $\omega$ de $\Omega_{1}, A_{n}^{-1}(\omega) \cup A_{n}(\omega), u \in \tilde{N}$, converge uniformément sur tout compact de $\tilde{N}$ vers $e$.

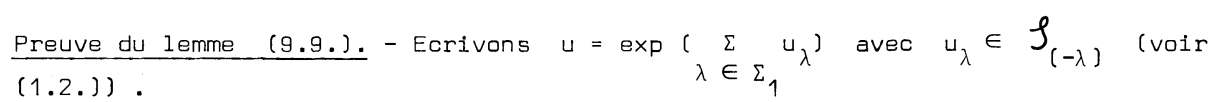
Nous avons :

$$
\begin{aligned}
A_{n}^{-1} \cup A_{n} & =\exp \left(\sum_{\lambda \in \Sigma_{1}} \operatorname{Ad~} A_{n}^{-1}\left(\left(u_{\lambda}\right)\right)\right. \\
& =\exp \left(\sum_{\lambda \in \Sigma_{1}}\left|\phi_{\lambda}\left(A_{n}^{-1}\right)\right|^{-1} u_{\lambda}\right) \\
& =\exp \left(\sum_{\lambda \in \Sigma_{1}} \phi_{\lambda}\left(A_{n}\right) u_{\lambda}\right)
\end{aligned}
$$

Or sur. $\Omega_{1}, \frac{1}{n} \log \left|\phi_{\lambda}\left(A_{n}\right)\right|=\frac{1}{n} \tau_{\lambda}\left(v_{0}, x_{n}\right)$ converge vers $a_{\lambda}<0$, car $\lambda \in \Sigma_{1} \subset \Xi_{-}$. Le lemme (9.9.) est donc prouvé.

9.10. Preuve de la proposition (9.8.). - Reprenons les notations de (9.0.) et (9.1.).

Nous avons

$$
h=h_{-} \rightarrow h_{+} \text {avec } h_{-}=\mu_{-} \boxplus \mathcal{N} \text { et } h_{+}=\mu_{+} \text {. }
$$

Nous savons que $\mu_{+}$se décompose en somme directe de deux sous-algèbres $\mu_{0}$ et $\mu_{+}^{*}$ telles que $D_{+}$soit le produit amalgamé des sous-groupes $D_{0}=\left(\exp \mu_{0}\right) P A$ et $D_{+}^{*}=\exp \mu_{+}^{*}$; en outre $D_{0}$ normalise $D_{-}$et $D_{+}^{*}$. Si 
d est un élément de $D$, nous notons respectivement $p_{-}(d), p_{0}(d)$ et $p_{+}^{*}(d)$ les éléments de $D_{-}, D_{0}$ et $D_{+}^{*}$ tels que $d=\mu_{-}(d) p_{0}(d) \mu_{+}^{*}(d)$.

Avec les notations de $(9.5$.$) , posons d^{0}(g)=\mu_{0}(d(g))$; nous avons

$\mu_{0}\left(D_{n}\right)=d^{0}\left(x_{n}\right)=d^{0}\left(x_{n}^{+}\right)=d^{0}\left(D_{0}\right) d^{0}\left(\mu_{+}^{*}\left(D_{0}\right) k_{0} d_{1}\right) \ldots d^{0}\left(\mu_{+}^{*}\left(D_{n-1}\right) k_{n-1}{ }_{n}\right)$

$(\bmod . D \cap K)$

Soit $C$ un compact de $\tilde{N}$. Pour tout élément $u$ de $C$, posons $v_{p}(u)=\left(A_{p-1}^{-1} \cup A_{p-1} d^{0} \cdot\left(p_{+}^{*}\left(D_{p-1}\right) k_{p-1} d_{p}\right)\left(A_{p}^{-1} u^{-1} A_{p}\right)\right.$

et

$v_{0}(u)=u d^{0}\left(D_{0}\right)\left(A_{0}^{-1} u^{-1} A_{0}\right)$

Nous avons alors $v_{p} \in D_{+}, 0 \leq P \leq n$ et

$u \mu_{0}\left(D_{n}\right)=v_{0} \cdots v_{n}\left(A_{n}^{-1} u A_{n}\right) \bmod \cdot(D \cap K)$.

[ En effet : la deuxième assertion est évidente; quant à la première, si nous écrivons

$d^{0}\left(\mu_{+}^{*}\left(D_{p-1}\right) K_{p-1} d_{p}\right)=r_{p} a_{p}$ avec $r_{p} \in\left(\exp \mu_{0}\right) p$ et $a_{p} \in A$

Nous avons

$v_{p}=\left(\left[A_{p-1}^{-1} \cup A_{p-1}\right] \quad r_{p}\left[A_{p-1}^{-1} u^{-1} A_{p-1}\right]\right) a_{p}\left(\operatorname{car} A_{p}=A_{p-1} a_{p}\right) ;$ Comme $\left[\tilde{\mathcal{N}}, \mu_{+}\right] \subset \mu_{+}$et $[\mathscr{S}, \mathcal{S}]=(0)$, on en déduit que $\left.v_{p} \in D_{+}\right]$

L'action de $v_{n}=v_{0} \ldots v_{n} \in D_{+}$sur $J_{r}$ est représentée par la matrice $M\left(V_{n}\right)$

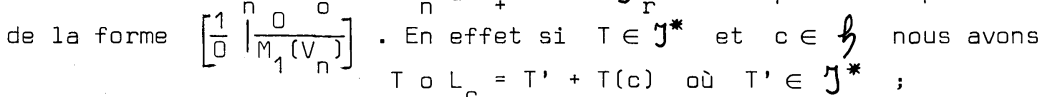

par suite si $c \in h_{+}$, nous avons $T(c)=T(0)=0$; autrement dit $T \circ L_{c} \in J^{*}$.

Pour tous éléments d et $h$ respectivement de $D$ et exp $h$, les matrices $M(d)$ et $M(d h)$ ont les mêmes éléments diagonaux. Il s'ensuit que, pour tout $p \in\{0, \ldots, n\}$, les éléments diagonaux de $M\left(v_{p}\right)$ sont ceux de $M\left(d\left(p^{*}\left(D_{p-1}\right) K_{p-1} d_{p}\right)\right)$.

Par suite, la diagonale de la matrice $M\left(V_{n}\right)$ est le produit par la diagonale d'un élément de $M(D \cap K)$ de la diagonale de la matrice $M\left(D_{n}\right)$. $M_{1}\left(V_{n}\right)$ est donc une matrice triangulaire supérieure dont les racines enièmes des modules des éléments diagonaux sont indépendants de $u$ et convergent sur $\Omega_{1}$ vers des éléments strictement inférieure à 1 (voir (9.5.)) .

Admettons un instant que nous ayons montré qu'il existe un sous ensemble $\Omega_{2}$ de $\Omega_{1}$, de $\mathbb{P}_{x}$-mesure 1 pour tout élément $x$ de $G$, sur lequel on ait

$\lim _{p}\left(\sup _{u \in C}\left(\left\|M\left(v_{p}(u)\right)\right\|\right)\right)^{1 / p} \leq 1$, pour tout compact $C$ de $\tilde{N}$.

La preuve du lemme (9.4.) nous montre alors que sur $\Omega_{2}$

$\overline{\lim }\left(\sup _{n}\left(\left\|M_{1}\left(V_{n}(u)\right)\right\|\right)\right)^{1 / n}$ existe et est strictement inférieure à 1 , pour 


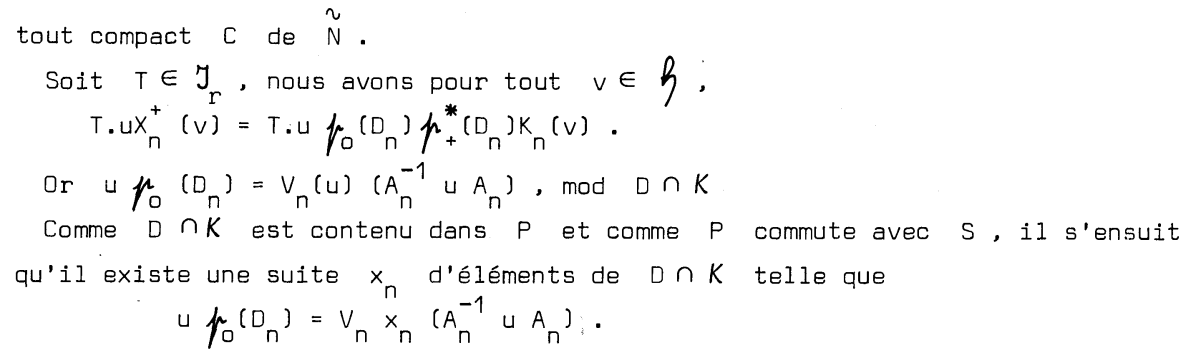

D' où

$$
\begin{aligned}
T . u x_{n}^{+}(v) & =T \cdot v_{n} x_{n}\left(A_{n}^{-1} \cup A_{n}\right) \mu_{+}^{*}\left(D_{n}\right) k_{n}(v) \\
& =M\left(x_{n}\right) M\left(v_{n}\right) T\left(\left(A_{n}^{-1} \cup A_{n}\right) \mu_{+}^{*}\left(D_{n}\right) K_{n} \cdot \rho_{-}(v)\right)
\end{aligned}
$$

Posons alors

$\Omega^{\prime}=\left\{\omega \in \Omega_{1}: \lim \mu_{+}^{*}\left(D_{n}\right)\right.$ existe $\cap_{n} \Omega_{2}$.

$\Omega^{\prime}$ est de $\mathbb{P}_{x}$-mesure ${ }_{\sim}$, pour tout élément $x$ de $G$. Soit $\omega \in \Omega^{\prime}$ et $C$ un compact quelconque de $\tilde{N}_{-1}$. Comme $K_{.} \rho_{-}(v)$ est compact, comme $h_{+}^{*}\left(D_{n}(\omega)\right)$ converge et comme $A_{u}^{-1}(\omega) \cup A_{n}(\omega)$ converge vers e uniformément sur $c$ (lemme $(9.9)$.$) ,$

$$
\left(A_{n}^{-1}(\omega) \cup A_{n}(\omega)\right) \mu_{+}^{*}\left(D_{n}(\omega)\right) K_{n}(\omega) \cdot p_{-}(v),
$$

reste dans un compact de $h_{-}$- quand $u$ décrit $c$ et $n$ décrit $\mathbb{N}$. Il s'ensuit donc que pour tout $v \in h^{-}$et tout $\omega \in \Omega, T . u X_{n}^{+}(\omega)(v)$ converge uniformément sur $C$ vers $T(0)$.

En prenant pour $T$ les coordonnées $\left(x_{i} \circ \rho_{-}\right), 1 \leq i \leq m$, on en déduit que pour tout $\omega \in \Omega^{\prime}$, la composante sur $G_{-}$de $u X_{n}^{+}(\omega)$ converge uniformément sur C sur e.

Pour terminer la preuve de la proposition (9.8.) on est donc amené à prouver qu'il existe un sous-ensemble $\Omega_{2}$ de $\Omega_{1}$, de $\mathbb{P}_{x}$-mesure 1 pour tout élément $x$ de $G$, sur lequel on ait

$$
\overline{\operatorname{Iim}}_{P}\left(\sup _{u \in C}\left(\left\|M\left(v_{p}(u)\right)\right\|\right)\right)^{1 / p} \leq 1 \text {, }
$$

pour tout compact $C$ de $\tilde{N}$.

Soit $r$ un entier naturel supérieur ou égal à la longueur $p$ de la série centrale descendante de l'algèbre de Lie nilopotente $\boldsymbol{h}$. Si d est un élément de $D$, on note $M(d)$ (resp. $M^{\prime}(d)$ ) l'action de d sur l'espace vectoriel $J_{r}$ (resp $J^{\prime}{ }_{r}$ ) des polynomes, de degré inférieur ou égal à $r$, invariants à droite par $h_{+}{ }^{r}$ (resp. invariants à gauche par $h_{-} \oplus h_{0}$ ).

9.11. Lemme. - Il existe des réels positifs $\alpha$ et $\beta$ tels que pour tout $d \in D$,

$\left\|M\left(\mu_{0}(d)\right)\right\|<\beta \quad(\sup \{1,\|M(d)\|,\|M \cdot(d)\|\})^{\alpha}$ 
Preuve du lemme (9.11.). - Si $r$ est un entier positif supérieur ou égal à longueur $p$ de la série centrale de $h$, nous avons $\left[p_{-}(d)\right]^{-1}=\exp \left(-\sum_{i=1}^{m}\left[M(d)\left(x_{i} \circ p_{-}\right)\right](0) \cdot e_{i}\right)$.

où $\left(e_{i}\right)_{1 \leq i \leq m}$ désigne la base de $h_{-}^{\mathbb{C}}$ considérée en $(9.3$.$) ; et de même$ $\left[h_{+}^{*}(d)\right]^{-1}=\exp \left(-\sum_{i=1}^{m}\left[M^{\prime}(d)\left(x_{i} \quad 0 \quad \rho_{-}\right)\right](0) \cdot e_{i}\right)$,

○ப̀ $\left(e_{i}\right)_{1 \leq i \leq m}$ désigne la base de $\left(h_{+}^{*}\right)^{\mathbb{C}}$ considérée en (9.6.). Nous avons $D_{-}=\exp h_{-}$et $D_{+}^{*}=\exp h_{+}^{*} ;$ vue la façon dont $\exp h$ agit sur $I_{r}(v o i r$ formule $(* *)$ de $(9.3)$.$) , il est alors clair qu'il existe deux$ constantes positives $\alpha^{\prime}$ et $\beta^{\prime}$ telles que

$$
\begin{aligned}
& \left\|M\left(p_{-}(d)\right)^{-1}\right\|<\beta^{\prime} \quad(\sup \{1,\|M(d)\|\}\}^{\alpha^{\prime}} \\
& \left\|M\left(h_{+}^{*}(d)\right)^{-1}\right\|<\beta^{\prime}(\sup \{1,\|M(d)\|\})^{\alpha^{\prime}}
\end{aligned}
$$

Le lemme (9.11.) résulte alors de l'inégalité, $\left\|M\left(\mu_{0}(d)\right)\right\|<\left\|M\left[\left(\mu_{-}(d)\right)^{-1}\right]\right\|\|M(d)\|\left\|M\left[\left(\mu_{+}^{*}(d)\right)^{-1}\right]\right\|$.

$$
\begin{aligned}
& \text { Posons } \\
& \delta_{1}(g)=\sup _{k \in K} \sup _{x \in D \cap K}(\|M(d(k g) x)\|)
\end{aligned}
$$
et

$$
\delta_{2}(g)=\sup _{k \in K} \sup _{x \in D \cap K}^{(\|M \prime(d(k g) x)\|)}
$$

$\delta_{1}$ et $\delta_{2}$ sont alors deux fonctions sous-multiplicatives continues positives sur $G$ (i.e. $\delta\left(g_{1} g_{2}\right) \leq \delta\left(g_{1}\right) \delta\left(g_{2}\right)$, pour $\left.g_{1}, g_{2} \in G\right)$.

D'autre part, posons pour $y \in P A$ $\sigma(y)=\inf \{\|M(h y)\|: h \in P A \cap \exp h\} ;$ puis pour $d=(\exp m) y \in D$ avec $m \in h$ et $y \in P A$, $\sigma(d)=\sigma(y)$

Comme $\exp h$ est distingué dans $D$, on définit ainsi une fonction multiplicative continue positive sur $D$ telle que

En posant alors

$$
\sigma(h d)=\sigma(d) \quad(d \in D, h \in \exp h)
$$

$$
\delta_{3}(g)=\sup _{k \in K} \sup _{x \in D \cap K^{\sigma(d(k g) x)},}
$$

on obtient une fonction sous-multiplicative continue positive de $G$.

$\mu$ possède un moment d'ordre 1 . Il s'ensuit que l'on a, $\mathbb{P}_{x}-p . s . \forall x \in G$, $\overline{\lim }_{p} \delta_{i}\left(Y_{p}\right)^{1 / p} \leq 1 \quad i \in\{1,2,3\}$, 
où les $Y_{i}, i \geqslant 1$, sont des v.a. indépendantes de loi $\mu$.

Posons

$\Omega_{2}=\left\{\omega \in \Omega_{1}: \overline{\lim }_{p} \delta_{i}\left(Y_{p}(\omega)\right\}^{1 / p} \leq 1, i \in\{1,2,3\}\right.$, et $\overline{\lim } h_{+}^{*}\left(D_{p}(\omega)\right)$ existe \}

$\Omega_{2}$ est de $\mathbb{P}_{x}$-mesure 1 pour tout élément $x$ de $G$. Sur $\Omega_{2}$ nous avons

i) $\overline{\operatorname{Iim}}\left\|M\left(d\left(K_{p-1} d_{p}\right)\right)\right\|<\overline{\lim } \delta_{1}\left(Y_{p}\right)^{1 / p} \leq 1$

ii) $\overline{\operatorname{Iim}}\left\|M \cdot\left(d\left(K_{p-1} d_{p}\right)\right)\right\|<\overline{\lim }_{p} \delta_{2}\left(Y_{p}\right)^{1 / p} \leq 1$

iii) $\overline{\lim }_{p} \sigma\left(d\left(k_{p-1} d_{p}\right)\right)^{1 / p}<\overline{\lim }_{p} \delta_{3}\left(Y_{p}\right)^{1 / p} \leq 1$

De i), ii) et du lemme (9.11.), il résulte que sur $\Omega_{2}$ on a

$$
\overline{\operatorname{Iim}}_{\mathrm{p}}\left\|M\left(d^{0}\left(p_{+}^{*}\left(D_{p-1}\right) k_{p-1} d_{p}\right)\right)\right\| \leq 1
$$

D'autre part, de il et iiil il résulte que sur $\Omega_{2}$,

$d\left(k_{p-1} d_{p}\right)$ s'écrit sous la forme $h_{p} y_{p}$ où $h_{p}$ et $y_{p}$ sont respectivement des suites d'éléments de $\exp h$ et $P A$ telles que

$$
\overline{\lim }\left\|M\left(h_{p} y_{p}\right)\right\|^{1 / p} \leq 1 \text { et } \overline{\lim }_{p}\left\|M\left(y_{p}\right)\right\|^{1 / p} \leq 1
$$

Mais comme $M\left(y_{p}^{-1}\right)=\left(M\left(y_{p}\right)\right)^{-1}$ on a aussi

$\overline{\lim }_{p}\left\|M\left(y_{p}^{-1}\right)\right\| \leq 1$

On en déduit que sur $\Omega_{2}$, $d^{0}\left(\mu_{+}^{*}\left(D_{p-1}\right) k_{p-1} d_{p}\right)$ s'écrit (exp $\left.m_{p}\right) y_{p}$, où $m_{p}$ et $y_{p}$ sont respectivement des suites d'éléments de $\mu_{0}$ et $P{ }^{P}$ telles que $\underset{p}{\lim }\left\|M\left(\exp m_{p}\right)\right\|^{1 / p} \leq 1$ et $\overline{\lim }\left\|M\left(y_{p}\right)\right\|^{1 / p} \leq 1$. Nous avons alors

$$
v_{p}(u)=\left(\exp m_{p}^{\prime}(u) y_{p}\right) \text {, }
$$

où $m_{p}^{\prime}(u)=\left[\operatorname{Ad}\left(\left(A_{p-1}^{-1} \cup A_{p-1}\right)\right]\left(m_{p}\right)\right.$.

Comme sur $\Omega_{1}, A_{p-1}^{-1} \cup A_{p-1}$ converge uniformément sur $C$ vers $e$ (lemme (9.9.)), il est alors clair, vue la façon dont exp $h$ agit sur $J_{r}$, (voir formule $(* *)$ de (9.3.)) que l'on a sur $\Omega_{2}$,

et par suite

$$
\left.\overline{\lim } \underset{\mathrm{P}}{ } \sup _{u \in C}\left\|M\left(m^{\prime}(u)\right)\right\|\right)^{1 / p} \leq 1
$$

$$
\left.\overline{\lim } \sup _{u \in C}\left\|M\left(v_{p}(u)\right)\right\|\right)^{1 / p} \leq 1
$$

La preuve de la proposition (9.8.) est achevée.

9.12. Lemme. - Pour tout $\dot{g} \in \mathrm{G}$, nous avons

$$
g Z(e, .) \in G_{-} G_{+}, \mathbb{P}_{x} \text {-p.s. } \forall_{x} \in G \text {, }
$$


Preuve. - $\mu$ étant étalée, pour $n$ assez grand nous avons
(*) $\mu^{n}=f_{n} \cdot m_{G}+v_{n}$
avec $\left\|v_{n}\right\| \leqslant \rho^{n}$
$0 \leqslant \rho \leqslant 1$

où $f_{n} \in L^{1}\left(m_{G}\right)$ et $\nu_{n}$ est une mesure étrangère par rapport à $m_{G}$.

Pour tout entier $P$ il est facile de voir que l'on a

$\mathbb{P}_{x}\left[g Z(e,.] \notin G_{-} G_{+}\right]=\iiint_{g}^{-1}\left(G_{-G_{+}} c^{\left(Z\left(X_{p}(\omega), \omega^{\prime}\right)\right) d \mathbb{P}_{x}(\omega) d \mathbb{P} e\left(\omega^{\prime}\right) .}\right.$

Soit

$$
B=\left\{\left(\omega, \omega^{\prime}\right) \in \Omega \times \Omega \quad: \quad X_{p}(\omega) Z\left(e, \omega^{\prime}\right) \in G_{-} \dot{G}_{+}\right\} ;
$$

nous avons, pour $p$ assez grand,

$$
\begin{aligned}
\int{ }_{B^{C}}\left(\omega, \omega^{\prime}\right) d \mathbb{P}_{x}(\omega) & =\varepsilon_{x} * \mu^{P}\left(\left(G_{-} G_{+}\right)^{C}\left(Z\left(e, \omega^{\prime}\right)\right)^{-1}\right) \\
& =\varepsilon_{x} * \nu_{P}\left(\left(G_{-} G_{+}\right)^{C}\left(Z\left(e, \omega^{\prime}\right)\right)^{-1}\right) \\
& \leqslant \rho^{P} ;
\end{aligned}
$$

d' où

$\mathbb{P}_{x}\left[g Z(e,.) \notin G_{-} G_{+}\right] \leqslant \rho^{p}+\iint 1_{B}\left(\omega, \omega^{\prime}\right) 1_{g^{-1}\left(G_{-} G_{+}\right)^{C}}\left(Z\left(x_{p}(\omega), \omega^{\prime}\right)\right) d \mathbb{P}_{x}(\omega) d \mathbb{P}_{e}\left(\omega^{\prime}\right)$

Soit $\Omega$ ' le sous-ensemble de $\Omega$ intervenant dans la proposition (9.8.).

$D^{\prime}$ après cette proposition, il est clair que pour tout $\left(\omega, \omega^{\prime}\right) \in B \cap\left(\Omega \times \Omega^{\prime}\right)$, $\lim _{n} \mu_{-}\left(x_{p}(\omega) Z\left(e, \omega^{\prime}\right) x_{n}^{+}\left(\omega^{\prime}\right)\right)$ converge vers la composante sur $G_{-}$de $X_{p}(\omega) Z\left(e, \omega^{\prime}\right)$ dans la décomposition $G_{-} G_{+}$. D'autre part pour tout $\omega \in \Omega$, $\lim _{n} p_{-}\left(x_{p}(\omega) x_{n}().\right)$ existe et vaut $z\left(x_{p}(\omega),.\right) \mathbb{P}_{x}-p . s$. Nous pouvons done supposer, quitte à remplacer $B$ par un sous-ensemble mesurable de $\mathbb{P}_{X} \otimes \mathbb{P}_{e}$-mesure égale, que pour tout $\left(\omega, \omega^{\prime}\right) \in B$

i) $\lim \mu_{-}\left(x_{p}(\omega) x_{n}\left(\omega^{\prime}\right)\right)$ existe et vaut $z\left(x_{p}(\omega), \omega^{\prime}\right)$

ii) $\lim _{n} \mu_{-}\left(x_{p}(\omega) Z\left(e, \omega^{\prime}\right) X_{n}^{+}\left(\omega^{\prime}\right)\right)$ existe et est égale à la composante sur $G_{-}$ de $X_{p}(\omega) Z\left(e, \omega^{\prime}\right)$ dans la décomposition $G_{-} G_{+}$. Mais ces limites sont alors nécessairement égales et on en déduit que 1 'on a :

$$
\begin{aligned}
& \iint 1_{B}\left(\omega, \omega^{\prime}\right) 1 g^{-1}\left(G_{-} G_{+}\right)^{C}\left(Z\left(X_{p}(\omega), \omega^{\prime}\right)\right) d \mathbb{P}_{x}(\omega) d \mathbb{P}_{e}\left(\omega^{\prime}\right) \\
& =\iint 1_{B}\left(\omega, \omega^{\prime}\right) 1\left(g^{-1}\left(G_{-} G_{+}\right)^{c} \cap G_{-}\right) G_{+}\left(X_{P}(\omega) Z\left(e, \omega^{\prime}\right)\right) d \mathbb{P}_{x}(\omega) d \mathbb{P}_{e}\left(\omega^{\prime}\right) \\
& \leqslant \varepsilon_{x} * \mu^{P} * \lambda_{e}\left[\left(g^{-1}\left(G_{-} G_{+}\right)^{C} \cap G_{-}\right) G_{+}\right] \text {, } \\
& \text { où } \lambda_{e} \text { désigne la loi de la v.a. } Z(e, .) \text { par rapport à la probabilité } \mathbb{P}_{e} \\
& \leqslant \varepsilon_{x} * \mu^{P} * \lambda_{e}\left[G_{-} G_{+} \cap g^{-1}\left(G_{-} G_{+}\right]^{C}\right] \\
& \leqslant \varepsilon_{x} * \nu_{p} * \lambda_{e}\left[G_{-} G_{+} \cap g^{-1}\left(G_{-} G_{+}\right)^{c}\right] \\
& \leqslant \rho^{P}
\end{aligned}
$$


D'où $\mathbb{P}_{X}\left[g Z(e,.) \notin G_{-} G_{+}\right] \leq 2 \rho^{p}$, pour tout $p$ assez grand; autrement dit nous avons $\mathbb{P}_{x}\left[g Z(e,.) \notin G_{-} G_{+}\right]=0$ et le lemme $(9.12$.$) est prouvé.$

9.13. Lemme.- Pour tout gie G,

$G(g)=\left\{y \in G: g \quad \mu_{-}(y) \notin G_{-} G_{+}\right\}$

est de mesure de Haar nuzze.

Preuve. - Comme pour la démonstration de la proposition (5.4.), on se ramène à prouver le lemme dans le cas d'un groupe de Lie connexe semi-simple de centre fini. Dans ce cas la décomposition $G=G_{-\sim^{+}} n^{\prime}$ 'est autre que la décomposition de Bruhat $G=$ NAN $\tilde{N}$ avec $G_{-}=N$ et $G_{+}=\tilde{A N \Gamma}$ (voir prop. (5.3.)) )

Posons alors $N(g)=\{u \in N$ : gu $\notin N A N \Gamma\}$. Nous avons $G(g)=N(g) A K$. Pour montrer que $G(g)$ est de mesure de Haar nulle, on est donc amené à prouver que $N(g)$ est de mesure de Haar nulle dans $N$.

D'après le lemme de Bruhat, l'application $(n, a, \tilde{n}, \gamma) \longmapsto$ nañ est un isomorphisme de variétés analytiques de $N \times A \times N \times \Gamma$ sur un ouvert de $G$ dont

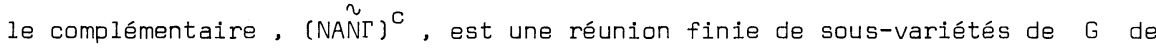
dimensions strictement plus petites. On en déduit que pour tout $g \in G$, $\left(\right.$ gNANT $^{C}=g(N A N \Gamma)^{C}$ est une réunion finie de sous variétés de $G$ de dimensions strictement plus petites.

Or nous avons

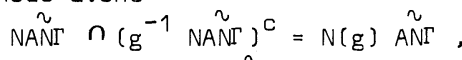

il s'ensuit que $N(g) A N \Gamma$ est de mesure de Haar nulle dans $G$; et par suite $N(g)$ est de mesure de Haar nulle dans $N$. Le lemme (9.13.) est prouvé.

9.14. Preuve du théorème (9.7.). - Soit $x \in G$. Ecrivons $x_{n}=x_{n}^{-} x_{n}^{+}$avec $x_{n}^{-} \in G_{-}$et $x_{n}^{+} \in E_{+}$. Nous avons, (pour $n$ assez grand),

$$
\mathbb{P}_{X}\left[g X_{n}^{-} \notin G_{-} G_{+}\right]=\mathbb{P}_{x}\left[X_{n} \in G(g)\right] \quad(x, g \in G)
$$$$
=\dot{\varepsilon}_{x} * \mu^{n}(G(g))
$$$$
=\varepsilon_{x} * \nu_{n}(G(g)) \quad(\text { lemme }(9.13 .)
$$

Par suite $\sum_{n \geqslant 0} \mathbb{P}_{x}\left[g X_{n}^{-1} \notin \rho_{-}^{n} \cdot G_{+}\right]<+\infty$ et d'après le lemme de Borel-Cantelli, il existe un sous-ensemble $\Omega^{\prime}{ }_{\mathrm{g}}, \mathrm{x}$ de $\Omega$ de $\mathbb{P}_{\mathrm{X}}$-mesure 1 tel que $\forall \omega \in \Omega^{\prime}{ }_{g}, x^{\prime}, \exists n_{0}(\omega) \in \mathbb{N}, \quad \forall n \geqslant n_{0}(\omega) \quad g x_{n}^{-}(\omega) \in G_{-} G_{+}$.

En tenant compte du lemme (9.12.), il s'ensuit qu'il existe, pour tout couple 
$(g, x) \in G \times G$, un sous-ensemble mesurable $\Omega_{g}, x$ de $\mathbb{P}_{x}$-mesure 1 tel que pour tout $\omega$ de $\Omega_{g}, x$,

i) $\exists n_{0}(\omega) \in \mathbb{N}, \forall n \geq n_{0}(\omega) \quad g x_{n}^{-}(\omega) \in G_{-} G_{+}$

ii) $\lim _{n} x_{n}^{-}(\omega)=Z(e, \omega)$ et $g Z(e, \omega) \in G_{-} G_{+}$.

Pour $\omega \in \Omega_{g}, x$ écrivons

$g X_{n}^{-}(\omega)=d_{n}^{-}(g, \omega) d_{n}^{+}(g, \omega) \gamma_{n}(g, \omega) u_{n}(g, \omega)$ avec $d_{n}^{-} \in D_{-}, d_{n}^{+} \in D_{+}, \gamma_{n} \in \Gamma^{\prime}$ et $\tilde{u}_{n} \in \tilde{N}$;

$g Z(e, \omega)=d^{-}(g, \omega) d^{+}(g, \omega)(g, \omega) \tilde{u}(g, \omega)$ avec $d^{-} \in D_{-}, d^{+} \in D_{+}, \gamma \in \Gamma^{\prime}$ et $\tilde{u} \in \tilde{N}$.

Nous pouvons évidemment choisir les décompositions de $g X_{n}^{-}(\omega)$ et $g Z(e, \omega)$ de façon que les suites $d_{n}^{-}, d_{n}^{+}, \gamma_{n}$ et $\tilde{u}_{n}$ convergent respectivement vers $d^{-}, d^{+}, \gamma$ et $\tilde{u}$. Nous avons alors (en allégeant l'écriture)

$g x_{n}=d_{n}^{-} d_{n}^{+} \gamma_{n} \tilde{u}_{n} x_{n}^{+}$.

D'après la proposition (9.8.), $\boldsymbol{\mu}_{-}\left(\tilde{u}_{n} x_{n}^{+}\right)$converge vers e. Comme les suites $d_{n}^{+}$et $\gamma_{n}$ d'éléments respectivement de $D_{+}$et $\Gamma^{\prime}$ sont convergentes, on en déduit que $\mu_{-}\left(d_{n}^{+} \gamma_{n} \tilde{u}_{n} x_{n}^{+}\right)$converge vers $e$.

$\mu_{-}\left(g x_{n}\right)$ converge donc vers $d^{-}$et le théorème (9.7.) est démontré. 
10.1. Proposition. - Soit $G$ un groupe de Lie ayant un nombre fini de composantes connexes et $\mathrm{R}$ son radical. Soient $\mu$ une mesure de $M^{1}(G)$ étalée apériodique et $M=M_{-}^{\mu} M_{+}^{\mu}$ une décomposition du nilradical $M$ de $R$ en produit amalgamé de sous-groupes associée d̀ $\mu$ (voir (7.17.)). Alors les fonctions $\mu$-harmoniques bormées sur $\mathrm{M}_{\mu}{ }^{\mathrm{G}}$ sont les composées par l'application naturezle de $\mathrm{M}_{-}^{\mathrm{G}}$ sur $\mathrm{R}^{G}$ des fonctions $\xi(\mu)$-harmoniques bormées sur $R^{\mathrm{G}}$, où $\xi$ désigne $\eta^{\prime}$ 'application naturezle de $G$ sur $R^{G}$

Preuve. - On reprend les notations des paragraphes précédents qui ont été résumées en (9.0.). On omet l'indice " $\mu$ ".

Désignons par $\mu$ l'algèbre de Lie de $M$. Soit $\mathcal{L}$ la famille des sous-groupes fermés de $M$ contenant $M_{-}$. Soit $\mathbb{B}$ l'ensemble des éléments $X$ de $\mathcal{H}$ tels que les fonctions $\mu$-harmoniques bornées sur $X^{{ }^{G}}$ soient les composées par l'application naturelle de $x \backslash^{G}$ sur $R \backslash^{G}$ des fonctions $\mu$-harmoniques bornées sur $R^{G}$. Nous allons montrer que $\mathcal{H}=\mathcal{B}$. Donc en particulier $M_{-} \in \mathcal{S}_{3}$ et la proposition sera prouvée.

Montrons d'abord que $\mathcal{S}$ contient $M$. Soit $\left(\Omega, \mathscr{F}_{\mathcal{F}},\left(X_{n}\right)_{n} \in \mathbb{N},\left(\mathbb{P}_{g}\right)_{g} \in G\right)$ la marche aléatoire droite de loi $\mu$ sur $G$. Ecrivons (voir (9.5.)) $X_{n}=D_{n} K_{n}$ avec $D_{n} \in D$ et $k_{n} \in K$. Soit $p \in P$ et posons $U_{n}=D_{n} P D_{n}^{-1} P^{-1}$; comme $P$ commute avec $S, U_{n}$ est à valeurs dans $M$. Nous avons

$$
x_{n}^{-1} u_{n} p x_{n}=k_{n}^{-1} p k_{n} \subset \underset{k \in K}{U} k^{-1} p k \text {. }
$$

${ }^{A d} y^{K}$ étant compact, d'après le théorème (6.8.) il s'ensuit qu'il existe un voisinage de $e$ dans $P$ contenu dans le groupe des $\mu$-périodes (pour les détails voir la démonstration du lemme (7.9.)). P étant connexe, c'est $P$ tout entier qui est contenu dans ce groupe; par suite $\mathscr{S}_{3}$ contient $M$.

Supposons que $\boldsymbol{\beta}$ contiennent tous les éléments de $\mathcal{H}$ de dimension strictement supérieure à $\ell$, avec $\ell<\operatorname{dim} \mu$, et soit $x \in \mathcal{H}$ de dimension $\ell$. Désignons

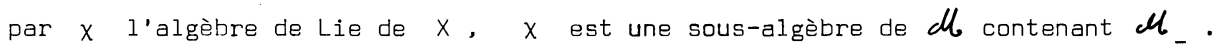
Puisque $\ell<\operatorname{dim} \mu, x$ ne peut contenir $\boldsymbol{H}$. Soit $\left(e_{i}\right)_{1} \leq i \leq m$ une base ordonnée de $\mu^{\mathbb{C}}$ qui triangularise simultanément les éléments de ad $\mathcal{M}^{\mathbb{C}} \mathfrak{D}$ et partant ceux de $A d \mu \mathbb{C}$. Notons $e_{i}$ le premier vecteur de cette base n'appartenant pas à $x^{\mathbb{C}}$ et $\xi^{\mathbb{C}}$ le sous-espace de dimension 1 de $\mu^{\mathbb{C}}$ engendré par le vecteur $e_{i_{0}}$. Posons

$$
\xi=\left\{t+\bar{t} \text { avec } t \in \xi^{\mathbb{C}}\right\} \text { et } x^{\prime}=x \boxplus \xi \text {. }
$$

Il est clair que l'image de $e_{i_{0}}$ dans $\mu / \mu_{\cap}$ est un vecteur propre pour la 
représentation adjointe de $D$ (resp. D) dans $\mu / \mu \cap x_{x}$. Par conséquent il existe $\lambda \in \Lambda \cap \Xi_{+}$(car $x$ contient $\mu_{-}$) tel que pour tout $Y$ de $D$, $\left[Y, e_{i_{0}}^{+}\right]=\lambda(Y) e_{i_{0}} \operatorname{modulo} \mu^{\mathbb{C}} \cap \chi^{\mathbb{C}}$.

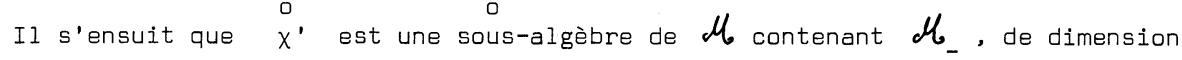
strictement supérieure à $l$; en outre nous avons

$[\xi, \xi] \subset x$ et donc $[\mu, \xi] \subset x$ (puisque $\mu_{0} \subset \xi$ ).

Reprenons les notations de (9.5.), nous avons $X_{n}=D_{n} K_{n}$ avec $D_{n} \in D$ et $K_{n} \in K$. Soit $x=t+\bar{t}$ avec $t \in \xi^{\mathbb{C}}$. Considérons la variable aléatoire,

$$
\begin{aligned}
& U_{n}=D_{n}\left[\exp \left(\phi_{\lambda}\left(D_{n}^{-1}\right) t+\phi_{\bar{\lambda}}\left(D_{n}^{-1}\right) \bar{t}\right)\right] D_{n}^{-1} \exp (-x) \\
& =\exp \left[\phi_{\lambda}\left(D_{n}^{-1}\right) \cdot A d D_{n}(t)+\phi_{\bar{\lambda}}\left(D_{n}^{-1}\right) \cdot A d D_{n}(\bar{t})\right] \exp (-x) \\
& =\exp \left[\phi_{\lambda}\left(D_{n}^{-1}\right) \cdot A d D_{n}(t)+\overline{\phi_{\lambda}\left(D_{n}^{-1}\right) \cdot A d D_{n}(t)}\right] \exp (-x)
\end{aligned}
$$

Comme $A d D_{n}(t)=\phi_{\lambda}\left(D_{n}\right) \cdot t+v_{n}(t)$ avec $v_{n}(t) \in \mu^{\mathbb{C}} \cap x^{\mathbb{C}}$, il s'ensuit que l'on a

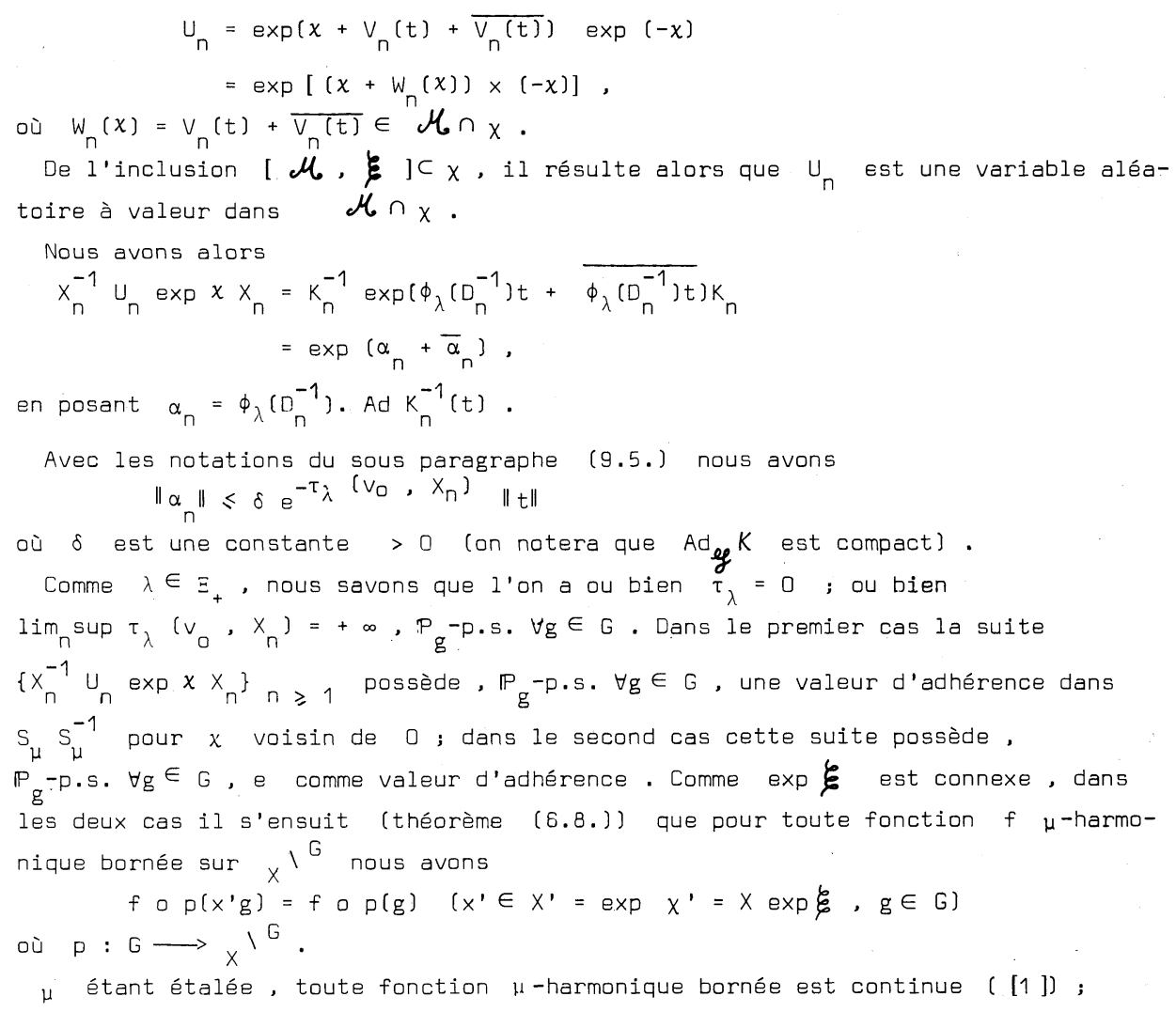


$f$ o $p$ est donc continue et on en déduit que

$$
f \circ p\left(x^{\prime} g\right)=f \circ p(g) \quad\left(x^{\prime} \in \vec{x}^{\prime}, g \in G\right) \text {, }
$$

ce qui montre que les fonctions $\mu$-harmoniques bornées sur $x \wedge^{G}$ sont les composées par l'application naturelle de $x \backslash^{G}$ sur $\overline{X^{\prime}} \backslash^{G}$ des fonctions $\mu$-harmoniques bornées sur $\bar{X}^{\backslash} \backslash^{G}$. L'hypothèse de récurrence permet alors de conclure que $x \in \mathcal{G}$. Nous venons donc de prouver par récurrence que $\mathcal{H} \subset \mathcal{B}$ et la proposition (10.1.) est démontrée.

10.2. Corollaire. - Soit $G$ un groupe de Lie ayant un nombre fini de composantes connexes. Soient $\mu$ une mesure de $M^{1}(G)$ étalée apériodique et $G=G_{-}^{\mu} E_{+}^{\mu}$ une décomposition de $G$ associée à $\mu$ (voir $\$ 7$ ). Alors les fonctions $\mu$-harmoniques bormées sur ${ }_{G}^{\mu} \backslash^{G}$ sont les composées par l'application naturelle de ${ }_{G_{-}^{\mu}}^{G}$ sur $i^{G}$ des fonctions $\mu$-harmoniques bormées sur ${ }_{D} \backslash^{G}$.

Preuve. - Nous avons $G_{-}^{\mu}=M_{-}^{\mu} N$. D'après la proposition (10.1.), les fonctions $\mu$-harmoniques bornées sur $\bar{\backslash}^{G}$ sont les composées par l'application naturelle de $G_{-}^{\mu} \backslash^{G}$ sur $R N \backslash^{G}$ des fonctions $\mu$-harmoniques bornées sur ${ }^{G N} \backslash^{G}$. Comme $[D, D] C R N$, le corollaire (10.2.) résulte alors du lemme (7.9.).

10.3.- Nous allons à présent étudier les fonctions $\mu$-harmoniques bornées sur ${ } \backslash^{G} \cdot \mu$ est une mesure de $M^{1}(G)$ étalée et apériodique.

Quitte à remplacer $G$ par $R \backslash^{G}$, on peut supposer que $G$ est un groupe de Lie ayant un nombre fini de composantes connexes dont la composante connexe $G_{0}$ de l'unité est semi-simple. Nous notons $G=N A K$ et $G=N A N \Gamma^{\prime}$ (resp. $G_{0}=N A K$ et $G_{0}=N A N \Gamma$ ) des décompositions d'Iwasawa et de Bruhat de $G$ (resp. de $G_{0}$ ). Si y est un élément de $G$, nous notons $\bar{y}$ sa composante sur $K$ pour la décomposition d'Iwasawa .

Dans le cas où $G_{0}$ est de centre fini, $K$ et $\Gamma^{\prime}$ sont des sous-groupes compacts de $G$, et la chaine de loi $\mu$ sur $K\left(\approx{ }_{N A}{ }^{G}\right)$ vérifie la condition de Doeblin (cor. (7.2.)). D'après [ 6$]$, nous savons alors que $K$ se décompose en un ensemble transient et une réunion finie de classes ergodiques; pour tout élément $g$ de $G, \overline{g X}_{n}$ finit par rentrer, $\mathbb{P}_{x}-$ p.s. $\forall x \in G$, dans une classe ergodique et y reste.

Dans le cas général (i.e. que $G_{0}$ peut avoir un centre infini), la chaine de loi $\mu$ sur $K$ ne vérifie plus la condition de Doeblin. Cependant, Rosenberg a prouvé ([ 24] ) que, pour la chaine de loi $\mu, K$ se décompose en un ensemble transient et en une réunion dénombrable de classes ergodiques; pour tout élément $g$ de $G, \overline{g X_{n}}$ finit par rentrer, $\mathbb{P}_{x}-$ p.s. $\forall x \in G$, dans une classe ergodique et 
y reste.

Plaçons nous dans le cas général et notons $E_{i}, i \in I$, les différentes classes ergodiques de la chaine de loi $\mu$ sur $K$, où I est un sous-ensemble, fini ou infini, de $\mathbb{N}$. Comme ncus avons $\overline{\gamma y}=\gamma \bar{y}\left(\gamma \in \Gamma^{\prime}, y \in G\right.$ ), (car $\Gamma^{\prime}$ normalise à la fois $N$, A et $K$ ), les éléments de $\Gamma^{\prime}$, permutent les classes ergodique ; c'est-à-dire que pour tout $i \in I$ et tout $Y \in \Gamma^{\prime}$, il existe $r(i, \gamma) \in I$ tel que

$$
\gamma E_{i}=E_{r(i, \gamma)} \cdot
$$

Notons aussi que puisque la chaine de loi $\mu$ sur $N$ A $\backslash^{G}$ est ergodique (lemme (7.7.)), pour tout $i$ et $j \in I$ il existe $\gamma_{i, j} \in \Gamma$ tel que $E_{j}=\gamma_{i}, j E_{i}(\operatorname{voir}[26], \$ 5)$.

Soit $E_{i_{0}}, i_{0} \in I$, une classe ergodique de la chaine de loi $\mu$ sur $K$ et choisissons un élément $x$ de $E_{i_{0}}$. Pour $\mathbb{P}_{x}$-presque tout $\omega, \overline{X_{n}(\omega)} \in E_{i_{0}}$ pour. tout $n \in \mathbb{N}$. En outre si $g \in G$, nous savons que pour $\mathbb{P}_{x}$-presque tout $\omega$, $\overline{g X_{n}(\omega)}$ finit par rentrer dans une classe ergodique $E_{i(g, \omega)}, i(g, \omega) \in I$, et $y$ reste.

Ecrivons $x_{n}=N_{n} A_{n} K_{n}$ avec $N_{n} \in N, A_{n} \in A$ et $K_{n} \in K$. Pour tout $g \in G$, la composante de $g X_{n}$ sur $N$ converge, $\mathbb{P}_{X}-p . s$. , vers une variable aléatoire $Z(g,$.$) . D'après (9.14$.$) , nous avons pour \mathbb{P}_{x}$-presque tout $\omega$

i) $\exists l(\omega) \in \mathbb{N}, \forall n \geqslant l(\omega), g N_{n}(\omega)=u_{n}(g, \omega) a_{n}(g, \omega) \gamma_{n}(g, \omega) \tilde{u}_{n}(g, \omega)$ avec $u_{n} \in N, a_{n} \in A, \gamma_{n} \in \Gamma^{\prime}$, et $\tilde{u}_{n} \in \tilde{N}$.

ii) Les suites $u_{n}, a_{n}, \gamma_{n}, u_{n}$ convergent respectivement vers $u, a, y$, $\tilde{u}$ tels que $g Z(e, \omega)^{n}=u(g, \omega)$ a $(g, \omega) \gamma(g, \omega) \tilde{u}(g, \omega)$.

On a alors, $\mathbb{P}_{x}$-presque sûrement, $\overline{g X_{n}}=\overline{\gamma_{n} A_{n}^{-1} \tilde{u}_{n} A_{n} \bar{X}_{n}}$, avec $\gamma_{n}(g,.) \longrightarrow \gamma(g,$.$) et A_{n}^{-1} \tilde{u}_{n} A_{n} \longrightarrow$ e, (llemme (9.9.)).

$\mu$ étant étalée, chaque classe ergodique de la chaine de loi $\mu$ sur $K\left(\tilde{\sim}_{N A} \backslash G\right)$ contient un ouvert de $K$. En effet si $E$ est une classe ergodique, il existe une unique mesure de probabilité $\mu$-invariante $\Theta$ portée par $E$. D'après [1] on sait que $\Theta$ est la restriction à un ouvert de. $K$ d'une mesure quasi-invariante sur $K$. E contient donc un ouvert de $K$. De ce qui précède, il résulte alors que l'on a :

$$
\left.E_{i(g, .)}=\gamma(g, .) E_{i_{0}}=E_{r\left(i_{0}\right.}, \gamma(g, .)\right), \mathbb{P}_{x}-p . s . .
$$

Soit $f$ une fonction $\mu$-harmonique bornée sur $K\left(\tilde{\sim}_{N A} \backslash{ }^{G}\right)$. Pour $i \in I$, la restriction, $f_{E_{i}}$, de $f$ à $E_{i}$ est une fonction harmonique bornée pour la chaine de loi $\mu$ restreinte à $E_{i}$. Or cette chaine est une chaine récurrente au sens de Harris (voir [6] et [23]) pour la mesure de Haar sur $K$, on 'sait alors 
(voir [23]) que $f_{E_{i}}$ est $m_{K}-p . s$. constante. Mais $\mu$ étant étalée, $f$ et par sujte $f_{E_{i}}, i \in I$, sont continues $([1])$; il s'ensuit donc que $f$ est constante sur chaque classe ergodique. Posons $f_{E_{i}}=a_{i} \in \mathbb{R}$; pour $\mathbb{P}_{x}$-presque tout $\omega$ de $\Omega$, il existe $\ell(\omega) \in \mathbb{N}$ tel que pour tout entier $n \geqslant \ell(\omega)$

$$
f\left(\overline{\left.g X_{n}(\omega)\right)}=f(\gamma(g, \omega) \times)=a_{r\left(i_{0}\right.}, \gamma(g, \omega)\right) \text {. }
$$

Soit $\Gamma_{\mu}^{\prime}=\left\{\gamma \in \Gamma^{\prime}: r\left(i_{0}, \gamma\right)=i_{0}\right\} ; \Gamma_{\mu}^{\prime}$ est un sous-groupe distingué de $\Gamma^{\prime}$ contenant $\Gamma_{0}^{\prime}$. Désignons par $t$ l'application naturelle de $\Gamma^{\prime}$ sur $\Gamma^{\prime} \Gamma_{\mu}^{\prime}$ et posons

$$
\hat{f}(t(\gamma))=f(\gamma x) \quad\left(\gamma \in \Gamma^{\prime}, x \in E_{i_{0}}\right) .
$$

Alors $\hat{f}$ est une fonction continue sur $\Gamma^{\prime} / \Gamma_{\mu}^{i_{0}}$ et nous avons

i) $\left.f(\bar{g})=\mathbb{E}_{x}\left[\lim \overline{f\left(g x^{-1} x_{n}\right.}\right)\right]=\mathbb{E}_{x}\left[\hat{f} \operatorname{fot}\left(\gamma\left(g x^{-1},.\right)\right]\right] \quad(g \in G)$

ii) Soit $g \in G$, pour $\mathbb{P}_{x}$-presque tout $\omega$,

$\exists l(\omega) \in \mathbb{N}, \forall n \geqslant l(\omega) \quad \overline{f\left(g X_{n}(\omega)\right)}=\hat{f o t}(\gamma(g, \omega))$.

Pour $i \in I$, notons $\tau_{i}$ l'élément de $\Gamma^{\prime} /_{\Gamma_{\mu}^{\prime}}^{\prime}$ dont un quelconque représentant $\gamma_{i}$ dans $\Gamma^{\prime}$ vérifie $\gamma_{i} E_{i_{0}}=E_{i}$. Nous avons alors

$$
f(\bar{g})=\sum_{i \in I} a_{i} \mathbb{P}_{x}\left[t\left(\gamma\left(g x^{-1}, .\right)\right)=\tau_{i}\right] \text {. }
$$

10.4. Démonstration du théorème (8.4.). - Soit $G$ un groupe de Lie ayant un nombre fini de composantes connexes. Soient $\mu$ une mesure de $M^{1}(G)$ étalée, apériodique, possèdant un moment d'ordre 1 et $G=G_{-}^{\mu} E_{+}^{\mu}$ une décomposition de $G$ associée à $\mu$. Dans la suite nous omettons l'indice " $\mu$. .

Nous identifions $E_{+}$et $G_{-}{ }^{G}$; nous désignons par q l'application naturelle de $G$ sur $G_{-} l^{G}$ et par $n^{G_{-}} l^{\prime}$ homéomorphisme de $G_{-} \times G_{-} \backslash^{G}$ sur $G$. Posons

$$
g \cdot(u, v)=n^{-1}(g \cdot n(u, v))\left(g \in G,(u, v) \in G_{-} \times G_{-} l^{G}\right) \text {; }
$$

$G_{-} \times G_{-} \backslash^{G}$ est alors un G-espace, G-homéomorphe à $G$.

Soit $f$ une fonction $\mu$-harmonique bornée $\mathrm{u.c.g.} \mathrm{,} \mathrm{nous} \mathrm{savons} \mathrm{que} \mathrm{pour} \mathrm{tout} g$ de $G, \lim f\left(g x_{n}\right)$ existe, $\mathbb{P}_{x}-p . s . \forall x \in G$. D'après le théorème (9.2.), la composante de $g_{0} X_{n}$ sur $G_{-}$converge, $\mathbb{P}_{x}-p . s . \forall x \in G$, vers une variable aléatoire $z(g,$.$) . Posons x_{n}=x_{n}^{-} x_{n}^{+}$avec $x_{n}^{\underline{x}} \in G_{-}$et $x_{n}^{+} \in E_{+}$. Pour tout $x$ et $g \in G$, il existe un sous-ensemble mesurable $\Omega_{\mathrm{g}}, x$ de $\Omega$ de $\mathbb{P}_{x}$-mesure 1 tel que :

i) $\forall_{\omega} \in \Omega_{g}, x, \lim _{n} x_{n}^{-}(\omega)$ existe et est égal à $z(e, \omega)$

ii) $\forall \omega \in \Omega_{g}, x$, les limites $\lim _{n} f\left(g x_{n}(\omega)\right)$ et $\lim f\left(g z(e, \omega) x_{n}^{+}(\omega)\right)$ existent et sont égales pour tout $g \in G$ (on notera que $f$ est u.c.g.). 
$G$ étant séparable, soit $\left(g_{i}\right)_{i} \in \mathbb{N}$ une suite dense dans $G$. Posons pour $x \in G$,

$f$ étant u.c.g. nous avons alors

$$
\Omega_{x}=\hat{n}_{i \in \mathbb{N}}{ }^{\Omega} g_{i}, x
$$

i) $\mathbb{P}_{x}\left[\Omega_{x}\right]=1$

ii) $\forall \omega \in \Omega_{x}, \lim _{n} x_{n}^{-}(\omega)$ existe et est égal à $z(e, \omega)$

iii) $\forall \omega \in \Omega_{x}$, les $\operatorname{limites} \lim _{n} f\left(g x_{n}(\omega)\right)$ et $\underset{n}{\lim } f\left(g Z(e, \omega) x_{n}^{+}(\omega)\right)$ existent et sont égales pour tout $g$ de $G$.

En particulier, pour $\omega$ donné on peut choisir $g=g^{\prime} z(e, \omega)^{-1}$ où $g^{\prime}$ est un élément quelconque de $G$; on voit alors que $\underset{n}{\lim } f\left(g^{\prime} x_{n}^{+}(\omega)\right)$ existe pour tout $\omega \in \Omega_{x}$ et tout $g^{\prime} \in G$.

Soit $f$ la fonction $f \circ n^{-1}$, nous avons alors

i) $\tilde{f} \in C_{U}\left(G_{-} \times G_{-} \backslash^{G}\right)$

ii) $\forall \omega \in \Omega_{x}$ les limites $\lim _{n} \tilde{f}\left(v, q\left(g x_{n}(\omega)\right)\right)$ et $\lim _{n} \tilde{f}\left(v, q\left(g Z(e, \omega) x_{n}^{+}(\omega)\right)\right)$ existent et sont égales pour tout $(V, g) \in G_{-} \times G$.

Considérons la fonction $\phi$ définie sur $G_{-} \times G_{-} \backslash^{G}$ par

$$
\phi(v, q(g))=\mathbb{E}_{g}\left[\lim _{n} \tilde{f}\left(v, . q\left(x_{n}\right)\right)\right] \quad\left(v \in G_{-}, g \in G\right) .
$$

Pour tout $\vee \in G_{-}, \phi(v,$.$) est une fonction \mu$-harmonique bornée sur $G_{-} G$. Désignons par $\xi$ l'application naturelle de $G$ sur $R{ }^{G}$. D'après le corolilaire (10.2.) il existe une fonction $\bar{\phi}$ définie sur $G_{-} \times \xi_{\xi(D)} \backslash \xi(G)$ (noter que $\xi(D) \backslash^{\xi(G)}$ et $D \backslash^{G}$ sont homéomorphes] telle que

$$
\bar{\phi}(v, p o \xi(g))=\phi(v, q(g)) \quad\left(v \in G_{-}, g \in G\right),
$$

où $P$ désigne l'application naturelle de $\xi(G)$ sur $\xi(D) \backslash \xi(G)$.

Soit $v \in G_{-}$et $g \in G$ fixés. D'après la proposition (6.7.) nous avons

$$
\lim _{n} \bar{\phi}\left(v, \text { po } \xi\left(g x_{n}\right)\right)=\lim _{n} \underset{\sim}{f}\left(v, q\left(g x_{n}\right)\right), \mathbb{P}_{x}-p \cdot s . \forall x \in G \text {. }
$$

Désignons par $I_{y}$ l'automorphisme intérieur de $G, g \longmapsto y^{-1} g y$. Il est alors facile de voir que si le théorème (8.4.) est vérifié pour un élément de $\left\{I_{y}(\mu), y \in G\right\}$ il l'est pour tous. Il suffit en effet de remarquer que $f$ est $\mu$-harmonique bornée sur $G$ si et seulement si $f \circ I_{y}^{-1}$ est $I_{y}(\mu)$-harmonique bornée sur $G$. Reprenons pour $\xi(G)$ les notations de (10.3.), quitte à remplacer $\mu$ par $I_{y}(\mu), y \in G$, nous pouvons supposer que $\xi(e) \in E_{i_{0}}$. D'après (10.3.) nous savons alors que pour $\mathbb{P}_{e}$-presque tout $\omega$ de $\Omega, i$ existe $\ell(\omega) \in \mathbb{N}$ tel que pour tout $n \geqslant \ell(\omega)$,

$$
\left.\bar{\phi}\left(v, \operatorname{pog}\left(g x_{n}\right)(\omega)\right)\right)=\hat{f}(v, t(\gamma(g, \omega))) \text {, }
$$

où $\hat{f}$ est une fonction définie sur $G_{-} \times \Gamma^{\prime} /_{\Gamma_{\mu}^{\prime}}$. 
Nous avons pour tout couple $(v, g)$ de $G_{-} \times G$,

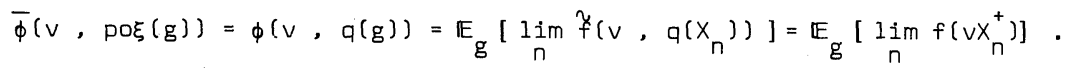

D'où

$(v, g) \in \sup _{-} \times G|\bar{\phi}(h v, \operatorname{pog}(g))-\bar{\phi}(v, \operatorname{pog}(g))| \leqslant \sup _{g \in G}\left|f^{h}(g)-f(g)\right|=\left\|f^{h}-f\right\|_{\infty}$. Comme $f$ est U.c.g. , il s'ensuit que pour tout $\bar{\gamma} \in \Gamma^{\prime} /_{\Gamma_{\mu}^{\prime}}$ et tout $g \in G$ les
fonctions $v \longmapsto \hat{f}(v, \bar{\gamma})$ et $v \longmapsto \bar{\phi}(v, \operatorname{pog}(g))\left(v \in G_{-}\right)$, sont u.c.g. $\tilde{f}$ appartenant à $C_{U}\left(G_{-} \times{ }_{G_{-}}{ }^{G}\right)$ et $G$ étant séparable, il résulte alors qu'il existe, pour tout $g \in G$, uñ sous-ensemble mesurable $\Omega_{g} \subset \Omega_{e}$ de

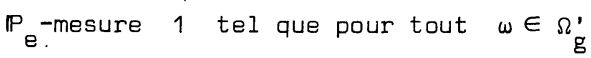
$\lim _{n} \bar{\phi}\left(v, \operatorname{pog}\left(g x_{n}(\omega)\right)\right)=\lim _{n} \tilde{f}\left(v, q\left(g x_{n}(\omega)\right)\right)=\hat{f}(v, t(\gamma(g, \omega))), \forall v \in G_{-}$. En outre comme $\Gamma^{\prime} / \Gamma_{\mu}^{\prime}$ est discret, la fonction $\hat{f}$ est continue sur $G_{-} \times \Gamma^{\prime} /_{\Gamma_{\mu}}$. Soit $g \in G$ et $\omega^{\mu} \in \Omega_{g}^{\prime}$ nous avons, en posant

$$
\begin{aligned}
g x_{n}(\omega)=\left(g x_{n}(\omega)\right)^{-}\left(g X_{n}(\omega)\right)^{+} & \operatorname{avec}\left(g x_{n}(\omega)\right)^{-} \in G_{-} \text {et }\left(g x_{n}(\omega)\right)^{+} \in E_{+}, \\
\lim _{n} f\left(g X_{n}(\omega)\right) & \left.=\lim _{n} f\left(g x_{n}(\omega)\right)^{-}\left(g x_{n}(\omega)\right)^{+}\right) \\
& =\lim _{n} f\left(z(g, \omega)\left(g x_{n}(\omega)\right)^{+}\right) \\
& =\lim _{n} \tilde{f}\left(z(g, \omega), q\left(g x_{n}(\omega)\right)\right) \\
& =\lim _{n} \bar{\phi}\left(z(g, \omega), \operatorname{pog}\left(g x_{n}(\omega)\right)\right) \\
& =\hat{f}(Z(g, \omega), t(\gamma(g, \omega))) .
\end{aligned}
$$

Considérons la décomposition $G=G_{-} G_{+}$, modulo $m_{G}$, associée à $\mu$ (voir (7.17.)); nous avons avec les notations des paragraphes précédents

$$
G_{+}=M_{+} P A \tilde{N} \Gamma^{\prime} \text { (et } E_{+}=M_{+} P A K \Gamma^{\prime}=M_{+} P A K \text { ) }
$$

Posons $H_{+}=M_{+} P A \tilde{N} \Gamma_{\mu}^{\prime} ; G \times \Gamma^{\prime} / \Gamma_{\mu}^{\prime}$ est homéomorphe à un ouvert de $G /_{H_{+}}$. Soit $U(g, \omega)$ (resp. $U(e, \omega))$ l'élément de $G / H_{+}$correspondant à $(Z(g, \omega)$, $t(\gamma(g, \omega)))$ (resp. à $(Z(e, \omega), t(e)))$. D'après le théorème (9.7.), nous avons

$$
U(g, .)=g \cdot U(e, .) \mathbb{P}_{e}^{-p . s . ;}
$$

et par suite, en restreignant au besoin $\Omega^{\prime} \mathrm{g}$, nous avons

(*) $\lim f\left(g x_{n}(\omega)\right)=\bar{f}(g \cdot U(e, \omega)) \quad \forall \omega \in \Omega_{g}^{\prime}$ où $\bar{f}$ est une fonction continue (car $\hat{f} l$ l'est) définie sur l'ouvert $X$ de $G / H_{+}$image de $G_{-} \Gamma^{\cdot}$ par l'application naturelle de $G$ sur $G_{H_{+}}$. Montrons que pour tout $\varepsilon>0$, il existe un voisinage $V_{\varepsilon}$ de $e$ dans $G$ tel que pour tout $g$ de $V_{\varepsilon}$ et tout $w$ de $X$ avec $g \cdot w \in X$ on ait

$$
|\bar{f}(g \cdot w)-\bar{f}(w)|<\varepsilon \text {. }
$$


$f$ étant u.c.g. , pour tout $\varepsilon>0$, il existe un voisinage $V_{\varepsilon}$ de $e$ dans $G$ tel que pour tout $g \in V_{\varepsilon}$ on ait $\left\|f^{g}-f\right\|_{\infty}<\varepsilon$. Soit $g \in V_{\varepsilon}$ et $y \in G$. nous avons pour tout $\omega \in \Omega_{y}^{\prime} \cap \Omega_{g y}^{\prime}$

$|\bar{f}(g y \cdot u(e, \omega))-\bar{f}(y \cdot u(e, \omega))|=\lim _{n}\left|f\left(g y x_{n}(\omega)\right)-f\left(y x_{n}(\omega)\right)\right| \leqslant\left\|f^{g}-f\right\|_{\infty}<\varepsilon$. D'où, pour tout $g \in V_{\varepsilon}$ et tout $y \in G$, nous avons

$|\bar{f}(g \cdot(y \cdot w))-\bar{f}(y \cdot w)|<\varepsilon$

pour $v$-presque tout $w$, où $v$ désigne la loi de variable aléatoire U(e, .) définie sur $\left(\Omega, \mathscr{F}, \mathbb{P}_{e}\right)$.

Or $\nu$ est une mesure $\mu$-invariante sur $G / H_{+}$; d'après [1] , on sait que $\nu$ est la restriction à un ouvert de $G / H_{+}$(contenu dans $X$ ) d'une mesure quasi-invariante. $\bar{f}$ étant continue sur $X$, il s'ensuit que pour tout $g$ de $V_{\varepsilon}$ on a $|\bar{f}(g \cdot w)-\bar{f}(w)|<\varepsilon$ pour tout $w \in X$ tel que $g \cdot w \in X$.

Soit $w \in G / H_{+} ; w$ est l'image dans $G / H_{+}$d'un élément $x$ de $G$. Comme $\left\{g \in G: g \times \notin G_{-}^{+} G_{+}\right\}$est de mesure de Haar nulie, on peut trouver une suite $\left(g_{n}\right)_{n} \geqslant 0$ d'éléments de $G$ convergeant vers e telle que $g_{n} \times \in G_{-} G_{+}$, pour tout entier naturel $n$. Nous avons alors $g_{n} \cdot w \in X$, pour tout $n \in \mathbb{N}$, avec $g_{n} \longrightarrow e$. D'après ce qui précède il s'ensuit que la suite réelle $\left(\bar{f}\left(g_{n} \cdot w\right)\right)_{n} \geqslant 0$ est de Cauchy; posons $\bar{f}(w)=\lim \bar{f}\left(g_{n} \cdot w\right)$. Il est facile de voir que cette limite est indépendante du choix de $l a^{n}$ suite $\left(g_{n}\right)_{n} \in \mathbb{N}$ et que l'on définit ainsi une fonction de $C_{u}\left(G / H_{+}\right)$qui prolonge $\bar{f}$; nous notons encore $\bar{f}$ cette fonction.

De la relation [*] il s'ensuit que

$f(g)=\mathbb{E}_{g}\left[\lim f\left(x_{n}\right)\right]=\mathbb{E}_{e}\left[\lim f\left(g x_{n}\right)\right]=\mathbb{E}_{e}[\bar{f}(g \cdot U(e,))]$,

soit en notant $\nu$ la loi de la variable aléatoire $U(e$, , ) définie sur $\left(\Omega, \mathcal{F}, \mathbb{P}_{e}\right)$

$$
\text { (**) } f(g)=\int G_{H_{+}} \bar{f}(g \cdot w) v(d w) \text {. }
$$

De $[*]$ et $(* *)$ il résulte que l'on a $\|f\|_{\infty}=\|\bar{f}\|_{\infty}$. On en déduit donc que l'application $\bar{f} \longmapsto \bar{f} * \nu$ de $\mathrm{C}_{u}\left(\mathrm{G} / \mathrm{H}_{+}\right)$sur $H_{\mu}$ est une G-isométrie. Le fait que l'application $\bar{f} \longmapsto \bar{f} * v$, où $\bar{f} \in L^{\infty}\left(G / H_{+}\right)$soit une G-isométrie de $L^{\infty}\left(G / H_{+}\right)$sur $E_{\mu}$ se montre alors en reprenant la démonstration du théorème I.3. de [1] . Le théorème (8.4.] est démontré dans le cas où $\mu$ est apériodique. Le cas général se déduit aisément de ce cas particulier, en reprenant la démonstration de la proposition IV.1. de [1]. 
11.1. Définition. - Soit G un groupe L.C.D. et M un G-espace localement compact; nous appelons G-compactification de $M$, tout couple $(\tilde{M}, \varepsilon)$ formé d'un G-espace compact $\tilde{M}$ et d'une application $\varepsilon$ de $M$ dans $\tilde{M}$ qui est un G-homéomorphisme de $M$ sur un sous-espace G-invariant et partout dense de $\tilde{M}$. Par abus de langage, nous disons aussi que $\tilde{M}$ est une G-compactification de $M$, en sousentendant l'application $\varepsilon$.

11.2. Familles discriminantes restreintes d'A-cocycles associées à $G$. - Soient $G$ un groupe de Lie ayant un nombre fini de composantes connexes et $\left\{\rho_{\lambda}, \lambda \in \Xi\right\}$ la famille discriminante d'A-cocycles obtenue pour la décomposition $G=D K$ de $G$ (voir $\$ 2)$. E est la réunion des ensembles de poids, $\Lambda$ et $\Sigma_{1}$, de la représentation adjointe de $\mathbb{D}$ respectivement dans $\mu^{\mathbb{C}}$ et $\xi^{\mathbb{C}} / \mu^{\mathbb{C}}$ (voir (5.5)). Nous savons que la famille $\left\{\rho_{\lambda}, \lambda \in \Sigma_{1}\right\}$ est composée d'éléments strictement négatifs pour toute mesure de $M^{1}(G)$ étalée (corollaire (7.15)).

Soit $\Lambda_{0}$ l'ensemble des poids de la représentation adjointe de $\mathbb{D}$ dans $\mu \mathscr{C} /[\mu, \mu]^{\mathbb{C}}$. Nous savons que tout élément de $\Lambda$ est une somme d'éléments de $\Lambda_{0}$ et par suite tout élément de $\left\{\rho_{\lambda}, \lambda \in \Lambda\right\}$ est une somme d'éléments de $\left\{\rho_{\lambda}, \lambda \in \Lambda_{0}\right\}$. Nous disons que $\left\{\rho_{\lambda}, \lambda \in \Lambda_{0}\right\}$ est une famille discriminante restreinte d'A-cocycles associée à $G$. Il est facile de voir (cf. (2.12)) que toute autre famille discriminante restreinte d'A-cocycles s'écrit $\left\{\rho_{\lambda} \circ\left(J_{d}, I_{d}\right), \lambda \in \Lambda_{o}\right\}$, où $d$ est un élément de $D, I_{d} I^{\prime}$ automorphisme intérieur de $G$, $g \longmapsto d^{-1}$ gd et $J_{d} l$ 'homéomorphisme de $B(G)$ sur lui-même qui à $u \in B(G)$ associe ن..d.

11.3. Théorème. - Soit $G$ un groupe de Lie ayant un nombre fini de composantes connexes. Soit $\mu \in M^{1}(G)$ étalée; désignons par $\pi_{\mu}$ (resp. $\Pi_{\xi(\mu)}$ ) l'espace de Poisson de $\mu$ (resp. $\xi(\mu)$ ) sur $G$ (resp. $\mathcal{R}^{G}$ ). Soit $\left\{\rho_{\lambda}, \lambda \in \Lambda_{0}\right\}$ une famizle discriminante restreinte $d^{\prime}$ A-cocycles associée au sous-groupe fermé $G_{\mu}$ engendré par le support de $\mu$ (voir (11.2)). Alors si pour tout $\lambda \in \Lambda_{0}$, $\rho_{\lambda}$ est $\mu$-positif (voir (7.11)), les G-espaces $\Pi_{\mu}$ et $\Pi_{\xi(\mu)}$ sont G-homéomorphes.

Supposons de plus que $\mu$ possède un moment d'ordre 1. Alors l'espace de Poisson $\Pi_{\mu}$ de $\mu$ est homogène, (et coincide avec $\Lambda_{\mu}$ ), si et seulement si les deux conditions suivantes sont satisfaites :

i) Les $\rho_{\lambda}, \lambda \in \Lambda_{0}$, sont tous $\mu$-positifs.

ii) $Z\left({ }_{R} \backslash^{G}\right) \cap T_{\xi(\mu)} T_{\xi(\mu)}^{-1}$ est d'indice fini dans le centre $Z\left({ }_{R}{ }^{G}\right)$ de ${ }_{R}{ }^{G}$, où 
$T_{\xi(\mu)}$ désigne le semi-groupe fermé de $R^{{ }^{G}}$ engendré par $\xi(\mu)$. Lorsque l'une de ces conditions n'est pas vérifiée, $\Pi_{\mu}$ est une G-compactification (définition (11.1)), non métrisable, de $\Lambda_{\mu}$

La preuve du théorème (11.3) résultera des trois lemmes suivants.

11.4. Lemme. - Soit M un G-espace homogène Zocalement compact. Alors il existe une G-compactification $(\tilde{M}, \varepsilon)$ de $M$, unique à un G-isomorphisme près, telle que Z'application $f \longmapsto f \circ \varepsilon$ soit une G-isométrie de $C(\tilde{M})$ sur $C_{U}(M)$. Les sousespaces $\varepsilon(M)$ et $\tilde{M}-\varepsilon(M)$ de $\tilde{M}$ sont G-invariants et $\tilde{M}$ n'est homogène que si $M$ est compact.

Preuve. - Nous donnons une démonstration brève de ce lemme qui est à rapprocher de la compactification de Cech d'un espace localement compact ([17]). Soit

$$
C_{U}^{1}(M)=\left\{f \in C_{U}(M) ; 0 \leqslant f \leqslant 1\right\} ;
$$

considérons $F=[0,1]^{C_{u}^{1}(M)}, 1$ 'application

$$
\begin{aligned}
\varepsilon: & M \longrightarrow F \\
& x \longrightarrow(f(x)) \quad f \in C_{U}^{1}(M)
\end{aligned}
$$

et I'adhérence $\tilde{M}$ de $\varepsilon(M)$ dans $F$. G opère sur $F$ de la façon suivante

$$
g \in G, \quad y=\left(y_{f}\right)_{f \in C_{u}^{1}(M)} \in F, \quad g \cdot y=\left(y_{f}\right)_{f \in C_{u}^{1}(M)} .
$$

On montre que cette action de $G$ induit sur $\tilde{M}$ une structure de G-espace et que le couple $(\tilde{M}, \varepsilon)$ constitue une G-compactification de $M$ ayant la propriété vou1ue.

L'unicité de $\tilde{M}$ à un G-isomorphisme près découle du corollaire de la proposition I.1 de [1].

Enfin $\varepsilon(M)$ est G-invariant; si $g \in G_{\sim}$ et $L_{g}$ désigne l'homéomorphisme $\tilde{M} \rightarrow \tilde{M}, L_{g}$ laisse $\varepsilon(M)$ et par suite $\tilde{M}-\varepsilon(M)$ invariants.

$x \longmapsto g \cdot x$

11.5. Lemme. - Soit G un groupe L.C.D. et $\mu \in M^{1}(G)$. Soient $(M, v)$ et $\left(M^{\prime}, v^{\prime}\right)$ deux $\mu$-espaces homogènes de $G$ tels que l'application $f \longmapsto f * v$ (resp. $\left.f \longmapsto f * v^{\prime}\right)$ de $C_{u}(M)$ (resp. $C_{u}\left(M^{\prime}\right)$ ) dans $H_{\mu}$ soit une G-isométrie. Alors les u-espaces homogènes $(M, v)$ et $\left(M^{\prime}, v^{\prime}\right)$ sont G-homéomorphes. 
Preuve. - Soit $(\tilde{M}, \varepsilon)$ (resp. $\left.\left(\tilde{M}^{\prime}, \varepsilon^{\prime}\right)\right)$ une G-compactification de $M$ (resp. $\overline{\left.M^{\prime}\right) . \tilde{M}}$ et $\tilde{M^{\prime}}$ (resp. $\varepsilon(\nu)$ et $\varepsilon^{\prime}\left(\nu^{\prime}\right)$ ) sont alors deux versions de l'espace de Poisson (resp. du noyau de Poisson) de $\mu$. D'après [1], il existe donc un G-homéomorphisme $\tilde{\phi}$ de $\tilde{M}$ sur $\tilde{M}$ ' tel que $\tilde{\phi}(\varepsilon(\nu))=\varepsilon^{\prime}\left(\nu^{\prime}\right)$. Il s'ensuit que $\left\{x \in M^{\prime}: \tilde{\phi}(\varepsilon(x)) \in \varepsilon^{\prime}\left(M^{\prime}\right)\right\}$ est non vide. Par suite $M$ et $M^{\prime}$ étant homogène, nous avons $\tilde{\phi}(\varepsilon(M))=\varepsilon^{\prime}\left(M^{\prime}\right)$ et l'application $\phi=\varepsilon^{\prime-1} \circ \tilde{\phi}$ o $\varepsilon$ est un G-homéomorphisme de $M$ sur $M^{\prime}$ envoyant $\nu$ sur $\nu^{\prime}$.

11.6. Lemme. - Soit G un groupe L.C.D. compactement engendré et M un G-espace homogène, alors $\dot{C}_{U}(M)$ est séparable si et seulement si $M$ est compact. D'autre part, pour toute $G$-compactification $(\tilde{M}, \varepsilon)$ de $M$, l'espace $\varepsilon(M)$ est séquentiellement fermé dans $\tilde{M}$.

Preuve. - Soit $x_{0}$ un point de $M$ et $H$ le sous-groupe de stabilité de $x_{0}$; $M$ est alors $G$-homéomorphe à $G / H$. Si $g \in G$ nous notons $\bar{g}$ l'image de $g$ par l'application naturelle de $G$ sur $G / H_{H}$.

Soit d une distance principale sur G (voir (6.3)) ; on définit

$$
\delta(\bar{g})=\inf _{h \in H} d(e, g h) \quad(g \in G) .
$$

$\delta$ est une application continue de $G /_{H}$ dans $\mathbb{R}_{+}$qui est bornée si et seulement si $G / H$ est compact; en effet si $\delta$ est majorée strictement par un entier $k$, du fait que $d$ est principale, il s'ensuit que $G$ est contenu dans $V^{k} H$ où $V$ est un voisinage compact de $e$ engendrant $G$.

D'autre part pour $(g, u) \in G \times G$, nous avons

et

$$
\delta(\overline{g u})=\inf _{h \in H} d(e, g u h) \leqslant \inf _{h \in H}(d(e, g)+d(e, u h))=d(e, g)+\delta(\bar{u})
$$

$$
\delta(\bar{u})=\inf _{h \in H} d\left(e,\left(g^{-1} g\right) u h\right) \leqslant \inf _{h \in H}\left(d\left(e, g^{-1}\right)+d(e, g u h)\right)=d(e, g)+\delta(\overline{g u}) .
$$

Autrement dit, nous avons

$$
|\delta(g \cdot \bar{u})-\delta(\bar{u})| \leqslant d(e, g) .
$$

Si $M$ est compact, $C_{u}(M)$ est séparable; supposons donc que $M$ n'est pas compact. Si $C_{u}(M)$ était séparable, pour toute suite $\left(x_{n}\right)_{n \in \mathbb{N}}$ de $M\left(\tilde{\sim} G /{ }_{H}\right)$ on pourrait extraire (par le procédé diagonal) une sous-suite $\left(x_{n_{k}}\right)_{k \geqslant 1}$ telle que la suite $\left(f\left(x_{n_{k}}\right)\right)_{k \geqslant 1}$ convergerait pour toute fonction $f$ de $C_{u}(M)$. Mais nous allons construire, pour toute suite $\left(x_{n}\right)_{n \geqslant 1}$ de $M$ telle que $\underset{n}{\lim .} \delta\left(x_{n}\right)=+\infty$, une fonction $\psi$ de $C_{u}(M)$ telle que la suite $\left(\psi\left(x_{n}\right)\right)_{n \geqslant 1}$ ne converge pas. 
Soit $U$ un voisinage compact symétrique de $e$ dans $G$ et $\left(x_{n}\right)_{n \geqslant 1}$ une suite d'éléments de $G / H$ telle que $\delta\left(x_{n}\right) \rightarrow+\infty$. Quitte à remplacer la suite donnée par une sous-suite, nous pouvons supposer que

$$
\delta\left(x_{n+1}\right) \geqslant \delta\left(x_{n}\right)+\sup _{g \in U} d(e, g) .
$$

Posons $B_{n}=\left\{x \in G / H: \delta(x) \leqslant \delta\left(x_{n}\right)\right\}$. Il est facile de voir, utilisant les relations (1) et (2) ci-dessus, que l'on a :

$$
\text { U. }\left(B_{n+1}-B_{n}\right) \subset B_{n+2}-B_{n-1} \text {. }
$$

Définissons alors la fonction :

$$
\psi(x)= \begin{cases}\frac{\delta\left(x_{2 n+1}\right)-\delta(x)}{\delta\left(x_{2 n+1}\right)-\delta\left(x_{2 n}\right)} & \text { si } x \in B_{2 n+1}-B_{2 n}, \quad n \geqslant 1 \\ \frac{\delta(x)-\delta\left(x_{2 n-1}\right)}{\delta\left(x_{2 n}\right)-\delta\left(x_{2 n-1}\right)} & \text { si } x \in B_{2 n}-B_{2 n-1}, n \geqslant 1 \\ 0 & \text { si } x \in B_{1} .\end{cases}
$$

Nous avons, pour tout $n \geqslant 1, \psi\left(x_{2 n-1}^{i}\right)=0$ et $\psi\left(x_{2 n}\right)=1$, donc $\left(\psi\left(x_{n}\right)\right)_{n} \in \mathbb{N}$ ne converge pas. Nous laissons au lecteur le soin de se convaincre, en utilisant les relations (1), (2) et (3), que $\psi$. appartient à $C_{U}(G / H)$.

D'autre part soient $(\tilde{M}, \varepsilon)$ la G-compactification de $M\left(\approx G /{ }_{H}\right)$ du lemme (11.4) et $\left(\varepsilon\left(x_{n}\right)\right)_{n \geqslant 1}$ une suite d'éléments de $\varepsilon(M)$ convergeant vers un élément $x=\left(x_{f}\right)_{f \in C_{u}^{1}(M)}$ de $\tilde{M}$. D'après ce qui précède la suite $\left(\delta\left(x_{n}\right)\right\}_{n \geqslant 1}$ ne peut tendre vers l'infini. Il s'ensuit donc que la suite $\left(x_{n}\right)_{n \geqslant 1}$ admet une valeur d'adhérence $y$ dans $M$ et par suite nous avons nécessairement $\varepsilon(y)=x$. $\varepsilon(M)$ est donc séquentiellement fermé dans $\tilde{M}$.

11.7. Démonstration du théorème $(11.3)$. - D'après $((8.5), 2)$ ) et la proposition IV 1 de [1], nous pouvons supposer que $\mu$ est apériodique. Si les élément de $\left\{\rho_{\lambda}, \lambda \in \Lambda_{0}\right\}$ sont $\mu$-positifs, nous avons alors $M_{-}^{\mu}=\{e\} ;$ d'âprès la proposition (10.1), les G-espacés $\Pi_{\mu}$ et $\Pi_{\xi(\mu)}$ sont G-homéomorphes.

Supposons que $\mu$ possède un moment d'ordre 1 . Nous savons que $\tilde{\Lambda}_{\mu}$, (lemme (11.4)) est wne version de l'espace de Poisson de $\mu$. D'après (10.4), $\Lambda_{\mu}$ est compact si et seulement si nous avons :

i) $M_{-}^{*}=\{e\}$;

ii) la chaîne de loi $\mu$ sur $K$ possède un nombre fini de classes ergodiques. Or la première (resp. la deuxième) condition est satisfaite si et seulement şi les $\rho_{\lambda}, \lambda \in \Lambda_{0}$, sont $\mu$ ت̈positifs (resp. $Z\left({ }_{R} l^{G}\right) \cap T_{\xi(\mu)} T_{\xi(\mu)}^{-1}$ est d'indice fini dans 
le centre $Z\left(R \backslash^{G}\right)$ de $R l^{G}$, (voir [24])). D'autre part si $\Lambda_{\mu} n^{\prime}$ 'est pas compact, du lemme $(11.6)$ il s'ensuit que $\Pi_{\mu}$, dont une version est $\tilde{\Lambda}_{\mu}$, n'est ni homogène ni métrisable.

11.8. Démonstration de la proposition (8.7). - Soient $(G, \mu)$ vérifiant les hypothèses du théorème $(8.4)$ et $\left(\Lambda_{\mu}, \nu\right)$ la $\mu$-frontière homogène de $G$ associée à $(G, \mu)$ par ce théorème. Soit $(E, \lambda)$ une quelconque $\mu$-frontière homogène de $G$. Pour tout $f \in C_{u}(E)$, $f * \lambda$ est un élément de $H_{\mu}$; notons $j(f)$ l'unique élément de $C_{u}\left(\Lambda_{\mu}\right)$ tel que

$$
f * \lambda=j(f) * v ;
$$

on définit ainsi une application linéaire $j$ de $C_{U}(E)$ dans $C_{u}\left(\Lambda_{\mu}\right)$, commutant avec les opérations de $G$.

Désignons par $Z$ (resp. Z') la variable aléatoire à valeurs dans $\Lambda_{\mu}$ (resp. dans $E$ ) telle que $x_{n} \cdot v$ (resp. $x_{n} \cdot \lambda$ ), converge vaguement, $\mathbb{P}_{x}-p \cdot x \cdot \forall x \in G$, vers $\varepsilon_{Z}$ (resp. $\varepsilon_{Z}{ }^{\prime}$ ). Pour tout $g \in G$, nous avons, $\mathbb{P}_{x}$-p.s. $\forall x \in G$,

et

$$
f\left(g \cdot Z^{\prime}\right),=f^{g}\left(Z^{\prime}\right)=\lim _{n} f^{g} * \lambda\left(x_{n}\right)
$$

$$
j(f)(g \cdot z)=j\left(f^{g}\right)(z)=\lim _{n} j\left(f^{g}\right) * v\left(x_{n}\right) .
$$

Mais $G$ étant séparable et les fonctions $f$ et $j(f)$ appartenant respectivement à $C_{u}(E)$ et $C_{u}\left(\Lambda_{\mu}\right)$, nous avons, $\mathbb{P}_{x}$-p.s. $\forall x \in G$,

$$
f\left(g \cdot Z^{\prime}\right)=\lim _{n} f^{g} * \lambda\left(x_{n}\right), \quad \forall g \in G
$$

et

$$
j(f)(g \cdot Z)=\lim _{n} j\left(f^{g}\right) * v\left(X_{n}\right), \quad \forall g \in G ;
$$

D' où, $\mathbb{P}_{x}$-p.s. $\forall x \in G$,

$$
\text { (*) } \quad f\left(g \cdot Z^{\prime}\right)=j(f)(g \cdot Z), V g \in G \text {. }
$$

$\Lambda_{\mu}$ et $E$ étant homogènes, de (*) il résulte que j conserve la norme et vérifie

$$
j\left(f_{1} f_{2}\right)=j\left(f_{1}\right) j\left(f_{2}\right) \quad f_{1}, f_{2} \in C_{u}(E) .
$$

$j$ est donc un isomorphisme d'algèbre de $C_{U}(E)$ sur son image.

Désignons par $\left(\tilde{\Lambda}_{\mu}, \varepsilon\right)$ (resp. $(\tilde{E}, \eta)$ ) une G-compactification de $\Lambda_{\mu}$ (resp. de E), (déf. (11.1)), $\tilde{\Lambda}_{\mu}$ et $\varepsilon(\nu)$ sont respectivement des versions de l'espace de Poisson et du noyau de Poisson de $\mu \cdot(\tilde{E}, \eta(\lambda))$ est une $\mu$-frontière compacte de $G$. D'après ce qui précède, l'application qui à $f \in C(\tilde{E})$ associe l'unique élément $\tilde{j}(f) \in C\left(\tilde{\Lambda}_{\mu}\right)$ tel que

$$
f * \eta(\lambda)=\tilde{j}(f) * \varepsilon(v) \text {, }
$$


est un isomorphisme d'algèbre de $C(\tilde{E})$ sur son image. L'application duale de $\tilde{j}$ envoie alors les caractères de $C\left(\tilde{\Lambda}_{\mu}\right)$ sur ceux de $C(\tilde{E})$; il existe donc une application continue $\tilde{\phi}$, de $\tilde{\Lambda}_{\mu}$ dans $\tilde{E}$, commutant avec les opérations de $G$, telle que $\tilde{j}(f)=f \circ \tilde{\phi}$, pour tout élément $f$ de $C(\tilde{E}$ ). 'La relation (**) s'écrit alors

$$
f * n(\lambda)=f \circ \tilde{\phi} * \varepsilon(\nu)=f * \tilde{\phi}(\varepsilon(\nu)) ;
$$

ce qui montre que $\tilde{\phi}$ envoie $\varepsilon(\nu)$ sur $n(\lambda)$.

Comme $\tilde{\phi}$ envoie $\varepsilon(\nu)$ sur $\eta(\lambda)$, nous avons $\tilde{\phi}\left(\varepsilon\left(\Lambda_{\mu}\right) \cap \eta(E) \neq \emptyset\right.$; par suite, $\Lambda_{\mu}$ et $E$ étant homogènes, nous avons $\tilde{\phi}\left(\varepsilon\left(\Lambda_{\mu}\right)\right)=n(E)$ et l'application

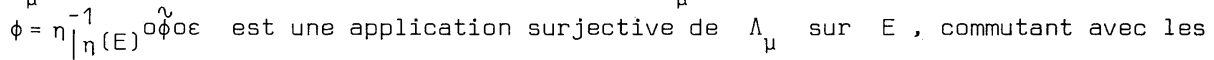
opérations de $G$ et envoyant $\nu$ sur $\lambda$. La proposition (8.7) est prouvée. 
G désigne un groupe de Lie ayant un nombre fini de composantes connexes. Nous reprenons les notations du paragraphe 1 .

12.1. Famille discriminante d'homomorphismes associée à G. - Appelons $\Delta$ l'ensemble des poids de la représentation adjointe du radical $\mathcal{R}$ de $\boldsymbol{g}$ dans le nilradical $\mu^{\mathbb{C}}$ de $\mathfrak{R}^{\mathbb{C}}$. Par un raisonnement en tout point semblable à celui du lemme (2.8), on montre que pour tout automorphisme $\theta$ de $f$ et tout $\alpha$ de $\Delta$, $\alpha \circ \theta \in \Delta$. En particulier $\alpha \circ$ Ad $g=\alpha$, pour tout $\alpha \in \Delta$ et tout $g \in G_{0}$.

Tout élément $\alpha$ de $\Delta$ est la différentielle en e d'un poids $\phi_{\alpha}$ de la représentation adjointe du radical $R$ de $G$ dans $\mathcal{H}^{\mathbb{d}}$. Nous avons alors :

i) Pour tout automorphisme $\sigma$ de $G_{0}, \phi_{\alpha} \circ \sigma=\phi_{\alpha o d \sigma}$. En particulier $\phi_{\alpha}\left(g g^{-1}\right)=\phi_{\alpha}(r) \quad\left(g \in G_{0}, r \in R\right)$.

ii) $\phi_{\alpha}$ prend la valeur 1 pour tout élément du nilradical de $R$.

Soit $G_{0}=R S$ une décomposition de Lévi de $G_{0}$, composante connexe de l'unité dans $G$. Posons alors $\phi_{\alpha}(g)=\phi_{\alpha}(r)$, pour $g=r s \in G_{0}$ avec $r \in R$ et $s \in S$. Il est facile de vérifier (utilisant i) et il)) que l'on définit ainsi un homomorphisme continu $\phi_{\alpha}$ de $G_{0}$ dans $\mathbb{C}$ multiplicatif. En outre un petit calcul utilisant le théorème de Lévi-Malcev, montre que cet homomorphisme est indépendant du choix du sous-groupe de Lévi $S$.

A tout $\alpha \in \Delta$, on a donc associé un homomorphisme $\phi_{\alpha}$ de $G_{0}$ dans $\mathbb{C}$ multiplicatif.

$G$ possède des sous-groupes compacts maximaux deux à deux conjugués et si $L$ est l'un quelconque d'entr'eux nous avons $G=G_{0} L$. Choisissons un tel sous-groupe $L$ de $G$ et posons pour $g=g_{0} l \in G$, avec $g_{0} \in G_{0}$ et $l \in L$,

$$
\begin{aligned}
\Psi_{\alpha}(g) & =\int_{L} \log \left|\phi_{\alpha \circ A d l}\left(g_{0}\right)\right| d l \\
& =\int_{L} \log \left|\phi_{\alpha}\left(\lg _{0} l^{-1}\right)\right| d l,
\end{aligned}
$$

d'où dl est la mesure de Haar normalisée sur $L$.

On vérifie que l'on définit ainsi un homomorphisme $\Psi_{\alpha}$ de $G$ dans $\mathbb{R}$ additif indépendant du choix du sous-groupe compact $L$. En outre, si s désigne une section, à valeurs dans $L$, de l'application naturelle $\zeta$ de $G$ sur $G_{0}^{G}$, on voit
facilement que l'on a 


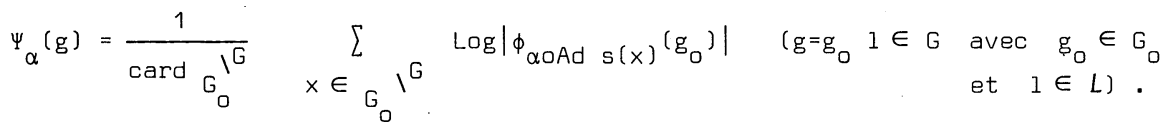

12.2. - Soit $\Delta_{0}$ le sous ensemble de $\Delta$ formé des poids de la représentation adjointe de $\boldsymbol{R}$ dans $(\mu /[\mu, \mu])^{\mathbb{C}}$. Tout élément $\alpha$ de $\Delta$ est alors une somme d'éléments de $\Delta_{0}$. Il s'ensuit que tout élément de la famille d'homomorphismes $\left\{\Psi_{\alpha}, \alpha \in \Delta\right\}$ associée à $G$ est une somme d'éléments de $\left\{\Psi_{\alpha}, \alpha \in \Delta_{0}\right\} .\left\{\Psi_{\alpha}, \alpha \in \Delta_{0}\right\}$ sera appelée la famille discriminante restreinte d'homomorphismes associée à G .

12.3. - Appelons $\Lambda$ (resp. $\Sigma_{1}$ ) l'ensemble des poids de la représentation adjointe de $D$ dans $\mu^{\mathbb{C}}$ (resp. dans $\left.(\boldsymbol{h} / \boldsymbol{\mu})^{\mathbb{C}}\right) \cdot(\operatorname{Voir}(5.5))$.

Soit $R^{\prime}$ le plus grand sous-groupe résoluble distingué de $G_{0} ; R^{\prime} I^{G_{0}}$ est un groupe de Lie semi-simple connexe de centre trivial. Nous notons $\xi^{\prime}$ l'application naturelle de $G$ sur $R^{\prime} I^{G}$ et $\left(I, \xi^{\prime}\right)$ l'application qui au couple (u,g) de $B(G) \times G$ associe le couple $\left(u, \xi^{\prime}(g)\right)$ de $B(G) \times R^{\prime} I^{G}$.

Nous avons :

12.4. Proposition. - La famille $\left\{\rho_{\lambda}, \lambda \in \Sigma_{1}\right\} \quad n^{\prime}$ 'est autre que l'ensemble des composés par l'application $\left(I, \xi^{\prime}\right)$ de $i_{a}$ famille $d^{\prime} A$-cocycles sur $\mathrm{B}(\mathrm{G})$ associée au groupe de Lie $\mathrm{R}^{\mathrm{G}}$ (voir (2.9)).

Tout A-cocycle $\rho_{\lambda}, \lambda \in \Lambda$, est la somme d'un homomorphisme $\Psi_{\alpha}, \alpha \in \Delta$, (voir (12.1)) et du composé par (I, $\left.\xi^{\prime}\right)$ d'un A-cocycle sur $B(G) \times{ }_{R}, I^{G}$ (qui n'appartient pas nécessairement à la famille d'A-cocycles sur $B(G)$ associée à $\left.R^{\prime} I^{G}\right)$. Les deux familles $\left\{\rho_{\lambda}, \lambda \in \Lambda\right\}$ et $\left\{\Psi_{\alpha}, \alpha \in \Delta\right\}$ coincident si et seulement si l'algèbre de Lie $\boldsymbol{g}$ de $\mathrm{G}$ est le produit direct d'une algèbre semi-simple et d'une algèbre moyennable (i.e. extension compacte de son radical).

Preuve. - Nous reprenons les notations antérieures. $R^{\prime} \cap S$ est un sous-groupe résoluble distingué du groupe semi-simple connexe $S$, par suite $R^{\prime} \cap S$ est discret et est donc contenu dans le centre $Z(S)$ de $S$.

Comme $Z(S) \subset \Gamma \subset K$, il s'ensuit que $R^{\prime} \cap N A=\{e\}$ et la restriction, $\xi^{\prime} \mid N A$, de $\xi^{\prime}$ '̀ $N A$ est un homéomorphisme de $N A$ sur son image. D'autre part $R^{\prime}{ }^{G}=\xi^{\prime} \cdot(N) \xi^{\prime}(A) \xi^{\prime}(K)$ est une décomposition d'Iwasawa de $R^{\prime}{ }^{G}$ et il est facile de voir que le normalisateur de $\xi^{\prime}(N A)$ dans $R^{\prime^{G}} n^{\prime}$ est autre que $\xi^{\prime}\left(N A \Gamma^{\prime}\right)$.

Soit $\lambda \in \Xi ; \lambda$ est la différentielle en $e$ d.un poids $\phi_{\lambda}$ de la représentation adjointe de 0 daris $\boldsymbol{h}^{\mathbb{C}}$ vérifiant (voir (2.9)) 


$$
\phi_{\lambda}\left(x d x^{-1}\right)=\phi_{\lambda}(d) \quad\left(d \in D, x \in N A \Gamma^{\prime}\right)
$$

Posons $\overline{\phi_{\lambda}}=\phi_{\lambda} \circ \xi^{\prime-1} \mid$ NA . D'après le lemme (2.4), à l'homomorphisme Log $\left|\overline{\phi_{\lambda}}\right|$ de $R^{\prime} I^{G}$ dans $\mathbb{R}$ additif se trouve associé un $A$-cocycle $\overline{\rho_{\lambda}}$ sur $B(G) \times R^{,} I^{G}$. Comme tout élément de $\Xi$ appartenant à $\Sigma_{1}$ s'identifie à un poids de la représentation adjointe de $\boldsymbol{U}$ dans $\mathcal{P}$, il est clair que $\left\{\overline{\rho_{\lambda}}, \lambda \in \Sigma_{1}\right\}$ n'est autre que la famille d' A-cocycles sur $B(G)$ associée au groupe $R^{\prime} l^{G}$ (voir (2.9)).

D'autre part soit $\lambda$ un élément de $\Xi$ appartenant à $\Lambda$. La restriction, $\lambda / \boldsymbol{R}$, de $\lambda$ à $\boldsymbol{R}$ est un poids de la représentation adjointe de $\boldsymbol{R}$ dans $\boldsymbol{\mu}^{\mathbb{C}}$, c'est-à-dire un élément de $\Delta$; notons $\Psi_{\lambda}$ l'homomorphisme qui lui est associé (voir (12.1)). Il est alors clair que l'on a

$$
\rho_{\lambda}=\Psi_{\lambda_{\mathscr{Q}}}+\overline{\rho_{\lambda}} \circ\left(I, \xi^{\prime}\right) .
$$

Comme on sait que $\boldsymbol{a}$ agit de façon semi-simple sur $\boldsymbol{\Omega}$, il est clair que les deux familles $\left\{\rho_{\lambda}, \lambda \in \Lambda\right\}$ et $\left\{\Psi_{\alpha}, \alpha \in \Delta\right\}$ coincident si et seulement si on a $[\boldsymbol{u}, \boldsymbol{M}]=(0)$. Si $\boldsymbol{g}$ est le produit direct d'une algèbre semi-simple et d'une algèbre moyennable, cette condition est satisfaite. Réciproquement supposons que l'on ait $[\boldsymbol{\mu}, \boldsymbol{\mu}]=(0)$. Soit $\boldsymbol{\sigma}$ un facteur simple non compact de $\mathcal{\jmath}$ et coṇsidérons $y=\{x \in \sigma:[x, \mu]=(0)\}$. $\boldsymbol{\gamma}$ est un idéal de $\boldsymbol{\sigma} ;$ comme $\boldsymbol{a} \cap \boldsymbol{\sigma} \neq(0)$ est contenu dans $y$ et $\sigma$ est simple, nous avons $y=\sigma$. Par suite si on écrit $\mathcal{S}=\mathcal{I}_{1} \times \mathcal{I}_{2}$, où $\mathcal{I}_{1}$ est une algèbre de Lie semi-simple compacte et $\mathcal{I}_{2}$ une algèbre de Lie semi-simple sans facteur compact, nous avons $\left[\mathcal{I}_{2}, \mathcal{H}\right]=(0)$ et donc $\left[\mathcal{J}_{2}, \boldsymbol{R}\right]=(0)$. $\boldsymbol{g}$ est alors le produit direct d'une algèbre moyennable $\boldsymbol{S} \boxplus \mathcal{S}_{1}$ et d'une algèbre semi-simple $\mathcal{\rho}_{2}$.

La proposition (12.4)) est prouvée.

12.5. Définitions. - Une partition $\left\{\Lambda_{-}, \Lambda_{+}\right\}$de $\Lambda$ est dite induite par une partition $\left\{\Delta_{-}, \Delta_{+}\right\}$de $\Delta$ si $\Lambda_{-}$(resp. $\Lambda_{+}$) est le sous ensemble de $\Lambda$ formé des éléments $\lambda$ dont la restriction, $\lambda / \mathscr{R}_{\boldsymbol{R}}$, à $\boldsymbol{R}$ appartient à $\Delta_{-}\left(\mathrm{resp} . \Delta_{+}\right)$.

Une partition $\left\{\Delta_{-}, \Delta_{+}\right\}$de $\Delta$ est dite stable par automorphismes intérieurs si pour tout $\alpha \in \Delta_{-}$(resp. $\Delta_{+}$) et tout $g$ de $G, \alpha$ o Ad g $\in \Delta_{-}$(resp. $\Delta_{+}$). Si $G$ est connexe cette condition est toujours satisfaite (car $\alpha \circ \operatorname{Ad} g=\alpha$, $\forall g \in G_{0}, \forall \alpha \in \Delta$, voir $\left.(12.1)\right)$.

12.6. Proposition. - Soit $\left\{\Delta_{-}, \Delta_{+}\right\}$une partition de $\Delta$ stable par addition, conjuguaison et automorphismes intérieurs; notons $\left\{\Lambda_{-}, \Lambda_{+}\right\}$la partition de $\Lambda$ induite par cette partition de $\Delta$. Alors $\left\{\Lambda_{-}, \Lambda_{+} \cup \Sigma_{1}\right\}$ est une partition de $\Xi$ stable par addition et conjuguaison; la décomposition de $G$ associée à cette par- 
tition de $\Xi$ (proposition (4.2)) est une décomposition de $G$ en produit amalgamé de deux sous-groupes.

Preuve. - $\left\{\Lambda_{-}, \Lambda_{+}\right\}$est une partition de $\Lambda$ stable par addition et conjuguai-

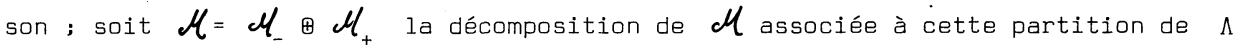
(voir (3.7)). Comme la partition $\left\{\Lambda_{-}, \Lambda_{+}\right\}$est induite par une partition de $\Delta$, d'après le lemme (5.6) il s'ensuit que $\boldsymbol{\mu}_{\text {- }}$ et $\boldsymbol{\mu}_{+}$sont stables par $\mathcal{J}$.

Désignons par $D=D_{-} \oplus D_{+}$et $\boldsymbol{y}=D_{-} \oplus \xi_{+}$les décompositions de $D$ et $\mathcal{f}$ associées à la partition $\left\{\Lambda_{-}, \Lambda_{+} \cup \Sigma_{1}\right\}$ de $\Xi$ (voir (4.1)). Nous avons $D_{-}=\mu_{-}, D_{+}=\left(\mu_{+}+P_{)} \oplus Q^{\oplus} \mathcal{N}\right.$ et $\xi_{+}=\left(\mu_{+} \boldsymbol{S}\right) \oplus \mathcal{S}$. $\xi_{+}$est donc une sous-algèbre de $\boldsymbol{y}$.

Soit $G=D_{-} E_{+}$la décomposition de $G$ associée à la partition $\left\{\Lambda_{-}, \Lambda_{+} \cup \Sigma_{1}\right\}$ (voir proposition (4.2)). Nous avons

$$
D_{-}=\exp \mu_{-} \text {et } E_{+}=\left(\exp \mu_{+}\right) P S \Gamma^{\prime} \text {. }
$$

Comme la partition $\left\{\Delta_{-}, \Delta_{+}\right\}$de $\Delta$ est supposée stable par automorphismes intérieurs, nous avons (lemme (5.6)) Ad k $\left(\mu_{-}\right) \subset \mu_{-}$et Ad $k\left(\mu_{+}\right) \subset \mu_{+}$pour tout $k \in \Gamma^{\prime}$. Par suite $\Gamma^{\prime}$ normalise les sous-groupes exp $\mu_{-}$et exp $\mu_{+}$de $G$. On en déduit que $E_{+}$est un sous-groupe de $G$; $G$ est le produit amalgamé des sous-groupes $G_{-}$et $E_{+}$(proposition (4.2)).

On notera que si $y$ est le produit direct d'une algèbre de Lie moyennable et d'une algèbre de Lie semi-simple, toute partition $\left\{\Lambda_{-}, \Lambda_{+}\right\}$de $\Lambda$ est induite par une partition de $\Delta$.

12.7. Proposition. - Soient G un groupe L.C.D. et $\Psi$ un homomorphisme continu de $G$ dans $\mathbb{R}$ additif. Soit $\mu$. une mesure $M^{1}(G)$ (non nécessairement étalée) et désignons par $\left(\Omega, \mathcal{F},\left(x_{n}\right)_{n \in \mathbb{N}},\left(\mathbb{P}_{g}\right)_{g \in G}\right)$ Za marche aléatoire droite de Zoi $\mu$ sur $G$.

Alors si la mesure $\Psi(\mu)$ n'est pas la mesure de Dirac au point zéro de $\mathbb{R}$ on a l'alternative suivante:

ou bien, $\Psi\left(x_{n}\right)$ converge vers $(-\infty), \mathbb{P}_{g}$-p.s. $\forall g \in G$; ou bien, $\Psi\left(x_{n}\right)$ converge vers $(+\infty), \mathbb{P}_{g}$-p.s. $\forall g \in G$; ou bien, $\lim _{n} \sup \psi\left(x_{n}\right)=+\infty$ et $\lim _{n} \inf \Psi\left(x_{n}\right)=-\infty, \mathbb{P}_{g}-p \cdot s . \quad \forall g \in G$.

Si $\mu$ possède en outre un moment d'ordre 1 , alors nous sommes respectivement dans le premier, deuxième ou troisième cas suivant que $\int_{G} \Psi(g) \mu(d g)$ est négatif strict, positif strict, ou nut.

Preuve. - Le résultat énoncé ci-dessus est bien connu. Dans le cas où $\mu$ est 
étalée il se déduit des propositions (7.8) et (7.12). Dans le cas où $\mu$ n'est pas étalée, une démonstration analogue à celle de la proposition (7.8), faisant toutefois appel au théorème de. Choquet-Deny sur les fonctions harmoniques sur les groupes abéliens, permet de prouver la première assertion de la proposition (12.6). D'autre part si $\mu$ possède un moment d'ordre 1, d'après la loi des grands nombres $\frac{1}{n} \Psi\left(x_{n}\right)$ converge, $\mathbb{P}_{g}$-p.s. $\forall g \in G$, vers $\alpha=\int_{G} \Psi(g) \mu(d g)$. Si $\alpha<0$ (resp. $\alpha>0)$, on est alors dans le premier (resp. le deuxième) cas. Si $\alpha=0$, on sait (voir par exemple [23]) que la marche aléatoire sur $\mathbb{R}, \psi\left(x_{n}\right)$, est récurrente (i.e. revient une infịnité de fois, $\mathbb{P}_{\mathrm{g}}$-p.s. $\forall \mathrm{g} \in \mathrm{G}$, dans tout ouvert de $\mathbb{R}$ ). On est alors dans le troisième cas.

Dorénavant $G$ désigne un groupe de Lie ayant un nombre fini de composantes connexes dont l'algèbre de Lie $\boldsymbol{f}$ est le produit direct d'une algèbre de Lie moyennable et d'une algèbre de Lie semi-simple. Les deux familles $\left\{\rho_{\lambda}, \lambda \in \Lambda\right\}$ et $\left\{\Psi_{\alpha}, \alpha \in \Delta\right\}$ coincident donc.

12.8. Décomposition de $G$ associée à une mesure $\mu$ de $M^{1}(G)$. - Posons

et

$$
\begin{aligned}
& \Delta_{-}^{\mu}=\left\{\alpha \in \Delta: \Psi_{\alpha} \text { est } \mu \text {-négatif strict }\right\} \\
& \Delta_{+}^{\mu}=\left\{\alpha \in \Delta: \Psi_{\alpha} \text { est } \mu \text {-positif }\right\} .
\end{aligned}
$$

La partition $\left\{\Delta_{-}^{\mu}, \Delta_{+}^{\mu}\right\}$ de $\Delta$ est stable par addition, conjuguaison et automorphisme intérieur (définition (12.5)).

Si $\mu$ possède un moment d'ordre 1 nous avons

$$
\begin{aligned}
& \Delta_{-}^{\mu}=\left\{\alpha \in \Delta: \int_{G} \Psi_{\alpha}(g) \mu(d g)<0\right\} \\
& \Delta_{+}^{\mu}=\left\{\alpha \in \Delta: \int_{G} \Psi_{\alpha}(g) \mu(d g) \geqslant 0\right\}
\end{aligned}
$$

A toute mesure $\mu \in M^{1}(G)$ se trouve donc associée (voir proposition (12.6)) une décomposition de $G$ en produit amalgamé de deux sous-groupes. Nous la noterons $G=M_{-}^{\mu} T_{+}^{\mu}$.

Nous avons alors:

i) Pour toute mesure $\mu$ de $M^{1}(G)$ apériodique, possèdant un moment d'ordre 1 (non nécessairement étalée), la composante de $x_{n} g$ sur $M_{-}^{\mu}$ converge, $\mathbb{P}_{x}-p . s . \forall x \in G$, et la limite est indépendante de $\mathrm{g} \in \mathrm{G}$. [Comme $\mathcal{H}_{-}$et $\boldsymbol{\mu}_{+}$sont stables par $\mathcal{\rho}$, i) résulte du théorème (9.1) dans le cas où $\mu$ est étalée; en fait, cette dernière condition n’est pas nécessaire car nous avons

$$
\alpha \in \Delta_{-}^{\mu} \Longleftrightarrow \int_{G} \Psi_{\alpha}(g) \mu(d g)<0,
$$


sans pour cela supposer que $\mu$ est étalée. (Voir proposition (12.7))].

D'autre part nous avons :

12.9. Théorème. - Soit $G$ un groupe de Lie ayant un nombre fini de composantes connexes qui est localement le produit direct d'un groupe de Lie moyennable et d'un groupe de Lie semi-simple. Soient $\mu$ une mesure de $M^{1}(\mathrm{G})$ étalée, apériodique, possèdant un moment d'ordre 1 et $G=M_{-}^{\mu} T_{+}^{\mu}$ une décomposition, en produit amalgamé de sous groupes, associée à $\mu$ (voir (12.8)). Alors $\Lambda_{\mu}(G)$ (voir théorème (8.4)) est en tant que G-espace le produit direct des G-espaces $G_{T_{+}^{\mu}} \times \Lambda_{\xi(\mu)}\left({ }_{R}{ }^{G}\right)$, où $R$ désigne le radical de la composante connexe $G_{0} d e$ Z'unité dans $G$ et $\xi l^{\prime a p p l i c a t i o n ~ n a t u r e z l e ~ d e ~} G$ sur $\mathrm{R}^{\mathrm{G}}$. En outre si $\left.\left(\Omega, \mathscr{F}_{,}\left(\mathrm{X}_{n}\right)_{n \in \mathbb{N}}, \mathbb{P}_{\mathrm{g}}\right)_{\mathrm{g} \in \mathrm{G}}\right)^{\prime}$ désigne la marche aléatoire droite de Zoi $\mu$ sur $G$, pour tout $x \in G T_{+}^{\mu}, x_{n} \times$ converge, $\mathbb{P}_{g}-p . s . \forall g \in G$, et la v.a. limite ne dépend pas de $x$.

En particulier si $G$ est moyennable (i.e. $\mathrm{R}^{\mathrm{G}}$ est compact), nous avons $\Lambda_{\mu}(G)=G / T_{+}^{\mu}$ et $v$ (voir théorème (8.4)) est l'unique mesure de probabilité $\mu$-invariantè sur $\Lambda_{\mu}(G)$.

Preuve. - Le fait que $x_{n} \cdot x$ converge, $\mathbb{P}_{g}-$ p.s. $\forall g \in G$, pour tout élément $x$ de ${ }^{G} T_{+}^{\mu}$ et que cette limite soit indépendante de $x$ est une conséquence de $i$ ).

Reprenons les notations antérieures. Nous avons

$$
T_{+}^{\mu}=M_{+}^{\mu} P S \Gamma^{\prime} \text { et } S=N A N \Gamma \text { (modulo } m_{S} \text { ) . }
$$

Nous savons (voir $(8.5))$ que $\Lambda_{\mu}(G)={ }^{G} / M_{+}^{\mu} P_{P A N \Gamma_{\mu}^{\prime}}$, où $\Gamma_{\mu}^{\prime}$ est un sous groupe de $\Gamma^{\prime}$ contenant $\Gamma_{0}^{\prime}$ et que $\Lambda_{\xi(\mu)}\left({ }_{R}{ }^{G}\right)=\xi(G) /_{\xi\left(A N \Gamma_{\mu}^{\prime}\right)}$.

Des hypothèses faites sur $G$, il résulte que les sous groupes fermés $M_{-}^{\mu}$ et $M_{+}^{\mu}$

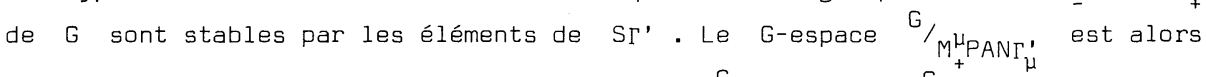
G-homéomorphe au produit direct des G-espaces $G /_{T_{+}^{\mu}} \times \Lambda_{\xi(\mu)}\left({ }_{R} l^{G}\right)^{+}$.

Si $G$ est moyennable, $R^{G}$ est compact ; comme $\mu$ est apériodique, il s'ensuit que $\left.\Lambda_{\xi(\mu)}(R)^{G}\right)$ est réduit à un point et donc $\Lambda_{\mu}={ }^{G} / T_{+}^{\mu}$. Soient $\nu^{\prime}$ une mesure $\mu$-invariante de $M^{1}\left(\Lambda_{\mu}\right)$ et $f$ une fonction continue à süpport compact sur $\Lambda_{\mu}$; la fonction $f * v^{\prime}$ est $\mu$-harmonique bornée et il s'ensuit que $f * v^{\prime}\left(x_{n}\right)$ converge, $\mathbb{P}_{\mathrm{g}}$-p.s.. $\forall \mathrm{g} \in \mathrm{G}$, quand $\mathrm{n}$ tend vers l'infini. Mais

$$
f * v^{\prime}\left(x_{n}\right)=\int_{\Lambda_{\mu}} f\left(x_{n} \cdot x\right) v^{\prime}(d x)
$$


et on sait que $X_{n} \cdot x$ converge, $\mathbb{P}_{g}$-p.s. $\forall g \in G$, vers une variable aléatoire de loi $v$, par rapport à $\left(\Omega, \mathscr{F}, \mathbb{P}_{e}\right)^{\prime}$, indépendante de $x \in \Lambda_{\mu}$. D'après le théorème de convergence dominé il s'ensuit alors que

$$
v^{\prime}(f)=\mathbb{E}_{e}\left[f * v^{\prime}\left(X_{n}\right)\right] \rightarrow v(f) ;
$$

ce qui montre que $\nu^{\prime}=\nu$; il existe donc sur $\Lambda_{\mu}$ une unique mesure de probabilité $\mu$-invariante. Le théorème $(12.9)$ est démontré.

Nous obtenons pour ces groupes la caractérisation :

12.10. Corollaire. - Soit G un groupe de Lie ayant un nombre fini de composantes connexes tei que $\mathrm{R}^{G}$ soit de centre fini. Soit $\left\{\Psi_{\alpha}, \alpha \in \Delta_{0}\right\}$ la famizle restreinte d'homomorphismes de $G$ dans $\mathbb{R}$ additif associée à $G$ (voir (12.1)). Soit $\mu$ une mesure de $M^{1}(G)$ étalée, possèdant un moment d'ordre 1 et vérifiant

$$
\int_{G} \Psi_{\alpha}(g) \mu(d g)=0, \quad \forall \alpha \in \Delta_{0} .
$$

(Ce qui est le cas si $\mu$ eșt symétrique!).

Alors nous avons les équivalences :

i) Z'espace de Poisson $\Pi_{\mu}$ de $\mu$ est métrisable;

ii) $\Pi_{\mu}$ est homogène;

iii) $\Pi_{\mu}$ est G-homéomorphe $\grave{a} \Pi_{\xi(\mu)}$;

iv) l'algèbre de Lie $y$ de $G$ est le produit direct d'une algèbre moyennable et d'une algèbre semi-simple.

Preuve. - D'après la proposition (12.4), la condition iv) de ce corollaire équivaut à :

v) les familles $\left\{\Psi_{\alpha}, \alpha \in \Delta\right\}$. et $\left\{\rho_{\lambda}, \lambda \in \Lambda\right\}$ sont identiques.

Donc si iv) est vérifiée, les éléments $\rho_{\lambda}, \lambda \in \Lambda$, sont tous $\mu$-positifs et $d$ 'après le théorème (11.3) on a donc iv) $\Longrightarrow$ iii).

Comme il est clair que iil) $\Longrightarrow$ iil $\Longrightarrow$ il, il nous faut donc prouver que i) $\Longrightarrow$ iv).

Supposons que iv) ne soit pas vérifiée; c'est-à-dire que l'action adjointe de $\boldsymbol{U}$ dans $\boldsymbol{M}$ soit non triviale (voir (12.4)). Pour $\lambda \in \Lambda$, écrivons

$$
\rho_{\lambda}=\Psi_{\lambda}+\bar{\rho}_{\lambda} \circ\left(I, \xi^{\prime}\right) \quad(\operatorname{voir}(12.4))
$$

et posons

$$
a_{\lambda}=\iint \rho_{\lambda}(u, g) v(d u) \mu(d g) \text { et } \bar{a}_{\lambda}=\iint \bar{\rho}_{\lambda}(u, g) v(d u) \mu(d g) \text {, }
$$

où $\nu$ désigne l'unique mesure de probabilité $\mu$-invariante sur la frontière maximale $B(G)$ de $G$. L'action adjointe de $\mathcal{C}$ sur $\mu$ étant semi-simple, il s'ensuit 
que l'on a

(*)

$$
\lambda \sum_{\Lambda} \bar{a}_{\lambda}=0
$$

D'autre part comme $[\boldsymbol{Q}, \boldsymbol{\mu}] \neq(0)$, il est clair (voir (12.4)) qu'il existe $\gamma \in \Sigma_{1}$ tel que $\left[\mathcal{S}_{\gamma}, \mu\right] \neq(0)$ et par suite il existe des éléments $\lambda_{1}$ et $\lambda_{2}$ de $\Lambda$ tels que

$$
\left.\lambda_{1}\right|_{\Omega}=\left.\lambda_{2}\right|_{\Omega} \text { et }\left.\lambda_{2}\right|_{a}=\left.\lambda_{1}\right|_{a}+r \text {. }
$$

Autrement dit il existe des éléments $\lambda_{1}$ et $\lambda_{2}$ de $\Lambda$ tels que

$(* *)$

$$
\bar{a}_{\lambda_{2}}=\bar{a}_{\lambda_{1}}+a_{\gamma} \cdot
$$

Du fait que $a_{\gamma}<0$ (corollaire (7.15)), il résulte de (*) et (**) qu'il existe des éléments $\lambda$ de $\Lambda$ pour lesquels $\bar{a}_{\lambda}<0$. D'après l'hypothèse $\int_{G} \Psi_{\alpha}(g) \mu(d g)=0, \forall \alpha \in \Delta$, il s'ensuit alors qu'il existe des $A$-cocycles $\rho_{\lambda}, \lambda \in \Lambda$, qui sont $\mu$-négatifs stricts. D'après le théorème (11.3), i) n'est donc pas vérifié.

Nous retrouvons donc le résultat de [4] :

12.11. Corollaire. - Soit G un groupe de Lie ayant un nombre fini de composantes connexes tel que $\mathrm{R}^{\mathrm{G}}$ soit de centre fini. Alors $\mathrm{G}$ est de type (1) (i.e. l'espace de Poisson $\pi_{\mu}$ est homogène pour toute mesure de $M^{1}(G)$ étalée; voir [1]) si et seulement si l'algèbre de Lie de G est le produit direct d'une algèbre semi-simple et d'une algèbre moyennable telle que les valeurs propres de adX soient imaginaires pures pour tout élément $x$ de son radical. 
13.1. Lemme. - Tout groupe L.C.D. tel que $G_{0} \backslash^{G}$ soit compact possède une frontière maximale $\mathrm{B}(\mathrm{G})$ unique à G-homéomorphisme près. Tout sous groupe compact distingué de $G$ opère trivialement sur $B(G)$.

Preuve. - On sait ([21]) qu'il existe des sous groupes compacts distingués, C, arbitrairement petits tels que $C^{G}$ soit un groupe de Lie ayant un nombre fini de composantes connexes. Pour un tel groupe $C$ de $G$; notons $\left.B\left(C_{C}^{G}\right)=H_{C} l^{G}\right)^{G}$, la frontière maximale de $C^{I^{G}}$. $H\left(C^{l^{G}}\right)$ possède la propriété de point fixe. Soit $H(G)$, l'image réciproque de $H\left({ }_{C}{ }^{G}\right)$ par l'application naturelle de $G$

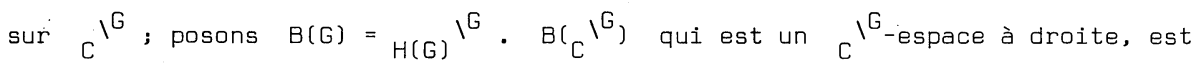
aussi, de façon évidente, un G-espace à droite, G-homéomorphe à $B(G)$. D'après ([1] Jemme II.8), H(G) possède la propriété de point fixe. Par suite, ([1], corollaire 1 de la proposition II.2), B(G) est une frontière maximale de $G$ et toute frontière maximale de $G$ est $G$-homéomorphe à $B(G)$. Il s'ensuit d'une part que $B\left(C^{G}\right.$ ) est indépendant (à G-homéomorphisme près) du choix du sous groupe compact distingué $C$ et d'autre part que tout sous groupe compact distingué de G opère trivialement sur toute frontière maximale de G . Le lemme (13.1) est prouvé.

13.2. Lemme. - Soient G un groupe de Lie ayant un nombre fini de composantes connexes; C un sous groupe compact distingué de $\mathrm{G}$ et $\mathrm{P}$ l'application natureile de $\mathrm{G}$ sur $\mathrm{C}^{\mathrm{G}}$. Alors l'application

$$
\left\{\rho_{\lambda}, \lambda \in \Xi \quad\left(\operatorname{resp} . \Lambda_{0}\right)\right\} \longmapsto\left\{\rho_{\lambda} \circ(I, p), \lambda \in \Xi \quad\left(\operatorname{resp} . \Lambda_{0}\right)\right\},
$$

établit une correspondance biunivoque entre les familles discriminantes (resp. discriminantes restreintes) $d^{\prime} A$-cocycles sur $B(G)$ associées à ${ }_{C}{ }^{G}$, et celles associées à G. De plus si $\left\{\Psi_{\alpha}, \alpha \in \Delta\right.$ (resp. $\left.\left.\Delta_{0}\right)\right\}$ désigne la famille discriminante restreinte) d'homomorphismes associée à ${ }_{C}{ }^{G}$, alors celle associée à $G$ est $\left\{\Psi_{\alpha} \mathrm{op}, \alpha \in \Delta\right.$ (resp. $\left.\left.\Delta_{0}\right)\right\}$.

Preuve. - Reprenons les notations du paragraphe 2. Considérons la décomposition $\boldsymbol{y}=\boldsymbol{D} \mathbb{X}$ et notons $\boldsymbol{y}$ le nilradical de $\boldsymbol{D}$.

Soit $\boldsymbol{\zeta}$ l'algèbre de Lie de $c$; $\boldsymbol{\zeta}$ est un idéal de $\boldsymbol{H}$. Désignons par dp la différentielle en e de l'application naturelle $P$ de $G$ sur $C^{G}$.

Notons $\Xi$ (resp. $\bar{\Xi}$ ) l'ensemble des poids de la représentation adjointe de $\varnothing$ 
dans $\boldsymbol{h}^{\mathbb{C}}$ (resp. de $d p(D)$ dans $(\mathrm{dp}(\xi))^{\mathbb{C}}$. Nous allons montrer que

$$
\{\lambda \in \Xi: \operatorname{Re} \lambda \neq 0\}=\{\bar{\lambda} \text { odp }, \bar{\lambda} \in \bar{\Xi}\}
$$

Soit $\lambda$ un élément de $\Xi$. Il existe alors un idéal $W$ de $D$ contenu dans $h$ et un élément $H$ de $h-W$ tels que, tout $x$ de $D$,

$$
[x, H]=\lambda(x) \cdot H \bmod \cdot \omega \text {. }
$$

Si $\operatorname{Re} \lambda \neq 0, H$ ne peut appartenir à $(\boldsymbol{\xi}+\boldsymbol{w}) \cap \boldsymbol{\xi}=\boldsymbol{\xi} \cap \boldsymbol{\xi}+\boldsymbol{w}$. En effet supposons que $H$ appartienne à $\zeta \cap \xi+w$ et écrivons $H=H_{1}+H_{2}$ avec $H_{1} \in \xi \cap \xi$ et $H_{2} \in W^{*}$; de la relation (*) il résulte que $\forall x \in D,\left[X, H_{1}\right]=\lambda(x) \cdot H_{1} \bmod \cdot \omega ;$

et par suite

$$
\forall X \in D, A d \exp H_{1}(X)=\operatorname{Exp} \text { ad } H_{1}(X)=e^{\lambda(X)} \cdot H_{1} \bmod \cdot \boldsymbol{w} ;
$$

Comme Ad exp $H_{1}$ est un élément du groupe compact Ad $C$, on a alors nécessairement $\left|e^{\lambda(x)}\right|=1$, pour tout $x \in D$ (i.e. $\operatorname{Re} \lambda=0$ ). Nous avons alors, pour tout $x$ de $D$,

$$
[d p(X), d p(H)]=d p([X, H])=\lambda(X) \cdot d p(H) \quad \bmod \cdot d p(\boldsymbol{W}),
$$

où $d p(H)$ est un élément de $d p(\boldsymbol{\xi})-d p(\boldsymbol{W})$.

On en déduit que $\lambda$ s'écrit $\bar{\lambda}$ odp, où $\bar{\lambda}$ est un élément de $\bar{\Xi}$.

Inversement soit $\bar{\lambda}$ un élément de $\bar{\Xi}$. Il existe un idéal $\overline{\boldsymbol{w}}$ de dp(D) contenu dans $d p(\xi)$ et un élément $d p(H)$ de $d p(\xi)-\overline{\boldsymbol{w}}$ tels que, pour tout $x$ de $D$,

$$
[d p(X), d p(H)]=\bar{\lambda}(d p(X)) \cdot d p(H) \quad \bmod . \overline{\boldsymbol{w}},
$$

il s'ensuit que l'on a, pour tout $x$ de $\infty$,

$$
[X, H]=\bar{\lambda} \operatorname{odp}(X) \cdot H \quad \bmod \cdot \omega,
$$

où $\boldsymbol{w}=d p^{-1}(\bar{w}) \cap \xi$ est un idéal de $D$ contenu dans $\xi$ et $H$ est un élément de $\zeta-w$. Autrement dit $\bar{\lambda}$ odp appartient à $\Xi$.

La première assertion du lemme (13.2) est alors claire. La deuxième assertion se montre de la même façon.

13.3. Familles discriminantes et discriminantes restreintes d'A-cocycles associées à G. - Soit $C$ un sous groupe compact distingué de $G$ tel que le quotient $C^{G}$ soit un groupe de Lie ayant un nombre fini de composantes connexes. Comme C opère trivialement sur $B(G)$ (lemme (12.1)), l'application $\rho \longmapsto \rho(I, p)$, où $P$ désigne l'application naturelle de $G$ sur $C^{G}$, établit une correspondance biunivoque entre les $A$-cocycles sur $B(G) \times{ }_{C}{ }^{G}$ et les A-cocycles, C-invariants, sur $B(G) \times G$. Nous appelons alors famille discriminante (resp. discriminante res- 
treinte) d'A-cocycles associée à G, l'image par cette application d'une quelconque famille discriminante (resp. discriminante restreinte) d'A-cocycles sur $B(G)$ associée à $C^{G}$. D'après le lemme (13.2), cette définition est indépendante du choix du sous groupe compact distingué C. D'après le lemme (2.12) si $\left\{\rho_{\lambda}, \lambda \in \Xi \quad\right.$ (resp. $\Lambda_{0}^{j}$ \} est une famille discriminante (resp. discriminante restreinte) d'A-cocycle associée à $G$, toute autre famille discriminante (resp. discriminante restreinte) s'écrit $\left\{\rho_{\lambda} \circ\left(J_{x}, I_{x}\right), \lambda \in \Xi\right.$ (resp. $\Lambda_{0}$ ) \}, pour un certain élément $x$ de $G_{0}$, où

$$
\begin{aligned}
& J_{x}: B(G) \rightarrow B(G), I_{x}: G \rightarrow G \\
& u \longmapsto u \cdot x \quad g \longmapsto x^{-1} g x
\end{aligned}
$$

13.4. Théorème. - Soit G un groupe L.C.D. tel que $\mathrm{G}^{\mathrm{G}}$ soit compact. Alors avec les mêmes hypothèses sur la mesure $\mu$ de $M^{1}(G)$, les théorèmes (8.4) et (11.3) s'appliquent au couple $(G, \mu)$.

Preuve. - Soit $S_{\mu}$ le semi-groupe, ouvert associé à $\mu$ (voir (6.2)). $S_{\mu} S_{\mu}^{-1}$ est un voisinage ouvert de e dans $G$. Considérons un sous-groupe compact distingué $C$ de $G$ contenu dans $S_{\mu} S_{\mu}^{-1}$ tel que $C^{l^{G}}$ soit un groupe de Lie ayant un nombre fini de composantes connexes. D'après le théorème (6.8) les fonctions $\mu$-harmoniques bornées sur $G$ sont les composées par l'application naturelle $P$ de $G$ sur $c^{l^{G}}$ des fonctions $p(\mu)$-harmoniques sur $c^{l^{G}}$. Le théorème (13.4) résulte alors des théorèmes ( 8.4 ) et (13.3) que $l$ 'on applique au couple $\left(C{ }^{G}, p(\mu)\right)$.

13.5. - Soit $G$ un groupe L.C.D. tel que $G_{0} \backslash^{G}$ soit compact. S'il existe un sousgroupe compact distingué $C$ de $G$ tel que $C^{G}$ soit un groupe de Lie dont l'algèbre de Lie $\boldsymbol{y}$ est le produit direct d'une algèbre semi-simple et d'une algèbre moyennable (i.e. extension compacte de son radical $\mathfrak{R}$ ), nous disons que $G$ vérifie l'hypothèse $\left(H_{1}\right)$. Si de plus $y$ est le produit direct d'une algèbre semisimple et d'une algèbre moyennable de type (R) (i.e. que les valeurs propres de ${ }_{\mathbb{R}} X$ sont imaginaires pures pour tout $X \in \mathbb{R}$ ), nous disons que $G$ vérifie 1 'hypothèse $\left(\mathrm{H}_{2}\right)$.

13.6. Famille discriminante et discriminante restreinte d'homomorphismes associée à $G$. - Soit $C$ un sous-groupe compact distingué de $G$ tel que $C^{V^{G}}$ soit un groupe de Lie ayant un nombre fini de composantes connexes. Tout homomorphisme de $G$ dans $\mathbb{R}$ additif s'annulant sur $C$, l'application $\Psi \longrightarrow$ Yop, établit une correspondance biunivoque entre les homomorphismes sur $C^{G}$ et ceux sur $G$. Nous appelons alors famille discriminante (resp. discriminante restreinte) d'homomor- 
phismes associée à G, l'image par cette application, de la famille discriminante (resp: discriminante restreinte) d'homomorphismes associée à $C^{G}$. D'après le lemme (13.2), cette définition est indépendante du choix du sous-groupe compact distingué $C$.

13.7. Proposition. - Soit G un groupe L.C.D. tel que $G^{G}$ soit compact. Alors $G$ vérifie l'hypothèse $\left(H_{1}\right)$ (resp. $\left(H_{2}\right)$ ) si et seulement si toute famizle discriminante restreinte d'A-cocycles associée à $G$ coincide avec la famille discriminante restreinte d'homomorphismes associée à G (resp. est réduite à (0)).

Preuve. - La proposition (13.7) résulte de la proposition (12.4).

Nous obtenons alors pour les groupes vérifiant l'hypothèse $\left(\mathrm{H}_{1}\right)\left(\mathrm{resp}\right.$. $\left.\left(\mathrm{H}_{2}\right)\right)$ la caractérisation :

13.8. Corollaire. - Soit $G$ un groupe L.C.D. tel que $G_{0}{ }^{G}$ soit compact et $R^{G}$ soit de centre fini, où $R$ désigne le radical de $G_{0}$, composante connexe de l'unité dans G. Soit $\left(\left\{\Psi_{\alpha}, \alpha \in \Delta_{0}\right\}, \mu\right)$ vérifiant Z'hypothèse de (12.10). AZors nous avons les équivalences :

i) Z'espace de Poisson $\Pi_{\mu}$ de $\mu$ est métrisable;

ii) $\pi_{\mu}$ est homogène;

iii) $\Pi_{\mu}$ est G-homéomorphe à $\Pi_{\xi(\mu)}$;

iv) $G$ verifie l'hypothèse $\left(H_{1}\right)$.

13.9. Corollaire. - Soit G comme dans le corollaire (13.8). Alors G est de type (T) si et seulement si $G$ vérifie l'hypothèse $\left(\mathrm{H}_{2}\right)$. 
14.0. Introduction. - Soit $G$ un groupe de Lie ayant un nombre fini de composantes connexes dont la composante connexe $G_{0}$ de l'unité est semi-simple de centre quelconque. Soit $\mu$ une mesure de probabilité sur $G$. On se propose d’étudier le comportement asymptotique de la $n^{\text {ième }}$ convolée, $\mu^{n}$, de $\mu$.

Soit $G=N A K$ et $G=N A \tilde{N} \Gamma^{\prime} \bmod . m_{G},\left(\right.$ resp. $G_{0}=N A K$ et $G_{0}=N A N \Gamma \bmod$. $m_{G_{0}}$ ) des décompositions d'Iwasawa et de Bruhat de $G$ (resp. de $G_{0}$ ). Posons $B_{0}=K \cap N A \tilde{N}$ et désignons par $W$ la chambre de weyl qui a servi à déterminer $N$ (voir (1.2)). Alors nous savons (voir [26]) que $m_{G}$-presque tout élément $g$ de $G$ s'écrit de façon unique.

$$
g=x \tilde{a} k \text { avec } \quad x \in B_{0}, \quad \tilde{a} \in \exp W \text { et } k \in K .
$$

Dans le cas d'un groupe de Lie connexe semi-simple de centre fini et d'une mesure $\mu$ absolument continue par rapport à la mesure de Haar, Virtser ([26] ) a étudié le comportement de $\mu^{n}$ dans la représentation

$$
G=B_{0}(\exp W) K \bmod \cdot m_{G} \text {. }
$$

Soit $\left(\Omega, \mathfrak{F},\left(x_{n}\right)_{n \in \mathbb{N}},\left(\mathbb{P}_{g}\right)_{g \in G}\right)$ la marche aléatoire droite de loi $\mu$ sur $G$ et écrivons

$$
x_{n}=x_{n}\left(\exp h_{n}\right) k_{n} \quad \text { avec } x_{n} \in B_{0}, h_{n} \in W \text { et } k_{n} \in k
$$

Virtser montre que pour toute loi $\mathbb{P}_{g}, g \in G, k_{n}$ (resp. $x_{n}$ ) se comporte comme la chaine de loi $\mu$ sur $A N{ }^{G}$ (resp. sur la frontière maximale droite $G / A \tilde{A} \Gamma$ de $G)$ et $h_{n}$ est asymptotiquement gaussienne. De plus pour presque tous les éléments $g$ de $G$, (plus précisément pour les éléments $g$ dont l'image dans AN $G$ appartient à une sous-classe cyclique ou à une classe ergodique sans classe cyclique de la chaine de loi $\mu$ sur $A N{ }^{G}$ ); les v.a. $x_{n}$, $h_{n}$ et $k_{n}$ sont asymptotiquement indépendantes, pour la loi $\mathbb{P}_{\mathrm{g}}$.

Notre étude est faite directement dans la représentation d'Iwasawa. Soit $\mu$ une mesure de $M^{1}(G)$ étalée apériodique et possèdant un moment 2 . Ecrivons

$$
x_{n}=N_{n}\left(\exp H_{n}\right) K_{n} \text { avec } N_{n} \in N, H_{n} \in C \text { et } K_{n} \in K \text {, }
$$

où $Q$ désigne l'algèbre de Lie de $A$. $K_{n}$ n'est autre que la chaine de loi $\mu$ sur $K$ identifié à $A N{ }^{G}$. Nous montrons que : pour tout $x \in G$, la composante $N_{n}$, converge $\mathbb{P}_{x}-p . s$. , vers une v.a. dont la loi est équivalente à la mesure de Haar de $N$; $H_{n}$ est asymptotiquement gaussienne pour toute loi $\mathbb{P}_{g}, g \in G$. De plus pour presque tous les éléments $g$ de $G$, les trois composantes sont asymptotiquement indépendantes, pour la loi $\mathbb{P}_{\mathrm{g}}$. Ce résultat permet de retrouver celui 
de Virtser et de le préciser. En particulier on montre que la composante $x_{n}$ converge dans $B_{0}, \mathbb{P}_{x}-$ p.s. $\forall x \in G$.

14.1. - Soit $G$ un groupe de Lie ayant un nombre fini de composantes connexes dont la composante connexe $G_{0}$ de l'unité est semi-simple. Soit $\mu$ une mesure de $M^{1}(G)$ que nous supposons apériodique (i.e. que le sous-groupe fermé engendré par le support de $\mu$ est égal à $G)$. Soit $\left(\Omega, \mathscr{F},\left(X_{n}\right)_{n \in \mathbb{N}},\left(\mathbb{P}_{g}\right)_{g} \in G\right)$ la marche aléatoire droite de loi $\mu$ sur $G$; pour tout $g \in G$, nous avons

$$
X_{n}=g Y_{1} \ldots Y_{n}, \quad \mathbb{P}_{g}-\text { p.s. , }
$$

où $\left\{Y_{i}\right\}_{i \geqslant 1}$ est une suite de $v . a$. indépendantes et de loi $\mu$. Désignons par $G=N A K$ (resp. $G_{0}=$ NAK) une décomposition d'Iwasawa de $G$ (voir (1.8)) (resp. de $G_{0}$ ). A est un groupe de Lie abélien simplement connexe; si Cest l'algèbre de Lie de $A$, l'application, $\exp _{A}$, est un isomorphisme analytique du groupe additif de $\boldsymbol{U}$ sur $A$. Ecrivons,

$$
x_{n}=N_{n}\left(\exp H_{n}\right) K_{n} \text { avec } N_{n} \in N, H_{n} \in C \text { et } K_{n} \in K \text {. }
$$

Soient $\Gamma^{\prime}$ l'intersection des normalisateurs dans $G$ de $N$, A et $K$; et $\Gamma$ le centralisateur de $A$ dans $K$. Nous savons (voir $\$ 1$ ) que $\Gamma$ ' rencontre les différentes composantes connexes de $G$, est contenu dans $K$ et son intersection

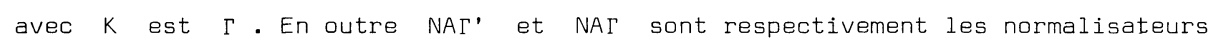
de $N A$ dans $G$ et $G_{0}$.

D'autre part, soit $r=\operatorname{dim} \boldsymbol{U}$. Dans la suite nous désignons par $h_{i}$, $i \in\{1, \ldots, r\}$ un système de fonctions coordonnées par rapport à une certaine base de $\boldsymbol{c}$ et par \|\| une norme sur $\boldsymbol{a}$.

14.2. Comportement de $N_{n}$. - Si $\mu$ est étalée apériodique et possède un moment d'ordre 1 , nous savons (théorème (9.2)) que $N_{n}$ converge, $\mathbb{P}_{x}-$ P.s. $\forall x \in G$, vers une v.a. $N_{\infty}$ à valeurs dans $N$. De plus nous avons :

Lemme. - Pour tout élément $\mathrm{g}$ de $\mathrm{G}$, Za loi $\lambda_{\mathrm{g}}$ de $Z_{a} v . \alpha . \mathrm{N}_{\infty}$, par rapport à la probabilité $\mathbb{P}_{\mathrm{g}}$, est équivalente à la mesure de Haar $\mathrm{m}_{\mathrm{N}}$ de $\mathrm{N}$.

Preỵve. - Soit $Z\left(G_{0}\right)$ le centre de $G_{0} ; Z\left(G_{0}\right)$ est discret et contenu dans $K$. Soit $p$ l'application naturelle de $G$ sur $Z\left(G_{0}\right)^{G}$; quitte à remplacer le couple $(G, \mu)$ par $(p(G), p(\mu))$, nous pouvons supposer ${ }^{\prime}$ que $G_{0}$ est de centre trivial; $K$ est alore compact.

Considérons la décomposition de Bruhat de $G, G=N A \tilde{N}{ }^{\prime} \bmod . m_{G}$; et notons $\beta$ l'application naturelle de $G$ sur $G /{ }^{\prime} \tilde{N}^{\prime}$. La restriction, $\beta_{N}$, de $B$ à $N$ 
est un homéomorphisme de $N$ sur un ouvert de $G /{ }_{A N \Gamma}$, de $B\left(m_{K}\right)$-mesure 1 ( $\left.[26]\right)$. D'après le théorème (9.7) nous avons $\beta\left(\lambda_{g}\right)=\varepsilon_{g} * \beta\left(\lambda_{e}\right)$ et $\beta\left(\lambda_{e}\right)$ est l'unique mesure de probabilité $\mu$-invariante de la frontière maximale droite $G / A \tilde{A} \Gamma$, de $G$. Or la chaine de loi $\mu$ sur ' $/ A N \tilde{}{ }^{\prime}$ ' est ergodique et la probabilité de transition de cette chaîne vérifie la condition de Doeblin pour la mesure $\beta\left(m_{K}\right)$ (voir corollaires (7.2) et (7.6)). D'après [23] nous savons qu'alors $\beta\left(\lambda_{e}\right.$ ) est équivalente à $B\left(m_{K}\right) \cdot B\left(\lambda_{e}\right)$ est donc une mesure quasi-invariante sur ' $/ A \tilde{N} \Gamma$ '. Le lemme s'en déduit immédiatement.

14.3. Comportement de $H_{n}$. - Si $(K, g)$ est un élément de $K \times G$, on désigne par $H(k, g)$ la composante sur $\boldsymbol{a}$ (dans la décomposition $G=N(\exp \boldsymbol{C}) K$ ) de l'élément $\mathrm{kg}$ de $\mathrm{G}$. Il est facile de voir que l'on a

$$
\begin{aligned}
H(\gamma k, g) & =\operatorname{Ad} \gamma(H(k, g)) .\left(\gamma \in \Gamma^{\prime}, k \in K, g \in G\right), \\
\text { (i.e. } \exp H(\gamma k, g) & \left.=\gamma(\exp H(k, g)) \cdot \gamma^{-1}\right) ;
\end{aligned}
$$

en particulier

$$
\begin{gathered}
H(\gamma k, g)=H(k, g) \quad(\gamma \in \Gamma, k \in K, g \in G) \text {. } \\
\text { Considérons alors I'application naturelle } \beta \text { de } G \text { sur } N A \Gamma{ }^{G} \text { et posons } \\
\bar{H}(u, g)=H(k, g) \quad\left(u \in N A \Gamma^{G}, g \in G\right),
\end{gathered}
$$

où $k \in K$ avec $B(k)=u$.

Pour tout couple $(u, g)$ de ${ }_{N A \Gamma} l^{G} \times G, \bar{H}(u, g)$ est bien défini et on vérifie que l'on a

$$
\bar{H}\left(u, g_{1} g_{2}\right)=\bar{H}\left(u, g_{1}\right)+\bar{H}\left(u \cdot g_{1}, g_{2}\right) \quad\left(u \in N A I^{G} ; g_{1}, g_{2} \in G\right) \text {. }
$$

D'où nous avons

$$
H_{n}=\bar{H}\left(B(e), X_{0}\right)+\sum_{i=1}^{n} \bar{H}\left(B\left(X_{i-1}\right), Y_{i}\right) .
$$

Si $\mu$ est apériodique et étalée, nous savons (lemme (7.7)) que la chaîne de

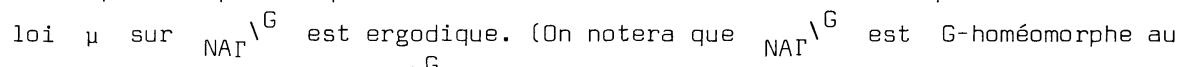
G-espace produit $B(G) \times G_{0} \backslash^{G}$, où $B(G)$ désigne la frontière maximale de $\left.G\right)$. Supposons de plus que

(*)

$$
\int_{N A \Gamma \backslash G} \int_{G}\|\bar{H}(u, g)\|^{2} v(d u) \mu(d g)<+\infty,
$$

où $v$ désigne l'unique mesure $\mu$-invariante de $M^{1}\left({ }_{N A \Gamma}{ }^{G}\right)$. Ce qui est le cas si $\mu$ possède un moment d'ordre 2 . [En effet la fonction

$$
F(g)=\sup _{u \in \in_{N A \Gamma} \backslash^{G}}\|\bar{H}(u, g)\| \quad(g \in G),
$$


est ure fonction sous-additive positive continue de $G$. Si $\mu$ possède un moment d'ordre 2 , nous savons alors (voir (6.3)) que $\int_{G}(F(g))^{2} \mu(d g)<+\infty$ et la condition ( $*$ ) est vérifiée $]$. Alors d'après ([6], ch. 5, \& 7), pour toute probabilité $\mathbb{P}_{g}, g \in G$, le vecteur $H_{n}$ de $C$ est asymptotiquement gaussien, de moyenne $n e_{\mu}$, de matrice de covariance $n \sigma_{\mu}$, où

$$
e_{\mu}=\int_{N A \Gamma} \backslash G \quad \int_{G} \bar{H}(u, g) v(d u) \cdot \mu(d g)
$$

et la matrice de covariance $\sigma_{\mu}$ est non dégénérée (voir [26] prop. 1). Plus précisément, désignons par $\phi_{n}$ la loi gaussienne sur $C$ de paramètres $\left\{n e_{\mu}, n \sigma_{\mu}\right\}$, alors pour tout $g \in G$ et tout $\varepsilon>0$, il existe un entier $n_{0}(\varepsilon)$ tel que pour tout entier $n$ supérieur à $n_{0}$, on ait

$$
\sup _{V}\left|\mathbb{P}_{g}\left[H_{n} \in V\right]-\phi_{n}(V)\right|<\varepsilon,
$$

où $V$ est un borélien de $\boldsymbol{C}$ de la forme $\left\{H \in \mathscr{Q}: h_{i}(H)<a_{i}, i \in\{1, \ldots, r\}\right\}$ pour des réels quelconques $a_{i}, i \in\{1, \ldots, r\}$.

14.4. Comportement de $K_{n}$. - $K_{n}$ n'est autre que la marche de loi $\mu$ sur $K$ identifié à $N A l^{G}$. Si nous supposons que la mesure $\mu$ de $M^{1}(G)$ est étalée, nous savons (voir (10.3)) que cette chaine possède un nombre dénombrable de classes ergodiques, soit $E_{i}, i \in I$, où $I$ est un sous-ensemble fini ou infini de $\mathbb{N}$; et pour tout élément $g$ de $G, K_{n}$ finit par rentrer, $\mathbb{P}_{g}-p . s$. dans une classe ergodique et y reste.

Si $g \in G$, on note $\bar{g}$ sa composante sur $K$ pour la décomposition d'Iwasawa. Comme on a $\overline{\gamma g}=\gamma \bar{g}\left(\gamma \in \Gamma^{\prime}, g \in G\right)$, les éléments de $\Gamma^{\prime}$ permutent les classes

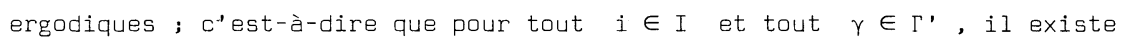
$r(i, \gamma) \in I$ tel que $\gamma E_{i}=E_{r(i, \gamma)}$.

Si de plus $\mu$ est apériodique, on sait que la chaîne de loi $\mu$ sur NAI ${ }^{G}$ est ergodique (lemme (7.7)). On en déduit (voir [26], \$5) que si $A$ et $B$ sont deux classes ergodiques (ou deux sous-classes cycliques) pour la chaine de loi $\mu$ sur $K$, il existe $\gamma \in \Gamma$, tel que $\gamma A=B$. Si $\Gamma$ est connexe, la chaîne de loi $\mu$ sur $K$ est ergodique (voir [26], § 5). Dans le cas général, $\Gamma$ possède un nombre fini de composantes connexes; appelons $s$ le plus petit entier tel que pour tout élément $\gamma$ de $\Gamma$, on ait $\gamma^{5} \in \Gamma_{0}$, composante connexe de l'unité dans $\Gamma$; alors la période d'une quelconque classe ergodique de la chaîne de loi $\mu$ sur $K$ est un diviseur de $s$ (voir $[26], \& 5$ ).

14.5. Indépendance asymptotique. - Soit $\mu$ une mesure de $M^{1}(G)$ étalée, apériodique, possèdant un moment d'ordre 2 . Soit $g$ un élément de $G$ dont la composante, $\bar{g}$, sur $K$ appartient : soit à une sous-classe cyclique $C_{0} d^{\prime} u n e$ classe 
ergodique $E_{i}$ de la chaîne de loi $\mu$ sur $K\left(i_{0} \in I\right)$; soit à une classe ergodique $E_{i_{0}}$ sans classe cyclique de cette chaîne. Dans le premier cas, nous notons $C_{n}$ la soús-classe cyclique de $E_{i}$ contenant, $\mathbb{P}_{g}-p . s ., K_{n}$; nous avons $C_{n+d}=C_{n}$, où $d$ est la période de la classe ergodique $E_{i}$; nous désignons par $\nu_{C} l$ 'unique mesure de $M^{1}\left(C_{n}\right) \mu^{d}$-invariante. Dans le sêcond cas, on convient que $C_{n}=C_{0}=E_{i_{0}} \quad n \in \mathbb{N}$, et $\nu_{C_{n}}=v_{i_{0}}$ est l'unique mesure $\mu$-invariante sur $E_{i_{0}}$.

Avec les notations de (14.2) et (14.3) nous avons alors :

14.6. Théorème : Soit $(G, \mu, g)$ comme en (14.5). Soient $U$ un borélien de $N$ tel que $\mathrm{m}_{N}(\partial U)=0 ; T$ un borélien quelconque de $K$ et

$V=\left\{H \in G: h_{i}(H)<a_{i}, i \in\{1, \ldots, r\}\right\}$, où les $a_{i}$ sont des réels. Alors

nous avons

$$
\lim _{n \rightarrow+\infty} \quad \sup _{V, T}\left|\mathbb{P}_{g}\left\{X_{n} \in U(\exp V) T\right\}-\lambda_{g}(U) \phi_{n}(V) \nu_{C_{N}}(T)\right|=0 .
$$

Preuve. - Nous savons que la composante $N_{n}$ converge $\mathbb{P}_{g}$-p.s. vers une v.a. $N_{\infty}$ dont la loi $\lambda_{g}$ (par rapport à la probabilité $\mathbb{P}_{g}$ ) est équivalente à la mesure de Haar $m_{N}$ (voir (14.2)). Par suite, pour tout borélien $U$ de $N$ tel que $m_{N}(\partial U)=0$, nous avons :

$$
\lim _{n} \mathbb{E}_{g} \quad\left[\left|1_{U}\left(N_{\infty}\right)-1_{U}\left(N_{n}\right)\right|\right]=0 .
$$

Pour tout $\varepsilon>0$, choisissons un entier $n_{1}(\varepsilon)$ tel que $\forall n \geqslant n_{1}(\varepsilon)$ $\mathbb{E}_{g}\left[\left|1_{U}\left(N_{\infty}\right)-1_{U}\left(N_{n}\right)\right|\right]<\varepsilon$

Soit $i \in\{0, \ldots, d-1\}$, pour tout $k \in C_{i},\left\{{\overline{k Y}, \ldots Y_{p d}}_{p \geqslant 1}\right.$ est une chaine ergodique sur $C_{i}$, nous avons donc $([6])$

$$
\left.\underset{k \in C_{i} \quad\{\text { borélieñ }}{\sup } \text { de } C_{i}\right\} \quad\left|\mathbb{P}_{k}\left[\bar{X}_{p d} \in T\right]-v_{C_{i}}(T)\right|<\alpha e^{-\beta p}
$$

où $\alpha$ et $\beta$ sont deux constantes $>0$.

Pour tout $\varepsilon>0$, choisissons un entier $p_{1}(\varepsilon)$ tel que $\alpha e^{-\beta p_{1}(\varepsilon)}<\varepsilon$.

Pour $\varepsilon>0$ donné, écrivons $X_{n}=X_{n_{1}}(\varepsilon) U_{m} T_{P_{1}}(\varepsilon)$ d avec

$n=n_{1}(\varepsilon)+m+p_{1}(\varepsilon) d, U_{m}=Y_{n_{1}+1} \cdots Y_{n_{1}+m}$ et $T_{p_{1} d}=Y_{n_{1}+m+1} \cdots Y_{n_{1}+m+p_{1} d}$.

Posons $U_{m}=N_{m}^{1} A_{m}^{1} K_{m}^{1}$ avec $N_{m}^{1} \in N, A_{m}^{1} \in A, K_{m}^{1} \in K$ et $A_{m}^{1}=\exp H_{m}^{1}$ avec $H_{m}^{1} \in \mathcal{G}$. Nous savons que $N_{m}^{1}$ converge $\mathbb{P}_{g}$-p.s. vers une variable aléatoire $N_{\infty}^{1}$ de $10 i \lambda_{e}$. 
Considérons la décomposition de Bruhat $G=N \tilde{N N}^{\prime}$. Comme $x_{n_{1}}$ et $U_{m}$ sont des v.a. indépendantes pour tout $m \in \mathbb{N}$, nous avons

et $\mathbb{P}_{g}\left[x_{n_{1}} N_{m}^{1} \notin N A \tilde{N}^{\prime}\right]=\int \varepsilon_{g} * \mu^{n}(d x) \mathbb{P}_{e}\left[x N_{m} \notin N A \tilde{N} \Gamma^{\prime}\right]$

$$
\mathbb{P}_{g}\left[x_{n_{1}} N_{\infty}^{1} \notin N A \tilde{N} \Gamma^{\prime} \mid=\int \varepsilon_{g} * \mu^{n_{1}}(d x) \mathbb{P}_{e}\left[x N_{\infty} \notin N \tilde{N}^{\prime} \Gamma^{\prime}\right] .\right.
$$

D'après le lemme (9.11) et (9.13) il s'ensuit que pour $\mathbb{P}_{g}$-presque tout $\omega$ de $\Omega$, il existe $m_{0}(\omega)$ tel que

i) $\forall m \geqslant m_{0}(\omega), \quad x_{n_{1}} N_{m}^{1} \in N A \tilde{N} \Gamma^{\prime}$

ii) $\lim _{n} x_{n_{1}}(\omega) N_{m}^{1}(\omega)=x_{n_{1}}(\omega) N_{\infty}^{1}(\omega) \in N A N^{\prime} \Gamma^{\prime}$.

On peut alors trouver un compact $Q_{\varepsilon}^{1}$ de I'ouvert $N A \tilde{N}^{\prime}$ ' de $G$ et un entier $m_{1}(\varepsilon)$ tel que

$P_{g}\left[\left\{x_{n_{1}} N_{m}^{1} \in Q_{\varepsilon}^{1}, \forall m \geqslant m_{1}\right\} \cap\left\{\lim x_{n_{1}} N_{m}^{1}=X_{n_{1}} N_{\infty}^{1} \in Q_{\varepsilon}^{1}\right\}\right]>1-\varepsilon / 2$.

D'autre part, choisissons un compact $Q_{\varepsilon}^{2}$ de $G$ (que nous pouvons supposer vérifier $Q_{\varepsilon}^{2}=\underset{k \in K}{U} k Q_{\varepsilon}^{2} k^{-1}$ ) tel que $\mathbb{P}_{g}^{\varepsilon}\left[T_{P_{1} d} \in Q_{\varepsilon}^{2}\right]>1-\varepsilon / 2$. Posons

$\Omega_{\varepsilon}=\left\{X_{n_{1}} N_{m}^{1} \in Q_{\varepsilon}^{1}, \forall m \geqslant m_{1}\right\} \cap\left\{\underset{n}{\lim } X_{n_{1}} N_{m}^{1}=X_{n_{1}} N_{\infty}^{1} \in Q_{\varepsilon}^{1}\right\} \cap\left\{T_{p_{1} d} \in Q_{\varepsilon}^{2}\right\} ;$

nous avons $\mathbb{P}_{g}\left(\Omega_{\varepsilon}\right)>(1-\varepsilon / 2)^{2}>1-\varepsilon$ (car $T_{P_{1} d}$ est indépendant de $x_{n_{1}}$ et de $\left.U_{m}\right\}$.

Sur $\Omega_{\varepsilon}$ nous avons, $x_{n_{1}} N_{m}^{1}=u_{m} a_{m} \gamma_{m} \tilde{u}_{m}$, où $u_{m}, a_{m}, \gamma_{m}$ et $\tilde{u}_{m}$ sont des suites qui convergent respectivement vers des éléments $u, a, \gamma$ et $\tilde{u}$ en restant dans un compact fixé respectivement de $N, A, \Gamma$ ' et $\tilde{N}$. D'où

$$
x_{n}=u_{m} a_{m}\left(\gamma_{m} A_{m}^{1} \gamma_{m}^{-1}\right) \gamma_{m}\left[\left(A_{m}^{1}\right)^{-1} \tilde{u}_{m} A_{m}^{1}\right] K_{m}^{1} T_{p_{1} d} \cdot
$$

Désignons par $s$ une section de l'application naturelle $\zeta$ de $G$ sur $G_{0}{ }^{G}$, à valeurs dans $\Gamma^{\prime}$. Comme $\Gamma$ centralise $A$, nous avons sur $\Omega_{\varepsilon}$

c'est-à-dire

$$
\begin{aligned}
\gamma_{m} A_{m}^{1} \gamma_{m}^{-1} & =s \circ \zeta\left(X_{n_{1}} N_{m}^{1}\right) A_{m}^{1}\left(s \circ \zeta\left(X_{n_{1}} N_{m}^{1}\right)\right)^{-1} \\
& =s \circ \zeta\left(x_{n_{1}}\right) A_{m}^{1}\left(s \circ \zeta\left(x_{n_{1}}\right)\right)^{-1} ;
\end{aligned}
$$

$$
\text { Ad } \gamma_{m}\left(H_{m}^{1}\right)=\operatorname{Ad} s \circ \zeta\left(x_{n_{1}}\right)\left(H_{m}^{1}\right)=H\left(s \circ \zeta\left(x_{n_{1}}\right), U_{m}\right) .
$$

D'autre part, nous savons (lemme (9.9) que $\left(A_{m}^{1}\right)^{-1} \times A_{m}^{1}, x \in \tilde{N}$, converge, $\mathbb{P}_{\text {g }}$-p.s., vers $e$, uniformément sur tout compact de $\tilde{N}$. Comme on a 
$H_{n}=\exp _{A}^{-1} a_{m}+H\left(s \circ \zeta\left(x_{n_{1}}\right), U_{m}\right)+H\left(e, \gamma_{m}\left(\left(A_{m}^{1}\right)^{-1} \tilde{u}_{m} A_{m}^{1}\right) K_{m}^{1} T_{p_{1} d}\right)$,

on en déduit qu'il existe un sous-ensemble de $\Omega_{\varepsilon}$ de même $\mathbb{P}_{\mathrm{g}}$-mesure et une constante $\gamma\left(Q_{\varepsilon}^{1}, Q_{\varepsilon}^{2}\right)$ tels que sur ce sous-ensemble, que nous noterons toujours $\Omega_{\varepsilon}$, on ait, pour $m \geqslant m_{1}$,

$$
\left\|H_{m}-H\left(s \circ \zeta\left(x_{n_{1}}\right), U_{m}\right)\right\|<\gamma
$$

D'après (14.3), pour tout $\varepsilon>0$, il existe un entier $m_{2}(\varepsilon)$ tel que $\forall m_{2} \geqslant m_{2}$ $\sup _{V} \sup _{y \in G_{0} \backslash^{G}}\left|\mathbb{P}_{g}\left(H\left(s(y), U_{m}\right) \in V\right)-\phi_{m}(V)\right|<\varepsilon / 2 \quad$ (noter que $G_{0} \backslash^{G}$ est fini),

et

$$
\sup _{V}\left|\mathbb{P}_{g}\left(H\left(s\left(X_{n_{1}}\right), U_{m}\right) \in V\right)-\phi_{m}(V)\right|<\varepsilon / 2,
$$

$$
\sup _{V}\left|\mathbb{P}_{g}\left[H_{n} \in V\right]-\phi_{n}(V)\right|<\varepsilon
$$

D'autre part, comme la matrice de covariance $\sigma_{\mu}$ est non dégénérée, il existe un entier $n_{2}(\varepsilon)$ tel que pour tout $n \geqslant n_{2}$ $\sup _{V} \quad i \in\{1, \ldots, r\} \quad \operatorname{Pup}_{g}\left[a_{i}-\gamma \leqslant h_{i}\left(H_{n}\right) \leqslant a_{i}+\gamma\right]<\varepsilon / r$.

Ceci dit, soit $\varepsilon>0$ donné. Choisissons

$$
n>\sup \left\{n_{2}(\varepsilon), n_{1}(\varepsilon)+\sup \left\{m_{1}(\varepsilon), m_{2}(\varepsilon)\right\}+p_{1}(\varepsilon) d\right\}
$$

et posons $n=n_{1}(\varepsilon)+m+p_{1}(\varepsilon) d$. Nous avons :

$$
\begin{aligned}
& \text { a) } \sup _{V, T}\left|\mathbb{P}_{g}\left[N_{n} \in U, H_{n} \in V, K_{n} \in T\right]-\mathbb{P}_{g}\left[N_{n_{1}} \in U, H_{n} \in V, K_{n} \in T\right]\right| \leqslant \\
& \mathbb{E}_{g}\left[\left|1_{U}\left(N_{n}\right)-1_{U}\left(N_{n_{1}}\right)\right|\right]<2 \varepsilon,
\end{aligned}
$$

d'après le choix de $n_{1}(\varepsilon)$.

b) $\left(\left\{H_{n} \in V\right\} \Delta\left\{H\left(s \circ \zeta\left(x_{n_{1}}\right), U_{m}\right) \in V\right\}\right) \cap \Omega_{\varepsilon} \subset i \in\{\underset{1}{\cup} \ldots, r\}\left\{a_{i}-\gamma \leqslant h_{i}\left(H_{n}\right)\right.$

puisque $m>m_{1}$. Par suite

$$
\left.\leqslant a_{i}+\gamma\right\} \text {, }
$$

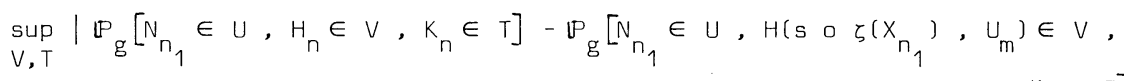

$$
\begin{aligned}
& \left.k_{n} \in T\right] \mid \\
& \leqslant \sup _{V} \mathbb{P}_{g}\left[\left\{H_{n} \in V\right\} \Delta\left\{H\left(s \quad 0 \quad \zeta\left(X_{n_{1}}\right), U_{m}\right) \in V\right\}\right] \\
& <\varepsilon+r \underset{i \in\{1, \ldots, r\}}{\sup } \quad \sup _{V} \mathbb{P}_{g}\left[\left\{a_{i}-\gamma \leqslant h_{i}\left(H_{n}\right) \leqslant a_{i}+\gamma\right\}\right] \\
& <2 \varepsilon \text {, puisque } \Pi>\Pi_{2}(\varepsilon) \text {. }
\end{aligned}
$$




$$
\begin{aligned}
& \text { c) } \sup _{V, T} \mid \mathbb{P}_{g}\left[\mathrm{~N}_{\mathrm{n}_{1}} \in U, H\left(s \circ \zeta\left(\mathrm{x}_{\mathrm{n}_{1}}\right), \mathrm{U}_{\mathrm{m}}\right) \in V, \mathrm{~K}_{\mathrm{n}} \in T\right]-\mathbb{P}_{\mathrm{g}}\left[\mathrm{N}_{\mathrm{n}_{1}} \in U\right. \text {, } \\
& \left.H\left(s \circ \zeta\left(X_{n_{1}}\right), U_{m}\right) \in V\right] v_{C_{n}}(T)\left|=\sup _{V, T}\right| \mathbb{E}_{g}\left[1_{U}\left(N_{n_{1}}\right) 1_{V}\left(H\left(s \circ \zeta\left(X_{n_{1}}\right), U_{m}\right)\right)\right. \\
& \left.\left.\mathbb{E}_{g}\left[1_{T}\left(K_{n}\right) \mid \mathcal{F}_{n_{1}+m}\right]-v_{C_{n}}(T)\right)\right] \mid \text {, } \\
& \text { où } \mathcal{F}_{n_{1}+m} \text { désigne la tribu engendrée par les v.a. } x_{0}, \ldots, x_{n_{1}+m} \\
& \left.\leqslant \sup _{T} \mathbb{E}_{g}\left[\left|\mathbb{E}_{g}\right| 1_{T}\left(K_{n}\right) \mid \mathcal{F}_{n_{1}+m}\right]-v_{C_{n_{1}+m}}(T) \mid\right] \text { (noter que } C_{n_{1}+m}=C_{n} \text { ) } \\
& \leqslant \sup _{T} \mathbb{E}_{g}\left[\left|\mathbb{E}_{X_{n_{1}+m}}\right| 1_{T}\left(K_{P_{1} d}\right)\left|-v_{C_{n_{1}+m}}(T)\right|\right] \quad \text { (propriété de Markov) } \\
& \leqslant \sup _{k \in C_{n_{1}+m}} \sup _{T}\left|\mathbb{P}_{k}\left(\bar{X}_{p_{1} d} \in T\right)-v_{C_{n_{1}+m}}(T)\right| \\
& \leqslant \sup _{i \in\{0, \ldots, d-1\}} \sup _{k \in C_{i}} \sup _{T}\left|\mathbb{P}_{k}\left(\bar{X}_{p_{1} d} \in T\right)-\nu_{C_{i}}(T)\right| \\
& <\varepsilon ; \text { d'après le choix de } P_{1}(\varepsilon) \text {. } \\
& \text { d) } \sup _{V}\left|\mathbb{P}_{g}\left[N_{n_{1}} \in U, H\left(s \circ \zeta\left(x_{n_{1}}\right), u_{m}\right) \in V\right]-\mathbb{P}_{g}\left[N_{n_{1}} \in U\right] \mathbb{P}_{g}\left[H_{n} \in V\right]\right| \\
& =\sup _{V} \mid \int_{\Omega} 1\left(N_{n_{1}}(\omega)\right)\left[\mathbb{P}_{g}\left[H\left(s \circ \zeta\left(x_{n_{1}}(\omega)\right), U_{m}\right) \in V\right]-\mathbb{P}_{g}\left[H_{n} \in V\right]\right] d \mathbb{P}_{g}(\omega) \\
& \text { car } X_{n_{1}} \text { et } U_{m} \text { sont des } v \cdot a \text {. indépendantes }
\end{aligned}
$$

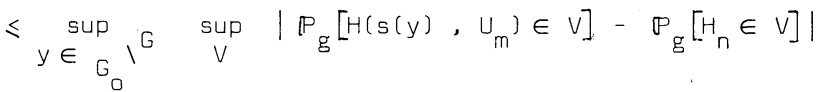

$$
\begin{aligned}
& <\varepsilon / 2+\varepsilon / 2+\sup _{V}\left|\mathbb{P}_{g}\left(H\left(s \circ \zeta\left(X_{n_{1}}\right), U_{m}\right) \in V\right)-\mathbb{P}_{g}\left(H_{n} \in V\right)\right| \text { (puisque } m>m_{2} \text { ) } \\
& <\varepsilon+\sup _{V} \mathbb{P}_{g}\left[\left\{H_{n} \in V\right\} \Delta\left\{H\left(s \circ \zeta\left(x_{1}\right), U_{m}\right) \in V\right\}\right] \\
& <3 \varepsilon \text { (d'après b) ). } \\
& \text { e) }\left|\mathbb{P}_{g}\left[N_{n_{1}} \in U\right]-\lambda_{g}(U)\right|<\mathbb{E}_{g}\left[\left|1_{U}\left(N_{n_{1}}\right)-1_{U}(Z)\right|\right]<\varepsilon \text {, } \\
& \text { d'après le choix de } n_{1}(\varepsilon) \text {. Et } \\
& \underset{V}{\operatorname{suf}}\left|\mathbb{P}_{g}\left[H_{n} \in V\right]-\phi_{n}(V)\right|<\varepsilon \quad \text { car } m>m_{2}(\varepsilon) .
\end{aligned}
$$


De a), b), c), d) et e) il résulte alors que l'on a,

$\sup _{V, T}\left|\mathbb{P}_{g}\left[N_{n} \in U, H_{n} \in V, K_{n} \in T\right]-\lambda_{g}(U) \phi_{n}(V) \nu_{C_{n}}(T)\right|<10 \varepsilon$.

Le théorème (14.6) est prouvé.

14.7. Remarque. - Dans ce qui précède, on s'est donné une décomposition d'Iwasawa et on a étudié le comportement asymptotique, dans cette représentation, de la loi de la marche aléatoire droite de loi $\mu$ sur $G$. Supposons que pour une mesure donnée, on s'intéresse seulement au comportement de $\mu^{n}$. On peut alors trouver une décomposition d'Iwasawa NAK de $G$ telle que l'élément neutre e de G appartienne : soit à une sous-classe cyclique d'une classe ergodique de la chaîne de loi $\mu$ sur $K$; soit à une classe ergodique sans classe cyclique de cette chaîne. En fait, si $N A K$ est une quelconque décomposition d'Iwasawa de $G$, la décomposition d'Iwasawa, $\left(\mathrm{gNg}^{-1}\right)\left(\mathrm{gAg}^{-1}\right)\left(\mathrm{gK}^{-1}\right)$, vérifie cette propriété pour $m_{G}$-presque tout élément de $g$ de $G$. Pour une telle décomposition d'Iwasawa, nous avons alors, avec la notation du théorème (14.6),

$$
\lim _{n} \sup _{V, T}\left|\mu^{n}(U(\exp V) T)-\lambda_{g}(U) \phi_{n}(V) \nu_{C_{n}}(T)\right|=0 .
$$

14.8. Théorème. ' - Soient $(G, \mu)$ comme en (14.5) et $\left(\Omega, \mathcal{F},\left(X_{n}\right)_{n \in \mathbb{N}},\left(\mathbb{P}_{x}{ }^{j} \times \in G\right)\right.$ Za marche aléatoire droite de loi $\mu$ sur $G$. Considérons les décompositions $G=N A K \quad($ Iwasawa $)$ et $G=B_{0}(\exp W) K \bmod m_{G}(\operatorname{voir}(14.0)) . \times$ désignant un élément quelconque de $\mathrm{G}$, pour $\mathbb{P}_{\mathrm{x}}$-presque tout $\omega$ de $\Omega, \mathrm{x}_{\mathrm{n}}(\omega)$ s'écrit, $\grave{a}$ partir d'un certain rang,

$$
x_{n}(\omega)\left(\exp h_{n}(\omega)\right) k_{n}(\omega),
$$

où $\left\{h_{n}(\omega)\right\}$ et $\left\{k_{n}(\omega)\right\}$ sont des suites d'éléments respectivement de $w$ et $k$, $\left\{x_{n}(\omega)\right\}$ est une suite d'éléments de $\mathrm{B}_{0}$ convergeant vers un élément $x_{\infty}(\omega)$ de $\mathrm{B}_{0}$. De plus si $\nu_{x}$ désignie la loi de $x_{\infty}$ par rapport à $\mathbb{P}_{x}$, l'image de $\nu_{x}$ par l'application naturelle de $G$ sur $G /{ }_{A N \Gamma}$ ' s'écrit $\varepsilon_{x} * \nu$, où $\nu$ désigne l'unique mesure u-invariante de $M^{1}\left({ }^{G} /{ }^{A N} \Gamma^{\prime}\right)$.

$D^{\prime a u t r e}$ part, soiént $U$ et $T$ des boréliens respectivement de $\mathrm{B}_{0}$ et $K$ tels que $m_{K}(\Gamma(\partial U))=0$ et $m_{K}(\partial T)=0$. Alors si $g$ est un élément de $G$ comme en (14.5) nous avons

$$
\lim _{n} \sup _{V}\left|\mathbb{P}_{g}\left[x_{n} \in U(\exp V) T\right]-\nu_{g}(U) \phi_{n}(V) \nu_{C_{n}}(T)\right|=0,
$$

où $\phi_{n}, v_{c_{n}}$ et $V$ sont comme dans le théorème (14.6). 
Preuve : Reprenons les notations des paragraphes 1 et 2 : désignons par $y=\mathcal{N} \oplus Q \oplus K$ la décomposition d'Iwasawa de l'algèbre de Lie ef qui correspond à celle $G_{0}=N A K$ de $G_{0}$; par $\Sigma_{1}$ l'ensemble des poids de la représentation adjointe de $\mathcal{Q}$ dans $\mathcal{N}$. Pour tout $\lambda \in \Sigma_{1}$, on note $\phi_{\lambda}$ le poids de la représentation adjointe de $A$ dans $\mathcal{N}$ dont la différentielle en e est $\lambda$. Nous avons alors :

14.9. Lemme. - (D'après Virtser, [26] lemme 4). Soient $\left\{a_{n}\right\}$ une suite d'éléments de A telle que:

i) $\log .\left|\phi_{\lambda}\left(a_{n}\right)\right| \rightarrow-\infty \quad \forall \lambda \in \Sigma_{1}$

ii) $\sup _{n} \frac{\log \left|\phi_{\lambda}\left(a_{n}\right)\right|}{\log \left|\phi_{\lambda},\left(a_{n}\right)\right|}<+\infty \quad \forall \lambda, \lambda^{\prime} \in \Sigma_{1}$;

et $C$ un compact de $\tilde{N}$ tel que $\tilde{u} a_{n}$ appartienne à $K(\exp W) K$ pour tout entier naturez $n$ et tout élément $\tilde{u}$ de $c$.

Alors à partir d'un certain rang, nous pouvons écrire

$$
\tilde{u} a_{n}=x_{n}(\tilde{u}) a_{n}^{\prime}(\tilde{u}) k_{n}(\tilde{u}) \quad(\tilde{u} \in C),
$$

où $\left\{x_{n}(\tilde{u})\right\}$ (resp. $\left.\left\{k_{n}(\tilde{u})\right\}\right)$ est une suite d'éléments de $\mathrm{B}_{0}$ (resp. $\mathrm{K}$ ) convergeant, uniformément sur $c$, vers $e$, et $\left\{a_{n}^{\prime}(\tilde{u})\right\}$ est une suite d'éléments de $\exp W$ telze que $\left\{a_{n}^{-1}(\tilde{u}) a_{n}^{\prime}(\tilde{u})\right\}$ converge, uniformément sur $c$, vers $e$.

Preuve. - Pour $\tilde{u} \in c$, écrivons $\tilde{u} a_{n}=x_{n}^{\prime} a_{n}^{\prime} k_{n}^{\prime}$ avec $x_{n}^{\prime} \in k, a_{n}^{\prime} \in \exp w$ et $k_{n}^{\prime} \in K$. Posons $B=K \cap N A \tilde{N} \Gamma$; $B$ est un ouvert partout dense de $K$ (voir [26]). Désignons par $B$ l'application naturelle de $K$ sur $K / \Gamma, B\left(B_{0}\right)=\beta(B)$ est alors un ouvert partout dense de $\mathrm{K} / \Gamma_{\Gamma}$.

D'après la preuve du lemme 4 de [26], nous savons que la suite $\left\{x_{n}^{\prime}\right\}$ a ses valeurs d'adhérence qui appartiennent nécessairement à $\Gamma$. Autrement dit la suite $\left\{\beta\left(x_{n}^{\prime}\right)\right\}$ de $K / \Gamma$ converge vers $\beta(e)$. A partir d'un certain rang nous avons donc $x_{n}^{\prime} \in B$ et $x_{n}^{\prime}$ s'écrit $t_{n} \gamma_{n}$ avec $t_{n} \in B_{0}$ et $\gamma_{n} \in \Gamma$. Nous avons alors $\tilde{u} a_{n}=t_{n} a_{n}^{\prime}\left(\gamma_{n} k_{n}\right)$ et nous pouvons appliquer les. conclusions du lemme 4 de [26]. Le lemme (14.9) est établi.

Preuve du théorème 14.8. - Ecrivons $x_{n}=N_{n}\left(\exp H_{n}\right) K_{n}$ avec $N_{n} \in N, H_{n} \in C$ et $K_{n} \in K$. . Considérons la décomposition d'Iwasawa, $G_{0}=K A \tilde{N}$, de $G_{0}$ et posons $N_{n}=y_{n} \tilde{u}_{n} \alpha_{n}$ avec $y_{n} \in K, u_{n} \in \tilde{N}$ et $\alpha_{n} \in A$. Remarquons que $y_{n}=N_{n} \alpha_{n}^{-1} \tilde{u}_{n}^{-1}$ appartient nécessairement à $B_{0}$. Soit $x \in G$. Comme $N_{n}$ converge, $\mathbb{P}_{x}$-p.s., vers $N_{\infty}$, les suites $y_{n}$, $\tilde{u}_{n}$ et $\alpha_{n}$ convergent $\mathbb{P}_{x}-p . s$. res- 
pectivement vers des variables aléatoires $y, \tilde{U}$ et $\alpha$, à valeurs respectivement dans $B_{0}, \tilde{N}$ et $A$ telles que $N_{\infty}=y \tilde{u} \alpha$.

$\mu$ étant étalée, il est facile de voir (cf. (9.13)) que l'on a

$\sum_{n \in N} \mathbb{P}_{X}\left[X_{n} \in B_{0}(\exp W) K\right]<+\infty$. Il existe alors un sous ensemble mesurable $\Omega^{\prime}$ de $\mathbb{P}_{x}$-mesure 1 tel que pour tout $\omega \in \Omega^{\prime}$, il existe un entier $n_{0}(\omega)$ pour lequel $\forall n \geqslant n_{0}(\omega)$.

$x_{n}(\omega)=x_{n}(\omega) \exp h_{n}(\omega) k_{n}(\omega)$ avec $x_{n} \in B_{0}, h_{n} \in w$ et $k_{n} \in K$.

Sur $\Omega^{\prime}$ nous avons alors à partir d'un certain rang

$$
\tilde{u}_{n}\left(\alpha_{n} \exp H_{n}\right)=\left(y_{n}^{-1} x_{n}\right)\left(\exp h_{n}\right)\left(k_{n} k_{n}^{-1}\right) \text {. }
$$

Désignons par $\left\{\tau_{\lambda}, \lambda \in \Sigma_{1}\right\}$ la famille d' $A$-cocycles sur $B(G) \times{ }_{G} \backslash^{G}$ associée à $G$ pour la décomposition d'Iwasawa $G=N A K$ et par $v_{0}$ l'unique élément de $B(G) \times G_{0} \backslash^{G}$ invariant par NAT (voir $\left.\& 2\right)$. Posons $\Omega^{\prime \prime}=\left\{\omega \in \Omega^{\prime}: \lim N_{n}(\omega)=N_{\infty}(\omega)\right.$ $\left.\frac{1}{n} \tau_{\lambda}\left(v_{0}, x_{n}(\omega)\right) \rightarrow a_{\lambda}<0, \forall \lambda \in \Sigma_{1}\right\}, \Omega$ est de $\mathbb{P}_{x}$-mesure 1 (voir (9.5)). Pour tout $\omega \in \Omega$ " la suite $\alpha_{n}(\omega) \exp H_{n}(\omega)$ vérifie les hypothèses i) et ii) du lemme (14.9). En effet pour tout $\lambda \in \Sigma_{1}$, nous avons sur $\Omega$ ",

$$
\begin{aligned}
\frac{1}{n} \log \left|\phi_{\lambda}\left(\alpha_{n} \exp H_{n}\right)\right| & =\frac{1}{n} \log \left|\phi_{\lambda}\left(\alpha_{n}\right)\right|+\frac{1}{n} \log \left|\phi_{\lambda}\left(\exp H_{n}\right)\right| \\
& =\frac{1}{n} \log \left|\phi_{\lambda}\left(\alpha_{n}\right)\right|+\frac{1}{n} \tau_{\lambda}\left(v_{0}, x_{n}\right) \quad(\operatorname{voir}(2.9)) \\
& \longrightarrow a_{\lambda}<0 .
\end{aligned}
$$

D'après le lemme (14.9), sur $\Omega$ ". , nous avons donc à partir d'un certain rang

$$
\tilde{u}_{n}\left(\alpha_{n} \exp H_{n}\right)=t_{n} \exp \left(h_{n}\right) k_{n}^{\prime} \text {, }
$$

où $\left\{t_{n}\right\}$ (resp $\left\{k_{n}^{\prime}\right\}$ ) est une suite d'éléments de $B_{0}$ (resp. de $K$ ) convergeant vers $e,\left\{h_{n}\right\}$ est une suite d'éléments de $W$ telle que $\left(\alpha_{n} \exp H_{n}\right) \exp \left(-h_{n}\right)$ converge vers e.

On en déduit que sur $\Omega$ ", nous avons à partir d'un certain rang

$$
x_{n}=y_{n} t_{n} \gamma_{n} \text { avec } \quad \gamma_{n} \in \Gamma
$$

Mais comme $x_{n} \in B_{0}$ et $y_{n} t_{n}$ converge vers $y \in B_{0}$, les suites $\left\{x_{n}\right\}$ et $\left\{\gamma_{n}\right\}$ convergent respectivement vers $y$ et $e$.

Soient $\nu_{x}\left(r e s p \quad \lambda_{x}\right.$ ) la loi de la v.a. y (resp. $N_{\infty}$ ) par rapport à $\mathbb{P}_{x}$. Comme les mesures images de $\nu_{x}$ et $\lambda_{x}$ par l'application naturelle de $G$ sur G/ $\tilde{A N T}^{\prime}$, sont identiques, la première assertion du théorème (14.8) est prouvée.

Soient $U$ et $T$ des boréliens respectivement de $B_{0}$ et $K$ tels que $m_{K}(\Gamma(\partial U))=0$ et $m_{K}(\partial T)=0$. 
Nous savons que sur $\Omega^{\prime}$, à partir d'un certain rang, on a :

i) $x_{n}=y_{n} t_{n}^{\prime}$, où $t_{n}^{\prime}$ converge vers e et $y_{n}$ converge vers une $v . a$. $y$ dont la loi $\nu_{x}$, par rapport à $\mathbb{P}_{x}$, vérifie $\nu_{x}(\partial U)=0$.

(Car $m_{K}(\Gamma(\partial U))=0$ et $I$ 'image de $\nu_{x}$ par l'application naturelle de $K$ sur

$K / \Gamma$ est la restriction à un ouvert d'une mesure quasi-invariante de $K / \Gamma$ ).

ii) $k_{n}=k_{n}^{\prime \prime} k_{n}$, où $k_{n}^{\prime \prime}$ converge vers e et les mesures $v_{C_{n}}$ sont absolument continues par rapport à la mesure de Haar de $K$.

Il est clair alors que l'on a

$$
\begin{aligned}
& \lim \sup _{n}\left|\mathbb{P}_{x}\left[x_{n} \in U, h_{n} \in V, k_{n} \in T\right]-\mathbb{P}_{x}\left[y_{n} \in U, h_{n} \in V, k_{n} \in T\right]\right| \\
& \leqslant \lim _{n} \mathbb{P}_{x}\left[\left(x_{n} \in U, k_{n} \in T\right) \Delta\left(y_{n} \in U, k_{n} \in T\right)\right]=0 .
\end{aligned}
$$

D'autre part sur $\Omega$ ", $H_{n}{ }^{-h_{n}}$ converge vers $\exp _{A}^{-1} \alpha$. Vu le comportement de $H_{n}$ (voir (14.3)), il est alors clair que l'on a

$$
\lim \sup _{\mathrm{n}}\left|\mathbb{P}_{x}\left[\left(H_{n} \in V\right) \Delta\left(h_{n} \in V\right)\right]\right|=0 \text {. }
$$

On en déduit que l'on a

$$
\begin{aligned}
& \lim \sup _{V}\left|\mathbb{P}_{x}\left[x_{n} \in U, h_{n} \in V, k_{n} \in T\right]-\mathbb{P}_{x}\left[y_{n} \in U, H_{n} \in V, K_{n} \in T\right]\right|=0 . \\
& \text { Désignons par } \beta \quad l \text { 'application naturelle de }{ }_{0} /{ }_{A N \Gamma}(\approx K / \Gamma) \text {. La restriction } \beta_{N}
\end{aligned}
$$
(resp. $\beta_{B_{0}}$ ) de $\beta$ à $N$ (resp. à $B_{0}$ ) est un homéomorphisme de $N$ (resp. $B_{0}$ ) sur son image qui est un ouvert partout dense de $\% /{ }^{2} \tilde{N}$. En notant alors que $\mathbb{P}_{x}\left[y_{n} \in U, H_{n} \in V, K_{n} \in T\right]=\mathbb{P}_{x}\left[N_{n} \in \beta_{n}^{-1} \circ \beta_{B_{0}}(U), H_{n} \in V, K_{n} \in T\right]$, la deuxième assertion du théorème résulte du théorème (14.6).

\section{REFERENCES}

[1] AZENCOTT R., Espaces de Poisson des groupes Zocalement compacts. Lecture notes, Springer-Verlag $n^{\circ} 148$, (1970).

[2] AZENCOTT R. et CARTIER P., Martin boundaries of random walks on Zocalzy compact groups. 6th Berkeley Symposium Proc. Math. Statist. Probab. 3, Univ. Calif. Press. (1972), p. 87-123.

[3] BIRGe L. et RAUGi A., Comptes Rendus 278, série A, (1974), 1287.

[4] BRंOWN I.D. et GUIVARC'H Y., Espace de Poisson des groupes de Lie. Extrait de Ann. Scient. de l'E.N.S., 4è série, t.7, fasc. 2, (1974). 
[5] CHeVAlLey C., Théorie des groupes de Lie. Publ. de l'Inst. de Math. de l'Université de Nancago, I et IV.

[6] DOOB J.L., Stochastic Processes. John Wiley, New York, (1953).

[7] ELIE L. et RAUGI A., Comptes Rendus, 280, série A, (1975), P. 377.

[8] FURSTENBERG H., A Poisson formula for semi-simple Lie groups. Ann. of Math., 77, (1963), 335-386.

[9] FURSTENBERG H., Amer. Math. Soc. Summer Inst. on Harmonic Analysis on Homogeneous spaces, Williamstown, Massachussets, (1972).

[10] FURSTENBERG H., Non commuting random products. Trans. Amer. Math. Soc. Vol. 108, $(1963), 377-428$.

[11] GUIVARC'H Y., Croissance polynomiale et périodes des fonctions harmoniques. Bull. Soc. Math. France, 101, (1973), 333-379.

[12] GUIVARC'H Y., Lois des grands nombres sur les groupes. A paraitre.

[13] HELGASON S., Differential geometry and symetric spaces. New York, Acad. Press, (1962).

[14] HOCHSCHILD G., The structure of Lie groups. Holden Day Inc, San Francisco, USA (1965).

[15] IWASAWA K., Ann. of Math., 50, $n^{\circ} 3$, (1949).

[16] JACOD J., Théorèmes de renouvellement et classification pour les chaînes semi-markoviennes. Ann. Inst. H. Poincaré, Vol. 7, $n^{\circ} 2,(1971), 83-129$.

[17] KELLEY J., General Topology. New York, D-Van Nostrand Company, Inc. (1955)

[18] KESTEN, The limit points of a normalised random walk. Ann. Math. Statist. 41, (1970), 1173-1205.

[19] MACKEY G., Induced representations of Zocally compact groups. Ann. of Math. $55(1952), 101-139$.

[20] MONTGOMERY D. and ZIPPIN L., Topological Transformation groups. New York, Interscience publisher, (1955).

[21] RAUGi A., Comptes Rendus, 280, série A, (1975), 1309.

[22] RAUGi A., Comptes Rendus, 282, série A, (1976), 653.

[23] REVUZ D., Markov chains. North-Holland Mathematical Library, Vol. 11, (1975).

[24] ROSENBERG J., Remarks on random walks on semi-simple Lie groups. A paraître.

[25] Séminaire Sophus Lie, (1954-1955), Paris, exposé 9 (F. Bruhat).

[26] VIRTSER A.D., Central limit theorem for semi-simple Lie groups. Theory of probability and its applications, (1970, Vol. XV, 667-687.

Albert RAUGI

Mathématiques, Université de Rennes

35042 RENNES CEDEX 